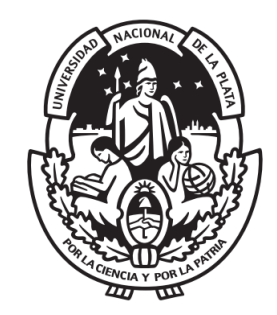

Universidad Nacional de La Plata

FACUlTad DE Ciencias EXactas

DePARTAMENTO DE MATEMÁTICA

TESIS PRESENTADA PARA OPTAR AL GRADO DE DOCTOR DE LA FACULTAD DE Ciencias Exactas

\title{
El método LCB y la estabilización de sistemas subactuados
}

\author{
Autor: Mgter. Leandro Martín Salomone
}

Director: Dr. Sergio Daniel Grillo

Codirectora: Dra. Marcela Zuccalli 



\section{Índice general}

Gracias $\quad$ IX

Introducción y organización de la tesis $\quad \mathbf{X V}$

1. Conceptos preliminares 1

1.1. Notación . . . . . . . . . . . . . . . . . . . . . . . 1

1.2. Conexiones afines sobre un fibrado vectorial y el isomorfismo $\beta \ldots \ldots \ldots$

1.3. Sistemas dinámicos y estabilización tipo Lyapunov $\ldots \ldots \ldots$. . . . . . . . . 9

1.3.1. Estabilidad asintótica y el Principio de invariancia de LaSalle . . . . . . . . . . 11

1.3.2. Sistemas Hamiltonianos . . . . . . . . . . . . . . . . . . . 12

1.4. El método de estabilización basado en el vínculo de Lyapunov . . . . . . . . . . . 13

1.4.1. Sistemas a lazo cerrado y sistemas con vínculos de segundo orden . . . . . . . 13

1.4.2. El Vínculo de Lyapunov y el método LCB . . . . . . . . . . . . . . . . . 15

2. Equivalencia de métodos de estabilización y maximalidad del método LCB 21

2.1. Métodos de estabilización y equivalencia de métodos . . . . . . . . . . . . 23

2.2. El método energy shaping . . . . . . . . . . . . . . . . . . . . . . 24

2.2.1. El método de los Hamiltonianos controlados . . . . . . . . . . . . . . . 24

2.2.2. Una versión particular . . . . . . . . . . . . . . . . . . 25

2.2.3. Las condiciones de matching cinéticas y potenciales . . . . . . . . . 27

2.2.4. Condiciones de matching simples . . . . . . . . . . . . . . . . . 30

2.3. El método LCB revisitado: maximalidad del LCB . . . . . . . . . . . . . . . . . 34

2.4. El método LCB para funciones simples . . . . . . . . . . . . . . . 35

2.4.1. Las ecuaciones cinéticas y potenciales . . . . . . . . . . . . . . . 35

2.4.2. El método LCB simple . . . . . . . . . . . . . . . . . . . 38

2.4.3. Maximalidad y equivalencia . . . . . . . . . . . . . . . . 40 
3. Estabilizabilidad asintótica de sistemas con dos grados de libertad 43

3.1. Ecuaciones cinéticas y potenciales para sistemas regularmente actuados . . . . . . 45

3.2. Estabilizabilidad de sistemas 1 -subactuados . . . . . . . . . . . . . . 50

3.2.1. Las ecuaciones cinéticas y potenciales para sistemas 1 -subactuados . . . . . 50

3.2.2. Una condición suficiente para la estabilizabilidad mediante el método LCB simple 51

3.2.3. Una condición necesaria para la existencia de una función de Lyapunov simple 59

3.2.4. Sistemas con dos grados de libertad . . . . . . . . . . . . . . . . . 60

3.3. Estabilizabilidad asintótica local . . . . . . . . . . . . . . . . 62

3.3.1. Sistemas con dos grados de libertad . . . . . . . . . . . . . . . . 62

3.3.2. Ejemplo: péndulo con disco invertido . . . . . . . . . . . . . 68

4. Soluciones por cuadraturas de las ecuaciones cinéticas y potenciales para sistemas 1-subactuados

4.1. Nueva expresión de las ecuaciones cinéticas y potenciales a partir de un cambio de variables dependientes . . . . . . . . . . . . . . . . . . . 72

4.1.1. Ecuaciones cinéticas y potenciales en las variables $(\delta, \gamma, l) \ldots \ldots \ldots$

4.1.2. Expresiones en coordenadas . . . . . . . . . . . . . . . . . . . 79

4.2. Integración por cuadraturas de las ecuaciones potenciales: actuación arbitraria . . . . 82

4.2.1. Elección del morfismo $\gamma \ldots \ldots \ldots$. . . . . . . . . . . . 83

4.2.2. Condiciones de integrabilidad de las ecuaciones potenciales . . . . . . . . 84

4.2.3. Condiciones de positividad . . . . . . . . . . . . . . . . . . 85

4.2.4. Construcción por cuadraturas de soluciones locales . . . . . . . . . . . . . 87

4.3. Soluciones por cuadraturas de las ecuaciones cinéticas para sistemas 1-subactuados . $\quad 89$

4.3.1. Positividad de las soluciones por cuadraturas para sistemas 1-subactuados . . 90

4.3.2. Coordenadas que rectifican $W \ldots \ldots \ldots \ldots$

5. Soluciones explícitas para sistemas 1-subactuados con simetría 97

5.1. Soluciones explícitas de las ecuaciones potenciales para una clase de sistemas $m$-subactuados . . . . . . . . . . . . . . . . . . 98

5.1.1. Una expresión alternativa de las ecuaciones potenciales . . . . . . . . . 98

5.1.2. Condiciones de integrabilidad local _. . . . . . . . . . . . . . . . 99

5.1.3. Condiciones de positividad para las soluciones de las ecuaciones potenciales . 100

5.1.4. Construcción de soluciones explícitas de las ecuaciones potenciales . . . . . . 101

5.2. Sistemas 1-subactuados con simetría . . . . . . . . . . . . . . . . . . . . . 103

5.2.1. Acciones de Grupos de Lie e invariancia . . . . . . . . . . . . . . . . . . 103

5.2.2. Cartas coordenadas $G$-adaptadas . . . . . . . . . . . . . . . . . . . . . 104

5.3. Soluciones explícitas para sistemas con simetría . . . . . . . . . . . . . . . 107

5.3.1. Soluciones de las ecuaciones cinéticas . . . . . . . . . . . . . . . . . 107 
5.3.2. Soluciones de las ecuaciones potenciales . . . . . . . . . . . . . . . . . 109

5.4. Ejemplo: péndulo doble invertido . . . . . . . . . . . . . . . . 112

5.4.1. Sistemas con dos grados de libertad . . . . . . . . . . . . . . . . . . . . . . . . . . . . . . . . . . . . . . .

5.4.2. El ejemplo . . . . . . . . . . . . . . . . . . . . . . . . 114

Apéndice

119 





\section{Gracias}

Es sábado. Me desperté hace unas tres horas y encendí la computadora. Preparé un mate y puse la radio, ansioso por terminar de escribir esta tesis que lleva ya 2163 días y contando. Después de varias correcciones, de comas que sobran, de puntos que faltan, de ecuaciones numeradas y no numeradas; sólo falta decir gracias. Acá va.

A Marcela y Sergio, gracias por la confianza, la contención y la libertad creativa. A Santiago, gracias por compartir parte de su tiempo y de su trabajo y por recibirme cada vez que fui a Bahía. Al Departamento de Matemática (FCE-UNLP), por prestarme un rincón para escribir.

Gracias Nico, por escuchar una y otra vez mis angustias, por pensar conmigo la manera en la que transitamos esta parte de nuestras vidas y por compartir todas las experiencias que nos atraviesan desde que nos sentamos diariamente a cinco metros de distancia.

Gracias Dani, Noe y Marian; por ayudarme constantemente a sobrellevar mi ineptitud burocrática, por acompañarme todos los almuerzos de los últimos años y por ceder la oreja de cuando en vez.

Gracias Maribel, por hacer que mi aterrizaje a la facultad fuese tan lindo, por compartir largas horas de charla y de planificaciones. Por ser mi amiga. Pero, por sobre todo, gracias por los abrazos, por poner el cuerpo y la risa.

Gracias Pochi, Vale y Marian; porque están desde el comienzo y por acompañar, quizá sin saberlo, todo este proceso. Por los carnavales, los sikus y los almuerzos. Por el amor y la amistad.

Gracias Juli por el aguante del último año, por ser la desconexión y el agite, por el riesgo de saltar sin conocer, por los abrazos rompehuesos y fundamentalmente por estar.

Gracias familia, por las visitas, por los llamados, por los besos y abrazos a la distancia y por el aguante incondicional. Gracias Marian, porque de alguna manera compartimos un lugar de pertenencia, y eso se siente y nos une.

A todos, porque esto que termina es una parte mía y por lo tanto parte suya. Salud! 



\section{Resumen}

El método basado en el Vínculo de Lyapunov (o método LCB por su sigla en inglés) es un método no lineal global para la estabilización asintótica de puntos de equilibrios no estables de sistemas mecánicos subactuados. Este método está basado en la imposición de un vínculo cinemático sobre el sistema no actuado, obteniéndose la señal de control para estabilizar al sistema como la fuerza de vínculo asociada a dicho vínculo. El método tiene la ventaja adicional de producir una función de Lyapunov para el sistema a lazo cerrado, probando de esta manera la estabilidad (asintótica) del sistema. Si nos restringimos a sistemas con Hamiltonianos simples (es decir, de la forma término cinético más término potencial) y si buscamos funciones de Lyapunov también simples, el método puede reducirse a resolver un sistema de ecuaciones diferenciales en derivadas parciales, llamadas ecuaciones cinéticas y ecuaciones potenciales, cuya incógnita es la función de Lyapunov mencionada anteriormente.

Este trabajo tiene tres objetivos distintos. En primer lugar, establecer la relación del método LCB con otros métodos de estabilización conocidos. En segundo lugar, explotar el contexto del método LCB para extender un resultado relacionado con la estabilizabilidad asintótica de sistemas con dos grados de libertad. Finalmente, dar una prescripción concreta y útil para construir soluciones locales de las ecuaciones cinéticas y potenciales y, por consiguiente, la señal de control buscada. 



\begin{abstract}
The Lyapunov constraint-based method (or LCB method for short) is a recent global non-linear method for the asymptotic stabilization of unstable equilibria of underactuated mechanical systems. It is based on the imposition of a kinematic constraint on the unactuated system, where the control law to stabilize the system is obtained as the related constraint force. The method has the additional advantage of producing a Lyapunov function for the closed-loop system, proving the (asymptotic) stability. If we restrict to simple Hamiltonian functions (meaning kinetic plus potential terms) and if we seek for simple Lyapunov functions as well, the method can be reduced to solving a set of partial differential equations, called the kinetic equations and the potential equations, whose unknown is the aforementioned Lyapunov function.

This work has three separate goals. First, to establish the relation between the LCB method and other well-known stabilization procedures. Second, to exploit the framework of the LCB method to improve a result concerning the asymptotic stabilizability of systems with two degrees of freedom. Finally, to give a concrete and useful prescription to construct explicit solutions of the kinetic and potential equations and, consequently, the control law.
\end{abstract}





\section{Introducción y organización de la tesis}

En la Referencia [19], fue presentado un método no lineal global para la estabilización de puntos de equilibrio inestables de sistemas mecánicos subactuados, el método basado en el Vínculo de Lyapunov (o método LCB). La estrategia general del método se basa en imponer un vínculo cinemático para lograr la estabilización del sistema considerado. Esta idea de "estabilización mediante vínculos" no es propia del LCB, sino que ya ha aparecido en otros trabajos (ver, por ejemplo, [21, 32, 37]) y en pocas palabras funciona de la siguiente manera: se busca un conjunto de vínculos cinemáticos para lograr un determinado comportamiento en las trayectorias del sistema. Luego, se obtiene la señal de control buscada como la fuerza de vínculo asociada.

En el caso del LCB, el vínculo utilizado es el denominado Vínculo de Lyapunov [19]. Dicho vínculo tiene la particularidad de producir una función de Lyapunov $V$ para el sistema a lazo cerrado, lo cual explica su nombre. El corazón del método consiste en encontrar la fuerza que implementa dicho vínculo.

A partir de su aparición, el método LCB ha sido desarrollado con mayor detalle en [22] y fue extendido para sistemas con efectos impulsivos en [10]. En [22] se estudian, entre otros, sistemas con fricción y sistemas Hamiltonianos con un único actuador y cuya función Hamiltoniana $H$ y función de Lyapunov $V$ son ambas simples, i.e. funciones que pueden expresarse como la suma de un término cinético $k$ y una función potencial $v$. Para este último tipo de sistemas, los autores prueban que una condición necesaria y suficiente para la existencia y unicidad de fuerzas de vínculo se reduce a resolver un sistema de ecuaciones diferenciales en derivadas parciales (PDEs) para la función $V$. Más aun, puede verse que dicho sistema se divide en dos conjuntos de PDEs, uno que tiene como incógnita al término cinético de $V$, consecuentemente llamadas ecuaciones cinéticas y otro que tiene como incógnita al término potencial, las llamadas ecuaciones potenciales (una vez que se fija una solución de las primeras). Vale la pena mencionar que las soluciones buscadas deben ser definidas positivas. En el mismo artículo, se presentan varios ejemplos en los cuales se encuentran soluciones para estas ecuaciones. Sin embargo, no se realiza un estudio sistemático de existencia de soluciones.

Existen en la literatura otros métodos no lineales globales para la estabilización asintótica de sistemas subactuados que también consisten en resolver un sistema de PDEs. Entre ellos está, por ejemplo, 
la familia de métodos englobadas bajo el nombre de energy shaping [2, 4, 5, 6, 7, 15, 28, 35, 36, 38, 39], también conocido genéricamente con el nombre de IDA-PBC o método de los Hamiltonianos controlados [11, 35] (o su contraparte lagrangiana, el método de los Lagrangianos controlados [6, 7]). Dicho método se basa, a diferencia del LCB, en la idea de equivalencia por feedback de sistemas mecánicos. En este caso, las incógnitas de las PDEs características son los términos cinético y potencial de un nuevo Hamiltoniano (llamado Hamiltoniano controlado) y puede verse que las mismas también se dividen en dos conjuntos: las llamadas condiciones de matching cinéticas, cuya incógnita es el término cinético y las condiciones de matching potenciales, cuya incógnita es el término potencial (una vez que se fija una solución de la primera). En la referencia [22], se demuestra que éste método puede ser visto como un caso particular del método basado en el vínculo de Lyapunov.

Dado que las condiciones de matching cinéticas tienen como incógnita únicamente al término cinético, una estrategia posible es estudiar primero esta ecuación y luego las condiciones de matching potenciales pensando que el término cinético es un dato de la segunda. Esta idea es trabajada en [30], donde se encuentran condiciones de integrabilidad para las condiciones de matching potenciales utilizando la teoría de integrablidad de Goldschmidt [17]. Como consecuencia, si las funciones involucradas en las ecuaciones son analíticas y si se cumplen estas condiciones de integrabilidad, este resultado dice que existen soluciones (analíticas) para las ecuaciones. Sin embargo, no se estudia la positividad de dichas soluciones.

Casi simultáneamente con la aparición de [22], una mejora del energy shaping para sistemas subactuados, definidos por funciones Lagrangianas o Hamiltonianas simples, fue introducida por Chang en [12, 13, 14]. Tal mejora consiste en una importante simplificación de las condiciones de matching. Gracias a esta simplificación, Chang logra probar condiciones suficientes para la estabilización de sistemas con $n-1$ actuadores y $n$ grados de libertad. Adicionalmente, en [13], muestra dos condiciones adicionales bajo las cuales un sistema subactuado con dos grados de libertad es asintóticamente estable.

Tanto en los trabajos de Chang, como en la referencia [30], el estudio de existencia de las soluciones de matching se hace desde un punto de vista teórico, i.e. no se expone un procedimiento concreto para la construcción de las mismas, sino que dichas soluciones se construyen en ejemplos específicos. Dicho procedimiento es de vital importancia en las aplicaciones a control automático, puesto que lo que se busca es la señal de control que realice la estabilización. Esta señal se construye, a su vez, a partir de soluciones de las condiciones de matching (en el caso del energy shaping) y de las ecuaciones cinéticas y potenciales (en el caso del LCB).

En términos generales, algunos objetivos de esta tesis son:

- Estudiar la relación del LCB con otros métodos de estabilización no lineal global en general y con el energy shaping en particular.

- Mostrar ejemplos de aplicación del método LCB para alcanzar estabilización asintótica de algunos sistemas subactuados 
- Construir procedimientos concretos que permitan encontrar soluciones locales definidas positivas de las ecuaciones cinéticas y potenciales.

A continuación damos una breve descripción de la organización de la tesis y de las contribuciones de la misma por cada capítulo:

Capítulo 1: En este capítulo introduciremos los conceptos que estarán presentes a lo largo de la tesis. Estableceremos la notación que utilizaremos en el resto del trabajo y recordaremos algunas definiciones básicas de Geometría Diferencial. También repasaremos algunas ideas de control de sistemas (sub)actuados. En particular nos interesará una subclase de estos sistemas: los sistemas Hamiltonianos subactuados. Por último presentaremos la idea de estabilización mediante vínculos y nos concentraremos en el método LCB. Para ellos tendremos que definir la noción de sistema Hamiltoniano con vínculos de segundo orden.

Capítulo 2: En primera instancia, precisaremos la idea de "método de estabilización". A partir de esta definición podremos introducir una relación de orden entre dichos objetos que nos permitirá, a su vez, hablar de equivalencia entre métodos. A partir de esta definición, mostraremos que el LCB es un método maximal, respecto de dicha relación de orden. A nuestro entender, estas definiciones no aparecen en ninguna literatura previa.

Luego, daremos un breve resumen del método energy shaping, en particular, de la versión desarrollada por Chang. Optamos por dar una descripción distinta de la tradicional (y por tanto novedosa) porque facilitará su comparación con el LCB. En el transcurso de esta descripción mostraremos la forma general de las fuerzas giroscópicas y disipativas, que incluyen a las utilizadas por Chang en [15] y daremos un algoritmo para construirlas. Este punto es crucial para probar la equivalencia con el método LCB simple.

Por último, extenderemos el estudio del método LCB (para funciones simples) y de sus PDEs asociadas a sistemas subactuados con más de un actuador y mostraremos además que este método es también maximal. Seguidamente, probaremos que las condiciones de Matching de Chang son exactamente las ecuaciones cinéticas y potenciales del LCB. Esto nos permitirá probar el resultado más importante del capítulo, a saber, que el método LCB para funciones simples es equivalente a la versión de Chang del energy shaping.

Capítulo 3: En la Referencia [12], D.E. Chang encontró condiciones suficientes que aseguran la estabilizabilidad de un sistema con $n$ grados de libertad y $n-1$ actuadores, por medio de la existencia de una función de Lyapunov (simple). Tal resultado se obtuvo estudiando la existencia de soluciones locales de las condiciones de matching. Además, en [13] se presentaron dos condiciones adicionales que garantizan que la estabilidad es asintótica si $n=1$. 
En este capítulo vamos a presentar una derivación alternativa de este resultado estudiando la existencia de soluciones locales de las ecuaciones cinéticas y potenciales del LCB. Más aun, debido a la equivalencia de ambos métodos y a la maximalidad del LCB, mostraremos que tales condiciones son de hecho necesarias si se pretende exhibir una función de Lyapunov simple para el sistema a lazo cerrado.

Para realizar este estudio introduciremos un cambio de variable dependiente que resultará en una simplifación importante de las ecuaciones cinéticas y potenciales.

Adicionalmente probaremos que, en el caso de sistemas con dos grados de libertad, la condición hallada en [12] no solamente asegura estabilizabilidad, sino también estabilizabilidad asintótica. En otras palabras, no es necesaria una condición adicional, además de la hallada en [12], para estabilizar asintóticamente un sistema Hamiltoniano subactuado con dos grados de libertad. Este es el resultado principal de este capítulo.

Capítulo 4: Como notamos anteriormente, no existe a nuestro entender un procedimiento sistemático que permita construir una solución explícita de las ecuaciones cinéticas y potenciales (o bien de las condiciones de matching). Este punto no es menor, puesto que el objetivo último de un método de estabilización es construir la señal de control que estabilice al sistema.

En este capítulo, presentaremos dos técnicas para encontrar explícitamente soluciones locales. Previamente, generalizaremos el cambio de variables dependientes del Capítulo 3 para simplificar las ecuaciones en el caso de sistemas con número arbitrario de actuadores.

El primer resultado de construcción da un procedimiento para hallar soluciones locales de las ecuaciones potenciales suponiendo que contamos con una solución de las ecuaciones cinéticas que además cumple con una condición de integrabilidad. Como dijimos antes, esta idea es también utilizada en [30]. Nuestro procedimiento, a diferencia de la referencia recién citada, es válido en la categoría $C^{\infty}$. Más aun, la solución puede computarse por cuadraturas (i.e. resolviendo integrales ordinarias) y, adicionalmente, mostramos una condición necesaria y suficiente para que la solución construida sea definida positiva.

El segundo resultado de construcción da un procedimiento para hallar soluciones de las ecuaciones cinéticas y potenciales para un subconjunto de sistemas con $n$ grados de libertad y $n-1$ actuadores que cumplen las restricciones del Capítulo 3. Para ello, mostramos cómo construir soluciones por cuadraturas de las ecuaciones cinéticas y luego aplicamos el resultado mencionado en el párrafo anterior para hallar soluciones de las ecuaciones potenciales. Esto nos permite dar un conjunto completo de instrucciones para construir soluciones locales de las ecuaciones cinéticas y potenciales, ambas computables por cuadraturas.

Capítulo 5: En este capítulo desarrollaremos un procedimiento para hallar soluciones explícitas de las ecuaciones cinéticas y potenciales para sistemas subactuados que presentan una simetría frente a la 
acción de un grupo de Lie $G$ que precisaremos más adelante.

En primer lugar, suponiendo que contamos con una solución de las ecuaciones cinéticas, trabajaremos con las ecuaciones potenciales para un sistema subactuado arbitrario. Suponiendo que cierto subfibrado vectorial del espacio de fases es integrable, encontraremos condiciones de integrabilidad que aseguran la existencia de soluciones locales de estas ecuaciones en las coordenadas que rectifican dicho subfibrado. Más aun, estas soluciones podrán ser calculadas computando integrales ordinarias en esas coordenadas. Además, estudiaremos bajo qué condiciones dichas soluciones son definidas positivas. Al final condensamos estos resultados en un procedimiento para construir soluciones.

En segundo lugar, trabajamos con sistemas 1-subactuados que presentan una simetría frente a la acción de un grupo de Lie. Para estos sistemas, demostramos que existen coordenadas en las cuales el término cinético del Hamiltoniano depende de una única coordenada, lo que nos permite hallar soluciones de las ecuaciones cinéticas con las mismas características, i.e. que dependen únicamente de esa coordenada. Seguidamente, mostramos que es posible combinar estos resultados con el procedimiento mencionado en el párrafo anterior para dar un conjunto completo de instrucciones para construir soluciones explícitas de las ecuaciones cinéticas y potenciales. En particular, mostramos un cambio de coordenadas específico que vincula ambas construcciones y hace posible esta combinación.

Por último, ilustramos estos resultados con un ejemplo: el péndulo doble invertido. 



\section{CONCEPTOS PRELIMINARES}

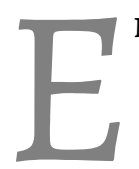

$\mathrm{N}$ este capítulo introduciremos los conceptos que estarán presentes a lo largo de la tesis. En primer lugar, estableceremos la notación que utilizaremos en el resto del trabajo y recordaremos algunas definiciones básicas de Geometría Diferencial. En segundo lugar, repasaremos ideas de control de sistemas (sub)actuados. En particular nos interesará una subclase de estos sistemas: los sistemas Hamiltonianos subactuados. Por último presentaremos la idea de estabilización mediante vínculos, para lo cual tendremos que definir la noción de sistema Hamiltoniano con vínculos de segundo orden.

Aclaración: En este trabajo supondremos que el lector posee conocimientos previos de Geometría Diferencial y Geometría Simpléctica [8, 29].

\subsection{Notación}

De ahora en adelante y salvo que se indique lo contrario, denotaremos por $Q$ a una variedad diferenciable ${ }^{1}$ y conexa de dimensión $n$. El fibrado tangente asociado a $Q$ será denotado $\tau_{Q}: T Q \rightarrow Q$ o simplemente $\tau$, cuando no exista riesgo de confusión. De manera similar, el fibrado cotangente será denotado $\pi_{Q}: T^{*} Q \rightarrow Q$, o simplemente $\pi$. Como es usual, denotaremos por $\langle\cdot, \cdot\rangle$ a la contracción natural entre $T_{q} Q$ y $T_{q}^{*} Q$ en cada $q \in Q$ y por $\mathfrak{X}(Q)$ y $\Omega^{1}(Q)$ a los haces de secciones de $\tau$ y $\pi$, respectivamente. También denotaremos por $\Omega^{k}(Q)$ al conjunto de $k$-formas diferenciales sobre $Q$ y escribiremos $\Omega^{\bullet}(Q)=\oplus_{n \in \mathbb{N}} \Omega^{n}(Q)$. El elemento neutro de cada espacio vectorial $T_{q} Q$ y $T_{q}^{*} Q$ será denotado por $0_{q}$.

Denotaremos por $\omega$ a la forma simpléctica canónica de la variedad simpléctica $T^{*} Q$. A partir de $\omega$, el corchete de Poisson canónico sobre $T^{*} Q$ es la asignación bilineal y antisimétrica $\{\}:, C^{\infty}\left(T^{*} Q\right) \times$

\footnotetext{
${ }^{1}$ Las palabras "diferenciable" y "suave" serán usadas como sinónimos en toda la tesis. Alternativamente diremos que un objeto diferenciable es de clase $C^{\infty}$ (o simplemente que el objeto es $C^{\infty}$ ).
} 
$C^{\infty}\left(T^{*} Q\right) \rightarrow C^{\infty}\left(T^{*} Q\right)$ dada por

$$
\{f, g\}=\omega(\mathrm{d} f, \mathrm{~d} g),
$$

donde $f, g \in C^{\infty}\left(T^{*} Q\right)$.

Una distribución sobre $Q$ consistirá en una asignación $\Delta: q \mapsto \Delta_{q}$, donde $\Delta_{q}$ es un subespacio vectorial de $T_{q} Q$ para cada $q \in Q$. Diremos que una distribución es suave (o $C^{\infty}$ o regular) si es localmente generada por secciones suaves de $T Q$. Si $\operatorname{dim} \Delta_{q}$ es independiente de $q$, diremos que $\Delta$ tiene rango constante. Del mismo modo, entenderemos por una codistribución $\Sigma$ sobre $Q$ al concepto canónicamente dual. Dado un campo vectorial $X \in \mathfrak{X}(Q)$ tal que $X(q) \in \Delta_{q}$ para todo $q \in Q$, diremos que $X$ toma valores en $\Delta$ y escribiremos $X \subset \Delta$ (usaremos una notación análoga en el caso de una forma diferencial $\omega \in \Omega^{1}(Q)$ y una codistribución $\Sigma$ ).

Si $P$ es otra variedad diferenciable de dimensión $m$ y $f: Q \rightarrow P$ es una función diferenciable entre $Q$ y $P$, denotaremos $f_{*}: T Q \rightarrow T P$ y $f^{*}: T^{*} P \rightarrow T^{*} Q$ a la aplicación tangente de $f$ y su transpuesta, respectivamente. En el caso de funciones a valores reales, usaremos también la notación $f_{*}=\mathrm{d} f$. Dado $q \in Q$, la notación $f_{* q}$ será utilizada para la aplicación lineal que resulta de restringir $f_{*}$ al espacio vectorial $T_{q} Q$, i.e.

$$
f_{* q}=\left.f_{*}\right|_{T_{q} Q}: T_{q} Q \rightarrow T_{f(q)} P .
$$

Un par $(P, \omega)$, donde $P$ es una variedad diferenciable y $\omega$ es una 2 -forma diferencial cerrada que es no degenerada en cada punto $p \in P$ se denomina variedad simpléctica.

Dado $q \in Q$ y un conjunto $U \subseteq Q$, diremos que $U$ es un entorno de $q$ si $U$ es abierto y además $q \in U$. También usaremos el término $q$-entorno para referirnos al conjunto $U$. Dada una función $f \in C^{\infty}(Q)$, decimos que $f$ es definida positiva alrededor de $q_{0} \in Q$ si existe un $q_{0}$-entorno $U$ tal que $f\left(q_{0}\right)=0 \mathrm{y}$ $f(q)>0$ para todo $q \in U \backslash\left\{q_{0}\right\}$.

Si $(U, \varphi)$ es una carta coordenada de $Q$, optaremos por escribir $\varphi(q)=\left(\varphi^{1}(q), \ldots, \varphi^{n}(q)\right)$ para cualquier punto $q \in Q$. Sobre las variedades $T Q$ y $T^{*} Q$ tenemos coordenadas asociadas a $(U, \varphi)$ dadas por $\left(T U, \varphi_{*}\right)$ y $\left(T^{*} U, \varphi^{-1^{*}}\right)$, donde $T U=\tau^{-1}(U)$ y $T^{*} U=\pi^{-1}(U)$. En particular, en el contexto de sistemas Hamiltonianos y Lagrangianos, usaremos la notación

$$
\varphi(q)=\left(q^{1}, \ldots, q^{n}\right)
$$

o simplemente

$$
\mathbf{q}=\left(q^{1}, \ldots, q^{n}\right)
$$

para denotar coordenadas de un punto $q$ en $Q$. De manera similar, dados $X \in T_{q} Q$ y $\xi \in T_{q}^{*} Q$ con $q \in U$, escribiremos

$\varphi_{*}(X)=\left(q^{1}, \ldots, q^{n}, \dot{q}^{1}, \ldots, \dot{q}^{n}\right)=(\mathbf{q}, \dot{\mathbf{q}}) \quad$ y $\quad\left(\varphi^{*}\right)^{-1}(\xi)=\left(q^{1}, \ldots, q^{n}, p_{1}, \ldots, p_{n}\right)=(\mathbf{q}, \mathbf{p})$

o simplemente

$$
\varphi_{*, q}(X)=\dot{\mathbf{q}} \quad \mathrm{y} \quad\left(\varphi_{q}^{*}\right)^{-1}(\xi)=\mathbf{p} .
$$


Una curva suave $\gamma$ en una variedad $Q$ es una aplicación diferenciable $\gamma:(-\varepsilon, \varepsilon) \rightarrow Q$, donde $(-\varepsilon, \varepsilon) \subset \mathbb{R}$. El vector tangente a una curva $\gamma$ sobre un punto $\gamma(t)$ de la misma es

$$
\dot{\gamma}(t)=\gamma_{*}\left(\left.\frac{\mathrm{d}}{\mathrm{d} s}\right|_{s=t}\right) \in T_{\gamma(t)} Q .
$$

$\operatorname{Si}(U, \varphi)$ es una carta coordenada de $Q$, denotaremos

$$
\gamma^{i}(t)=\varphi^{i}(\gamma(t)) \quad \text { y } \quad \dot{\gamma}^{i}(t)=\varphi_{*}^{i}(\dot{\gamma}(t))
$$

para todo $t$ tal que $\gamma(t) \in U$.

Dado $(\mathcal{U}, \Pi, Q)$ un fibrado vectorial de rango $m$, podemos considerar el fibrado vectorial $S^{2}\left(\mathcal{U}^{*}\right)$, i.e. el fibrado vectorial sobre $Q$ cuya fibra en $q$ es el producto tensorial simétrico de $\mathcal{U}_{q}^{*}$ con sí mismo. A su vez, $S_{+}^{2}\left(\mathcal{U}^{*}\right) \subset S^{2}\left(\mathcal{U}^{*}\right)$ es el conjunto de todos los $\left(\begin{array}{l}2 \\ 0\end{array}\right)$-tensores simétricos y definidos positivos. Puede verse que dicho conjunto es un subfibrado (no vectorial) de $S^{2}\left(\mathcal{U}^{*}\right)$. Una sección $\phi \in \Gamma\left(S_{+}^{2}\left(\mathcal{U}^{*}\right)\right)$ es una forma bilineal simétrica y definida positiva sobre $\mathcal{U}$. Asociada a esta forma bilineal, tenemos el morfismo de fibrados vectoriales $\phi^{\sharp}: \mathcal{U} \rightarrow \mathcal{U}^{*}$ dado por

$$
\left\langle\phi^{\sharp}(\alpha), \beta\right\rangle=\phi(\alpha, \beta), \quad \forall \beta \in \mathcal{U}_{\Pi(\alpha)}^{*} .
$$

El hecho de que $\phi$ es definida positiva implica que el morfismo $\phi^{\sharp}$ es de hecho un isomorfismo de fibrados vectoriales. Denotaremos la inversa como $\phi^{b}: \mathcal{U}^{*} \rightarrow \mathcal{U}$.

Dado un entorno abierto $U \subseteq Q$, un marco local (o frame local) $\left\{E_{1}, \ldots, E_{m}\right\}$ sobre $U$ para el fibrado $\mathcal{U}$, es un conjunto de $m$ secciones locales sobre $U$ tales que $\mathcal{U}_{q}=\operatorname{span}\left\{E_{1}(q), \ldots, E_{m}(q)\right\}$ para todo $q$ en $U$. Dado un marco $\left\{E_{1}, \ldots, E_{m}\right\}$ de $\mathcal{U}$, es posible ver que existe un marco $\left\{E^{1}, \ldots, E^{m}\right\}$ en el fibrado dual $\mathcal{U}^{*}$, llamado marco dual, que satisface

$$
\left\langle E^{i}(q), E_{j}(q)\right\rangle=\delta_{j}^{i}, \quad \forall q \in U,
$$

donde $\delta_{j}^{i}$ es el símbolo de Kronecker. Si $(U, \varphi)$ es una carta sobre $Q$, el conjunto $\left\{\partial / \partial q^{1}, \ldots, \partial / \partial q^{n}\right\}$ (respectivamente $\left\{\mathrm{d} q^{1}, \ldots, \mathrm{d} q^{n}\right\}$ ) define un marco local sobre $U$ para el fibrado $T Q$ (respectivamente un marco local sobre $U$ para el fibrado $T^{*} Q$ ), denominado marco coordenado (respectivamente comarco coordenado). Dichos marcos son duales entre sí.

Por último, en los cálculos que involucren contracciones entre tensores covariantes y contravariantes utilizaremos la convención de suma sobre índices repetidos para no recargar la notación.

\subsection{Conexiones afines sobre un fibrado vectorial y el isomorfismo $\beta$}

Dado un fibrado vectorial $(\mathcal{U}, \Pi, Q)$, una conexión afin para $\Pi$ es una aplicación $\nabla: \mathfrak{X}(Q) \times$ $\Gamma(\mathcal{U}) \rightarrow \Gamma(\mathcal{U})$ cumpliendo

$$
\text { I } \nabla_{X_{1}+f X_{2}} Y_{1}=\nabla_{X_{1}} Y_{1}+f \nabla_{X_{2}} Y_{1}
$$


II $\nabla_{X_{1}}\left(Y_{1}+Y_{2}\right)=\nabla_{X_{1}} Y_{1}+\nabla_{X_{1}} Y_{2}$

III $\nabla_{X_{1}}\left(f Y_{1}\right)=X_{1}(f) Y_{1}+f \nabla_{X_{1}} Y_{1}$

para todo $X_{1}, X_{2} \in \mathfrak{X}(Q), Y_{1}, Y_{2} \in \Gamma(\mathcal{U})$ y $f \in C^{\infty}(\mathcal{U})$.

Tomando coordenadas $(U, \varphi)$ para $Q$, un marco $\left\{E^{1}, \ldots, E^{n}\right\}$ de $\mathcal{U}$ definido sobre $U$ y un marco $\left\{F_{1}, \ldots, F_{n}\right\}$ de $T Q$ sobre $U$, podemos definir los símbolos de Christoffel $\Gamma_{i j}^{k}$ de la siguiente manera:

$$
\nabla_{F_{i}} E^{k}=\Gamma_{i j}^{k} E^{j}
$$

Cabe destacar que los símbolos de Christoffel dependen, no sólo de las coordenadas locales, sino también del marco escogido.

Fijemos una conexión afin $\nabla: \mathfrak{X}(Q) \times \Gamma(\mathcal{U}) \rightarrow \Gamma(\mathcal{U})$ sobre el fibrado $(\mathcal{U}, \Pi, Q)$. A partir de estos datos podemos definir un difeomorfismo $\beta: T \mathcal{U} \rightarrow \mathcal{U} \oplus T Q \oplus \mathcal{U}$ de la siguiente manera (ver Ref. [18]). Dado un elemento $V \in T \mathcal{U}$, consideremos la curva $W:(-\varepsilon, \varepsilon) \rightarrow \mathcal{U}$ que pasa por $\tau_{\mathcal{U}}(V)$ con velocidad $V$ en $s=0$, i.e. $W_{*}\left(\mathrm{~d} /\left.\mathrm{d} s\right|_{0}\right)=V$. Definamos entonces

$$
\beta(V):=\tau_{\mathcal{U}}(V) \oplus \Pi_{*}(V) \oplus \frac{D}{D s} W(0),
$$

donde $D / D s$ denota la derivada covariante asociada a $\nabla$. Fijando $q \in Q$ y un vector $X \in \mathcal{U}_{q}$ (i.e. $\Pi(X)=q)$, tenemos isomorfismos lineales

$$
\beta_{X}: T_{X} \mathcal{U} \rightarrow T_{q} Q \oplus \mathcal{U}_{q} \quad \text { y } \quad \beta_{X}^{-1}: T_{q} Q \oplus \mathcal{U}_{q} \rightarrow T_{X} \mathcal{U}
$$

dados por

$$
\beta_{X}(V):=\Pi_{*}(V) \oplus \frac{D}{D s} W(0) \quad \text { y } \quad \beta_{X}^{-1}(Y \oplus Z):=W_{*}\left(\mathrm{~d} /\left.\mathrm{d} s\right|_{0}\right),
$$

respectivamente, donde $W$ en la segunda ecuación es una curva en $\mathcal{U}$ tal que

$$
W(0)=X, \quad(\Pi \circ W)_{*}\left(\mathrm{~d} /\left.\mathrm{d} s\right|_{0}\right)=Y \quad \text { y } \quad \frac{D}{D s} W(0)=Z .
$$

Asociados a estos isomorfismos están sus aplicaciones transpuestas

$$
\beta_{X}^{*}: T_{q}^{*} Q \oplus \mathcal{U}_{q}^{*} \rightarrow T_{X}^{*} \mathcal{U} \quad \text { y } \quad \beta_{X}^{*-1}: T_{X}^{*} \mathcal{U} \rightarrow T_{q}^{*} Q \oplus \mathcal{U}_{q}^{*} .
$$

En términos de $\beta$, los subfibrados horizontal y vertical asociados a $\nabla$ en un punto $X \in \mathcal{U}_{q}$ son, respectivamente,

$$
\operatorname{Hor}_{X}:=\beta_{X}^{-1}\left(T_{q} Q \oplus 0\right) \quad \text { y } \quad \operatorname{Ver}_{X}:=\operatorname{ker} \Pi_{* X}=\beta_{X}^{-1}\left(0 \oplus \mathcal{U}_{q}\right)
$$

Es posible mostrar que la aplicación levantamiento vertical dada por

$$
\operatorname{vlift}_{X}: \mathcal{U}_{q} \rightarrow \operatorname{ker} \Pi_{* X}:\left.Z \longmapsto \frac{\mathrm{d}}{\mathrm{d} s}\right|_{0}(X+s Z)
$$


es un isomorfismo y está relacionado con $\beta_{X}^{-1}$ mediante la fórmula ${ }^{2}$

$$
\operatorname{vlift}_{X}(Z)=\beta_{X}^{-1}(0 \oplus Z) .
$$

Supongamos ahora que $\mathcal{U}=T^{*} Q$ y $\Pi=\pi_{Q}=\pi$. El difeomorfismo asociado

$$
\beta: T T^{*} Q \rightarrow T^{*} Q \oplus T Q \oplus T^{*} Q
$$

está dado por

$$
\beta(V)=\alpha \oplus \pi_{*}(V) \oplus \frac{D}{D s} w(0), \forall \alpha \in T^{*} Q, V \in T_{\alpha} T^{*} Q,
$$

donde $w:(-\varepsilon, \varepsilon) \rightarrow T^{*} Q$ es una curva que pasa por $\alpha$ en $s=0$ con velocidad $V$. Tomando una carta local $(U, \varphi)$ de $Q$, es fácil mostrar que

$$
\left(\varphi^{*-1} \times \varphi_{*} \times \varphi^{*-1}\right) \circ \beta \circ\left(\varphi^{*}\right)_{*}(\mathbf{q}, \mathbf{p}, \dot{\mathbf{q}}, \dot{\mathbf{p}})=(\mathbf{q}, \mathbf{p}) \oplus(\mathbf{q}, \dot{\mathbf{q}}) \oplus(\mathbf{q}, \dot{\mathbf{p}}+\Gamma(\mathbf{p}, \dot{\mathbf{q}})),
$$

o bien, omitiendo las funciones coordenadas $\varphi$ (como haremos siempre y cuando no genere confusión),

$$
\beta(\mathbf{q}, \mathbf{p}, \dot{\mathbf{q}}, \dot{\mathbf{p}})=(\mathbf{q}, \mathbf{p}) \oplus(\mathbf{q}, \dot{\mathbf{q}}) \oplus(\mathbf{q}, \dot{\mathbf{p}}+\Gamma(\mathbf{q}, \mathbf{p}, \dot{\mathbf{q}})),
$$

donde $\Gamma(\mathbf{q}, \mathbf{p}, \dot{\mathbf{q}})$ son los símbolos de Christoffel $\Gamma_{i l}^{k}(q)$ de $\nabla$ respecto de los marcos definidos por los campos vectoriales y formas coordenadas, i.e.

$$
\Gamma_{i}(\mathbf{q}, \mathbf{p}, \dot{\mathbf{q}})=\Gamma_{i l}^{k}(\mathbf{q}) p_{k} \dot{q}^{l} .
$$

Por otro lado, utilizando la relación entre el levantamiento vertical vlift ${ }_{\alpha}: T_{\pi(\alpha)}^{*} Q \rightarrow \operatorname{ker} \pi_{* \alpha}$ y el isomorfismo lineal $\beta_{\alpha}: T_{\alpha} T^{*} Q \rightarrow T_{\pi(\alpha)} Q \oplus T_{\pi(\alpha)}^{*} Q$ [ver Ecs. (1.3), (1.4) y (1.7)], todo vector vertical $Y_{\alpha} \in T_{\alpha} T^{*} Q$ puede identificarse con un único covector $y_{\alpha} \in T_{\pi(\alpha)}^{*} Q$ de la siguiente manera:

$$
Y_{\alpha}=\operatorname{vlift}_{\alpha}\left(y_{\alpha}\right)=\beta_{\alpha}^{-1}\left(0 \oplus y_{\alpha}\right)=\beta^{-1}\left(\alpha \oplus 0 \oplus y_{\alpha}\right) .
$$

En consecuencia, todo campo vectorial vertical $Y: T^{*} Q \rightarrow T T^{*} Q$ está determinado por un único morfismo de fibrados $y: T^{*} Q \rightarrow T^{*} Q$ tal que

$$
Y(\alpha)=\operatorname{vlift}_{\alpha}(y(\alpha))=\beta^{-1}(\alpha \oplus 0 \oplus y(\alpha)) .
$$

Definición 1.1. Dada una función $F: \mathcal{U} \rightarrow \mathbb{R}$, definimos la derivada a lo largo de la fibra y la derivada a lo largo de la base de $F$ como las aplicaciones fibra a fibra $\mathbb{F} F: \mathcal{U} \rightarrow \mathcal{U}^{*}$ y $\mathbb{B} F: \mathcal{U} \rightarrow T^{*} Q$ dadas por

$$
\langle\mathbb{F} F(X), Z\rangle=\left.\frac{\mathrm{d}}{\mathrm{d} s} F(X+s Z)\right|_{s=0}
$$

y

$$
\langle\mathbb{B} F(X), Y\rangle=\left.\frac{\mathrm{d}}{\mathrm{d} s} F(W(s))\right|_{s=0},
$$

respectivamente, donde $W:(-\varepsilon, \varepsilon) \rightarrow \mathcal{U}$ es una curva horizontal tal que

$$
W(0)=X, \quad(\Pi \circ W)_{*}\left(\mathrm{~d} /\left.\mathrm{d} s\right|_{0}\right)=Y \quad \text { y } \quad \frac{D}{D s} W(s)=0 .
$$

\footnotetext{
${ }^{2}$ Esto es cierto independientemente de la conexión $\nabla$.
} 
Notemos que $\mathbb{F} F$ es independiente de la conexión, pero $\mathbb{B} F$ no lo es.

Observación 1.1. De acuerdo con (1.6) y (1.7),

$$
\begin{aligned}
\langle\mathbb{F} F(X), Z\rangle & =\left\langle\mathrm{d} F(X), \operatorname{vlift}_{X}(Z)\right\rangle=\left\langle\mathrm{d} F(X), \beta_{X}^{-1}(0 \oplus Z)\right\rangle \\
& =\left\langle\beta_{X}^{*-1}(\mathrm{~d} F(X)), 0 \oplus Z\right\rangle .
\end{aligned}
$$

En la Proposición 1.2 (ver más adelante), también encontraremos una relación entre la derivada a lo largo de la base de $F$ y su diferencial d $F$.

Tomemos ahora el caso del fibrado cotangente sobre $Q$. Dada una función suave $F: T^{*} Q \rightarrow \mathbb{R}$, las derivadas a lo largo de la fibra y de la base de $F$ son los morfismos de fibrados $\mathbb{F} F: T^{*} Q \rightarrow T Q$ y $\mathbb{B} F: T^{*} Q \rightarrow T^{*} Q$, respectivamente, que en coordenadas locales se escriben

$$
(\mathbb{F} F(\mathbf{q}, \mathbf{p}))^{i}=\frac{\partial F}{\partial p_{i}}(\mathbf{q}, \mathbf{p})
$$

y

$$
(\mathbb{B} F(\mathbf{q}, \mathbf{p}))_{i}=\frac{\partial F}{\partial q^{i}}(\mathbf{q}, \mathbf{p})+\Gamma_{i l}^{k}(\mathbf{q}) \frac{\partial F}{\partial p_{l}}(\mathbf{q}, \mathbf{p}) p_{k} .
$$

Proposición 1.1. Si $F: \mathcal{U} \rightarrow \mathbb{R}$ es una función básica, i.e. una función para la cual existe $f: Q \rightarrow \mathbb{R}$ tal que $F=f \circ \Pi$, entonces

$$
\mathbb{F} F=0 \quad y \quad \mathbb{B} F=\mathrm{d} f \circ \Pi .
$$

Demostración. Dados $X, Z \in \mathcal{U}_{q}$ para algún $q \in Q$, i.e. $\Pi(X)=\Pi(Z)=q$, tenemos que

$$
\langle\mathbb{F} F(X), Z\rangle=\left.\frac{\mathrm{d}}{\mathrm{d} s} F(X+s Z)\right|_{s=0}=\left.\frac{\mathrm{d}}{\mathrm{d} s}(f \circ \Pi)(X+s Z)\right|_{s=0}=\left.\frac{\mathrm{d}}{\mathrm{d} s} f(q)\right|_{s=0}=0 .
$$

Por otro lado, tomando $Y \in T_{q} Q$ y una curva $W$ cumpliendo (1.14),

$$
\langle\mathbb{B} F(X), Y\rangle=\left.\frac{\mathrm{d}}{\mathrm{d} s} F(W(s))\right|_{s=0}=\left.\frac{\mathrm{d}}{\mathrm{d} s} f(\Pi(W(s)))\right|_{s=0}=\langle\mathrm{d} f(\Pi(X)), Y\rangle,
$$

como queríamos probar.

Los isomorfismos $\beta_{X}^{*-1}$ [ver Ec. (1.5)] dan origen a su vez al difeomorfismo

$$
\widetilde{\beta}: T^{*} \mathcal{U} \rightarrow \mathcal{U} \oplus T^{*} Q \oplus \mathcal{U}^{*},
$$

siendo $\widetilde{\beta}(\Sigma)=\beta_{X}^{*-1}(\Sigma)$, para todo $X \in \mathcal{U}$ y $\Sigma \in T_{X}^{*} \mathcal{U}$. En el caso del fibrado cotangente, tenemos el difeomorfismo

$$
\widetilde{\beta}: T^{*} T^{*} Q \rightarrow T^{*} Q \oplus T^{*} Q \oplus T Q .
$$

Proposición 1.2. Dada $F: \mathcal{U} \rightarrow \mathbb{R} y X \in \mathcal{U}$,

$$
\widetilde{\beta}(\mathrm{d} F(X))=X \oplus \mathbb{B} F(X) \oplus \mathbb{F} F(X) .
$$


Demostración. Tenemos que probar que, para todo $q \in Q, Y \in T_{q} Q$ y $Z \in \mathcal{U}_{q}$,

$$
\left\langle\beta_{X}^{*-1}(\mathrm{~d} F(X)), Y \oplus Z\right\rangle=\langle\mathbb{B} F(X), Y\rangle+\langle\mathbb{F} F(X), Z\rangle,
$$

o equivalentemente,

$$
\langle\mathrm{d} F(X), V\rangle=\langle\mathbb{B} F(X), Y\rangle+\langle\mathbb{F} F(X), Z\rangle, \quad \text { para } \quad V=\beta_{X}^{-1}(Y \oplus Z) .
$$

Sean $W_{1}:(-\varepsilon, \varepsilon) \rightarrow \mathcal{U}$ una curva cumpliendo (1.14) y $W_{2}:(-\varepsilon, \varepsilon) \rightarrow \mathcal{U}$ tal que $W_{2}(s):=X+s Z$. Dado que

$$
\beta_{X}\left(\left(W_{1}\right)_{*}\left(\mathrm{~d} /\left.\mathrm{d} s\right|_{0}\right)\right)=Y \oplus 0 \quad \text { y } \quad \beta_{X}\left(\left(W_{2}\right)_{*}\left(\mathrm{~d} /\left.\mathrm{d} s\right|_{0}\right)\right)=0 \oplus Z,
$$

tenemos que $\left(W_{1}\right)_{*}\left(\mathrm{~d} /\left.\mathrm{d} s\right|_{0}\right)+\left(W_{2}\right)_{*}\left(\mathrm{~d} /\left.\mathrm{d} s\right|_{0}\right)=V$. En consecuencia,

$$
\langle\mathrm{d} F(X), V\rangle=\left.\frac{\mathrm{d}}{\mathrm{d} s} F\left(W_{1}(s)\right)\right|_{s=0}+\left.\frac{\mathrm{d}}{\mathrm{d} s} F(X+s Z)\right|_{s=0},
$$

lo que concluye la prueba.

Sea $\omega$ la forma simpléctica canónica sobre $T^{*} Q$.

Proposición 1.3. Dados $\alpha \in T^{*} Q y V_{1}, V_{2} \in T_{\alpha} T^{*} Q$, yescribiendo $\beta_{\alpha}\left(V_{1}\right)=v_{1} \oplus \sigma_{1} y \beta_{\alpha}\left(V_{2}\right)=v_{2} \oplus \sigma_{2}$, tenemos que

$$
\omega\left(V_{1}, V_{2}\right)=\left\langle\sigma_{2}, v_{1}\right\rangle-\left\langle\sigma_{1}, v_{2}\right\rangle+\left\langle\alpha, T\left(v_{1}, v_{2}\right)\right\rangle,
$$

donde $T$ denota la torsión de $\nabla$.

Demostración. Fijando una carta coordenada local $(U, \varphi)$ que contenga a $q=\pi(\alpha)$ y escribiendo

$$
\varphi^{*-1}(\alpha)=(q, p) \quad \text { y } \quad\left(\varphi^{*-1}\right)_{*}\left(V_{\gamma}\right)=\left(q, p, \dot{q}_{\gamma}, \dot{p}_{\gamma}\right), \quad \gamma=1,2,
$$

es sabido que $\omega\left(V_{1}, V_{2}\right)=\left(\dot{p}_{2}\right)_{i}\left(\dot{q}_{1}\right)^{i}-\left(\dot{p}_{1}\right)_{i}\left(\dot{q}_{2}\right)^{i}$. Por otra parte, dado que

$$
\beta\left(q, p, \dot{q}_{\gamma}, \dot{p}_{\gamma}\right)=(q, p) \oplus\left(q, \dot{q}_{\gamma}\right) \oplus\left(q, \dot{p}_{\gamma}+\Gamma\left(p, \dot{q}_{\gamma}\right)\right)
$$

[ver (1.10)], tenemos que $\varphi_{*}\left(v_{\gamma}\right)=\left(q, \dot{q}_{\gamma}\right)$ y $\varphi^{*-1}\left(\sigma_{\gamma}\right)=\left(q, \dot{p}_{\gamma}+\Gamma\left(p, \dot{q}_{\gamma}\right)\right)$, y en consecuencia

$$
\begin{aligned}
\left\langle\sigma_{2}, v_{1}\right\rangle-\left\langle\sigma_{1}, v_{2}\right\rangle+\left\langle\alpha, T\left(v_{1}, v_{2}\right)\right\rangle= & \left(\dot{p}_{2}+\Gamma\left(q, p, \dot{q}_{2}\right)\right)_{i}\left(\dot{q}_{1}\right)^{i} \\
& -\left(\dot{p}_{1}+\Gamma\left(q, p, \dot{q}_{1}\right)\right)_{i}\left(\dot{q}_{2}\right)^{i}+p_{i} T^{i}\left(q, \dot{q}_{1}, \dot{q}_{2}\right) .
\end{aligned}
$$

Luego, sólo debemos probar que

$$
\begin{aligned}
\left(\dot{p}_{2}\right)_{i}\left(\dot{q}_{1}\right)^{i}-\left(\dot{p}_{1}\right)_{i}\left(\dot{q}_{2}\right)^{i}= & \left(\dot{p}_{2}+\Gamma\left(q, p, \dot{q}_{2}\right)\right)_{i}\left(\dot{q}_{1}\right)^{i} \\
& -\left(\dot{p}_{1}+\Gamma\left(q, p, \dot{q}_{1}\right)\right)_{i}\left(\dot{q}_{2}\right)^{i}+p_{i} T^{i}\left(q, \dot{q}_{1}, \dot{q}_{2}\right),
\end{aligned}
$$

o equivalentemente, $\Gamma_{i}\left(q, p, \dot{q}_{2}\right)\left(\dot{q}_{1}\right)^{i}-\Gamma_{i}\left(q, p, \dot{q}_{1}\right)\left(\dot{q}_{2}\right)^{i}=-p_{i} T^{i}\left(q, \dot{q}_{1}, \dot{q}_{2}\right)$. Pero

$$
T^{i}\left(q, \dot{q}_{1}, \dot{q}_{2}\right)=\Gamma_{k l}^{i}(q)\left(\dot{q}_{1}\right)^{k}\left(\dot{q}_{2}\right)^{l}-\Gamma_{k l}^{i}(q)\left(\dot{q}_{2}\right)^{k}\left(\dot{q}_{1}\right)^{l},
$$

de donde se sigue de manera sencilla el resultado que queríamos probar. 
De ahora en adelante, supongamos que $\nabla$ no tiene torsión. En términos de los difeomorfismos $\beta$ y $\widetilde{\beta}$ tenemos el siguiente resultado.

Proposición 1.4. Para todo $v \in T Q, \alpha, \sigma \in T^{*} Q$, sobre el mismo punto base,

$$
\beta \circ \omega^{\sharp} \circ \widetilde{\beta}^{-1}(\alpha \oplus \sigma \oplus v)=\alpha \oplus v \oplus(-\sigma) .
$$

Demostración. Siguiendo con la notación introducida en al proposición anterior, dado que $T=0$ ( $\nabla$ es sin torsión), tenemos que

$$
\omega\left(\beta_{\alpha}^{-1}\left(v_{1} \oplus \sigma_{1}\right), \beta_{\alpha}^{-1}\left(v_{2} \oplus \sigma_{2}\right)\right)=\left\langle\sigma_{2}, v_{1}\right\rangle-\left\langle\sigma_{1}, v_{2}\right\rangle,
$$

o equivalentemente

$$
\left\langle\left(\beta_{\alpha}^{*-1} \circ \omega^{b} \circ \beta_{\alpha}^{-1}\right)\left(v_{1} \oplus \sigma_{1}\right),\left(v_{2} \oplus \sigma_{2}\right)\right\rangle=\left\langle\sigma_{2}, v_{1}\right\rangle-\left\langle\sigma_{1}, v_{2}\right\rangle .
$$

Esto implica que $\left(\beta_{\alpha}^{*-1} \circ \omega^{b} \circ \beta_{\alpha}^{-1}\right)(v \oplus \sigma)=-\sigma \oplus v$ para todo $v, \sigma$ sobre el mismo punto base de $\alpha$. Finalmente, utilizando la identidad

$$
\left(\beta_{\alpha}^{*-1} \circ \omega^{b} \circ \beta_{\alpha}^{-1}\right)^{-1}=\beta_{\alpha} \circ \omega^{\sharp} \circ \beta_{\alpha}^{*},
$$

la prueba concluye.

Dado que el corchete de Poisson canónico sobre $T^{*} Q$ está dado por la fórmula

$$
\{F, G\}(\alpha)=\left\langle\mathrm{d} F(\alpha), \omega^{\sharp}(\mathrm{d} G(\alpha))\right\rangle, \quad \forall F, G \in C^{\infty}\left(T^{*} Q\right),
$$

usando la última proposición y la Ec. (1.19) es fácil encontrar que

$$
\{F, G\}(\alpha)=\langle\mathbb{B} F(\alpha), \mathbb{F} G(\alpha)\rangle-\langle\mathbb{B} G(\alpha), \mathbb{F} F(\alpha)\rangle .
$$

Consideremos ahora la suma de Whitney de $k$ copias de $\mathcal{U}$, que denotaremos $\mathcal{U} \times \cdots \times \mathcal{U}$. Fijando una conexión afin sobre $\Pi$, existe una conexión inducida de manera natural por la que fijamos sobre $\mathcal{U}$. Luego, dada una función $F: \mathcal{U} \times \cdots \times \mathcal{U} \rightarrow \mathbb{R}$, sus derivadas a lo largo de la fibra y de la base

$$
\mathbb{F} F: \mathcal{U} \times \cdots \times \mathcal{U} \rightarrow \mathcal{U}^{*} \times \cdots \times \mathcal{U}^{*} \quad \text { y } \quad \mathbb{B} F: \mathcal{U} \times \cdots \times \mathcal{U} \rightarrow T^{*} Q
$$

estarán dadas por las fórmulas

$$
\left\langle\mathbb{F} F\left(X_{1}, \ldots, X_{k}\right),\left(Z_{1}, \ldots, Z_{k}\right)\right\rangle=\left.\frac{\mathrm{d}}{\mathrm{d} s} F\left(X_{1}+s Z_{1}, \ldots, X_{k}+s Z_{k}\right)\right|_{s=0}
$$

y

$$
\left\langle\mathbb{B} F\left(X_{1}, \ldots, X_{k}\right), Y\right\rangle=\left.\frac{\mathrm{d}}{\mathrm{d} s} F\left(W_{1}(s), \ldots, W_{k}(s)\right)\right|_{s=0},
$$

respectivamente, donde cada $W_{i}:(-\varepsilon, \varepsilon) \rightarrow \mathcal{U}$ es una curva horizontal tal que

$$
W_{i}(0)=X_{i}, \quad\left(\Pi \circ W_{i}\right)_{*}\left(\mathrm{~d} /\left.\mathrm{d} s\right|_{0}\right)=Y \quad \text { y } \quad \frac{D}{D s} W_{i}(s)=0 .
$$


Observación 1.2. Como de costumbre, un tensor sobre $\mathcal{U}$ es una función $T: \mathcal{U} \times \cdots \times \mathcal{U} \rightarrow \mathbb{R}$, sobre la suma de Whitney de copias de $\mathcal{U}$, que es multilineal cuando se restringe a cada fibra. Cuando escribamos $T\left(X_{1}, \ldots, X_{k}\right)$, dejaremos implícito que todos los $X_{i}$ 's están contenidos en la misma fibra de $\mathcal{U}$.

Consideremos ahora un tensor $\mathfrak{b}: T^{*} Q \times T^{*} Q \rightarrow \mathbb{R}$ y su forma cuadrática asociada

$$
\mathfrak{q}: T^{*} Q \rightarrow \mathbb{R}: \alpha \longmapsto \mathfrak{b}(\alpha, \alpha)
$$

Proposición 1.5. Para todo $\alpha, \sigma \in T^{*} Q$,

$$
\langle\mathbb{F} \mathfrak{b}(\alpha, \alpha),(\sigma, \sigma)\rangle=\langle\mathbb{F} \mathfrak{q}(\alpha), \sigma\rangle \quad y \quad \mathbb{B} \mathfrak{b}(\alpha, \alpha)=\mathbb{B} \mathfrak{q}(\alpha)
$$

Demostración. De acuerdo con (1.22) y (1.24),

$$
\langle\mathbb{F} \mathfrak{b}(\alpha, \alpha),(\sigma, \sigma)\rangle=\left.\frac{\mathrm{d}}{\mathrm{d} s} \mathfrak{b}(\alpha+s \sigma, \alpha+s \sigma)\right|_{s=0}=\left.\frac{\mathrm{d}}{\mathrm{d} s} \mathfrak{q}(\alpha+s \sigma)\right|_{s=0}=\langle\mathbb{F} \mathfrak{q}(\alpha), \sigma\rangle
$$

Por otro lado, dados $\alpha \in T_{q}^{*} Q$ y $v \in T_{q} Q$, consideremos una curva horizontal $w$ tal que $w(0)=\alpha$ y $(\pi \circ w)_{*}\left(\mathrm{~d} /\left.\mathrm{d} s\right|_{0}\right)=v$. Usando (1.23) y (1.24), tenemos que

$$
\langle\mathbb{B} \mathfrak{b}(\alpha, \alpha), v\rangle=\left.\frac{\mathrm{d}}{\mathrm{d} s} \mathfrak{b}(w(s), w(s))\right|_{s=0}=\left.\frac{\mathrm{d}}{\mathrm{d} s} \mathfrak{q}(w(s))\right|_{s=0}=\langle\mathbb{B} \mathfrak{q}(\alpha), v\rangle,
$$

lo cual prueba la segunda parte de (1.25).

\subsection{Sistemas dinámicos y estabilización tipo Lyapunov}

En general, entenderemos por sistema dinámico a un sistema físico cuyos estados evolucionan en el tiempo siguiendo alguna ley bien definida. Para nosotros, un estado estará representado por un punto en alguna variedad diferenciable $P$ que supondremos conexa, de manera que la evolución temporal estará dada por una curva suave $\gamma:(-\varepsilon, \varepsilon) \rightarrow P$, parametrizada por el tiempo $t \in(-\varepsilon, \varepsilon)$ y que cumpla la ley de evolución de nuestro sistema dinámico. Dicha ley estará determinada por un campo vectorial suave $X \in \mathfrak{X}(P)$ en el siguiente sentido: $\gamma(t)$ es evolución temporal del sistema dinámico si y sólo si satisface

$$
\dot{\gamma}(t)=X(\gamma(t)) .
$$

Las soluciones de estas ecuaciones se denominan trayectorias del sistema. Dicho de otra forma, las trayectorias del sistema dinámico son las curvas integrales del campo vectorial $X$.

Observación 1.3. Más precisamente, estos sistemas se denominan autónomos, pues el campo vectorial $X$ no depende explícitamente del tiempo.

La siguiente definición formaliza y resume los conceptos presentados en los párrafos anteriores. 
Definición 1.2. Un sistema dinámico es un $\operatorname{par}(P, X)$, donde $P$ es una variedad suave y conexa y $X \in$ $\mathfrak{X}(P)$ es un campo vectorial suave sobre $P$. Una trayectoria $\gamma(t)$ del sistema dinámico $(P, X)$ es una curva integral de $X$.

Dado un sistema dinámico $(P, X)$, un estado $\bar{\alpha} \in P$ tal que $X(\bar{\alpha})=0$ se denomina punto de equilibrio del sistema pues la única trayectoria pasando por $\bar{\alpha}$ es la curva constante $\gamma(t)=\bar{\alpha}$. Alternativamente, también diremos que $\bar{\alpha}$ es un punto crítico del campo $X$. Dado un punto de equilibrio $\bar{\alpha}$ del sistema, nos interesa saber si trayectorias que comienzan cercanas a $\bar{\alpha}$ se mantienen cercanas a medida transcurre el tiempo. Más aún, nos interesa saber si existen trayectorias que se acerquen indefinidamente a $\bar{\alpha}$. Estas ideas están plasmadas en la siguiente

Definición 1.3. Un punto de equilibrio $\bar{\alpha}$ de $(P, X)$ es

a) estable si para todo $\bar{\alpha}$-entorno $U$, existe un $\bar{\alpha}$-entorno $V$ tal que, si $\gamma(t)$ es una trayectoria del sistema, vale que

$$
\gamma(0) \in V \quad \Rightarrow \quad \gamma(t) \in U, \quad \forall t \geq 0,
$$

b) inestable si no es estable,

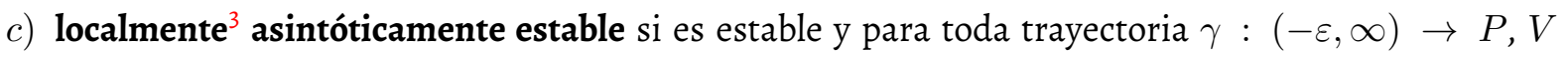
puede escogerse de manera tal que

$$
\gamma(0) \in V \quad \Rightarrow \quad \lim _{t \rightarrow \infty} \gamma(t)=\bar{\alpha} .
$$

En general, probar que un punto de equilibrio es (asintóticamente) estable puede ser una tarea muy complicada. No obstante, existe un resultado debido a Lyapunov que da una condición suficiente para determinar la estabilidad de un punto de equilibrio. Dicho resultado está basado en la existencia de una función diferenciable que introducimos en la siguiente definición.

Definición 1.4. Dados un punto de equilibrio $\bar{\alpha}$ de $(P, X)$ y un dominio $D \subset P$ que contiene a $\bar{\alpha}$, una función de Lyapunov para $\bar{\alpha}$ y $X$ es una función suave $V: D \rightarrow P$ cumpliendo las siguientes propiedades:

L1: $V(\bar{\alpha})=0$ y $V(\alpha)>0$ en $D-\{\bar{\alpha}\}$,

L2: $\langle\mathrm{d} V(\gamma(t)), \dot{\gamma}(t)\rangle \leq 0$, para toda trayectoria $\gamma(t)$ y todo tiempo $t$ tales que $\gamma(t) \in D$.

Teorema 1.1 (Lyapunov 1892). Sea $\bar{\alpha}$ un punto de equilibrio del sistema dinámico $(P, X)$. Entonces, si existe una función de Lyapunov $V$ para $\bar{\alpha} y$, el punto $\bar{\alpha}$ es estable.

Más aún, si la desigualdad en $\mathbf{L} 2$ es estricta, i.e.

$$
\langle\mathrm{d} V(\gamma(t)), \dot{\gamma}(t)\rangle<0 \text { en } D-\{\bar{\alpha}\},
$$

entonces $\bar{\alpha}$ resulta localmente asintóticamente estable.

Para la demostración ver [27].

\footnotetext{
${ }^{3}$ El punto de equilibrio se dice globalmente asintóticamente estable si puede tomarse $V=P$.
} 


\subsubsection{Estabilidad asintótica y el Principio de invariancia de LaSalle}

Como mencionamos al final de la sección anterior, el Teorema 1.1 es una poderosa herramienta para determinar el tipo de estabilidad de un punto de equilibrio. Si en particular queremos utilizar este resultado para probar estabilidad asintótica, es preciso encontrar una función de Lyapunov que cumpla (1.27). Sin embargo, esto no siempre es posible. Por suerte, existen otros criterios que nos permitirán estudiar este tipo de estabilidad. Entre ellos está el Principio de Invariancia de LaSalle, el cual permite determinar si un punto de equilibrio es asintóticamente estable estudiando los subconjuntos $X$-invariantes de $P$.

Definición 1.5. Un subconjunto $M \subset P$ se dice invariante por $X \circ X$-invariante si para toda trayectoria $\gamma(t)$ se cumple

$$
\gamma(0) \in M \Rightarrow \gamma(t) \in M \quad \forall t \in \mathbb{R} .
$$

Observación 1.4. Vale la pena notar que, en el caso en que $M$ es una subvariedad inmersa de $P, M$ es $X$-invariante si y sólo si $X(\alpha) \in T_{\alpha} M$ para todo $\alpha \in M$.

Teorema 1.2 (Principio de Invariancia de LaSalle). Sean $\bar{\alpha}$ un punto de equilibrio de $(P, X)$ y $V: D \rightarrow \mathbb{R}$ una función de Lyapunov para $\bar{\alpha} y X y$ denotemos

$$
\mathcal{S}_{0}:=\{\alpha \in D \mid\langle\mathrm{d} V(\alpha), X(\alpha)\rangle=0\} .
$$

Luego, si el conjunto unitario $\{\bar{\alpha}\}$ es el mayor subconjunto $X$-invariante de $\mathcal{S}_{0}$, el punto de equilibrio $\bar{\alpha}$ resulta localmente asintóticamente estable.

En realidad, el Principio de Invariancia puede enunciarse en un contexto más general, sin necesidad de contar con una función de Lyapunov. Sin embargo, este resultado es suficiente para los argumentos que presentaremos a lo largo de la tesis.

A continuación, introduciremos un algoritmo que permite encontrar el mayor subconjunto invariante de $\mathcal{S}_{0}$ y por lo tanto, via el Principio de Invariancia, nos ayudará a determinar si un dado punto de equilibrio es asintóticamente estable o no.

Lema 1.1. Sea $\bar{\alpha}$ un punto de equilibrio de $(P, X)$ y sea $V: D \rightarrow \mathbb{R}$ una función de Lyapunov para $\bar{\alpha}$ y $X$. Sea

$$
\mathcal{S}_{0}:=\{\alpha \in D \mid\langle\mathrm{d} V(\alpha), X(\alpha)\rangle=0\}
$$

y supongamos que $\mathcal{S}_{0}$ es una subvariedad de $P$, la llamaremos subvariedad de LaSalle. Sean los conjuntos

$$
\mathcal{S}_{n}:=\left\{\alpha \in \mathcal{S}_{n-1}: X(\alpha) \in T \mathcal{S}_{n-1}\right\}, \quad n \in \mathbb{N},
$$

para los cuales supondremos que cada $\mathcal{S}_{n}$ es una subvariedad de $\mathcal{S}_{n-1}$. Luego, el mayor subconjunto $X$-invariante I de $\mathcal{S}_{0}$ cumple

$$
\{\bar{\alpha}\} \subset I \subset \bigcap_{n \in \mathbb{N}} \mathcal{S}_{n} .
$$

En particular, si $\mathcal{S}_{k}=\{\bar{\alpha}\}$ para algún $k \in \mathbb{N}$, entonces $I=\{\bar{\alpha}\}$. 
La demostración de este lema es una consecuencia directa de la Observación 1.4.

En la siguiente sección introduciremos los sistemas que nos interesarán en el transcurso de la tesis, los sistemas Hamiltonianos.

\subsubsection{Sistemas Hamiltonianos}

Los sistemas dinámicos en los que estamos interesados se denominan sistemas Hamiltonianos. Tales sistemas se obtienen al considerar que $P$ es una variedad simpléctica con forma simpléctica $\omega$. En particular, el caso que nos interesa es aquél en el que $P=T^{*} Q$, donde $Q$ es una variedad diferenciable de dimensión $n$ y $\omega$ es la forma simpléctica canónica en $T^{*} Q$. En este caso, el campo vectorial $X$ está asociado a una función diferenciable $H: T^{*} Q \rightarrow \mathbb{R}$, denominada función Hamiltoniana del sistema o simplemente Hamiltoniano del sistema y se obtiene mediante la asignación [ver (1.1)]

$$
X=X_{H}=\omega^{\sharp} \circ \mathrm{d} H \text {. }
$$

El vector $X_{H}$ recibe el nombre de campo vectorial Hamiltoniano. Por lo dicho hasta aquí, es común denotar a estos sistemas por un par $(Q, H)$ antes que $\left(T^{*} Q, X_{H}\right)$, y así lo haremos de ahora en adelante. Como es bien conocido, alrededor de cualquier punto $\alpha \in T^{*} Q$ es posible hallar funciones coordena$\operatorname{das}^{4}(q, p)$ en las cuales $\omega=\mathrm{d} q^{i} \wedge \mathrm{d} p_{i}$. Utilizando las coordenadas inducidas en $T T^{*} Q$, es inmediato que $X_{H}$ admite la siguiente escritura

$$
\begin{aligned}
X_{H}(\mathbf{q}, \mathbf{p}) & =\frac{\partial H}{\partial p_{i}}(\mathbf{q}, \mathbf{p}) \frac{\partial}{\partial q^{i}}-\frac{\partial H}{\partial q^{i}}(\mathbf{q}, \mathbf{p}) \frac{\partial}{\partial p_{i}} \\
& =\left(\frac{\partial H}{\partial p_{1}}(\mathbf{q}, \mathbf{p}), \ldots, \frac{\partial H}{\partial p_{n}}(\mathbf{q}, \mathbf{p}),-\frac{\partial H}{\partial q^{1}}(\mathbf{q}, \mathbf{p}), \ldots,-\frac{\partial H}{\partial q^{n}}(\mathbf{q}, \mathbf{p})\right) .
\end{aligned}
$$

En el contexto de los sistemas Hamiltonianos, las ecuaciones (1.26) se conocen como ecuaciones de Hamilton. Utilizando el corchete canónico podemos expresar de una manera alternativa la acción del campo vectorial Hamiltoniano $X_{H}$ sobre una función suave $f$ del siguiente modo

$$
X_{H}(f)=\left\langle\mathrm{d} f, X_{H}\right\rangle=\left\langle\mathrm{d} f, \omega^{\sharp} \circ \mathrm{d} H\right\rangle=\{f, H\} .
$$

Dentro de los sistemas Hamiltonianos se encuentran los sistemas Hamiltonianos simples. Estos son sistemas en los cuales el Hamiltoniano adopta la forma

$$
H(\alpha)=\frac{1}{2}\left\langle\alpha, \rho^{\sharp}(\alpha)\right\rangle+\left(h \circ \pi_{Q}\right)(\alpha),
$$

donde $\rho$ es una métrica Riemanniana y $h \in C^{\infty}(Q)$. El primer término se conoce como término cinético mientras que el segundo se denomina término potencial.

Una revisión completa sobre sistemas Hamiltonianos y sus conceptos relacionados queda fuera del alcance de esta tesis. Nos limitaremos a referir al lector a las Referencias [1, 34] para una descripción más detallada de estos temas. No obstante, usaremos los resultados conocidos para estos sistemas sin mención específica.

\footnotetext{
${ }^{4}$ En el contexto más general de geometría simpléctica estas coordenadas son las llamadas coordenadas de Darboux.
} 


\subsection{El método de estabilización basado en el vínculo de Lyapunov}

En esta sección vamos a presentar un método global de estabilización no lineal que apareció por primera vez en [19] y fue desarrollado con más detalle en [22], llamado método de estabilización basado en el Vínculo de Lyapunov o método LCB por su nombre en inglés. Este método utiliza una idea relativamente reciente en la que la estabilización de sistemas subactuados se obtiene como consecuencia de la implementación de vínculos cinemáticos [22, 32, 33, 37]. La idea central de este tipo de métodos es construir una señal de control que estabilice un sistema mecánico dado que surja a su vez como la fuerza de vínculo asociada a un vínculo cinemático (escogido apropiadamente). En el caso del LCB, el vínculo en cuestión es el denominado Vínculo de Lyapunov [19, 22] y puede verse [22] que, para sistemas Hamiltonianos simples, dicho método se reduce a resolver un sistema de ecuaciones en derivadas parciales, cuya función incógnita es una función de Lyapunov que asegura la estabilidad del sistema. Estas ecuaciones son el objeto de estudio principal de este trabajo y serán presentadas hacia el final de la sección.

Para comenzar, definiremos dos tipos de sistemas que dan el marco teórico necesario para describir el método LCB: los sistemas Hamiltonianos con vínculos de segundo orden y los sistemas a lazo cerrado. Si bien muchas de estos tópicos admiten una descripción más general, en nuestra exposición nos restringiremos únicamente a los sistemas Hamiltonianos que presentamos en la sección anterior.

\subsubsection{Sistemas a lazo cerrado y sistemas con vínculos de segundo orden}

Consideremos la siguiente definición.

Definición 1.6. Dados un sistema Hamiltoniano $(Q, H)$ y una distribución vertical $\mathcal{W}$ sobre $T^{*} Q$, la familia de sistemas dinámicos dada por $\left(T^{*} Q, X_{H}+Y\right)$, donde $Y$ es un campo vectorial (la fuerza externa) que satisface

$$
Y \subset \mathcal{W}
$$

se denomina sistema Hamiltoniano actuado sobre $Q$ o sistema Hamiltoniano externamente forzado sobre $Q$ o simplemente sistema actuado sobre $Q$. Denotaremos a estos sistemas por un $\operatorname{par}(H, \mathcal{W})$. Una trayectoria de $(H, \mathcal{W})$ es una trayectoria de alguno de dichos sistemas dinámicos, i.e. una curva integral del campo $X_{H}+Y$ tal que $Y$ cumple (1.30).

La distribución $\mathcal{W}$ recibe el nombre de espacio de actuación. En los casos en los que la dimensión de las fibras $k$ de $\mathcal{W}$ sea estrictamente menor que $n$, diremos que el sistema es subactuado. Diremos también que $k$ es el grado de actuación (respectivamente $n-k$ es el grado de subactuación) y que el sistema es $k$-actuado (respectivamente $(n-k)$-subactuado).

El término "actuación" proviene del contexto de Teoría de Control y se refiere a la acción que ejercen ciertos dispositivos sobre un sistema mecánico para conseguir que sus trayectorias tengan un comportamiento determinado. Tales dispositivos reciben el nombre de actuadores y el espacio de actuación definido en 1.6 representa las direcciones posibles en las que éstos pueden ejercer fuerzas. 
Existen muchos comportamientos de interés que se pueden lograr sobre las trayectorias de un sistema mecánico mediante la implementación de actuadores sobre el mismo. Entre ellas se encuentra, por ejemplo, la estabilización asintótica de estados de equilibrio inestables. En este caso se busca que los actuadores produzcan una fuerza externa $Y$, de manera tal que las trayectorias del sistema dinámico dado por el par $\left(T^{*} Q, X_{H}+Y\right)$ sean asintóticamente estables en dicho estado. La fuerza $Y$ se denomina señal de control o bien ley de control y el diseño de la misma es un problema muy estudiado dentro de la Teoría de Control.

Definición 1.7 (CLMS). Dado un sistema Hamiltoniano actuado $(H, \mathcal{W})$ con espacio de actuación $\mathcal{W}$ y un campo vectorial $Y \subset \mathcal{W}$, el sistema mecánico a lazo cerrado (CLMS) con señal de control $Y$ es el sistema dinámico dado por el par $\left(T^{*} Q, X_{H}+Y\right)$. Denotaremos a estos sistemas por el par $(H, Y)$ o $(H, Y)_{\mathcal{W}}$ cuando queramos enfatizar el espacio de actuación involucrado.

Para una descripción más completa sobre sistemas a lazo cerrado y otros sistemas de interés dentro de la Teoría de Control, recomendamos consultar la referencia [9].

Otro tipo de sistemas dinámicos que nos interesarán son los sistemas mecánicos con vínculos cinemáticos de segundo orden. Estos son un caso particular de los sistemas Hamiltonianos con vínculos cinemáticos de orden superior o HOCS (ver [19]). Como veremos enseguida, estos sistemas están estrechamente relacionados con los CLMS y son el punto de partida de la estrategia de control que da origen al método LCB.

Definición 1.8 (SOCS). Dado un sistema Hamiltoniano $(Q, H)$, una subvariedad $\mathcal{P} \subset T T^{*} Q$ y una distribución vertical $\mathcal{W}$ sobre $T^{*} Q$, la familia de sistemas dinámicos dada por $\left(T^{*} Q, X\right)$, donde $X$ es un campo vectorial que satisface

$$
X \subset \mathcal{P} \quad \text { y } \quad X-X_{H} \subset \mathcal{W},
$$

se denomina sistema mecánico con vínculos cinemáticos de segundo orden o simplemente sistema con vínculos de segundo orden (SOCS). Denotaremos a estos sistemas por la terna $(H, \mathcal{P}, \mathcal{W})$. Una trayectoria de $(H, \mathcal{P}, \mathcal{W})$ es una curva integral de una solución $X$ de (1.31).

La subvariedad $\mathcal{P}$ se denomina subvariedad de vínculos y $\mathcal{W}$ es el subespacio de fuerzas de vínculo. El campo vectorial $Y=X-X_{H}$ se denomina fuerza de vínculo asociada a $X$.

Observación 1.5. Si $(q, p)$ son coordenadas locales en $T^{*} Q$ y tomamos las coordenadas inducidas correspondientes $(q, p, \dot{q}, \dot{p})$ en $T T^{*} Q$, sabemos que, al menos localmente, existen funciones $F^{1}, \ldots, F^{r} \in$ $C^{\infty}\left(\mathbb{R}^{4 n}\right)$ tales que $\mathcal{P}$ está descripta por

$$
F^{i}(\mathbf{q}, \mathbf{p}, \dot{\mathbf{q}}, \dot{\mathbf{p}})=0, \quad \forall i=1, \ldots, r .
$$

En otras palabras, la definición de la subvariedad de vínculos $\mathcal{P}$ involucra restricciones en las derivadas del momento $\dot{\mathbf{p}}$, lo cual justifica el nombre de vínculos de segundo orden. 
Observación 1.6. Notemos que $X$ es una solución de (1.31) si y sólo si $Y=X-X_{H}$ es solución de

$$
X_{H}+Y \subset \mathcal{P} \quad \text { y } \quad Y \subset \mathcal{W} .
$$

Observación 1.7. Siguiendo la Ec. (1.11), una distribución vertical $\mathcal{W}$ puede ser descripta por

$$
\mathcal{W}_{\alpha}=\beta_{\alpha}^{-1}\left(0 \oplus W_{\alpha}\right)
$$

donde $W_{\alpha}$ es un subespacio lineal de $T_{\pi(\alpha)}^{*} Q$.

Como comentamos anteriormente, los sistemas a lazo cerrado están estrechamente relacionados con los sistemas con vínculos. Hagamos explícita esta relación.

Tomemos un SOCS dado por una terna $(H, \mathcal{P}, \mathcal{W})$ y supongamos que existe único campo vectorial $X$ cumpliendo (1.31). Gracias a la Observación 1.6, esto es equivalente a decir que existe un único campo vectorial $Y$ cumpliendo (1.32). Este es el caso, por ejemplo, de un SOCS normal (ver [20]). Luego, las trayectorias de $(H, \mathcal{P}, \mathcal{W})$ son las curvas integrales del campo vectorial $X=X_{H}+Y$, donde $Y \subset \mathcal{W}$ es la fuerza de vínculo asociada a $X$. De esta manera, las trayectorias de $(H, \mathcal{P}, \mathcal{W})$ reproducen el comportamiento del CLMS $(H, Y)_{\mathcal{W}}$, pues las trayectorias de este CLMS son también las curvas integrales del campo $X_{H}+Y$. El campo vectorial $Y$, por otra parte, juega dos roles distintos: por un lado, es la fuerza de vínculo asociada al vínculo cinemático. Por el otro, es la señal de control del sistema a lazo cerrado. Así, el SOCS $(H, \mathcal{P}, \mathcal{W})$ da origen al CLMS $\left(H, X-X_{H}\right)_{\mathcal{W}}$. Esto nos dice que es posible diseñar estretegias de control para estabilizar sistemas subactuados a partir de sistemas con vínculos, i.e. podemos imponer sobre el sistema un conjunto apropiado de vínculos cinemáticos para lograr un comportamiento determinado, y obtener la señal de control como la fuerza de vínculo asociada.

En la referencia [20] se muestra que todo CLMS puede ser construido de esta manera, i.e. toda señal de control puede verse como la fuerza de vínculo de un conjunto de vínculos de segundo orden. Más precisamente, dada una señal de control de un sistema subactuado, existe un conjunto de vínculos de segundo orden tal que dicha señal es la fuerza de vínculo asociada. Este resultado revela una profunda conexión entre los CLMS y los sistemas con vínculos. Desde el punto de vista de las aplicaciones a control automático, este resultado dice que, a la hora de producir una ley de control para un sistema subactuado dado, siempre es posible "pensar en vínculos" (sin pérdida de generalidad).

\subsubsection{El Vínculo de Lyapunov y el método LCB}

Como presentamos en la Sección \$1.3.1, una manera de probar la estabilidad asintótica de un punto de equilibrio $\bar{\alpha}$ de un sistema dinámico $(P, X)$ es asegurando la existencia de una función de Lyapunov para $X$ y $\bar{\alpha}$. Introduciendo una función no negativa $\mu: P \rightarrow \mathbb{R}$ tal que $\mu(\bar{\alpha})=0$, la ecuación del punto L2 de la Definición 1.4 puede reescribirse

$$
\langle\mathrm{d} V(\gamma(t)), \dot{\gamma}(t)\rangle=-\mu(\gamma(t))
$$


En consecuencia, la condición $\mathbf{L 2}$ puede ser interpretada como un vínculo cinemático sobre el sistema. Siguiendo esta idea, si queremos estabilizar asintóticamente un sistema dinámico, podemos imponer la ecuación (1.34) como vínculo cinemático sobre el sistema, para funciones apropiadas $V$ y $\mu, \mathrm{y}$ obtener la señal de control que estabiliza al sistema como la fuerza de vínculo asociada (en este sentido y siguiendo con la idea presentada al final de la sección anterior, estamos controlando al sistema mediante la imposición de vínculos). Llamaremos a esta ecuación Vínculo de Lyapunov. Dado que la Ecuación (1.34) es una condición sobre las trayectorias del sistema, la misma se puede escribir en términos del campo $X$ como

$$
\langle\mathrm{d} V(\gamma(t)), X(\gamma(t))\rangle=-\mu(\gamma(t)) .
$$

Regresemos ahora a los sistemas Hamiltonianos, i.e. tomemos $P=T^{*} Q$ y consideremos un Hamiltoniano $H$. Tomando funciones no negativas $V, \mu: T^{*} Q \rightarrow \mathbb{R}$, impongamos el vínculo (1.34) sobre nuestro sistema. En otras palabras, definamos la subvariedad

$$
\mathcal{P}:=\left\{w \in T T^{*} Q:\left\langle\mathrm{d} V\left(\tau_{T^{*} Q}(w)\right), w\right\rangle=-\mu\left(\tau_{T^{*} Q}(w)\right)\right\},
$$

e impongamos el vínculo $\dot{\gamma}(t) \in \mathcal{P}$ sobre las trayectorias.

Observación 1.8. $\mathrm{Si}\left(q^{i}, p_{i}\right)$ denotan coordenadas locales sobre $T^{*} Q$, la subvariedad $\mathcal{P}$ puede ser descripta localmente mediante la ecuación

$$
\frac{\partial V}{\partial q^{i}}(\mathbf{q}, \mathbf{p}) \dot{q}^{i}+\frac{\partial V}{\partial p_{i}}(\mathbf{q}, \mathbf{p}) \dot{p}_{i}=-\mu(\mathbf{q}, \mathbf{p}) .
$$

Supongamos ahora que queremos implementar este vínculo ejerciendo fuerzas en las direcciones de una distribución vertical $\mathcal{W} \subset T T^{*} Q$. De este modo queda definido un SOCS $(H, \mathcal{P}, \mathcal{W})$. Supongamos ahora que este SOCS admite una solución $X$ (no necesariamente única) de (1.31), o equivalentemente, admite una fuerza de vínculo $Y$ cumpliendo (1.32) (En otras palabras, supongamos que el Vínculo de Lyapunov puese ser implementado por una fuerza de vínculo $Y \subset \mathcal{W}$ ). Esto es equivalente a decir que [ver Ec. (1.35) con $X=X_{H}+Y$ ]

$$
\left\langle\mathrm{d} V(\alpha), X_{H}(\alpha)+Y(\alpha)\right\rangle=-\mu(\alpha) \quad \text { e } \quad Y(\alpha) \in \mathcal{W},
$$

o bien,

$$
\mathfrak{i}_{X_{H}+Y} \mathrm{~d} V=-\mu \quad \text { e } \quad Y \subset \mathcal{W} .
$$

Dado que $\mu$ es no negativa, es claro que $\left\langle\mathrm{d} V(\alpha), X_{H}(\alpha)+Y(\alpha)\right\rangle \leq 0$, i.e. $V$ satisface la condición $\mathbf{L 2}$ de la Definición 1.4. Si además se cumple que $X_{H}(\bar{\alpha})+Y(\bar{\alpha})=0$ y $V$ satisface también la condición $\mathbf{L I}$ para $\bar{\alpha}$, entonces $V$ resulta ser una función de Lyapunov para $\bar{\alpha}$ y $X_{H}+Y$. En consecuencia, el sistema subactuado $(H, \mathcal{W})$ puede ser estabilizado en $\bar{\alpha}$ mediante la ley de control $Y$.

Observación 1.9. De esta manera, como vimos en la sección anterior, construímos un CLMS $(H, Y)_{\mathcal{W}}$ a partir del socs $(H, \mathcal{P}, \mathcal{W})$. 
En conclusión, si existe una solución $Y$ de la Ecuación (1.36), para algún par de funciones $V$ y $\mu$, es posible concluir diferentes cosas sobre la estabilizabilidad del sistema subactuado $(H, \mathcal{W})$ alrededor de $\bar{\alpha}$ [ver (1.27)], dependiendo de las características de las funciones $V$ y $\mu$.

Observación 1.10. Vale notar que, si $U \subset Q$ es un abierto de $Q$ y $\pi^{-1}(U) \subset T^{*} Q$ contiene al estado $\bar{\alpha}$, podemos hacer una afirmación análoga para una solución local $Y$ de (1.36), i.e. una solución a lo largo de $\pi^{-1}(U)$.

A partir del análsis efectuado en los párrafos anteriores podemos definir un método de estabilización (asintótica) de sistemas subactuados.

Definición 1.9. Dado un sistema subactuado $(H, \mathcal{W})$ sobre $Q$ y un punto crítico $\bar{\alpha} \in T^{*} Q$ de $X_{H}$, el método basado en el Vínculo de Lyapunov (LCB) consiste en hallar dos funciones $V, \mu: T^{*} Q \rightarrow \mathbb{R}$ y un campo vectorial $Y \in \mathfrak{X}\left(T^{*} Q\right)$ que cumplan la Ecuación (1.36) y tales que $V$ resulte definida positiva alrededor de $\bar{\alpha}, \mu$ sea no negativa e $Y(\bar{\alpha})=0$.

Dado que el Vínculo de Lyapunov está determinado por una única ecuación, es suficiente considerar una distribución $\mathcal{W}$ de rango 1 para generar la fuerza de vínculo asociada. En principio, esto quiere decir que es posible utilizar el método LCB para estabilizar un sistema subactuado con un grado de actuación únicamente. De aquí en adelante, y por el resto de este capítulo, supondremos entonces que

W1: $\mathcal{W}_{\alpha}=\operatorname{span}\{\Omega(\alpha)\}$, donde $\Omega$ es un campo vectorial vertical nunca nulo sobre $T^{*} Q$.

Además, dado que en la mayoría de las aplicaciones las direcciones de actuación no dependen de los momentos, también es razonable considerar

W2: $\Omega(\alpha)=\beta_{\alpha}^{-1}(0 \oplus \xi(\pi(\alpha)))$ [ver Ecs. (1.9) y (1.11)], con $\xi: Q \rightarrow T^{*} Q$.

Observación 1.11. Las suposiciones $\mathbf{W} \mathbf{1}$ y $\mathbf{W} \mathbf{2}$ dicen que $\mathcal{W}$ puede identificarse con un subfibrado vectorial $W \subset T^{*} Q$ de rango constante. Más precisamente, tenemos que [ver Ec. (1.33)]

$$
\mathcal{W}_{\alpha}=\beta_{\alpha}^{-1}\left(0 \oplus W_{\pi(\alpha)}\right)
$$

donde $W_{q}=\operatorname{span}\{\xi(q)\} \subset T^{*} Q$.

Suponiendo que $\mathcal{W}$ cumple las condiciones $\mathbf{W 1}$ y $\mathbf{W} \mathbf{2}$, podemos escribir $Y=\lambda \Omega$ para alguna función $\lambda \in C^{\infty}\left(T^{*} Q\right)$, de manera que la Ecuación (1.36) toma la forma

$$
\left\langle\mathrm{d} V(\alpha), X_{H}(\alpha)+\lambda(\alpha) \Omega(\alpha)\right\rangle=-\mu(\alpha) \quad \forall \alpha \in T^{*} Q .
$$

Luego, usando

$$
\left\langle\mathrm{d} V(\alpha), X_{H}(\alpha)\right\rangle=\{V, H\}(\alpha)
$$

y

$$
\langle\mathrm{d} V(\alpha), \Omega(\alpha)\rangle=\langle\xi(\pi(\alpha)), \mathbb{F} V(\alpha)\rangle,
$$


tenemos que

$$
\lambda(\alpha)=-\frac{\mu(\alpha)+\{V, H\}(\alpha)}{\langle\xi(\pi(\alpha)), \mathbb{F} V(\alpha)\rangle} .
$$

Por lo tanto, si existe una solución $Y$ de (1.36), ésta está determinada completamente por $Y=\lambda \Omega$ o bien, usando el difeomorfismo $\beta$,

$$
Y(\alpha)=\lambda(\alpha) \beta_{\alpha}^{-1}(0 \oplus \xi(\pi(\alpha))),
$$

donde $\lambda$ está definida por la Ec. (1.38). Es claro que la existencia de $Y$ está relacionada con el subconjunto

$$
\mathfrak{C}=\left\{\alpha \in T^{*} Q:\langle\xi(\pi(\alpha)), \mathbb{F} V(\alpha)\rangle=0\right\} .
$$

Por ejemplo, una condición necesaria para que $\lambda$ esté bien definida es

$$
\mu(\alpha)+\{V, H\}(\alpha)=0 \quad \forall \alpha \in \mathfrak{C} .
$$

Supongamos ahora que el Hamiltoniano $H$ es simple, i.e. está dado por

$$
H(\alpha)=\frac{1}{2}\left\langle\alpha, \rho^{\sharp}(\alpha)\right\rangle+\left(h \circ \pi_{Q}\right)(\alpha),
$$

donde $\rho$ es una métrica Riemanniana sobre $Q$ y $h \in C^{\infty}(Q)$. El primer término de $H$ se conoce como término cinético y el segundo es el término potencial.

Para encontrar condiciones suficientes que aseguren que $\lambda$ en (1.38) esté bien definida, consideraremos funciones de Lyapunov también simples, i.e.

$$
V(\alpha)=\frac{1}{2}\left\langle\alpha, \phi^{\sharp}(\alpha)\right\rangle+\left(v \circ \pi_{Q}\right)(q),
$$

donde $v \in C^{\infty}(Q)$ y $\phi$ es otra métrica Riemanniana sobre $Q$. En estas condiciones, tenemos que

$$
\mathbb{F} H=\rho^{\sharp} \quad \text { y } \quad \mathbb{F} V=\phi^{\sharp} .
$$

El siguiente Teorema es el resultado principal de esta sección

Teorema 1.3. Dado un sistema subactuado $(H, \mathcal{W})$, donde $H$ es un Hamiltoniano simple $y \mathcal{W}$ cumple las condiciones $\mathbf{W} 1$ y W2, existe una única solución $Y \subset \mathcal{W}$ de (1.36), con $V$ simple y $\mu$ no negativa, si y sólo si

$$
\{V, H\}(\alpha)=0, \quad \forall \alpha \in \mathfrak{C},
$$

$y$

$$
\frac{\mu}{\langle\xi(\pi(\cdot)), \mathbb{F} V(\cdot)\rangle} \in C^{\infty}\left(T^{*} Q\right) .
$$

La demostración de este teorema puede consultarse en [22].

A la luz de este resultado, para aplicar satisfactoriamente el método LCB, basta con tomar

$$
\mu(\cdot)=\varkappa\langle\xi(\pi(\cdot)), \mathbb{F} V(\cdot)\rangle^{2}, \quad \varkappa>0,
$$


y con encontrar una solución de

$$
\{V, H\}(\alpha)=0, \quad \forall \alpha \in \mathfrak{C},
$$

que es un sistema de ecuaciones diferenciales en derivadas parciales. Tenemos entonces la siguiente definición

Definición 1.10. Dado un sistema subactuado $(H, \mathcal{W})$ sobre $Q$, con $H$ un Hamiltoniano simple y $\mathcal{W}$ cumpliendo W1 y W2 y dado un punto crítico $\bar{\alpha} \in T^{*} Q$ de $X_{H}$, el método LCB simple para sistemas con un actuador consiste en hallar una función simple $V: T^{*} Q \rightarrow \mathbb{R}$ definida positiva alrededor de $\bar{\alpha}$ que cumpla la Ecuación (1.42).

Tomando coordenadas locales $(\mathbf{q}, \mathbf{p})$ alrededor de $\bar{\alpha}$, las funciones $H$ y $V$ se escriben

$$
H(\mathbf{q}, \mathbf{p})=\frac{1}{2} p_{i} \mathbb{H}^{i j}(\mathbf{q}) p_{j}+h(\mathbf{q})
$$

y

$$
V(\mathbf{q}, \mathbf{p})=\frac{1}{2} p_{i} \mathbb{V}^{i j}(\mathbf{q}) p_{j}+v(\mathbf{q})
$$

En las últimas expresiones, $\mathbb{H}$ y $\mathbb{V}$ son las representaciones matriciales coordenadas de las métricas Riemannianas definidas por los términos cinéticos de $H$ y $V$, respectivamente. Puede verse entonces que la Ecuación (1.42) se divide en dos sistemas de PDE, el primero

$$
\left(\frac{\partial \mathbb{V}^{i j}}{\partial q^{k}}(\mathbf{q}) \mathbb{H}^{k l}(\mathbf{q})-\frac{\partial \mathbb{H}^{i j}}{\partial q^{k}}(\mathbf{q}) \mathbb{V}^{k l}(\mathbf{q})\right) p_{i} p_{j} p_{l}=0
$$

que llamaremos ecuaciones cinéticas, el segundo

$$
\left(\frac{\partial v}{\partial q^{k}}(\mathbf{q}) \mathbb{H}^{k l}(\mathbf{q})-\frac{\partial h}{\partial q^{k}}(\mathbf{q}) \mathbb{V}^{k l}(\mathbf{q})\right) p_{l}=0,
$$

que llamaremos ecuaciones potenciales. Ambos sistemas deben cumplirse para todos los $(\mathbf{q}, \mathbf{p})$ tales que $\mathbf{p}^{t} \mathbb{V}(\mathbf{q}) \xi(\mathbf{q})=0$. Estos sistemas de PDEs son el objeto de estudio de esta tesis y serán analizados en los capítulos siguientes.

En la Referencia [22], las condiciones W1 y W2 son utilizadas en la prueba del Teorema 1.3. En el Capítulo 2, veremos que es posible extender el Teorema 1.3 al caso en el que hay más de un actuador, i.e. probaremos que es posible encontrar un conjunto de PDEs análogo a las Ecuaciones (1.44) y (1.45) para sistemas con grado de actuación arbitrario. 



\section{EQUIVALENCIA DE MÉTODOS DE}

\section{ESTABILIZACIÓN Y MAXIMALIDAD DEL MÉTODO LCB}

NA de las grandes ventajas del método LCB reside en que, no sólo produce la ley de control $Y$ buscada para estabilizar al sistema subactuado $(H, \mathcal{W})$ alrededor de un punto de equilibrio $\bar{\alpha}$, sino que además provee una función de Lyapunov $V$ para el campo $X_{H}+Y$ y el punto $\bar{\alpha}$. La existencia de dicha función es lo que muestra que el CLMS resultante $(H, Y)_{\mathcal{W}}$ es estable alrededor de $\bar{\alpha}$. Por supuesto, esta no es una característica exclusiva del LCB sino que, por el contrario, existen otros métodos de estabilización que también producen funciones de Lyapunov para el CLMS final. No obstante, dado que el Vínculo de Lyapunov nace de la definición misma de función de Lyapunov, es razonable preguntarse si tales métodos no están de algún modo contenidos en alguna versión del LCB.

En particular, existe una amplia variedad de métodos o procedimientos para alcanzar estabilidad asintótica de sistemas Langrangianos y Hamiltonianos subactuados englobados bajo el nombre de energy shaping, entre los cuales se cuentan: potential shaping, kinetic shaping, total energy shaping, energy plus force shaping, IDA-PBC, etc. Ver por ejemplo [2, 4, 5, 6, 7, 28, 35, 38, 39] o bien [15, 36] para trabajos más recientes. Dichos procedimientos están basados en la idea de equivalencia por feedback (ver Ref. [11]), y su propósito es construir, para un sistema subactuado dado, una ley de control y una función de Lyapunov para el CLMS resultante. Para ello, es preciso resolver un conjunto de ecuaciones diferenciales en derivadas parciales llamadas condiciones de matching, entre cuyas incógnitas se encuentra la función de Lyapunov que provee el método.

Todos los métodos arriba mencionados pueden verse como casos particulares del método de Lagrangianos controlados o bien del método de Hamiltonianos controlados (tal y como están definidos en la Referencia [11], donde además se muestra que ambos métodos son, en cierto sentido, equivalentes), y están caracterizados, esencialmente, por las correspondientes condiciones de matching. 
Casi simultáneamente con la aparición de [22], una mejora del energy (plus force) shaping para sistemas subactuados, definidos por funciones Lagrangianas o Hamiltonianas simples, fue introducida por Chang en [12, 13, 14]. Tal mejora consiste en una importante simplificación de las condiciones de matching. El resultado principal de este capítulo es la prueba de que tales ecuaciones son exactamente las ecuaciones cinéticas y potenciales del método LCB simple introducido en el capítulo anterior. Más aun, probaremos que la versión de Chang del energy shaping es equivalente (en un sentido que precisaremos más adelante) al método LCB simple. En otras palabras, lo que haremos en este capítulo es probar que la mejora del energy shaping introducida por Chang es precisamente el método LCB simple. Dado que el LCB simple fue desarrollado originalmente para sistemas subactuados con un único actuador, será necesario extender dicho método al caso de sistemas con grado de actuación arbitrario.

El capítulo está organizado de la siguiente manera. En primera instancia, en la Sección \$2.1, precisaremos la idea de "método de estabilización". A partir de esta definición podremos introducir una relación (de orden) entre dichos objetos que nos permitirá a su vez hablar de equivalencia entre métodos. A nuestro entender, estas definiciones no aparecen en literatura previa.

Luego, en la Sección \$2.2, daremos un breve resumen del método energy shaping utilizando la versión Hamiltoniana, i.e. el método de los Hamiltonianos controlados (o método $\mathrm{CH}$ por su nombre en inglés) o método IDA-PBC. Comenzaremos con una versión general del método que iremos particularizando paulatinamente hasta presentar la versión desarrollada por Chang (ver por ejemplo [15]) junto con sus correspondientes condiciones de matching. Vale la pena destacar que esta es una descripción novedosa del energy shaping, que usualmente es presentado a través de la noción de equivalencia por feedback. Optamos por esta nueva descripción porque facilitará la comparación con el método LCB, que es el objetivo general del capítulo. Además, en el transcurso de esta presentación, mostraremos también la forma general de las fuerzas giroscópicas y disipativas, que incluyen a las utilizadas por Chang en [15] y daremos un algoritmo para construirlas. Este punto es crucial para probar la equivalencia con el método LCB simple.

En las Secciones $\$ 2.3$ y $\$ 2.4$, extenderemos el estudio del método LCB simple y de sus PDEs asociadas a sistemas subactuados con más de un actuador y mostraremos que el método LCB es maximal con respecto a la relación de orden mencionada más arriba, en un sentido que será precisado más adelante. También probaremos que las condiciones de matching halladas por Chang son exactamente la ecuaciones cinéticas y potenciales del método LCB simple. Finalmente, mostraremos la equivalencia entre el LCB simple y el método CH en la versión de Chang. Este resultado es el principal del capítulo y es francamente inesperado teniendo en cuenta que el contexto en el que un método fue desarrollado difiere sustancialmente del contexto del otro. Por un lado, el método $\mathrm{CH}$ proviene de la idea de equivalencia por feedback de sistemas Hamiltonianos, por el otro, el método LCB se basa en la idea de estabilización mediante vínculos cinemáticos. Todos los resultados de estas dos secciones son contribuciones de esta tesis y fueron incluidos en la Referencia [23] junto con el resto de los resultados del capítulo. 


\subsection{Métodos de estabilización y equivalencia de métodos}

Fijemos una variedad $Q$, una función $H: T^{*} Q \rightarrow \mathbb{R}$ y un subfibrado vertical $\mathcal{W} \subset \operatorname{ker} \pi_{*} \subset T T^{*} Q$ del fibrado tangente sobre $T^{*} Q$. Denotemos por $X_{H}: T^{*} Q \rightarrow T T^{*} Q$ al campo vectorial Hamiltoniano asociado a $H$ con respecto a la estructura simpléctica canónica $\omega$ sobre $T^{*} Q$, i.e. $X_{H}:=\omega^{\sharp} \circ \mathrm{d} H \in$ $\mathfrak{X}\left(T^{*} Q\right)$. Fijemos también un punto crítico $\bar{\alpha} \in T^{*} Q$ de $X_{H}$, i.e. $X_{H}(\bar{\alpha})=0$. Recordemos que el par $(H, \mathcal{W})$ define un sistema Hamiltoniano subactuado sobre $Q$ (con función Hamiltoniana $H$ y espacio de actuadores $\mathcal{W}$ ). Supongamos que queremos resolver el siguiente problema.

P. Encontrar una señal de control $Y \subset \mathcal{W}$, i.e. un campo vectorial vertical $Y \in \mathfrak{X}\left(T^{*} Q\right)$ con valores en $\mathcal{W}$, tal que $Y(\bar{\alpha})=0$ y el sistema a lazo cerrado definido por $X_{H}+Y$ es estable en $\bar{\alpha}$.

Llamaremos método de estabilización a cualquier "procedimiento sistemático" que nos permita resolver el problema $\mathbf{P}$. Más precisamente, consideremos la siguiente definición

Definición 2.1. Sean $Q$ una variedad y $\mathfrak{U}$ un conjunto de ternas $(H, \mathcal{W}, \bar{\alpha})$, donde $(H, \mathcal{W})$ es un sistema subactuado sobre $Q$ y $\bar{\alpha} \in T^{*} Q$ un punto crítico de $X_{H}$. Dada una terna $(H, \mathcal{W}, \bar{\alpha}) \in \mathfrak{U}$, denotemos por $\mathcal{S}_{H, \mathcal{W}, \bar{\alpha}} \subset \mathfrak{X}\left(T^{*} Q\right)$ al subconjunto de campos vectoriales $Y \in \mathfrak{X}\left(T^{*} Q\right)$ que resuelven $\mathbf{P}$. Llamaremos método de estabilización sobre $\mathfrak{U}$ a cualquier función ${ }^{1} \digamma$ con dominio $\mathfrak{U}$ y con valores en el conjunto de partes de $\mathfrak{X}\left(T^{*} Q\right)$, tal que $\digamma(H, \mathcal{W}, \bar{\alpha}) \subset \mathcal{S}_{H, \mathcal{W}, \bar{\alpha}}$. Diremos además que $\digamma$ es de Lyapunov si para cada elemento $Y \in \digamma(H, \mathcal{W}, \bar{\alpha})$ puede exhibirse una función de Lyapunov para $X_{H}+Y$ y $\bar{\alpha}^{2}$

Observación 2.1. El objetivo de esta definición es dar un marco preciso para comparar distintos métodos de estabilización. Sin embargo, no construiremos la función $\digamma$ cuando describamos los métodos involucrados en la tesis, sino que nos limitaremos a dar una explicación sintética del procedimiento al que dan origen.

Consideremos también la siguiente definición.

Definición 2.2. Dados dos métodos de estabilización $\digamma$ y $\digamma^{\prime}$ sobre los subconjuntos $\mathfrak{U}$ y $\mathfrak{U}^{\prime}$, respectivamente, diremos que $\digamma$ está contenido en $\digamma^{\prime}$ si

$$
\digamma(H, \mathcal{W}, \bar{\alpha}) \subset \digamma^{\prime}(H, \mathcal{W}, \bar{\alpha}), \quad \forall(H, \mathcal{W}, \bar{\alpha}) \in \mathfrak{U} \cap \mathfrak{U}^{\prime}
$$

Si vale también la inclusión opuesta, diremos que $\digamma$ y $\digamma^{\prime}$ son equivalentes sobre $\mathfrak{U} \cap \mathfrak{U}$.

Observación 2.2. La relación de inclusión que acabamos de presentar define un orden parcial en el conjunto de todos los métodos de estabilización sobre un mismo $\mathfrak{U}$ dado. Vale la pena mencionar que un elemento maximal para este orden es un método de estabilización que es capaz de producir cualquier

\footnotetext{
${ }^{1}$ Notemos que un método de estabilización sobre $\mathfrak{U}$ puede ser visto también como una relación en $\mathfrak{U} \times \mathfrak{X}\left(T^{*} Q\right)$.

${ }^{2}$ El Teorema de Massera [31] (junto con sus generalizaciones -consultar [26]-) asegura que, si un sistema a lazo cerrado es asintóticamente estable, entonces existe una función de Lyapunov suave para tal sistema. Sin embargo, no puede asegurarse lo mismo si dicho sistema es solamente estable (ver [3]).
} 
ley de control construida a partir de cualquier otro método (sobre el mismo $\mathfrak{U}$ ). En este sentido, es claro que un tal método representa la manera más general de estabilizar sistemas subactuados en $\mathfrak{U}$ de manera sistemática.

Una observación análoga puede hacerse para el conjunto de métodos de estabilización de Lyapunov sobre un mismo $\mathfrak{U}$ dado.

\subsection{El método energy shaping}

En esta sección presentaremos la versión Hamiltoniana del energy shaping: el método de los Hamiltonianos controlados (como fue definido en [11]), también conocido como método IDA-PBC [35]. Describiremos una versión bastante general del método, junto con las condiciones de matching relacionadas, y lo haremos utilizando una terminología distinta a la usada en la literatura original con el objetivo de establecer la conexión con el LCB de manera más transparente. Por ejemplo, nos concentraremos en sistemas Hamiltonianos sobre un fibrado cotangente únicamente. De manera progresiva, iremos tomando en cuenta situaciones particulares hasta llegar finalmente a la versión desarrollada por Chang en las Referencias [12]-[15].

\subsubsection{El método de los Hamiltonianos controlados}

En lo que sigue, vamos a definir un método de estabilización de Lyapunov en el conjunto de todas las ternas $(H, \mathcal{W}, \bar{\alpha})$, conocido como el método de los Hamiltonianos controlados.

Supongamos que tenemos una función $V \in C^{\infty}\left(T^{*} Q\right)$, un tensor antisimétrico $B: T^{*} T^{*} Q \times$ $T^{*} T^{*} Q \rightarrow \mathbb{R}$ (en otras palabras, una estructura quasi-Poisson) sobre $T^{*} Q$ y dos campos vectoriales verticales $Z_{g}, Z_{d} \in \mathfrak{X}\left(T^{*} Q\right)$ tales que:

1. $V$ es definida positiva alrededor de $\bar{\alpha}$,

2. $\left\langle\mathrm{d} V(\alpha), Z_{g}(\alpha)\right\rangle=0$ para todo $\alpha \in T^{*} Q$, i.e. $Z_{g}$ es una fuerza giroscópica,

3. $B^{\sharp} \circ \mathrm{d} V+Z_{g}-X_{H} \subset \mathcal{W}$,

4. $\left\langle\mathrm{d} V(\alpha), Z_{d}(\alpha)\right\rangle \leq 0$ para todo $\alpha \in T^{*} Q$, i.e. $Z_{d}$ es una fuerza disipativa,

5. $Z_{d} \subset \mathcal{W}, \mathrm{y}$

6. $Z_{d}(\bar{\alpha})=-Z_{g}(\bar{\alpha})-B^{\sharp} \circ \mathrm{d} V(\bar{\alpha})$,

Notemos que el punto 1 implica $\mathrm{d} V(\bar{\alpha})=0$. Luego, definiendo $\hat{X}_{V}:=B^{\sharp} \circ \mathrm{d} V$ y $\hat{X}:=\hat{X}_{V}+$ $Z_{g}+Z_{d}$, el punto $\bar{\alpha}$ es un punto crítico de $\hat{X}$, gracias al punto 6, y para todo $\alpha \in T^{*} Q$

$$
\langle\mathrm{d} V(\alpha), \hat{X}(\alpha)\rangle=\left\langle\mathrm{d} V(\alpha), \hat{X}_{V}(\alpha)+Z_{g}(\alpha)+Z_{d}(\alpha)\right\rangle=\left\langle\mathrm{d} V(\alpha), Z_{d}(\alpha)\right\rangle \leq 0,
$$


debido a los puntos 2 y 4 y al hecho de que $\left\langle\mathrm{d} V(\alpha), \hat{X}_{V}(\alpha)\right\rangle=B(\mathrm{~d} V(\alpha), \mathrm{d} V(\alpha))=0$. En consecuencia, $V$ es una función de Lyapunov para el sistema dinámico definido por $\hat{X}$ y el punto crítico $\bar{\alpha}$, puesto que el punto 1 implica L1 y la Ec. (2.1) implica L2 (ver Teorema 1.1). Esto dice que dicho sistema es estable en $\bar{\alpha}$ (ver Definición 1.3). Luego, definiendo el campo

$$
Y:=\hat{X}-X_{H}
$$

que toma valores en $\mathcal{W}$ debido a los puntos 3 y 5 , hemos resuelto el problema $\mathbf{P}$. En particular, tenemos que

$$
\left\langle\mathrm{d} V(\alpha), X_{H}(\alpha)+Y(\alpha)\right\rangle \leq 0
$$

Todo esto da origen al siguiente procedimiento.

Definición 2.3. Dados un sistema subactuado $(H, \mathcal{W})$ sobre $Q$ y un punto crítico $\bar{\alpha} \in T^{*} Q$ de $X_{H}$, el método de los Hamiltonianos controlados (CH) consiste en hallar $V, B$ y $Z_{g}$ cumpliendo 1 y 2 junto con la ecuación [recordar el punto 3]

$$
B^{\sharp} \circ \mathrm{d} V+Z_{g}-\omega^{\sharp} \circ \mathrm{d} H \subset \mathcal{W} ;
$$

encontrar además un campo $Z_{d}$ cumpliendo los puntos 4, 5 y 6; y definir [ver (2.2)]

$$
Y:=B^{\sharp} \circ \mathrm{d} V+Z_{g}+Z_{d}-\omega^{\sharp} \circ \mathrm{d} H .
$$

Observación 2.3. Notemos que, dado que $X_{H}(\bar{\alpha})=\hat{X}(\bar{\alpha})=0$, tenemos que $Y(\bar{\alpha})=0$ [ver Ec. (2.2)].

Es claro entonces que el procedimiento descripto arriba define, de acuerdo con la Definición 2.1, un método de estabilización de Lyapunov: la función $\digamma$ correspondiente asigna a cada terna $(H, \mathcal{W}, \bar{\alpha})$ un conjunto de campos vectoriales $\digamma(H, \mathcal{W}, \bar{\alpha}) \subset \mathfrak{X}\left(T^{*} Q\right)$ dado por la Ec. (2.5) (y que por lo tanto resuelven $\mathbf{P}$ ), donde $V, B, Z_{g}$ y $Z_{d}$ deben cumplir las condiciones enunciadas en la última definición.

Observación 2.4. La manera usual con la que se introduce el método $\mathrm{CH}$ es a través de la idea de equivalencia por feedback [11].

\subsubsection{Una versión particular}

La esencia del método $\mathrm{CH}$ reside en la Ec. (2.4), que es un sistema de PDEs para $V$, con "parámetros" $B$ y $Z_{g}$ a determinar. Estas ecuaciones diferenciales en derivadas parciales reciben el nombre de condiciones de matching. ${ }^{3}$ Haciendo distintas suposiciones sobre el sistema subactuado original $(H, \mathcal{W})$, y tomando diferentes ansatz para las incógnitas $V, B$ y $Z_{g}$, encontramos distintos casos particulares para la Ec. (2.4) y, en consecuencia, distintas versiones particulares del método CH. (En términos de la

\footnotetext{
${ }^{3}$ El resto de las ecuaciones, las condiciones de los puntos 4, 5 y 6, son condiciones algebraicas para $Z_{d}$ y serán estudiadas más adelante.
} 
Definición 2.2 y de la Observación 2.2, tenemos de esta manera una cadena ordenada de métodos de estabilización de Lyapunov.) Supongamos por ejemplo que $H, V: T^{*} Q \rightarrow \mathbb{R}$ son hiperregulares, i.e. $\mathbb{F} H, \mathbb{F} V: T^{*} Q \rightarrow T Q$ son isomorfismos de fibrados vectoriales [ver (1.13)], y además

$$
\mathbb{F} H=\mathbb{F} H^{*} \quad \text { y } \quad \mathbb{F} V=\mathbb{F} V^{*},
$$

i.e. sus derivadas a lo largo de la fibra son simétricas. Fijemos también una conexión sin torsión sobre $T^{*} Q$ y supongamos que [recordar Ecs. (1.8) y (1.18)]

$$
\beta \circ B^{\sharp} \circ \widetilde{\beta}^{-1}(\alpha \oplus \sigma \oplus v)=\alpha \oplus \Psi(v) \oplus \Psi^{*}(-\sigma),
$$

para algún morfismo de fibrados $\Psi: T Q \rightarrow T Q$ [comparar con la Ec. (1.20)].

Observación 2.5. Notemos que, de acuerdo con (1.11), cada $\mathcal{W}_{\alpha} \subset T_{\alpha} T^{*} Q$ está definido por el único subespacio $W_{\alpha} \subset T_{\pi(\alpha)}^{*} Q$ tal que

$$
\mathcal{W}_{\alpha}=\operatorname{vlift}_{\alpha}\left(W_{\alpha}\right)=\beta^{-1}\left(\alpha \oplus 0 \oplus W_{\alpha}\right) .
$$

Además [ver Ec. (1.12)], el campo vectorial vertical $Z_{g}$ puede ser escrito como

$$
Z_{g}(\alpha)=\beta^{-1}\left(\alpha \oplus 0 \oplus z_{g}(\alpha)\right),
$$

para un único morfismo de fibrados $z_{g}: T^{*} Q \rightarrow T^{*} Q$. Lo mismo puede decirse del campo $Z_{d}$.

Proposición 2.1. Teniendo en cuenta (2.6), (2.7), (2.8) y (2.9); las condiciones de matching (2.4) se reducen a

$$
\langle\mathbb{B} V(\alpha), \mathbb{F} H(\sigma)\rangle-\langle\mathbb{B} H(\alpha), \mathbb{F} V(\sigma)\rangle-\langle z g(\alpha), \mathbb{F} V(\sigma)\rangle=0
$$

donde [ver Ec. (2.8)]

$$
\hat{W}_{\alpha}:=\left(\mathbb{F} V\left(W_{\alpha}\right)\right)^{\circ}=\mathbb{F} V^{-1}\left(W_{\alpha}^{\circ}\right), \quad \forall \alpha \in T^{*} Q .
$$

En particular, las únicas incógnitas en las condiciones de matching son $V y z_{g}$.

Demostración. Teniendo en cuenta (1.19) y (2.7),

$$
\begin{aligned}
\beta\left(\hat{X}_{V}(\alpha)\right) & =\beta \circ B^{\sharp}(\mathrm{d} V(\alpha))=\beta \circ B^{\sharp} \circ \widetilde{\beta}^{-1}(\widetilde{\beta}(\mathrm{d} V(\alpha))) \\
& =\beta \circ B^{\sharp} \circ \widetilde{\beta}^{-1}(\alpha \oplus \mathbb{B} V(\alpha) \oplus \mathbb{F} V(\alpha)) \\
& =\alpha \oplus \Psi(\mathbb{F} V(\alpha)) \oplus \Psi^{*}(-\mathbb{B} V(\alpha)) .
\end{aligned}
$$

De manera similar, usando las Ecs. (1.19) y (1.20),

$$
\beta\left(X_{H}(\alpha)\right)=\alpha \oplus \mathbb{F} H(\alpha) \oplus(-\mathbb{B} H(\alpha)) .
$$

En consecuencia, utilizando las Ecuaciones (2.8), (2.9), (2.12) y (2.13), ; se sigue fácilmente que (2.4) adopta la forma

$$
\Psi(\mathbb{F} V(\alpha))=\mathbb{F} H(\alpha) \quad \text { y } \quad-\Psi^{*}(\mathbb{B} V(\alpha))+z_{g}(\alpha)+\mathbb{B} H(\alpha) \in W_{\alpha},
$$


para todo $\alpha \in T^{*} Q$. Esto implica que $\Psi=\mathbb{F} H \circ \mathbb{F} V^{-1}$ y [usando la Ec. (2.6)]

$$
-\mathbb{F} V^{-1} \circ \mathbb{F} H \circ \mathbb{B} V(\alpha)+z_{g}(\alpha)+\mathbb{B} H(\alpha) \in W_{\alpha} .
$$

En otras palabras, usando (2.11) tenemos que [ver Ec. (2.6) nuevamente]

$$
\langle\mathbb{B} V(\alpha), \mathbb{F} H(\sigma)\rangle-\langle\mathbb{B} H(\alpha), \mathbb{F} V(\sigma)\rangle-\left\langle z_{g}(\alpha), \mathbb{F} V(\sigma)\right\rangle=0,
$$

para todo $\alpha \in T^{*} Q, \sigma \in \hat{W}_{\alpha}$. Esto termina la prueba.

Observación 2.6. Utilizando la Ecuación (2.13), es claro que $\bar{\alpha}$ es un punto crítico de $X_{H}$ si y sólo si $\mathbb{F} H(\bar{\alpha})=0$ y $\mathbb{B} H(\bar{\alpha})=0$.

Vale la pena mencionar que, de acuerdo con las Ecs. (1.12) y (2.5), cada señal de control $Y$ del método se obtiene por medio del levantamiento vertical de la aplicación fibra a fibra

$$
y(\alpha):=z_{d}(\alpha)+z_{g}(\alpha)-\mathbb{F} V^{-1} \circ(\mathbb{F} H \circ \mathbb{B} V(\alpha)-\mathbb{F} V \circ \mathbb{B} H(\alpha)),
$$

donde

$$
\left\langle z_{g}(\alpha), \mathbb{F} V(\alpha)\right\rangle=0,
$$

usando que $z_{g}$ es giroscópica (el punto 2 arriba),

$$
\left\langle z_{d}(\alpha), \mathbb{F} V(\alpha)\right\rangle \leq 0 \quad \text { y } \quad z_{d}(\alpha) \in W_{\alpha},
$$

de acuerdo con los puntos 4 y $5, y$

$$
z_{d}(\bar{\alpha})=-z_{g}(\bar{\alpha})+\mathbb{F} V^{-1} \circ \mathbb{F} H \circ \mathbb{B} V(\bar{\alpha}),
$$

del punto 6.

\subsubsection{Las condiciones de matching cinéticas y potenciales}

Restrinjamos aún más la forma del sistema subactuado original $(H, \mathcal{W})$ y la forma de las incógnitas $V$ y $z_{g}$ de (2.10). Supongamos primero que $H: T^{*} Q \rightarrow \mathbb{R}$ es una función Hamiltoniana simple [recordar (1.39)], Notemos que $\mathfrak{h}$ es la forma cuadrática asociada al tensor

$$
\mathfrak{b}_{H}: T^{*} Q \times T^{*} Q \rightarrow \mathbb{R}:(\alpha, \sigma) \longmapsto \frac{1}{2}\left\langle\alpha, \rho^{\sharp}(\sigma)\right\rangle .
$$

También notemos que $\mathbb{F} H=\mathbb{F h}=\rho^{\sharp}$ [recordar la Ec. (1.17) de la Proposición 1.1]. Esto implica que $\mathbb{F} H$ es un isomorfismo de fibrados vectoriales. Tomando una carta coordenada $(U, \varphi)$ sobre $Q$ junto con su carta inducida sobre $T^{*} Q$, y denotando por $\mathbb{H}(\mathbf{q})$ la representación matricial coordenada en $\mathbf{q}$ de la métrica Riemanniana $\rho$ [ver (1.43)], la condición $\mathbb{F} H=\mathbb{F} H^{*}$ se traduce entonces para $\mathbb{H}$ como $\mathbb{H}^{i j}=\mathbb{H}^{j i}$. 
Observación 2.7. Como es bien sabido, en el caso de sistemas Hamiltonianos simples los puntos críticos adoptan la forma $\bar{\alpha}=0_{\bar{q}} \in T_{\bar{q}}^{*} Q$, con $\mathrm{d} h(\bar{q})=0$ (usando la Observación 2.6 y la Proposición 1.1). Por esta razón, consideraremos solamente este tipo de puntos críticos.

En relación a las incógnitas de (2.10), consideremos ahora que $V$ es también simple [ver (1.40)]. Utilizaremos para $V$ una notación análoga a la usada para $H$. Por ejemplo, escribiremos $V=\mathfrak{v}+v \circ \pi$ y denotaremos por $\mathfrak{b}_{V}$ a la forma bilineal asociada al término cinético de la función $V$, i.e.

$$
\mathfrak{b}_{V}: T^{*} Q \times T^{*} Q \rightarrow \mathbb{R}:(\alpha, \sigma) \longmapsto \frac{1}{2}\left\langle\alpha, \phi^{\sharp}(\sigma)\right\rangle .
$$

En estas condiciones, es claro que $\mathbb{F} V=\mathbb{F} \mathfrak{v}$.

Observación 2.8. En el caso de funciones simples, la función $V$ resultará definida positiva alrededor de $\bar{\alpha}$ si y sólo si el término potencial $v$ es definido positivo alrededor de $\bar{q}$. En particular, esto implica que la función $v$ debe tener un mínimo local aislado en $\bar{q}$, i.e. $\mathrm{d} v(\bar{q})=0$.

Supongamos además que $z_{g}: T^{*} Q \rightarrow T^{*} Q$ está dada por

$$
\left\langle z_{g}(\alpha), w\right\rangle=\mathfrak{Z}_{g}\left(\alpha, \alpha, \mathbb{F}^{-1}(w)\right), \quad \forall q \in Q, \alpha \in T_{q}^{*} Q, w \in T_{q} Q,
$$

para algún campo tensorial $\mathfrak{Z}_{g}: T^{*} Q \times T^{*} Q \times T^{*} Q \rightarrow \mathbb{R}$. Debido a esta elección particular para la aplicación $z_{g}$, ésta resulta cuadrática en $\alpha$. Observemos que el campo tensorial $\mathfrak{Z}_{g}$ puede considerarse simétrico en sus dos primeros argumentos, i.e.

$$
\mathfrak{Z}_{g}\left(\alpha_{1}, \alpha_{2}, \alpha\right)=\mathfrak{Z}_{g}\left(\alpha_{2}, \alpha_{1}, \alpha\right) .
$$

Además, usando la condición giroscópica [ver la Ec. (2.15)], es claro que

$$
\mathfrak{Z}_{g}(\alpha, \alpha, \alpha)=0,
$$

para todo $\alpha \in T_{q}^{*} Q$. Recíprocamente, cualquier tensor cumpliendo (2.21) da origen, via (2.9) y (2.19), a una fuerza giroscópica.

Regresando a las condiciones de matching, dado que los términos cinéticos de $H$ y $V$ son funciones cuadráticas y que sus términos potenciales están definidos por funciones básicas, la siguiente proposición es de fácil demostración.

Proposición 2.2. Suponiendo que $H$ y $V$ son funciones simples y considerando que $z_{g}$ cumple (2.19), las condiciones de matching (2.10) se descomponen en dos ecuaciones:

$$
\langle\mathbb{B} \mathfrak{v}(\alpha), \mathbb{F} \mathfrak{h}(\sigma)\rangle-\langle\mathbb{B} \mathfrak{h}(\alpha), \mathbb{F} \mathfrak{v}(\sigma)\rangle-\mathfrak{Z}_{g}(\alpha, \alpha, \sigma)=0,
$$

conocidas como las condiciones de matching cinéticas, $y$ [ver Ec. (1.17)]

$$
\langle\mathrm{d} v(\pi(\sigma)), \mathbb{F} \mathfrak{h}(\sigma)\rangle-\langle\mathrm{d} h(\pi(\sigma)), \mathbb{F} \mathfrak{v}(\sigma)\rangle=0,
$$

conocidas como las condiciones de matching potenciales. Ambas condiciones deben cumplirse para todo

$$
\alpha \in T^{*} Q \quad y \quad \sigma \in \hat{W}_{\alpha} .
$$


Observación 2.9. Notemos que [ver Ec. (2.11)] $\hat{W}_{\alpha}=\mathbb{F}^{-1}\left(W_{\alpha}^{\circ}\right)$ es el complemento ortogonal de $W_{\alpha}$ con respecto a la forma bilineal $\mathfrak{b}_{V}$ [ver (2.18)]. De hecho, si $\zeta \in W_{\alpha} \mathrm{y} w \in W_{\alpha}^{\circ}$, entonces

$$
\mathfrak{b}_{V}\left(\zeta, \mathbb{F} \mathfrak{v}^{-1}(w)\right)=\left\langle\zeta, \mathbb{F} \mathfrak{v} \circ \mathbb{F} \mathfrak{v}^{-1}(w)\right\rangle=\langle\zeta, w\rangle=0
$$

Tomando una carta coordenada local $(U, \varphi)$ y combinando (1.15), (1.16) y (1.43),

$$
\begin{aligned}
\langle\mathbb{B} \mathfrak{v}(\alpha), \mathbb{F} \mathfrak{h}(\sigma)\rangle-\langle\mathbb{B} \mathfrak{h}(\alpha), \mathbb{F} \mathfrak{v}(\sigma)\rangle & =\left(\frac{\partial \mathbb{V}^{i j}}{\partial q^{k}}(\mathbf{q}) \mathbb{H}^{k l}(\mathbf{q})-\frac{\partial \mathbb{H}^{i j}}{\partial q^{k}}(\mathbf{q}) \mathbb{V}^{k l}(\mathbf{q})\right) p_{i} p_{j} \widetilde{p}_{l} \\
& +2 \Gamma_{k s}^{j}(\mathbf{q})\left(\mathbb{V}^{i s}(\mathbf{q}) \mathbb{H}^{k l}(\mathbf{q})-\mathbb{H}^{i s}(\mathbf{q}) \mathbb{V}^{k l}(\mathbf{q})\right) p_{i} p_{j} \widetilde{p}_{l}(2.26)
\end{aligned}
$$

y

$$
\langle\mathrm{d} v(\pi(\sigma)), \mathbb{F} \mathfrak{h}(\sigma)\rangle-\langle\mathrm{d} h(\pi(\sigma)), \mathbb{F} \mathfrak{v}(\sigma)\rangle=\left(\frac{\partial v}{\partial q^{k}}(\mathbf{q}) \mathbb{H}^{k l}(\mathbf{q})-\frac{\partial h}{\partial q^{k}}(\mathbf{q}) \mathbb{V}^{k l}(\mathbf{q})\right) \widetilde{p}_{l},
$$

con $\alpha=\varphi^{*}(\mathbf{q}, \mathbf{p})$ y $\sigma=\varphi^{*}(\mathbf{q}, \widetilde{\mathbf{p}})$. En consecuencia, las Ecs. (2.22) y (2.23) se transforman en

$$
\begin{aligned}
& \left(\frac{\partial \mathbb{V}^{i j}(\mathbf{q})}{\partial q^{k}} \mathbb{H}^{k l}(\mathbf{q})-\frac{\partial \mathbb{H}^{i j}(\mathbf{q})}{\partial q^{k}} \mathbb{V}^{k l}(\mathbf{q})-2 \mathfrak{Z}_{g}^{i j l}(\mathbf{q})\right) p_{i} p_{j} \widetilde{p}_{l} \\
& \quad+2 \Gamma_{k s}^{j}(\mathbf{q})\left(\mathbb{V}^{i s}(\mathbf{q}) \mathbb{H}^{k l}(\mathbf{q})-\mathbb{H}^{i s}(\mathbf{q}) \mathbb{V}^{k l}(\mathbf{q})\right) p_{i} p_{j} \widetilde{p}_{l}=0
\end{aligned}
$$

y

$$
\left(\frac{\partial v}{\partial q^{a}}(\mathbf{q}) \mathbb{H}^{a k}(\mathbf{q})-\frac{\partial h}{\partial q^{a}}(\mathbf{q}) \mathbb{V}^{a k}(\mathbf{q})\right) \widetilde{p}_{k}=0
$$

para todo $\mathbf{q} \in \varphi(U), \mathbf{p} \in\left(\varphi_{\mathbf{q}}^{*}\right)^{-1}\left(T_{q}^{*} Q\right)$ y $\widetilde{\mathbf{p}} \in\left(\varphi_{\mathbf{q}}^{*}\right)^{-1}\left(\hat{W}_{\varphi_{\mathbf{q}}^{*}(\mathbf{p})}\right)$. (Aquí, los números $\mathfrak{Z}_{g}^{i j k}(\mathbf{q})$ son lo coeficientes de la representación matricial coordenada de $\mathfrak{Z}_{g}$ en $q$.)

Observemos que, tomando en cuenta todas las suposiciones hechas hasta aquí [ver Ec. (2.14)]

$$
\begin{aligned}
y(\alpha) & :=z_{d}(\alpha)+\mathfrak{Z}_{g}\left(\alpha, \alpha, \mathbb{F v}^{-1}(\cdot)\right)-\mathbb{F} \mathfrak{v}^{-1} \circ(\mathbb{F} \mathfrak{h} \circ \mathbb{B} \mathfrak{v}(\alpha)-\mathbb{F} \mathfrak{v} \circ \mathbb{B h}(\alpha)) \\
& -\mathbb{F} \mathfrak{v}^{-1} \circ(\mathbb{F} \mathfrak{h} \circ \mathrm{d} v(\pi(\alpha))-\mathbb{F} \mathfrak{v} \circ \mathrm{d} h(\pi(\alpha))),
\end{aligned}
$$

con $z_{d}$ cumpliendo las ecuaciones algebraicas (2.16) y (2.17).

Observación 2.10. Si $\bar{\alpha}=0_{\bar{q}}$ es un punto crítico de $X_{H}$, y dado que

$$
\mathbb{B} \mathfrak{v}(0)=\mathbb{B h}(0)=0 \quad \text { y } \quad \mathfrak{Z}_{g}\left(0,0, \mathbb{F}^{-1}(\cdot)\right)=0
$$

la condición (2.17) para $z_{d}$ se reduce a $z_{d}(\bar{\alpha})=\mathbb{F}^{-1} \circ \mathbb{F h}(\mathrm{d} v(\bar{q}))=0$. Esto se deduce de las Observaciones 2.6 y 2.8 .

Tomando en cuenta esta última observación y el hecho de que

$$
\left\langle z_{d}(\alpha), \mathbb{F} V(\alpha)\right\rangle=\left\langle z_{d}(\alpha), \mathbb{F} \mathfrak{v}(\alpha)\right\rangle=\mathfrak{b}_{V}\left(z_{d}(\alpha), \alpha\right),
$$


las Ecs. (2.16) y (2.17) puede reescribirse como

$$
\mathfrak{b}_{V}\left(z_{d}(\alpha), \alpha\right) \leq 0, \quad z_{d}(\alpha) \in W_{\alpha} \quad \mathrm{y} \quad z_{d}(\bar{\alpha})=0
$$

Las Ecuaciones (2.22), (2.23) y (2.24) definen una versión generalizada del método IDA-PBC tradicional [35], también conocido como el método energy plus force shaping. En la siguiente subsección consideraremos dos suposiciones adicionales que nos conducirán a un nuevo conjunto de condiciones de matching, originalmente ${ }^{4}$ estudiadas por Chang en $[14,15]$ (ver también $[12,13]$ para la contraparte Lagrangiana).

\subsubsection{Condiciones de matching simples}

En primer lugar, supongamos que existe un subfibrado $W \subset T^{*} Q$ tal que [recordar la Ec. (2.8)]

$$
\mathcal{W}_{\alpha}=\operatorname{vlift}_{\alpha}\left(W_{\pi(\alpha)}\right), \quad \forall \alpha \in T^{*} Q
$$

Siguiendo (2.11), a partir de $W$ definamos un nuevo subfibrado de $T^{*} Q$,

$$
\hat{W}:=(\mathbb{F} \mathfrak{v}(W))^{\circ}=\mathbb{F}^{-1}\left(W^{\circ}\right) .
$$

Observación 2.11. Notemos que, de acuerdo a la Observación 2.9, $\hat{W}$ es el complemento ortogonal de $W$ con respecto al tensor $\mathfrak{b}_{V}$. En particular, tenemos que $T^{*} Q=W \oplus \hat{W}$.

Bajo estas suposiciones, Chang mostró que existe una solución $\left(\mathfrak{v}, \mathfrak{Z}_{g}\right)$ de (2.22) si y sólo si v satisface

$$
\langle\mathbb{B} \mathfrak{v}(\sigma), \mathbb{F} \mathfrak{h}(\sigma)\rangle-\langle\mathbb{B h}(\sigma), \mathbb{F} \mathfrak{v}(\sigma)\rangle=0, \quad \forall \sigma \in \hat{W}
$$

Más aun, es posible mostrar, usando álgebra lineal elemental, que $\left(\mathfrak{v}, \mathfrak{Z}_{g}\right)$ es una solución de (2.22) si y sólo si v satisface la ecuación de arriba y $\mathfrak{Z}_{g}$ se obtiene siguiendo estos pasos:

1. definamos $\Upsilon: T^{*} Q \times T^{*} Q \times T^{*} Q \rightarrow \mathbb{R}$ como

$$
\Upsilon\left(\alpha_{1}, \alpha_{2}, \alpha_{3}\right):=\left\langle\mathbb{B} \mathfrak{b}_{V}\left(\alpha_{1}, \alpha_{2}\right), \mathbb{F} \mathfrak{h}\left(\alpha_{3}\right)\right\rangle-\left\langle\mathbb{B} \mathfrak{b}_{H}\left(\alpha_{1}, \alpha_{2}\right), \mathbb{F} \mathfrak{v}\left(\alpha_{3}\right)\right\rangle ;
$$

2. fijemos un tensor $A: W \times W \times \hat{W} \rightarrow \mathbb{R}$ cumpliendo

$$
A\left(\alpha_{1}, \alpha_{2}, \alpha\right)=-A\left(\alpha_{2}, \alpha_{1}, \alpha\right), \quad \forall \alpha_{1}, \alpha_{2} \in W, \alpha \in \hat{W}
$$

y un tensor $B: W \times W \times W \rightarrow \mathbb{R}$ cumpliendo (2.20) y (2.21) sobre $W$; y finalmente

\footnotetext{
${ }^{4}$ De hecho, tales condiciones de matching aparecieron por primera vez [25], aunque sin una derivación.
} 
3. definamos $\mathfrak{Z}_{g}: T^{*} Q \times T^{*} Q \times T^{*} Q \rightarrow \mathbb{R}$ como $^{5}$

$$
\left\{\begin{array}{l}
\mathfrak{Z}_{g}\left(\alpha_{1}, \alpha_{2}, \sigma\right):=\Upsilon\left(\alpha_{1}, \alpha_{2}, \sigma\right) \\
\mathfrak{Z}_{g}\left(\sigma_{1}, \sigma_{2}, \gamma\right):=-\Upsilon\left(\gamma, \sigma_{2}, \sigma_{1}\right)-\Upsilon\left(\gamma, \sigma_{1}, \sigma_{2}\right) \\
\mathfrak{Z}_{g}\left(\gamma_{1}, \sigma, \gamma_{2}\right):=\mathfrak{Z}_{g}\left(\sigma, \gamma_{1}, \gamma_{2}\right):=-\frac{1}{2}\left[\Upsilon\left(\gamma_{1}, \gamma_{2}, \sigma\right)+A\left(\gamma_{1}, \gamma_{2}, \sigma\right)\right] \\
\mathfrak{Z}_{g}\left(\gamma_{1}, \gamma_{2}, \gamma_{3}\right):=B\left(\gamma_{1}, \gamma_{2}, \gamma_{3}\right)
\end{array}\right.
$$

$\operatorname{con} \alpha_{i} \in T^{*} Q, \sigma, \sigma_{i} \in \hat{W}$ y $\gamma, \gamma_{i} \in W$ para $i=1,2,3$.

Luego, existe una solución $\left(\mathfrak{v}, v, \mathfrak{Z}_{g}\right)$ de (2.22), (2.23) y (2.24) si y sólo si las ecuaciones

$$
\begin{aligned}
\langle\mathbb{B} \mathfrak{v}(\sigma), \mathbb{F} \mathfrak{h}(\sigma)\rangle-\langle\mathbb{B h}(\sigma), \mathbb{F} \mathfrak{v}(\sigma)\rangle & =0, \\
\langle\mathrm{~d} v(\pi(\sigma)), \mathbb{F} \mathfrak{h}(\sigma)\rangle-\langle\mathrm{d} h(\pi(\sigma)), \mathbb{F} \mathfrak{v}(\sigma)\rangle & =0, \quad \forall \sigma \in \hat{W},
\end{aligned}
$$

tienen solución para $\mathfrak{v}$ y $v$ únicamente. Éstas son las nuevas condiciones de matching que mencionamos al final de la sección anterior, que llamaremos condiciones de matching simples. Tomando coordenadas locales, tales condiciones se escriben

$$
\left(\frac{\partial \mathbb{V}^{i j}}{\partial q^{k}}(\mathbf{q}) \mathbb{H}^{k l}(\mathbf{q})-\frac{\partial \mathbb{H}^{i j}}{\partial q^{k}}(\mathbf{q}) \mathbb{V}^{k l}(\mathbf{q})\right) p_{i} p_{j} p_{l}=0
$$

y

$$
\left(\frac{\partial v}{\partial q^{k}}(\mathbf{q}) \mathbb{H}^{k l}(\mathbf{q})-\frac{\partial h}{\partial q^{k}}(\mathbf{q}) \mathbb{V}^{k l}(\mathbf{q})\right) p_{l}=0
$$

para todo $\mathbf{q} \in \varphi(U)$ y $\mathbf{p} \in\left(\varphi_{\mathbf{q}}^{*}\right)^{-1}\left(\hat{W}_{\varphi^{-1}(\mathbf{q})}\right)$, o equivalentemente $[\operatorname{ver}(2.32)]\left(\varphi^{-1}\right)_{*, \mathbf{q}}(\mathbb{V}(\mathbf{q}) \cdot \mathbf{p}) \in$ $W_{\varphi^{-1}(\mathbf{q})}^{\circ}$

Observación 2.12. Estas ecuaciones son (a menos de un signo) las Ecs. 2.21 y 2.29 de [15] para

$$
M^{i j}=\mathbb{H}^{i j}, \quad \widehat{M}^{i j}=\mathbb{V}^{i j}, \quad V=h, \quad \widehat{V}=v, \quad \text { y } \quad G^{\perp}=W^{\circ} .
$$

Retomemos ahora las ecuaciones algebraicas (2.30). Para empezar, notemos que podemos reemplazar los espacios $W_{\alpha}$ por el subfibrado vectorial $W$. Supongamos que tenemos una solución $z_{d}$ de (2.30), y consideremos la proyección ortogonal $P$ con imagen $W$ (ver la Observación 2.11). Luego,

$$
\mathfrak{b}_{V}\left(z_{d}(\alpha), \alpha\right)=\mathfrak{b}_{V}\left(z_{d}(\alpha), P(\alpha)\right)=-\mu(\alpha)
$$

para alguna función no negativa $\mu: T^{*} Q \rightarrow \mathbb{R}$ tal que $\mu(\sigma)=0$ para todo $\sigma \in \hat{W}$. Fijando $k \in \mathbb{R}$, es claro que

$$
x(\alpha):=z_{d}(\alpha)+k P(\alpha) \in W
$$

\footnotetext{
${ }^{5}$ Chang describió una construcción similar para mostrar la existencia de soluciones de (2.22) (ver las Ecs. 2.8 a 2.11 en la Ref. [15], y reemplazar $\Upsilon$ y $\mathfrak{Z}_{g}$ por $S$ y $C$, respectivamente), pero tomando $A=0$ y $B=0$.
} 
define un morfismo de fibrados, cuya imagen está contenida en $W$. Además, usando esta última ecuación

$$
\left.\mathfrak{b}_{V}(x(\alpha), P(\alpha))=\mathfrak{b}_{V}\left(z_{d}(\alpha), P(\alpha)\right)+k \mathfrak{b}_{V}(P(\alpha)), P(\alpha)\right),
$$

de modo que, para todo $\alpha \notin \hat{W}$

$$
k=\frac{\mathfrak{b}_{V}(x(\alpha), P(\alpha))+\mu(\alpha)}{\mathfrak{b}_{V}(P(\alpha), P(\alpha))} .
$$

En otras palabras, existe un morfismo de fibrados $x$, cuya imagen está contenida en $W$ y para la cual

$$
z_{d}(\alpha)=x(\alpha)-\frac{\mathfrak{b}_{V}(x(\alpha), P(\alpha))+\mu(\alpha)}{\mathfrak{b}_{V}(P(\alpha), P(\alpha))} P(\alpha), \quad \forall \alpha \notin \hat{W}
$$

Dado que $T^{*} Q \backslash \hat{W}$ es una subvariedad abierta y densa en $T^{*} Q$, esto dice que la solución $z_{d}$ puede calcularse sobre $T^{*} Q \backslash \hat{W}$ usando la expresión (2.39) y sobre el resto de $T^{*} Q$ por continuidad. En particular, tenemos que

$$
\lim _{\alpha \rightarrow \bar{\alpha}}\left(x(\alpha)-\frac{\mathfrak{b}_{V}(x(\alpha), P(\alpha))+\mu(\alpha)}{\mathfrak{b}_{V}(P(\alpha), P(\alpha))} P(\alpha)\right)=0,
$$

puesto que $z_{d}(\bar{\alpha})=0$.Recíprocamente, si:

i. fijamos una función no negativa $\mu: T^{*} Q \rightarrow \mathbb{R}$ tal que $\mu(\sigma)=0$ para todo $\sigma \in \hat{W}$;

ii. fijamos un morfismo de fibrados $x: T^{*} Q \rightarrow T^{*} Q$ con imagen contenida en $W$ y tal que la fórmula (2.39) defina una aplicación suave en todo $T^{*} Q$ y cumpliendo (2.40);

iii. definimos $z_{d}: T^{*} Q \rightarrow T^{*} Q$ mediante la fórmula (2.39);

entonces tenemos una solución de (2.30). Por ejemplo, podemos tomar

$$
\mu(\alpha):=\mathfrak{b}_{V}(P(\alpha), P(\alpha)) \quad \text { y } \quad x(\alpha):=-\mu(\alpha) \xi(\pi(\alpha))
$$

siendo $\xi: Q \rightarrow T^{*} Q$ cualquier 1-forma sobre $Q$ con imagen contenida en $W$ (esto siempre es posible, puesto que $W$ es un subfibrado vectorial).

En conclusión, tenemos un nuevo método para resolver el problema $\mathbf{P}$. Suponiendo que hemos fijado una conexión sobre $T^{*} Q$.

Definición 2.4. Dado un sistema subactuado $(H, \mathcal{W})$, con $H=\mathfrak{h}+h \circ \pi$ simple y $\mathcal{W}$ definido por un subfibrado $W$ [ver Ec. (2.31)], y dado un punto crítico $\bar{\alpha} \in T^{*} Q$ de $X_{H}$, el método $\mathbf{C H}$ simple consiste en:

- encontrar una solución $V=\mathfrak{v}+v \circ \pi$ de las Ecs. (2.35) y (2.36), con $v$ definida positiva alrededor de $\bar{\alpha}$;

- fijar un tensor $\mathfrak{Z}_{g}: T^{*} Q \times T^{*} Q \times T^{*} Q \rightarrow \mathbb{R}$ mediante los pasos 1 a 3 de arriba [ver Ec. (2.34)]; 
- fijar un morfismo de fibrados $z_{d}: T^{*} Q \rightarrow T^{*} Q$ mediante los pasos i aiii de arriba [ver Ec. (2.39)]; y

- definir un morfismo de fibrados $y: T^{*} Q \rightarrow T^{*} Q$ como en (2.29) e $Y \in \mathfrak{X}\left(T^{*} Q\right)$ como el levantamiento vertical de $y$.

Es claro que el método $\mathrm{CH}$ simple es un método de estabilización de Lyapunov según la Definición 2.1. En particular, $V$ e $Y$ satisfacen (2.3) y es sencillo mostrar que el método $\mathrm{CH}$ simple está contenido (en el sentido de la Definición 2.2) en el método CH general (ver Definición 2.3).

Volviendo ahora a las nuevas condiciones de matching (2.35) y (2.36) [junto con sus versiones locales (2.37) y (2.38)], la mejora desarrollada por Chang en cuanto a la simplificación de las Ecs. (2.22) y (2.23) es doble. Por un lado, las tres incógnitas $\mathfrak{v}, v$ y $\mathfrak{Z}_{g}$ en (2.22) y (2.23) están ahora desacopladas. ${ }^{6}$ Por otro lado, la Ecuación (2.28) [la versión local de (2.22)] ha sido reemplazada por (2.35), que es un conjunto más sencillo de ecuaciones diferenciales, no sólo porque su escritura es más simple sino también porque contiene una cantidad menor de ecuaciones. En efecto, puede mostrarse que el número de ecuaciones en (2.28) y (2.37) es, respectivamente,

$$
\frac{n(n+1)(n-m)}{2}
$$

y

$$
\frac{(n-m+2)(n-m+1)(n-m)}{6},
$$

$\operatorname{con} n=\operatorname{dim} Q$ y $m=\operatorname{dim} W$.

Observación 2.13. En la Referencia [16] se prueba que, incluso en el método IDA-PBC tradicional (donde las incógnitas $\mathfrak{Z}_{g}^{i j k}$ adoptan una forma particular), la cantidad de ecuaciones en (2.28) pueden reducirse de (2.41) a (2.42) utilizando la libertad que existe en la elección de las incógnitas $\mathfrak{Z}_{g}^{i j k}$. Por lo tanto, la contribución principal de Chang fue quizá, no la reducción del número de ecuaciones, sino más bien precisar una manera útil de lograr esa reducción.

Las Ecuaciones (2.37) y (2.38) [y por lo tanto (2.35) y (2.36)] ya aparecieron en el Capítulo 1 [ver (1.44) y (1.45)] en el contexto del método LCB simple para sistemas subactuados con un único actuador. En la última sección de este capítulo, veremos que las mismas ecuaciones pueden obtenerse, en el contexto del LCB, para sistemas con número de actuadores arbitrario, extendiendo de esta manera el método LCB simple a sistemas con grado de actuación arbitrario. Más aun, probaremos que la versión del método $\mathrm{CH}$ desarrollada por Chang es exactamente el método LCB simple.

\footnotetext{
${ }^{6}$ Más precisamente, podemos encontrar $\mathfrak{v}$ resolviendo (2.35), luego, para tal solución $\mathfrak{v}$, podemos hallar $V$ resolviendo (2.36), y por último, usando $\mathfrak{v}$ y $V$, podemos construir $\mathfrak{Z}_{g}$ siguiendo los pasos del 1 al 3 listados arriba.
} 


\subsection{El método LCB revisitado: maximalidad del LCB}

Siguiendo la Definición 2.1, es claro que el método LCB (ver Def. 1.9) es un método de estabilización de Lyapunov. Vale notar que este método está definido, esencialmente, por la Ec. (1.36) y por lo tanto podemos identificarlo con esa ecuación. Dado que $\mathcal{W}$ es un subfibrado vertical e $Y$ es un campo vectorial vertical, podemos escribir (ver Observación 1.11) $\mathcal{W}_{\alpha}=\operatorname{vlift}_{\alpha}\left(W_{\alpha}\right)$ e $Y(\alpha)=\operatorname{vlift}_{\alpha}(y(\alpha)$ ), para un único subespacio $W_{\alpha} \subset T_{\pi(\alpha)}^{*} Q$ y un único morfismo de fibrados $y: T^{*} Q \rightarrow T^{*} Q$. Con esta notación y según la Proposición 1.2,

$$
\langle\mathrm{d} V(\alpha), Y(\alpha)\rangle=\langle y(\alpha), \mathbb{F} V(\alpha)\rangle
$$

Además, las condiciones $Y(\bar{\alpha})=0$ y $Y(\alpha) \in \mathcal{W}_{\alpha}$ se traducen a $y(\bar{\alpha})=0$ e $y(\alpha) \in W_{\alpha}$, respectivamente. Por otro lado

$$
\left\langle\mathrm{d} V(\alpha), X_{H}(\alpha)\right\rangle=\{V, H\}(\alpha),
$$

siendo $\{V, H\}$ el corchete de Poisson canónico entre $V$ y $H$. Combinando todo esto, podemos escribir (1.36) como

$$
\langle y(\alpha), \mathbb{F} V(\alpha)\rangle=-\mu(\alpha)-\{V, H\}(\alpha) \quad \text { e } \quad y(\alpha) \in W
$$

Es importante mencionar que cualquier método de estabilización para $(H, \mathcal{W})$ que dé origen a una ley de control $Y \subset \mathcal{W}$ y a una función de Lyapunov $V$ para el campo $X_{H}+Y$ (que define el sistema a lazo cerrado relacionado) y un punto crítico de $X_{H}$, como es el caso de todas las versiones del energy shaping, puede ser reducido al método LCB, i.e. a resolver la Ec. (1.36) [o, de manera equivalente, a resolver la Ec. (2.43)]. Más precisamente,

Teorema 2.1. Sean $(H, \mathcal{W})$ un sistema subactuado y $\bar{\alpha} \in T^{*} Q$ un punto crítico de $X_{H}$. Si $Y$ es un campo vectorial con valores en $\mathcal{W} y V$ es una función de Lyapunov para $X_{H}+Y$ y $\bar{\alpha}$, entonces $V$ es definida positiva alrededor de $\bar{\alpha}, \mu:=-\mathfrak{i}_{X_{H}+Y} \mathrm{~d} V$ es no negativa, $Y(\bar{\alpha})=0$ y todas ellas satisfacen la Ec. (1.36). En particular, la señal de control $Y$ está dada por el método LCB (ver Definición 1.9).

Demostración. Dado un campo $Y \subset \mathcal{W}$, si $V$ es una función de Lyapunov para $X:=X_{H}+Y$ y $\bar{\alpha}$, el resultado se sigue fácilmente del hecho de que $\bar{\alpha}$ debe ser un punto crítico de $X$, los ítems L1 y L2 (ver Teorema 1.1), y la Ec. (1.37).

En términos de las Definiciones 2.1 y 2.2, este teorema dice que el método LCB contiene a todos los métodos de estabilización de Lyapunov, i.e. es un elemento maximal entre tales métodos. En otras palabras, el método LBC es el método de estabilización más general entre los métodos de estabilización de Lyapunov (ver la Observación 2.2). En particular, toda versión del método CH (ver Definición 2.3) está contenido en el método LCB. 


\subsection{El método LCB para funciones simples}

En la Ref. [22] se realizó un estudio en profundidad de la Ec. (2.43) para sistemas subactuados con un único actuador. En esta sección extenderemos ese estudio para incluir sistemas subactuados con un número arbitrario de actuadores. Más precisamente, dado un sistema subactuado $(H, \mathcal{W})$ y funciones no negativas $V$ y $\mu$, estudiaremos bajo qué condiciones existe un morfismo de fibrados y que resuelva (2.43) (considerando a $V$ y $\mu$ como datos en (2.43), en lugar de incógnitas). Nos concentraremos en particular en el caso en que tanto $H$ como $V$ son funciones simples. En este caso, mostraremos que el problema de existencia está relacionado con un conjunto de PDEs para $V$, que definen el método LCB simple tal y como apareció en la Ref. [22]. Veremos que estas ecuaciones son exactamente las condiciones de matching (2.35) y (2.36) obtenidas por Chang en $[14,15]$ (relacionadas con el método CH simple de la Definición 2.4). Finalmente, mostraremos que el método LCB y el CH en sus versiones simples son métodos de estabilización equivalentes.

\subsubsection{Las ecuaciones cinéticas y potenciales}

Siguiendo la notación de la Sección \$2.2.3, consideremos dos funciones simples $H=\mathfrak{h}+h \circ \pi$ and $V=\mathfrak{v}+v \circ \pi$ sobre $T^{*} Q$. Las formas bilineales asociadas a $\mathfrak{h}$ y $\mathfrak{v}$ serán denotadas por $\mathfrak{b}_{H}$ y $\mathfrak{b}_{V}$, respectivamente.

Dada una conexión sin torsión sobre $T^{*} Q$, el corchete de Poisson canónico entre $H$ y $V$ puede escribirse, para todo $\alpha \in T^{*} Q$,

$$
\{V, H\}(\alpha)=\langle\mathbb{B} \mathfrak{v}(\alpha), \mathbb{F} \mathfrak{h}(\alpha)\rangle-\langle\mathbb{B h}(\alpha), \mathbb{F} \mathfrak{v}(\alpha)\rangle+\langle\mathrm{d} v(q), \mathbb{F} \mathfrak{h}(\alpha)\rangle-\langle\mathrm{d} h(q), \mathbb{F} \mathfrak{v}(\alpha)\rangle,
$$

con $q=\pi(\alpha)$. Esto es una consecuencia directa de las Ecuaciones (1.17) y (1.21). Tomando una carta coordenada $(U, \varphi)$ y usando las expresiones (2.26) y (2.27),

$$
\begin{aligned}
\{V, H\}(\mathbf{q}, \mathbf{p}) & =\left(\frac{\partial \mathbb{V}^{i j}}{\partial q^{k}}(\mathbf{q}) \mathbb{H}^{k l}(\mathbf{q})-\frac{\partial \mathbb{H}^{i j}}{\partial q^{k}}(\mathbf{q}) \mathbb{V}^{k l}(\mathbf{q})\right) p_{i} p_{j} p_{l} \\
& +\left(\frac{\partial v}{\partial q^{k}}(\mathbf{q}) \mathbb{H}^{k l}(\mathbf{q})-\frac{\partial h}{\partial q^{k}}(\mathbf{q}) \mathbb{V}^{k l}(\mathbf{q})\right) p_{l} .
\end{aligned}
$$

Analizando cuidadosamente esta última expresión, el siguiente resultado se obtiene fácilmente.

Lema 2.1. Si $H y V$ son funciones simples sobre $T^{*} Q$, entonces $\{V, H\}$ es, en cada fibra $T_{q}^{*} Q$, una función impar, i.e.

$$
\{V, H\}(-\alpha)=-\{V, H\}(\alpha) \quad \text { para todo } \alpha \in T^{*} Q
$$

Fijemos ahora un sistema subactuado $(H, \mathcal{W})$ con $H$ simple y $\mathcal{W}$ dado por un subfibrado $W \subset T^{*} Q$ [ver (2.31)]. 
Proposición 2.3. Sea $V: T^{*} Q \rightarrow \mathbb{R}$ una función simple y $\mu: T^{*} Q \rightarrow \mathbb{R}$ una función no negativa. Si existe una solución y: $T^{*} Q \rightarrow T^{*} Q$ de Ec. (2.43) para $V y \mu$, entonces

$$
\{V, H\}(\sigma)=0, \quad \forall \sigma \in \hat{W}
$$

donde $\hat{W}:=(\mathbb{F} \mathfrak{v}(W))^{\circ}=\mathbb{F} \mathfrak{v}^{-1}\left(W^{\circ}\right)$.

Demostración. Por hipótesis [ver Ec. (2.43)]

$$
\langle\alpha, \mathbb{F} \mathfrak{v}(y(\alpha))\rangle=-\mu(\alpha)-\{V, H\}(\alpha) \quad \text { y } \quad y(\alpha) \in W .
$$

Es claro que $\langle\sigma, \mathbb{F} \mathfrak{v}(y(\sigma))\rangle=0$ para todo $\sigma \in \hat{W}$, y en consecuencia

$$
\mu(\sigma)+\{V, H\}(\sigma)=0, \quad \forall \sigma \in \hat{W} .
$$

Supongamos que existe $\sigma_{0} \in \hat{W}$ para el cual $\{V, H\}\left(\sigma_{0}\right) \neq 0$. Si $\{V, H\}\left(\sigma_{0}\right)>0$, entonces $\mu\left(\sigma_{0}\right)<0$. Pero esto no es posible, y entonces $\{V, H\}\left(\sigma_{0}\right)<0$. De acuerdo con la Ec. (2.45),

$$
\{V, H\}\left(-\sigma_{0}\right)=-\{V, H\}\left(\sigma_{0}\right)>0 \text {. }
$$

Dado que $-\sigma_{0} \in \hat{W}$, concluímos que $\mu\left(-\sigma_{0}\right)+\{V, H\}\left(-\sigma_{0}\right)=0$, por lo que $\mu\left(-\sigma_{0}\right)<0$. En consecuencia, $\{V, H\}(\sigma)=0$ para todo $\sigma \in \hat{W}$.

Veamos ahora que es posible escribir (2.46) de otra manera equivalente.

Lema 2.2. Si $H$ y $V$ son funciones simples sobre $T^{*} Q$ y $D \subset T^{*} Q$ es un subfibrado vectorial, las siguientes condiciones son equivalentes.

1. $\{V, H\}(\sigma)=0, \forall \sigma \in D$.

2. Dada una conexión afin sobre $T^{*} Q$, para todo $q \in Q y \sigma \in D_{q}$, las Ecs. (2.35) y (2.36) se cumplen, con $D$ en lugar de $\hat{W}$.

3. Dada una carta coordenada $(U, \varphi)$, para todo $\mathbf{q} \in \varphi(U) y \mathbf{p} \in\left(\varphi_{\mathbf{q}}^{*}\right)^{-1}\left(D_{\varphi^{-1}(\mathbf{q})}\right)$, las Ecs. (2.37) $y$ (2.38) se cumplen.

Demostración. La equivalencia entre los puntos 2 y 3 fue establecida en las Ecuaciones (2.26) y (2.27). Solamente resta probar que 1 implica 3 (su recíproco es inmediato). Si $\{V, H\}(\sigma)=0, \forall \sigma \in D$, se sigue de la Ecuación (2.44) que, tomando una carta coordenada $(U, \varphi)$,

$$
\left(\frac{\partial \mathbb{V}^{i j}}{\partial q^{k}}(\mathbf{q}) \mathbb{H}^{k l}(\mathbf{q})-\frac{\partial \mathbb{H}^{i j}}{\partial q^{k}}(\mathbf{q}) \mathbb{V}^{k l}(\mathbf{q})\right) p_{i} p_{j} p_{l}+\left(\frac{\partial v}{\partial q^{k}}(\mathbf{q}) \mathbb{H}^{k l}(\mathbf{q})-\frac{\partial h}{\partial q^{k}}(\mathbf{q}) \mathbb{V}^{k l}(\mathbf{q})\right) p_{l}=0,
$$


para todo $\mathbf{q} \in \varphi(U)$ y $\mathbf{p} \in\left(\varphi_{\mathbf{q}}^{*}\right)^{-1}\left(V_{\varphi^{-1}(\mathbf{q})}\right)$. Tenemos que mostrar que cada uno de los términos entre paréntesis se anula. Fijando un $\widehat{\mathbf{p}} \in\left(\varphi_{\mathbf{q}}^{*}\right)^{-1}\left(D_{\varphi^{-1}(\mathbf{q})}\right)$, definamos

$$
A:=\left(\frac{\partial \mathbb{V}^{i j}}{\partial q^{k}}(\mathbf{q}) \mathbb{H}^{k l}(\mathbf{q})-\frac{\partial \mathbb{H}^{i j}}{\partial q^{k}}(\mathbf{q}) \mathbb{V}^{k l}(\mathbf{q})\right) \widehat{p}_{i} \widehat{p}_{j} \widehat{p}_{l}
$$

y

$$
B:=\left(\frac{\partial v}{\partial q^{k}}(\mathbf{q}) \mathbb{H}^{k l}(\mathbf{q})-\frac{\partial h}{\partial q^{k}}(\mathbf{q}) \mathbb{V}^{k l}(\mathbf{q})\right) \widehat{p}_{l}
$$

Luego $A \lambda^{3}+B \lambda=0$ para todo $\lambda \in \mathbb{R}$ (pues $\left(\varphi_{\mathbf{q}}^{*}\right)^{-1}\left(D_{\varphi^{-1}(\mathbf{q})}\right)$ es un espacio vectorial). Pero esto es posible únicamente si $A=B=0$.

Combinando este último Lema junto con la Proposición 2.3, tenemos el siguiente resultado.

Proposición 2.4. Consideremos una función simple $V: T^{*} Q \rightarrow \mathbb{R}$ y una función no negativa $\mu: T^{*} Q \rightarrow \mathbb{R}$. Si existe una solución y: $T^{*} Q \rightarrow T^{*} Q$ de la Ec. (2.43) para $V y \mu$, entonces, para cada conexión afin sobre $T^{*} Q$,

$$
\begin{aligned}
\langle\mathbb{B} \mathfrak{v}(\sigma), \mathbb{F} \mathfrak{h}(\sigma)\rangle-\langle\mathbb{B} \mathfrak{h}(\sigma), \mathbb{F} \mathfrak{v}(\sigma)\rangle & =0, \\
\langle\mathrm{~d} v(\pi(\sigma)), \mathbb{F} \mathfrak{h}(\sigma)\rangle-\langle\mathrm{d} h(\pi(\sigma)), \mathbb{F} \mathfrak{v}(\sigma)\rangle & =0, \quad \forall \sigma \in \hat{W} .
\end{aligned}
$$

Notemos que las Ecuaciones (2.47) y (2.48) son exactamente las condiciones de matching simples (2.35) y (2.36) deducidas por Chang.

Observación 2.14. Esto quiere decir, usando nuevamente el Lema 2.2, que las condiciones de matching simples pueden escribirse como en (2.46), o equivalentemente

$$
\{V, H\} \circ \mathbb{F} V^{-1}(w)=0, \quad \forall w \in W^{\circ},
$$

que es una expresión independiente de coordenadas e independiente de la conexión escogida.

Las Proposiciones 2.3 y 2.4 establecen que la Ecuación (2.46), o equivalentemente las Ecuaciones (2.47) y (2.48), que tiene una función de la forma $V=\mathfrak{v}+v \circ \pi$ como incógnita, definen una condición necesaria para la existencia de soluciones de (1.36). Veamos que las mismas también son suficientes. En la Sección \$2.2.4 vimos que si $V$ es simple y satisface (2.47) y (2.48), entonces existe un campo vectorial $Y \subset \mathcal{W}$ cumpliendo (2.3). De hecho, $Y$ puede construirse siguiendo el procedimiento descripto en la Definición 2.4. Por lo tanto, $Y$ satisface la Ec. (1.36) para $V$ y la función (no negativa) $\mu:=-\mathfrak{i}_{X_{H}+Y} \mathrm{~d} V$. En conclusión,

Teorema 2.2. Dada una función simple $V: T^{*} Q \rightarrow \mathbb{R}$, existen una solución y de la Ec. (2.43) para $V$ y una función no negativa $\mu$ si y sólo si $V$ es solución de (2.46).

Observación 2.15. A diferencia de la situación considerada en la Referencia [22], dado que en nuestro caso el subfibrado $W$ tiene dimensión arbitraria (i.e. no necesariamente 1 ), no podemos asegurar que la solución $y$ de la Ec. (2.43) sea única (para $V$ y $\mu$ fijas). En el caso en que $\operatorname{dim} W_{q}=\operatorname{span}\{\xi\}$ para todo 
$q \in Q$ y escribiendo $y(\alpha)=\lambda(\alpha) \xi(\pi(\alpha))$, vimos en el Capítulo 1 [ver Ec. (1.38)] que la función $\lambda$ está dada necesariamente por

$$
\lambda(\alpha):=-\frac{\mu(\alpha)+\{V, H\}(\alpha)}{\langle\xi(\pi(\alpha)), \mathbb{F} V(\alpha)\rangle}
$$

y por lo tanto la señal $y$ es única.

Tomando en cuenta el carácter maximal del método LCB (ver Teorema 2.1), este último teorema dice que (2.46), y en consecuencia las condiciones de matching simples, son un ingrediente clave que debe estar presente (explícitamente o no) en cualquier método de estabilización que involucre la construcción de una función de Lyapunov simple. Más precisamente, si construimos una señal de control $Y$ y una función de Lyapunov $V$ para $X_{H}+Y$ por medio de un método de estabilización, entonces $V$ debe satisfacer la Ec. (2.46).

\subsubsection{El método LCB simple}

Veamos de qué manera podemos extender la Definición 1.10 para incluir espacios $W_{\alpha}$ definidos a partir de una codistribución de dimensión arbitraria (finita). Fijemos un sistema subactuado $(H, \mathcal{W})$ con $H$ simple y $\mathcal{W}$ definida por un subfibrado $W \subset T^{*} Q$. Fijemos además un punto crítico $\bar{\alpha}=(\bar{q}, 0)$ de $X_{H}$ (ver Observación 2.7). Supongamos que $V$ es una función simple, $\mu$ una función no negativa e $y: T^{*} Q \rightarrow T^{*} Q$ un morfismo de fibrados tales que $y(\bar{\alpha})=0$ y que cumplen la Ec. (2.43). Es claro que $y$ puede reescribirse como

$$
y(\alpha):=z(\alpha)-\mathbb{F} V^{-1} \circ(\mathbb{F} H \circ \mathbb{B} V(\alpha)-\mathbb{F} V \circ \mathbb{B} H(\alpha)),
$$

con $z: T^{*} Q \rightarrow T^{*} Q$ un morfismo de fibrados. Consideremos la descomposición ortogonal $T^{*} Q=$ $W \oplus \hat{W}$ (recordar la Observación 2.11), dada por el tensor $\mathfrak{b}_{V}$, y escribamos $z(\alpha)=z_{\|}(\alpha)-z_{\perp}(\alpha)$, $\operatorname{con} z_{\text {ll }}(\alpha) \in W \mathrm{y} z_{\perp}(\alpha) \in \hat{W}$.

Proposición 2.5. Bajo las suposiciones de esta sección, la aplicación y dada por (2.49) satisface (2.43), para alguna función no negativa $\mu$, si y sólo si $V$ satisface (2.46) y z cumple

$$
\mathfrak{b}_{V}(z(\alpha), \alpha)=-\mu(\alpha), \quad \mathfrak{b}_{V}(z(\alpha), \sigma)=\Upsilon(\alpha, \alpha, \sigma) \quad y \quad z(\bar{\alpha})=0
$$

[donde $\Upsilon$ es el tensor definido en (2.33)] para todo $\alpha \in T^{*} Q y \sigma \in \hat{W}$; o equivalentemente, $z_{\perp}$ está dado por

$$
\mathfrak{b}_{V}\left(z_{\perp}(\alpha), \sigma\right)= \begin{cases}0, & \sigma \in W \\ -\Upsilon(\alpha, \alpha, \sigma), & \sigma \in \hat{W}\end{cases}
$$

$y z_{11}$ cumple

$$
\mathfrak{b}_{V}\left(z_{\|}(\alpha), \alpha\right)=\mathfrak{b}_{V}\left(z_{\perp}(\alpha), \alpha\right)-\mu(\alpha), \quad z_{\|}(\alpha) \in W \quad y \quad z_{\|}(\bar{\alpha})=0
$$


Demostración. En primer lugar, recordemos que, partiendo de los resultados de la sección anterior (ver Teorema 2.2), sabemos que existe una solución y de (2.43) para $V$ (y alguna función no negativa $\mu$ ) si y sólo si $V$ satisface (2.46), o equivalentemente (2.47) y (2.48). Supongamos que dicha solución está dada por (2.49) y veamos qué implica esto para el morfismo $z$. Usando (1.21), tenemos que

$$
\begin{aligned}
\left\langle\mathbb{F} V^{-1} \circ(\mathbb{F} H \circ \mathbb{B} V(\alpha)-\mathbb{F} V \circ \mathbb{B} H(\alpha)), \mathbb{F} V(\alpha)\right\rangle & =\langle\alpha, \mathbb{F} H \circ \mathbb{B} V(\alpha)-\mathbb{F} V \circ \mathbb{B} H(\alpha)\rangle \\
& =\langle\mathbb{B} V(\alpha), \mathbb{F} H(\alpha)\rangle-\langle\mathbb{B} H(\alpha), \mathbb{F} V(\alpha)\rangle \\
& =\{V, H\}(\alpha) .
\end{aligned}
$$

Luego $\langle y(\alpha), \mathbb{F} V(\alpha)\rangle=\langle z(\alpha), \mathbb{F} V(\alpha)\rangle-\{V, H\}(\alpha)$ y, en consecuencia, la primera parte de (2.43) implica que

$$
\langle z(\alpha), \mathbb{F} V(\alpha)\rangle=-\mu(\alpha) .
$$

Estudiemos ahora la segunda parte de (2.43), i.e. la condición $y \subset W$. Notemos primero que, para todo $\alpha, \sigma \in T^{*} Q$ en la misma fibra,

$$
\langle y(\alpha), \mathbb{F} V(\sigma)\rangle=\langle z(\alpha), \mathbb{F} V(\sigma)\rangle-\langle\mathbb{B} V(\alpha), \mathbb{F} H(\sigma)\rangle+\langle\mathbb{B} H(\alpha), \mathbb{F} V(\sigma)\rangle .
$$

Para encontrar otra expresión equivalente de esta última ecuación, escribamos a $H$ y $V$ usando los términos cinético y potencial de cada una, y consideremos el tensor $\Upsilon: T^{*} Q \times T^{*} Q \times T^{*} Q \rightarrow \mathbb{R}$ definido en (2.33) y el tensor $\psi: T^{*} Q \rightarrow \mathbb{R}$ dado por

$$
\psi(\alpha):=\langle\mathrm{d} v(\pi(\alpha)), \mathbb{F} \mathfrak{h}(\cdot)\rangle-\langle\mathrm{d} h(\pi(\alpha)), \mathbb{F} \mathfrak{v}(\cdot)\rangle .
$$

De acuerdo con la segunda parte de (1.25), la Ec. (2.33) dice que

$$
\Upsilon(\alpha, \alpha, \sigma)=\langle\mathbb{B} \mathfrak{v}(\alpha), \mathbb{F} \mathfrak{h}(\sigma)\rangle-\langle\mathbb{B} \mathfrak{h}(\alpha), \mathbb{F} \mathfrak{v}(\sigma)\rangle,
$$

para todo $\alpha, \sigma \in T^{*} Q$ sobre la misma fibra. Luego, las Ecs. (2.47) y (2.48) toman la forma

$$
\Upsilon(\sigma, \sigma, \sigma)=\psi(\sigma)=0, \quad \forall \sigma \in \hat{W} .
$$

Por otro lado, dado que

$$
\begin{aligned}
& \langle\mathbb{B} V(\alpha), \mathbb{F} H(\sigma)\rangle-\langle\mathbb{B} H(\alpha), \mathbb{F} V(\sigma)\rangle=\langle\mathbb{B} \mathfrak{v}(\alpha), \mathbb{F} \mathfrak{h}(\sigma)\rangle-\langle\mathbb{B} \mathfrak{h}(\alpha), \mathbb{F} \mathfrak{v}(\sigma)\rangle+ \\
& +(\langle\mathrm{d} v(q), \mathbb{F} \mathfrak{h}(\cdot)\rangle-\langle\mathrm{d} h(q), \mathbb{F} \mathfrak{v}(\cdot)\rangle)=\Upsilon(\alpha, \alpha, \sigma)+\psi(\sigma),
\end{aligned}
$$

usando (2.54), tenemos que

$$
\langle y(\alpha), \mathbb{F} V(\sigma)\rangle=\langle z(\alpha), \mathbb{F} V(\sigma)\rangle-\Upsilon(\alpha, \alpha, \sigma)-\psi(\sigma) .
$$

Volviendo a (2.43), la condición $y \subset W$ implica que, para todo $\sigma \in \hat{W}=\mathbb{F} V^{-1}\left(W^{\circ}\right)$ [ver la Ec. (2.55)],

$$
0=\langle y(\alpha), \mathbb{F} V(\sigma)\rangle=\langle z(\alpha), \mathbb{F} V(\sigma)\rangle-\Upsilon(\alpha, \alpha, \sigma)-\psi(\sigma) \text {. }
$$


Pero, dado que $\psi(\sigma)=0$ para todo $\sigma \in \hat{W}$,

$$
\langle z(\alpha), \mathbb{F} V(\sigma)\rangle=\Upsilon(\alpha, \alpha, \sigma), \quad \forall \alpha \in T^{*} Q, \sigma \in \hat{W} .
$$

Por lo tanto, el morfismo de fibrados $z$ debe cumplir las ecuaciones [ver (2.53) y (2.56)]

$$
\langle z(\alpha), \mathbb{F} V(\alpha)\rangle=-\mu(\alpha) \quad \text { y } \quad\langle z(\alpha), \mathbb{F} V(\sigma)\rangle=\Upsilon(\alpha, \alpha, \sigma),
$$

o equivalentemente, en términos del tensor no degenerado $\mathfrak{b}_{V}$, las primeras dos ecuaciones en (2.50). Dado que $V$ es definida positiva alrededor de $\bar{\alpha}$, y consecuentemente $\mathrm{d} V(\bar{\alpha})=0$, es sencillo probar partiendo de (2.49) que la condición $y(\bar{\alpha})=0$ implica $z(\bar{\alpha})=0$, que es la tercera ecuación de (2.50).

Escribiendo ahora $z(\alpha)=z_{\|}(\alpha)-z_{\perp}(\alpha)$ como se explicó más arriba, la Ec. (2.50) se traduce como [ver la Ec. (2.25)]

$$
\mathfrak{b}_{V}\left(z_{\|}(\alpha), \alpha\right)=\mathfrak{b}_{V}\left(z_{\perp}(\alpha), \alpha\right)-\mu(\alpha) \quad \text { y } \quad \mathfrak{b}_{V}\left(z_{\perp}(\alpha), \sigma\right)=-\Upsilon(\alpha, \alpha, \sigma),
$$

para todo $\alpha \in T^{*} Q$ y $\sigma \in \hat{W}$. La segunda parte de (2.57) dice precisamente que $z_{\perp}$ satisface (2.51). En particular, vale que $z_{\perp}\left(0_{q}\right)=0$ para todo $q \in Q$ y por lo tanto $z_{\perp}(\bar{\alpha})=0$, de manera que también se cumple $z_{\|}(\bar{\alpha})=0$. Luego, concluimos que $z_{\| \prime}$ debe cumplir todas las condiciones en (2.52).

Recíprocamente, es fácil ver que, si $z$ (resp. $z_{\perp} \mathrm{y} z_{\| 1}$ ) satisface la Ecuación (2.50) [resp. las Ecuaciones (2.51) y (2.52)] y $V$ es solución de (2.46), revirtiendo los pasos del argumento anterior se ve que $y$ cumple (2.43).

Teniendo todo esto en cuenta, podemos definir un nuevo método de estabilización (incluido en el LCB).

Definición 2.5. Dado un sistema subactuado $(H, \mathcal{W})$, con $H$ simple y $\mathcal{W}$ dado por un subfibrado $W \subset$ $T^{*} Q$, y dado un punto crítico $\bar{\alpha} \in T^{*} Q$ de $X_{H}$, el método LCB simple consiste en:

- hallar una función simple $V$ que resuelva (2.46), con $V$ definida positiva alrededor de $\bar{\alpha}$;

- fijar un morfismo de fibrados $z_{\|}: T^{*} Q \rightarrow T^{*} Q$ siguiendo los pasos de i a iii de la Sección $\$ 2.2 .4$ [ver la Ec. (2.30)];

- definir $z:=z_{\|}-z_{\perp}$, con $z_{\perp}$ dado por la Ec. (2.51); y finalmente

- definir $y$ como en la Ec. (2.49) e $Y \in \mathfrak{X}\left(T^{*} Q\right)$ como el levantamiento vertical de $y$.

\subsubsection{Maximalidad y equivalencia}

Los cálculos de la sección anterior, junto con el Teorema 2.1 tienen como consecuencia una propiedad de maximalidad para el método LCB simple (ver la Definición 2.5). En efecto, el LCB simple resulta maximal cuando se considera dentro de la clase de métodos de estabilización de Lyapunov en los cuales la función de Lyapunov asociada puede elegirse como una función simple. Enunciamos esta propiedad de manera precisa en el siguiente teorema. 
Teorema 2.3. Consideremos un sistema subactuado $(H, \mathcal{W})$, con $H$ simple, $\mathcal{W}$ dada por un subfibrado vectorial $W \subset T^{*} Q$, y un punto crítico $\bar{\alpha} \in T^{*} Q$ de $X_{H}$. Si $Y \subset \mathcal{W}$ es un campo vectorialy $V$ es una función de Lyapunov simple para $X_{H}+Y y \bar{\alpha}$, entonces $V$ cumple la Ec. (2.46), es definida positiva alrededor de $\bar{\alpha}, y$ además $Y$ está dado por el método LCB simple.

Esta propiedad implica que el método $\mathrm{CH}$ simple (ver Definición 2.4) está contenido en el método LCB simple (recordar la Definición 2.2). En lo que sigue mostraremos que la inclusión inversa también se cumple, de modo que ambos métodos resultan equivalentes.

Consideremos el conjunto $\mathfrak{U}$ de ternas $(H, \mathcal{W}, \bar{\alpha})$, donde $H$ es una función simple, $\mathcal{W}$ está dado por un subfibrado vectorial $W \subset T^{*} Q$, y $\bar{\alpha} \in T^{*} Q$ es un punto crítico de $X_{H}$. Notemos que los métodos LCB y $\mathrm{CH}$, en sus versiones simples, son métodos de estabilización definidos sobre $\mathfrak{U}$ (ver la Definición 2.1). Denotemos por $\digamma$ y $\digamma^{\prime}$ las asignaciones asociadas al $\mathrm{LCB}$ y al $\mathrm{CH}$, respectivamente; que determinan el conjunto de señales de control que resuelven el problema $\mathbf{P}$. Veamos que

$$
\digamma(H, \mathcal{W}, \bar{\alpha}) \subset \digamma^{\prime}(H, \mathcal{W}, \bar{\alpha}), \quad \forall(H, \mathcal{W}, \bar{\alpha}) \in \mathfrak{U} .
$$

Sea $Y \in \mathfrak{X}\left(T^{*} Q\right)$ un campo vectorial relacionado con $(H, \mathcal{W}, \bar{\alpha})$ por el método LCB simple, i.e. $Y \in \digamma(H, \mathcal{W}, \bar{\alpha})$. Luego, $Y$ es el levantamiento vertical de un morfismo de fibrados $y$ dado por (2.49), con $V$ simple y definida positiva alrededor de $\bar{\alpha}$, cumpliendo (2.46), y $z=z_{\| \prime}-z_{\perp}$ con $z_{\perp}$ dado por la Ec. (2.51) y $z_{\|}$de la forma (2.30). Queremos ver que $Y \in \digamma^{\prime}(H, \mathcal{W}, \bar{\alpha})$. Para ello, es suficiente mostrar que, comparando (2.29) y (2.49),

$$
z(\alpha)=z_{d}(\alpha)+\mathfrak{Z}_{g}\left(\alpha, \alpha, \mathbb{F} V^{-1}(\cdot)\right)
$$

para algún morfismo de fibrados $z_{d}$ de la forma (2.30) y algún tensor $\mathfrak{Z}_{g}$ dado por (2.34). Para ello, podemos definir

$$
\left\{\begin{array}{l}
\mathfrak{Z}_{g}\left(\alpha_{1}, \alpha_{2}, \sigma\right):=\Upsilon\left(\alpha_{1}, \alpha_{2}, \sigma\right), \mathfrak{Z}_{g}\left(\sigma_{1}, \sigma_{2}, \gamma\right):=-\Upsilon\left(\gamma, \sigma_{2}, \sigma_{1}\right)-\Upsilon\left(\gamma, \sigma_{1}, \sigma_{2}\right), \\
\mathfrak{Z}_{g}\left(\gamma_{1}, \sigma, \gamma_{2}\right):=\mathfrak{Z}_{g}\left(\sigma, \gamma_{1}, \gamma_{2}\right):=-\frac{1}{2} \Upsilon\left(\gamma_{1}, \gamma_{2}, \sigma\right), \quad \mathfrak{Z}_{g}\left(\gamma_{1}, \gamma_{2}, \gamma\right):=0,
\end{array}\right.
$$

$\operatorname{con} \alpha_{i} \in T^{*} Q, \sigma, \sigma_{i} \in \hat{W} \mathrm{y} \gamma, \gamma_{i} \in W$ si $i=1,2, \mathrm{y}$

$$
z_{d}(\alpha):=z_{\|}(\alpha)-\mathfrak{Z}_{g}\left(\alpha, \alpha, \mathbb{F} V^{-1}(\cdot)\right) .
$$

De hecho, usando (2.50) y la definición de $\mathfrak{Z}_{g}$,

$$
\mathfrak{b}_{V}\left(z_{d}(\alpha), \alpha\right):=\mathfrak{b}_{V}(z(\alpha), \alpha)-\mathfrak{Z}_{g}(\alpha, \alpha, \alpha)=-\mu(\alpha)+0=-\mu(\alpha),
$$

para todo $\alpha \in T^{*} Q$

$$
\mathfrak{b}_{V}\left(z_{d}(\alpha), \sigma\right):=\mathfrak{b}_{V}(z(\alpha), \sigma)-\mathfrak{Z}_{g}(\alpha, \alpha, \sigma)=\Upsilon(\alpha, \alpha, \sigma)-\Upsilon(\alpha, \alpha, \sigma)=0,
$$

para todo $\sigma \in \hat{W}$, y

$$
z_{d}(\bar{\alpha}):=z(\bar{\alpha})-\mathfrak{Z}_{g}\left(\bar{\alpha}, \bar{\alpha}, \mathbb{F} V^{-1}(\cdot)\right)=0-0=0,
$$

lo cual implica que $z_{d}$ cumple (2.30). Esto prueba el resultado que exponemos a continuación. 
Teorema 2.4. Los métodos $L C B$ y CH, en sus versiones simples, son métodos de estabilización equivalentes.

Como conclusión, mencionemos una consecuencia directa (y a la vez importante) sobre el método $\mathrm{CH}$ simple, que se deduce de los Teoremas 2.3 y 2.4: dada una terna $(H, \mathcal{W}, \bar{\alpha}) \in \mathfrak{U}$, toda ley de control $Y \subset \mathcal{W}$ para la cual existe una función de Lyapunov simple para $X_{H}+Y$ y $\bar{\alpha}$, puede construirse mediante el $\mathrm{CH}$ simple. En pocas palabras, dentro del mundo de los sistemas Hamiltonianos simples con actuadores, si queremos estabilizar uno de tales sistemas exhibiendo una función de Lyapunov simple, el método CH simple (y, por supuesto, el método LCB simple) es la manera más general de hacerlo. Según entendemos, esta es la primera vez que tal afirmación es enunciada. 


\section{ESTABILIZABILIDAD ASINTÓTICA DE}

\section{SISTEMAS CON DOS GRADOS DE LIBERTAD}

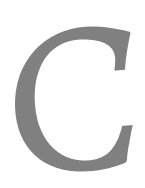

ONSIDEREMOS un sistema Hamiltoniano 1-subactuado sobre una variedad $Q$ de dimensión $n$, es decir, supongamos que las fibras del espacio de actuación $\mathcal{W}$ tienen dimensión $n-1$. Supongamos que su Hamiltoniano asociado $H$ es simple (i.e. es de la forma energía cinética más energía potencial) y fijemos un punto crítico $\bar{\alpha}$ del campo vectorial Hamiltoniano $X_{H}$ asociado a $H$. En la Referencia [12], D.E. Chang encontró condiciones suficientes que aseguran la estabilizabilidad de un tal sistema en $\bar{\alpha}$, por medio de la existencia de una función de Lyapunov (simple) para $X_{H}$ y $\bar{\alpha}$. Concretamente, fijando coordenadas locales $(U, \varphi)$ en las cuales el punto crítico cumpla $\left(\varphi^{*}\right)^{-1}(\bar{\alpha})=$ $(0, \ldots, 0)$, el espacio de actuación esté generado por el conjunto $\left\{\mathrm{d} q^{1+a}\right\}_{a=1}^{n-1}$ y $H$ se escriba

$$
H(\mathbf{q}, \mathbf{p})=\frac{1}{2} p_{i} \mathbb{H}^{i j}(\mathbf{q}) p_{j}+h(\mathbf{q}) ;
$$

tal condición puede expresarse como:

$$
\left\{\begin{array}{l}
\left(\sum_{i=1}^{n} \mathbb{H}^{1+a, i} \frac{\partial^{2} h}{\partial q^{1} \partial q^{i}}\right)(0, \ldots, 0) \neq 0, \quad \text { para algún } \quad 1 \leq a \leq n-1, \quad \text { o bien } \\
\frac{\partial^{2} h}{\left(\partial q^{1}\right)^{2}}(0, \ldots, 0)>0
\end{array}\right.
$$

En términos intrínsecos, la primera de las expresiones en (3.1) dice que la linealización del sistema Hamiltoniano subactuado alrededor de $(0, \ldots, 0)$ es controlable, mientras que la segunda expresa que dicha linealización no es controlable y sus modos no controlables están dados por un par imaginario puro.

Más aun, en el caso de sistemas con dos grados de libertad, la Referencia [13] presenta dos condiciones adicionales que permiten asegurar no solo la estabilizabilidad, sino también la estabilizabilidad asintótica. Todos estos resultados fueron desarrollados en el contexto del método del $\mathrm{CH}^{1}$ simple que presentamos en el capítulo 2 (ver Def. 2.4).

\footnotetext{
${ }^{1}$ Más precisamente, su contraparte Lagrangiana: el método de los Lagrangianos controlados.
} 
En este capítulo vamos a presentar una derivación alternativa de la condición de estabilizabilidad que mencionamos arriba [Ec. (3.1)], pero en el contexto del método LCB simple (ver Def. 2.5). Teniendo en cuenta las características del método LCB simple, esto es equivalente a estudiar la existencia de soluciones definidas positivas de las ecuaciones cinéticas y potenciales. De hecho, el resultado hallado por Chang se basa en el estudio de la existencia de soluciones definidas positivas de las condiciones de matching simples.

Para realizar este estudio introduciremos un cambio de variable dependiente en las incógnitas del término cinético $\mathbb{V}$ que resultará en una simplifación importante de las ecuaciones cinéticas y potenciales. Al trabajar con el LCB en lugar del CH, podremos mostrar que la condición (3.1) no es solamente suficiente para la estabilizabilidad, sino también necesaria si se pretende exhibir una función de Lyapunov simple que garantice dicha estabilidad. Más precisamente, si queremos estabilizar un sistema Hamiltoniano 1-subactuado construyendo una función de Lyapunov simple para el sistema a lazo cerrado resultante, entonces la condición (3.1) debe cumplirse para el sistema no actuado.

Vale la pena destacar que los resultados de existencia de soluciones definidas positivas fueron obtenidos independientemente de los análogos para el método $\mathrm{CH}$ simple. De hecho, fueron probados antes de saber que ambos conjuntos de PDEs son de hecho el mismo, ${ }^{2}$ como se probó en el Capítulo 2.

Adicionalmente probaremos que, en el caso de sistemas con dos grados de libertad, la condición (3.1) no solamente asegura estabilizabilidad, sino también estabilizabilidad asintótica. En otras palabras, no es necesaria una condición adicional, además de (3.1), para estabilizar asintóticamente un sistema Hamiltoniano subactuado con dos grados de libertad. Este es el resultado principal de este capítulo y fue probado, junto con la derivación alternativa de las condiciones de estabilidad para sistemas 1subactuados, en la Referencia [24].

El capítulo está organizado de la siguiente manera. En primer lugar, introduciremos los sistemas regularmente actuados y reescribiremos las ecuaciones cinéticas y potenciales para esta subclase de sistemas que, en particular, incluye a los 1-subactuados. En la Sección \$3.1, estudiaremos la existencia de soluciones para tales ecuaciones en el caso de subactuación 1 y probaremos que (3.1) es condición necesaria y suficiente para la estabilizabilidad de un tal sistema. En la última sección, combinaremos el método LCB, el principio de invariancia de LaSalle, un algoritmo à la Dirac y el Lema de Morse y mostraremos que (3.1) también es suficiente para concluir la estabilizabilidad asintótica en el caso de sistemas con dos grados de libertad. El capítulo finaliza con un ejemplo que ilustra estos resultados.

Aclaración: para simplificar notación escribiremos $0:=(0, \ldots, 0) \in \mathbb{R}^{n}$ y nos referiremos a este elemento como "origen". De manera análoga utilizaremos la notación $(\mathbf{0}, \mathbf{0}):=(0, \ldots, 0) \in \mathbb{R}^{2 n}$.

Por otra parte, todos los espacios de actuación $\mathcal{W}$ considerados en este capítulo estarán definidos a partir de un subfibrado vectorial de $T^{*} Q$, i.e. $\mathcal{W}_{\alpha}=\operatorname{vlift}_{\alpha}\left(W_{\pi(\alpha)}\right)$. Los Hamiltonianos $H$ y las funciones de Lyapunov $V$ consideradas de ahora en adelante serán siempre simples (salvo que se aclare ex-

\footnotetext{
${ }^{2}$ Esto no debería resultar sorprendente, puesto que la forma de las condiciones de matching obtenidas originalmente por Chang es sensiblemente distinta a la forma de las ecuaciones cinéticas y potenciales del LCB.
} 
presamente lo contrario). Además, todas las cartas coordenadas utilizadas en expresiones locales serán cartas centradas en el punto crítico $\bar{\alpha}$. Más precisamente, si $\bar{q}=\pi(\bar{\alpha})$ y $(U, \varphi)$ es una carta coordenada alrededor de $\bar{q}$, consideraremos $\varphi(\bar{q})=\mathbf{0}$. Como hemos hecho en los capítulos anteriores, utilizaremos las cartas inducidas en el cotangente, de modo que $\left(\varphi^{*}\right)^{-1}(\bar{\alpha})=(\mathbf{0}, \mathbf{0})$.

\subsection{Ecuaciones cinéticas y potenciales para sistemas regularmente actua- dos}

Fijemos un sistema subactuado $(H, \mathcal{W})$ con $H$ simple y $\mathcal{W}$ dado por un subfibrado $W \subseteq T^{*} Q$. Supondremos además que $W$ es integrable, es decir, si $W$ está generado localmente por formas diferenciales $\xi^{1}, \ldots, \xi^{m}$, supondremos que el ideal algebraico $\mathcal{I}$ generado por $\xi^{1}, \ldots, \xi^{m}$ es también un ideal diferencial, i.e. $\mathrm{d} \mathcal{I} \subset \mathcal{I}$. Para tales subfibrados $W$, puede verse que existe una carta coordenada local $(U, \varphi)$ de $Q$ tal que

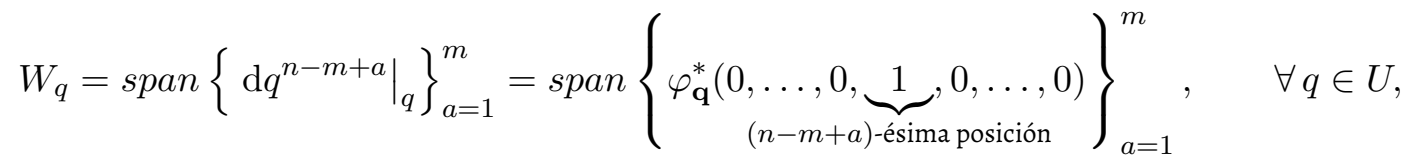

donde $n:=\operatorname{dim} Q$ y $m$ es el rango de $W$.

Definición 3.1. Un sistema Hamiltoniano subactuado $(H, \mathcal{W})$ se dice regularmente actuado si la distribución asociada $W$ es integrable. En tal caso, diremos que una carta coordenada $(U, \varphi)$ cumpliendo (3.2) es una carta adaptada a $W$.

En lo que sigue, valiéndonos de las coordenadas que acabamos de introducir, encontraremos una nueva expresión local para las ecuaciones cinéticas y potenciales para el caso de sistemas regularmente actuados. Para ello, denotaremos $s=n-m$ y usaremos la siguiente convención de índices:

$$
\left\{\begin{aligned}
i, j, k, l & =1, \ldots, n, \\
\mu, \nu, \sigma, \tau & =1, \ldots, s, \\
a, b, c, d, e & =1, \ldots, m, \\
x, y, z & =1, \ldots, m-1 .
\end{aligned}\right.
$$

Como hemos hecho anteriormente, utilizaremos la siguiente notación para las expresiones locales de $H$ y $V$ :

$$
H(\mathbf{q}, \mathbf{p})=\frac{1}{2} p_{i} \mathbb{H}^{i j}(\mathbf{q}) p_{j}+h(\mathbf{q})
$$

y

$$
V(\mathbf{q}, \mathbf{p})=\frac{1}{2} p_{i} \mathbb{V}^{i j}(\mathbf{q}) p_{j}+v(\mathbf{q})
$$

y omitiremos la dependencia de $\mathbf{q}$ de las funciones $\mathbb{H}, h, \mathbb{V}$ y $v$ para simplificar la notación. 
Recordemos que las ecuaciones cinéticas y potenciales se escriben

$$
\left(\frac{\partial \mathbb{V}^{i j}}{\partial q^{k}} \mathbb{H}^{k l}-\frac{\partial \mathbb{H}^{i j}}{\partial q^{k}} \mathbb{V}^{k l}\right) p_{i} p_{j} p_{l}=0
$$

y

$$
\left(\frac{\partial v}{\partial q^{k}} \mathbb{H}^{k l}-\frac{\partial h}{\partial q^{k}} \mathbb{V}^{k l}\right) p_{l}=0
$$

respectivamente. Ambas ecuaciones deben ser resueltas para todo $\mathbf{p} \in\left(\varphi^{*}\right)^{-1}(\hat{W})$ y para todo $\mathbf{q} \in$ $\varphi(U)$. Dado que $\left(\varphi^{*}\right)^{-1}(\hat{W})$ es el complemento ortogonal de $\left(\varphi^{*}\right)^{-1}(W)$ respecto de $\mathbb{V}$, la condición $\mathbf{p} \in\left(\varphi^{*}\right)^{-1}(\hat{W})$ es equivalente a

$$
\mathbb{V}^{s+a, i} p_{i}=0, \quad \text { para cada } a=1, \ldots, m
$$

Dado que $\mathbb{V}$ es una matriz simétrica y definida positiva de dimensión $n$, si $r \leq n$ es un entero positivo, sabemos que toda submatriz $\mathbb{V}_{\underline{\underline{r}}}$ dada por

$$
\mathbb{V}_{\underline{r}}^{x y}:=\mathbb{V}^{n-r+x, n-r+y} \quad \forall 1 \leq x, y \leq r .
$$

es, a su vez, simétrica y definida positiva (ver Apéndice). En particular, cada una de estas matrices es invertible y su inversa es también simétrica y definida positiva. Tomando $r=m$ es claro que $\left(\mathbb{V}_{\underline{m}}\right)_{a c} \mathbb{V}_{\underline{m}}^{c b}=$ $\delta_{a}^{b}$

Observación 3.1. Es importante notar la diferencia entre los símbolos $\mathbb{V}_{a b} \mathrm{y}\left(\mathbb{V}_{\underline{m}}\right)_{a b}$. El primero denota el elemento " $a, b$ " de la inversa de $\mathbb{V}$, mientras que el segundo denota el elemento " $a, b$ " de la inversa de la matriz $\mathbb{V}_{\underline{m}}$. En consecuencia, cabe destacar que los números $\left(\mathbb{V}_{\underline{m}}\right)_{a b}$ dependen exclusivamente de los números $\mathbb{V}_{\underline{m}}^{a b}=\mathbb{V}^{s+a, s+b}$ y no de los números $\mathbb{V}^{\mu \nu}$.

En estas condiciones, y usando la Ecuación (3.5),

$$
\begin{aligned}
\mathbb{V}^{s+a, i} p_{i} & =0 \Rightarrow \\
\mathbb{V}^{s+a, \mu} p_{\mu}+\mathbb{V}^{s+a, s+b} p_{s+b} & =0 \Rightarrow \\
\mathbb{V}^{s+a, s+b} p_{s+b} & =-\mathbb{V}^{s+a, \mu} p_{\mu} \Rightarrow \\
p_{s+b} & =-\left(\mathbb{V}_{\underline{m}}\right)_{b a} \mathbb{V}^{s+a, \mu} p_{\mu} .
\end{aligned}
$$

Luego, definiendo

$$
\gamma_{b}^{\mu}:=\left(\mathbb{V}_{\underline{m}}\right)_{b a} \mathbb{V}^{s+a, \mu}
$$

la condición (3.5) es equivalente a

$$
p_{s+b}=-\gamma_{b}^{\mu} p_{\mu} .
$$

Estudiemos ahora las ecuaciones cinéticas. Tomando el primer término entre paréntesis, escribamos

$$
\frac{\partial \mathbb{V}^{i j}}{\partial q^{k}} \mathbb{H}^{k l} p_{i} p_{j} p_{l}=\left(p_{i} \frac{\partial \mathbb{V}^{i j}}{\partial q^{k}} p_{j}\right)\left(\mathbb{H}^{k l} p_{l}\right)
$$


y analicemos cada factor por separado. En primer lugar,

$$
\mathbb{H}^{k l} p_{l}=\mathbb{H}^{k \sigma} p_{\sigma}+\mathbb{H}^{k, s+b} p_{s+b}=\mathbb{H}^{k \sigma} p_{\sigma}-\mathbb{H}^{k, s+b} \gamma_{b}^{\sigma} p_{\sigma}=\left(\mathbb{H}^{k \sigma}-\mathbb{H}^{k, s+b} \gamma_{b}^{\sigma}\right) p_{\sigma},
$$

donde hemos usado (3.6) y (3.7). Por otro lado,

$$
\begin{aligned}
p_{i} \frac{\partial \mathbb{V}^{i j}}{\partial q^{k}} p_{j} & =p_{i}\left(\frac{\partial \mathbb{V}^{i \nu}}{\partial q^{k}} p_{\nu}+\frac{\partial \mathbb{V}^{i, s+b}}{\partial q^{k}} p_{s+b}\right) \\
& =p_{i}\left(\frac{\partial \mathbb{V}^{i \nu}}{\partial q^{k}} p_{\nu}-\frac{\partial \mathbb{V}^{i, s+b}}{\partial q^{k}} \gamma_{b}^{\nu} p_{\nu}\right) \\
& =p_{i}\left(\frac{\partial \mathbb{V}^{i \nu}}{\partial q^{k}}-\frac{\partial \mathbb{V}^{i, s+b}}{\partial q^{k}} \gamma_{b}^{\nu}\right) p_{\nu} \\
& =\left[p_{\mu}\left(\frac{\partial \mathbb{V}^{\mu \nu}}{\partial q^{k}}-\frac{\partial \mathbb{V}^{\mu, s+b}}{\partial q^{k}} \gamma_{b}^{\nu}\right)+p_{s+a}\left(\frac{\partial \mathbb{V}^{s+a, \nu}}{\partial q^{k}}-\frac{\partial \mathbb{V}^{s+a, s+b}}{\partial q^{k}} \gamma_{b}^{\nu}\right)\right] p_{\nu} \\
& =\left[p_{\mu}\left(\frac{\partial \mathbb{V}^{\mu \nu}}{\partial q^{k}}-\frac{\partial \mathbb{V}^{\mu, s+b}}{\partial q^{k}} \gamma_{b}^{\nu}\right)-\gamma_{a}^{\mu} p_{\mu}\left(\frac{\partial \mathbb{V}^{s+a, \nu}}{\partial q^{k}}-\frac{\partial \mathbb{V}^{s+a, s+b}}{\partial q^{k}} \gamma_{b}^{\nu}\right)\right] p_{\nu} \\
& =p_{\mu}\left[\frac{\partial \mathbb{V}^{\mu \nu}}{\partial q^{k}}-\frac{\partial \mathbb{V}^{\mu, s+b}}{\partial q^{k}} \gamma_{b}^{\nu}-\gamma_{a}^{\mu} \frac{\partial \mathbb{V}^{s+a, \nu}}{\partial q^{k}}+\gamma_{a}^{\mu} \frac{\partial \mathbb{V}^{s+a, s+b}}{\partial q^{k}} \gamma_{b}^{\nu}\right] p_{\nu} .
\end{aligned}
$$

Efectuando un cambio de índices mudos y utilizando (3.6),

$$
\begin{aligned}
p_{i} \frac{\partial \mathbb{V}^{i j}}{\partial q^{k}} p_{j} & =p_{\mu}\left[\frac{\partial \mathbb{V}^{\mu \nu}}{\partial q^{k}}-\frac{\partial \mathbb{V}^{\mu, s+b}}{\partial q^{k}}\left(\mathbb{V}_{\underline{m}}\right)_{b d} \mathbb{V}^{s+d, \nu}-\left(\mathbb{V}_{\underline{m}}\right)_{d b} \mathbb{V}^{s+b, \mu} \frac{\partial \mathbb{V}^{s+d, \nu}}{\partial q^{k}}\right. \\
& \left.+\mathbb{V}^{\mu, s+b}\left(\mathbb{V}_{\underline{m}}\right)_{e b} \frac{\partial \mathbb{V}^{s+e, s+a}}{\partial q^{k}}\left(\mathbb{V}_{\underline{m}}\right)_{a d} \mathbb{V}^{\nu, s+d}\right] p_{\nu} \\
& =p_{\mu}\left[\frac{\partial \mathbb{V}^{\mu \nu}}{\partial q^{k}}-\left(\mathbb{V}_{\underline{m}}\right)_{b d} \frac{\partial\left(\mathbb{V}^{\left.\mu, s+b \mathbb{V}^{\nu, s+d}\right)}\right.}{\partial q^{k}}-\mathbb{V}^{\mu, s+b} \frac{\partial\left(\mathbb{V}_{\underline{m}}\right)_{b d}}{\partial q^{k}} \mathbb{V}^{\nu, s+d}\right] p_{\nu} \\
& =p_{\mu}\left[\frac{\partial \mathbb{V}^{\mu \nu}}{\partial q^{k}}-\frac{\partial\left(\mathbb{V}^{\mu, s+b}\left(\mathbb{V}_{\underline{m}}\right)_{b d} \mathbb{V}^{\nu, s+d}\right)}{\partial q^{k}}\right] p_{\nu} \\
& =p_{\mu} \frac{\partial\left(\mathbb{V}^{\mu \nu}-\mathbb{V}^{\mu, s+b}\left(\mathbb{V}_{\underline{m}}\right)_{b d} \mathbb{V}^{\nu, s+d}\right)}{\partial q^{k}} p_{\nu},
\end{aligned}
$$

donde hemos usado la simetría de $\mathbb{V}$ y $\mathbb{V}_{\underline{m}}$. Luego, si definimos

$$
\delta^{\mu \nu}:=\mathbb{V}^{\mu \nu}-\mathbb{V}^{\mu, s+b}\left(\mathbb{V}_{\underline{m}}\right)_{b d} \mathbb{V}^{\nu, s+d},
$$

y

$$
A^{\sigma k}:=\mathbb{H}^{k \sigma}-\mathbb{H}^{k, s+b} \gamma_{b}^{\sigma},
$$

tenemos que

$$
\frac{\partial \mathbb{V}^{i j}}{\partial q^{k}} \mathbb{H}^{k l} p_{i} p_{j} p_{l}=A^{\sigma k} \frac{\partial \delta^{\mu \nu}}{\partial q^{k}} p_{\mu} p_{\nu} p_{\sigma} .
$$

Analicemos ahora el segundo término de las ecuaciones cinéticas. De manera análoga, escribamos

$$
\frac{\partial \mathbb{H}^{i j}}{\partial q^{k}} \mathbb{V}^{k l} p_{i} p_{j} p_{l}=\left(p_{i} \frac{\partial \mathbb{H}^{i j}}{\partial q^{k}} p_{j}\right)\left(\mathbb{V}^{k l} p_{l}\right)
$$


Nuevamente analizamos por separado y hallamos

$$
\mathbb{V}^{k l} p_{l}=\mathbb{V}^{k l} p_{l}=\mathbb{V}^{k \sigma} p_{\sigma}+\mathbb{V}^{k, s+a} p_{s+a}=\left(\mathbb{V}^{k \sigma}-\mathbb{V}^{k, s+a}\left(\mathbb{V}_{\underline{m}}\right)_{a b} \mathbb{V}^{s+b, \sigma}\right) p_{\sigma},
$$

para el segundo término entre paréntesis y

$$
\left(p_{i} \frac{\partial \mathbb{H}^{i j}}{\partial q^{k}} p_{j}\right)=\left(\frac{\partial \mathbb{H}^{\mu \nu}}{\partial q^{k}}-\frac{\partial \mathbb{H}^{\mu, s+b}}{\partial q^{k}} \gamma_{b}^{\nu}-\frac{\partial \mathbb{H}^{\nu, s+a}}{\partial q^{k}} \gamma_{a}^{\mu}+\gamma_{a}^{\mu} \gamma_{b}^{\nu} \frac{\partial \mathbb{H}^{s+a, s+b}}{\partial q^{k}}\right) p_{\mu} p_{\nu},
$$

para el primero. Juntando ambas expresiones, se sigue que

$$
\begin{aligned}
\left(p_{i} \frac{\partial \mathbb{H}^{i j}}{\partial q^{k}} p_{j}\right)\left(\mathbb{V}^{k l} p_{l}\right) & =\left(\frac{\partial \mathbb{H}^{\mu \nu}}{\partial q^{k}}-\frac{\partial \mathbb{H}^{\mu, s+b}}{\partial q^{k}} \gamma_{b}^{\nu}-\frac{\partial \mathbb{H}^{\nu, s+a}}{\partial q^{k}} \gamma_{a}^{\mu}+\gamma_{a}^{\mu} \gamma_{b}^{\nu} \frac{\partial \mathbb{H}^{s+a, s+b}}{\partial q^{k}}\right)\left(\mathbb{V}^{k \sigma}\right. \\
& \left.-\mathbb{V}^{k, s+a}\left(\mathbb{V}_{\underline{m}}\right)_{a b} \mathbb{V}^{s+b, \sigma}\right) p_{\mu} p_{\nu} p_{\sigma} \\
& =\left(\frac{\partial \mathbb{H}^{\mu \nu}}{\partial q^{\tau}}-\frac{\partial \mathbb{H}^{\mu, s+b}}{\partial q^{\tau}} \gamma_{b}^{\nu}-\frac{\partial \mathbb{H}^{\nu, s+a}}{\partial q^{\tau}} \gamma_{a}^{\mu}+\gamma_{a}^{\mu} \gamma_{b}^{\nu} \frac{\partial \mathbb{H}^{s+a, s+b}}{\partial q^{\tau}}\right)\left(\mathbb{V}^{\tau \sigma}\right. \\
& \left.-\mathbb{V}^{\tau, s+a}\left(\mathbb{V}_{\underline{m}}\right)_{a b} \mathbb{V}^{s+b, \sigma}\right) p_{\mu} p_{\nu} p_{\sigma} \\
& =\left(\frac{\partial \mathbb{H}^{\mu \nu}}{\partial q^{\tau}}-\frac{\partial \mathbb{H}^{\mu, s+b}}{\partial q^{\tau}} \gamma_{b}^{\nu}-\frac{\partial \mathbb{H}^{\nu, s+a}}{\partial q^{\tau}} \gamma_{a}^{\mu}+\gamma_{a}^{\mu} \gamma_{b}^{\nu} \frac{\partial \mathbb{H}^{s+a, s+b}}{\partial q^{\tau}}\right) \delta^{\tau \sigma} p_{\mu} p_{\nu} p_{\sigma}
\end{aligned}
$$

Por lo tanto, definiendo

$$
B_{\tau}^{\mu \nu}:=\frac{\partial \mathbb{H}^{\mu \nu}}{\partial q^{\tau}}-\frac{\partial \mathbb{H}^{\mu, s+b}}{\partial q^{\tau}} \gamma_{b}^{\nu}-\frac{\partial \mathbb{H}^{\nu, s+a}}{\partial q^{\tau}} \gamma_{a}^{\mu}+\gamma_{a}^{\mu} \gamma_{b}^{\nu} \frac{\partial \mathbb{H}^{s+a, s+b}}{\partial q^{\tau}}
$$

las ecuaciones cinéticas se escriben

$$
\left(A^{\sigma k} \frac{\partial \delta^{\mu \nu}}{\partial q^{k}}-B_{\tau}^{\mu \nu} \delta^{\tau \sigma}\right) p_{\mu} p_{\nu} p_{\sigma}=0, \quad \forall\left(p_{1}, \ldots, p_{s}\right) \in \mathbb{R}^{s} .
$$

Observación 3.2. Notemos que tanto $\delta^{\mu \nu} \operatorname{como} B_{\tau}^{\mu \nu}$ son simétricos en los índices $\mu \mathrm{y} \nu$ [ver (3.8) y (3.10)].

Observación 3.3. Los números $A^{\sigma k}$ y $B_{\tau}^{\mu \nu}$ pueden entenderse como las componentes de dos tensores que denotaremos $A$ y $B$, respectivamente.

Observemos que la Ecuación (3.11) se trata de la anulación de un polinomio homogéneo de grado 3 en las variables $p_{1}, \ldots, p_{s}$. Es un hecho conocido que la anulación de un tal polinomio es equivalente a la anulación de cada uno de los coeficientes correspondientes a cada monomio. Tales coeficientes se obtienen como la simetrización en los índices $\mu, \nu$ y $\sigma$ del término entre paréntesis en (3.11). A su vez, la simetrización se obtiene sumando dicho término sobre todas las permutaciones de los índices involucrados. Sin embargo, siguiendo la Observación 3.2, basta tomar solamente la suma sobre las permutaciones cíclicas. Llegamos así a una nueva expresión para las ecuaciones cinéticas

$$
\sum_{(\mu \nu \sigma)}\left(A^{\sigma k} \frac{\partial \delta^{\mu \nu}}{\partial q^{k}}-B_{\tau}^{\mu \nu} \delta^{\tau \sigma}\right)=0
$$


donde $\sum_{(\mu \nu \sigma)}$ representa la suma sobre permutaciones cíclicas únicamente. Notemos que hay una ecuación por cada elección de índices $\mu \nu \sigma$, de modo que hay

$$
\frac{(n-m+2)(n-m+1)(n-m)}{6} \text { ecuaciones. }
$$

Observación 3.4. 1. La asignación

$$
\mathbb{V} \mapsto\left(\delta, \gamma, \mathbb{V}_{\underline{m}}\right)
$$

es una aplicación suave con inversa suave. De hecho, dicha inversa está dada por

$$
\left\{\begin{array}{l}
\mathbb{V}^{s+a, s+b}=\mathbb{V}_{\underline{m}}^{a b} \\
\mathbb{V}^{\mu, s+a}=\mathbb{V}^{s+a, \mu}=\mathbb{V}_{\underline{\underline{m}}}^{a b} \gamma_{b}^{\mu} \\
\mathbb{V}^{\mu \nu}=\delta^{\mu \nu}+\gamma_{a}^{\mu} \mathbb{V}_{\underline{m}}^{a b} \gamma_{b}^{\mu}
\end{array} .\right.
$$

Más aun, $\mathbb{V}$ es simétrica y definida positiva si y sólo si $\delta$ y $\mathbb{V}_{\underline{m}}$ lo son. En efecto, si $\mathbb{V}$ es simétrica y definida positiva, también lo es $\mathbb{V}_{\underline{m}}$ gracias al Teorema A.2 y a la simetría de $\mathbb{V}$. A su vez, $\delta$ resulta simétrica por (3.8) y definida positiva por el Corolario A.1. Por otro lado, si $\delta$ y $\mathbb{V}_{\underline{m}}$ son simétricas y definidas positivas, $\mathbb{V}$ resulta simétrica por (3.13) y es definida positiva por el Teorema A.2 y el Corolario A.1 nuevamente.

2. Como era de esperar, la cantidad de ecuaciones cinéticas coincide con (2.42).

3. Vale la pena señalar que las variables $\gamma_{b}^{\mu}$ no aparecen derivadas en (3.12), de modo que en esta versión de las ecuaciones cinéticas podemos pensar en las variables $\delta^{\mu \nu}$ como las incógnitas y en las variables $\gamma_{b}^{\mu}$ como parámetros para dichas ecuaciones. Por otro lado, las variables $\left(\mathbb{V}_{\underline{m}}\right)_{a b} \mathrm{ni}$ siquiera aparecen en (3.12).

Observación 3.5. En el caso en que $m=1$ notemos que, usando una carta $(U, \varphi)$ adaptada a $W$ y tomando $W_{q}=\operatorname{span}\{\xi(q)\}$, se tiene que

$$
\operatorname{vlift}_{\alpha}^{\pi}(\xi(\pi(\alpha)))=\left.\frac{\partial}{\partial p_{n}}\right|_{\alpha}, \quad \forall \alpha \in T^{*} U
$$

Luego, la señal de control $Y$ sobre $T^{*} U$ se escribirá

$$
Y(\alpha)=\left.\lambda(\alpha) \frac{\partial}{\partial p_{n}}\right|_{\alpha},
$$

o bien en coordenadas

$$
Y(\mathbf{q}, \mathbf{p})=(0,0,0, \lambda(\mathbf{q}, \mathbf{p}))
$$

donde [recordar (1.38)]

$$
\lambda(\mathbf{q}, \mathbf{p})=-\frac{\mu(\mathbf{q}, \mathbf{p})+\{V, H\}(\mathbf{q}, \mathbf{p})}{\left(p_{n}+\gamma^{\mu}(\mathbf{q}) p_{\mu}\right) \mathbb{V}^{n n}(\mathbf{q})}
$$


A su vez, la versión local de (1.41) se escribirá

$$
\mu(\mathbf{q}, \mathbf{p})=\varkappa\left(p_{i} \mathbb{V}^{n i}(\mathbf{q})\right)^{2}=\varkappa\left(\gamma^{\mu}(\mathbf{q}) p_{\mu}+p_{n}\right)^{2}\left(\mathbb{V}^{n n}(\mathbf{q})\right)^{2}
$$

En el caso de las ecuaciones potenciales (1.45), podemos proceder análogamente y encontrar

$$
A^{\sigma k} \frac{\partial v}{\partial q^{k}}-\frac{\partial h}{\partial q^{\tau}} \delta^{\tau \sigma}=0
$$

que es un sistema de $s$ PDEs para $v$.

Observación 3.6. Notemos que, considerando al el conjunto de incógnitas $\left(\delta^{\mu \nu}, v\right)$, el sistema de PDEs dado por (3.12) y (3.15) es un sistema lineal de PDEs.

Resumiendo, en el caso en el cual $W$ es un subfibrado vectorial integrable de rango $m$ y considerando una carta coordenada adaptada a $W$, las ecuaciones cinéticas y potenciales (1.44) y (1.45) [junto con (3.5)] están dadas por (3.12) y (3.15), respectivamente. Para regresar de las variables $\delta^{\mu \nu}, \gamma_{b}^{\mu}$ y $\left(\mathbb{V}_{\underline{m}}\right)_{a b}$ a las variables originales $\mathbb{V}^{i j}$, podemos utilizar el cambio inverso descripto en la Observación 3.4.

\subsection{Estabilizabilidad de sistemas 1-subactuados}

Dado un sistema Hamiltoniano 1-subactuado $(H, \mathcal{W})$ sobre una variedad suave $Q$ de dimensión $n$ (i.e. el grado de actuación es $n-1$ ) con $H$ simple, y dado un punto crítico $\bar{\alpha}$ de su campo vectorial Hamiltoniano asociado $X_{H}$, en esta sección encontraremos condiciones necesarias y suficientes para asegurar que un tal sistema puede ser estabilizado en $\bar{\alpha}$ exhibiendo una función de Lyapunov simple para el CLMS correspondiente. Más aun, en el caso en que $n=2$, probaremos en la sección siguiente que tales condiciones son también suficientes para asegurar estabilizabilidad asintótica local. Demostraremos estos resultados usando el método LCB simple que introdujimos anteriormente. Para ello, utilizaremos las expresiones de las ecuaciones cinéticas y potenciales halladas en la sección anterior, i.e. las Ecs. (3.12) y (3.15).

\subsubsection{Las ecuaciones cinéticas y potenciales para sistemas 1 -subactuados}

Consideremos un sistema Hamiltoniano 1-subactuado $(H, \mathcal{W})$ sobre una $n$-variedad diferenciable $Q$, con $H$ simple y $\mathcal{W}$ dado por un subfibrado vectorial $W$ de $T^{*} Q$ de rango $m=n-1$. Debido a razones dimensionales, $(H, \mathcal{W})$ resulta regularmente actuado (i.e. $W$ es un subfibrado integrable). Luego, tomando una carta coordenada adaptada a $W,(U, \varphi)$, las ecuaciones cinéticas y potenciales para nuestro sistema están dadas por (3.12) y (3.15). En este caso, como $s=1$, podemos simplificar la notación 
empleada en las ecuaciones de la siguiente manera:

$$
\begin{aligned}
\delta & :=\delta^{11}=\mathbb{V}^{11}-\mathbb{V}^{1,1+a}\left(\mathbb{V}_{\underline{m}}\right)_{a b} \mathbb{V}^{1+b, 1} \\
\gamma_{b} & :=\gamma_{b}^{1}=\left(\mathbb{V}_{\underline{m}}\right)_{b a} \mathbb{V}^{1+a, 1} \\
B & :=B_{1}^{11}=\frac{\partial \mathbb{H}^{11}}{\partial q^{1}}-2 \frac{\partial \mathbb{H}^{1,1+b}}{\partial q^{1}} \gamma_{b}+\gamma_{a} \gamma_{b} \frac{\partial \mathbb{H}^{1+a, 1+b}}{\partial q^{1}} \\
A^{k} & :=A^{1 k}=\mathbb{H}^{1 k}-\mathbb{H}^{k, 1+a} \gamma_{a}
\end{aligned}
$$

por lo que (3.12) se escribe

$$
A^{k} \frac{\partial \delta}{\partial q^{k}}-B \delta=0, \quad \delta>0
$$

mientras que (3.15) se reduce a

$$
A^{k} \frac{\partial v}{\partial q^{k}}-\frac{\partial h}{\partial q^{1}} \delta=0
$$

Siguiendo el desarrollo de la sección anterior, debemos encontrar $m$ funciones $\gamma_{1}, \ldots, \gamma_{m}$ para las cuales exista una solución local $\delta$ de (3.20) que sea positiva en un entorno de $\mathbf{0}$ y fijar una matriz simétrica y definida positiva $\mathbb{V}_{\underline{m}}$ de dimensión $m=n-1$. En tal caso, el término cinético de la función de Lyapunov buscada se obtiene con el cambio [recordar (3.13)]

$$
\begin{aligned}
\mathbb{V}^{1+a, 1+b} & =\mathbb{V}_{\underline{m}}^{a b} \\
\mathbb{V}^{1,1+a} & =\mathbb{V}^{1+a, 1}=\mathbb{V}_{\underline{\underline{m}}}^{a b} \gamma_{b} \\
\mathbb{V}^{11} & =\delta+\gamma_{a} \mathbb{V}_{\underline{\underline{m}}}^{a b} \gamma_{b} .
\end{aligned}
$$

Adicionalmente, para las funciones $\gamma_{b}$ y $\delta$ halladas anteriormente, debemos resolver la Ec. (3.21) para $v$. Dicha solución debe cumplir localmente

$$
v(\mathbf{0})=0 \quad \mathrm{y} \quad v(\mathbf{q})>0
$$

para todo $\mathbf{q} \neq \mathbf{0}$.

Resumiendo, para sistemas 1-subactuados, la aplicación del método LCB simple se condensa en las Ecuaciones (3.20), (3.21) y (3.22).

\subsubsection{Una condición suficiente para la estabilizabilidad mediante el método LCB simple}

Continuemos ahora con el sistema 1 -subactuado $(H, \mathcal{W})$ en las coordenadas $(U, \varphi)$ adaptadas a $W$, el punto crítico $(\mathbf{0}, \mathbf{0})$ de $X_{H}$ y la notación de la subsección anterior. Si encontramos una solución de las Ecuaciones (3.20), (3.21) y (3.22), entonces, siguiendo los puntos de la Definición 2.5, podremos construir un campo vectorial $Y$ y una función de Lyapunov simple para $X_{H}+Y$ y $(\mathbf{0}, \mathbf{0})$ (al menos localmente alrededor de $(\mathbf{0}, \mathbf{0}))$. Esto significa que el sistema $(H, \mathcal{W})$ es estabilizable alrededor de $\bar{\alpha}=$ $0_{\bar{q}}$. Luego, la estabilizabilidad de $(H, \mathcal{W})$ alrededor de $\bar{\alpha}$ puede ser analizada estudiando el problema de existencia de soluciones $(\delta, \gamma, v)$ de las Ecuaciones (3.20), (3.21) y (3.22). 
Como estrategia general, podemos pensar en resolver la primera ecuación para $\delta$. Luego, reemplazando dicha solución en (3.21), resolver para $v$. Siguiendo esta idea, notemos que la primera es una ecuación en derivadas parciales de primer orden lineal con campo vectorial característico dado por $\left(A^{1}, \ldots, A^{n}\right)$, de modo que podemos usar el Método de Características para resolverlo. Para ello, debemos fijar una condición de borde sobre una subvariedad de codimensión 1 no característica que contenga a $\mathbf{0}$. Recordemos que una subvariedad $\Gamma \subset \mathbb{R}^{n}$ es no característica si para todo $\mathbf{q} \in \Gamma$ vale que $\mathbb{R}^{n} \equiv T_{\mathbf{q}} \mathbb{R}^{n}=T_{\mathbf{q}} \Gamma \oplus \operatorname{span}\{A(\mathbf{q})\}$, i.e. $A$ es "transversal" a $\Gamma$ en todo punto de la misma. Dado que buscamos soluciones locales, es suficiente con encontrar una subvariedad $\Gamma$ que sea no característica en un entorno de $\mathbf{0}$. Como $\mathbb{H}$ es definida positiva, es claro que $\mathbb{H}^{n n}(\mathbf{0})>0$ y por lo tanto si escogemos

$$
\gamma_{m}(\mathbf{0}) \neq \frac{\mathbb{H}^{1 n}(\mathbf{0})-\mathbb{H}^{n, 1+x}(\mathbf{0}) \gamma_{x}(\mathbf{0})}{\mathbb{H}^{n n}(\mathbf{0})}
$$

se sigue que [ver Ec. (3.19)]

$$
A^{n}(\mathbf{0})=\mathbb{H}^{1 n}(\mathbf{0})-\mathbb{H}^{n, 1+a}(\mathbf{0}) \gamma_{a}(\mathbf{0}) \neq 0
$$

Por lo tanto podemos considerar la siguiente subvariedad de $\mathbb{R}^{n}$ :

$$
\Gamma:=\left\{\mathbf{q} \in \mathbb{R}^{n} \mid q^{n}=0\right\} \cap \varphi(U),
$$

que es claramente no característica en $\mathbf{0}$ y por continuidad es no característica en un entorno de $\mathbf{0}$. Achicando $U$ de ser necesario, podemos suponer que $\Gamma$ es efectivamente no característica. De ahora en más, usaremos este proceso de achicamiento implícitamente para indicar que una condición válida en un subconjunto abierto de $U$ es válida de hecho en todo $U$.

Dado que precisamos que $\delta>0$, podemos imponer la condición de borde $\left.\delta\right|_{\Gamma}=\mathfrak{g}$, donde $\mathfrak{g}$ : $\mathbb{R}^{n-1} \rightarrow \mathbb{R}$ es una función tal que $\mathfrak{g}(0)>0$. En este caso, el Teorema de Características establece que existe una única solución $\delta$ de (3.20) tal que $\delta\left(q^{1}, \ldots, q^{n-1}, 0\right)=\mathfrak{g}\left(q^{1}, \ldots, q^{n-1}\right)$, que implica, por continuidad, que $\delta(\mathbf{q})>0$ alrededor de $\mathbf{0}$ y por lo tanto en todo $U$.

Continuemos ahora con la ecuación potencial (3.21). Se trata en este caso también de una ecuación en derivadas parciales de primer orden, cuyo campo vectorial característico es nuevamente $A$. Suponiendo que vale (3.23), $\Gamma$ resulta nuevamente una subvariedad no característica y podemos imponer la condición de borde $\left.v\right|_{\Gamma}=\mathfrak{f}$, donde $\mathfrak{f}: \mathbb{R}^{n-1} \rightarrow \mathbb{R}$ es una función suave tal que $\mathfrak{f}(\mathbf{0})=0$. Esta última condición implica que $v(\mathbf{0})=0$, que es la primera condición que precisa (3.22). La segunda parte de la condición de positividad para $v$ establece que $\mathbf{0}$ es un punto crítico aislado de $v$ en donde se alcanza un mínimo local, o equivalentemente, 0 es un punto crítico de $v$ y el Hessiano de $v$, $\operatorname{Hess}(v)$, es definido positivo en $\mathbf{0}$. Analicemos estas condiciones. Dado que $\mathbf{0}$ es un punto crítico de $h$ (ver Observación 2.7), i.e. $\left(\frac{\partial h}{\partial q^{1}}(\mathbf{0}), \ldots, \frac{\partial h}{\partial q^{n}}(\mathbf{0})\right)=\mathbf{0}$, se sigue de (3.21) que $v$ debe cumplir

$$
A^{k}(\mathbf{0}) \frac{\partial v}{\partial q^{k}}(\mathbf{0})=0
$$


Luego, como $A^{n}(\mathbf{0}) \neq 0$ [ver (3.23)], para que $\left(\frac{\partial v}{\partial q^{1}}(\mathbf{0}), \ldots, \frac{\partial v}{\partial q^{n}}(\mathbf{0})\right)=\mathbf{0}$ es suficiente que $\mathbf{0}$ sea crítico para $\mathfrak{f}$, i.e. $\left(\frac{\partial \mathfrak{f}}{\partial q^{1}}(\mathbf{0}), \ldots, \frac{\partial \mathfrak{f}}{\partial q^{n-1}}(\mathbf{0})\right)=(0, \ldots, 0)$. Por otro lado, siguiendo el criterio de Sylvester (ver el Teorema A.1), Hess $(v)$ es definido positivo en $\mathbf{0}$ si y sólo si

$$
\operatorname{Hess}(v)_{\bar{r}}(\mathbf{0})>0 \quad \forall r=1, \ldots, n .
$$

Para evaluar esta condición, debemos describir las entradas de la matriz $\operatorname{Hess}(v)(\mathbf{0})$. Dichas entradas están relacionadas con la ecuación (3.21) y con la condición de borde $\left.v\right|_{\Gamma}=\mathfrak{f}$. En efecto, por un lado es claro que

$$
\frac{\partial^{2} v}{\partial q^{a} \partial q^{b}}(\mathbf{0})=\frac{\partial^{2} \mathfrak{f}}{\partial q^{a} \partial q^{b}}(\mathbf{0})=\operatorname{Hess}(\mathfrak{f})_{a b}(\mathbf{0})
$$

mientras que, derivando la Ec. (3.21) respecto de $q^{a}$ y evaluando en $\mathbf{0}$ (recordar que $\mathbf{0}$ es crítico para $v$ y $h)$

de modo que

$$
A^{k}(\mathbf{0}) \frac{\partial^{2} v}{\partial q^{a} \partial q^{k}}(\mathbf{0})-\delta(\mathbf{0}) \frac{\partial^{2} h}{\partial q^{a} \partial q^{1}}(\mathbf{0})=0
$$

y por lo tanto

$$
A^{n}(\mathbf{0}) \frac{\partial^{2} v}{\partial q^{a} \partial q^{n}}(\mathbf{0})=\delta(\mathbf{0}) \frac{\partial^{2} h}{\partial q^{a} \partial q^{1}}(\mathbf{0})-A^{b}(\mathbf{0}) \operatorname{Hess}(\mathfrak{f})_{a b}(\mathbf{0})
$$

$$
\frac{\partial^{2} v}{\partial q^{a} \partial q^{n}}(\mathbf{0})=\frac{\delta(\mathbf{0})}{A^{n}(\mathbf{0})} \frac{\partial^{2} h}{\partial q^{a} \partial q^{1}}(\mathbf{0})-\frac{A^{b}(\mathbf{0})}{A^{n}(\mathbf{0})} \operatorname{Hess}(\mathfrak{f})_{a b}(\mathbf{0}) .
$$

Por último, derivando respecto de $q^{n}$, evaluando en 0 y despejando obtenemos

$$
\frac{\partial^{2} v}{\partial\left(q^{n}\right)^{2}}(\mathbf{0})=\frac{\delta(\mathbf{0})}{A^{n}(\mathbf{0})} \frac{\partial^{2} h}{\partial q^{n} \partial q^{1}}(\mathbf{0})-\frac{A^{a}(\mathbf{0})}{A^{n}(\mathbf{0})} \frac{\partial^{2} v}{\partial q^{n} \partial q^{a}}(\mathbf{0}),
$$

y usando (3.24) hallamos

$$
\begin{aligned}
\frac{\partial^{2} v}{\partial\left(q^{n}\right)^{2}}(\mathbf{0}) & =\frac{\delta(\mathbf{0})}{A^{n}(\mathbf{0})} \frac{\partial^{2} h}{\partial q^{n} \partial q^{1}}(\mathbf{0})-\frac{A^{a}(\mathbf{0})}{A^{n}(\mathbf{0})}\left[\frac{\delta(\mathbf{0})}{A^{n}(\mathbf{0})} \frac{\partial^{2} h}{\partial q^{a} \partial q^{1}}(\mathbf{0})-\frac{A^{b}(\mathbf{0})}{A^{n}(\mathbf{0})} \operatorname{Hess}(\mathfrak{f})_{a b}(\mathbf{0})\right] \\
& =\frac{1}{A^{n}(\mathbf{0})}\left[\delta \frac{\partial^{2} h}{\partial q^{n} \partial q^{1}}-\delta \frac{A^{a}}{A^{n}} \frac{\partial^{2} h}{\partial q^{1} \partial q^{a}}+\frac{A^{a} \operatorname{Hess}(\mathfrak{f})_{a b} A^{b}}{A^{n}}\right](\mathbf{0}) .
\end{aligned}
$$

Dado que Hess $(\mathfrak{f})_{\bar{r}}(\mathbf{0})=\operatorname{Hess}(v)_{\bar{r}}(\mathbf{0})$ para todo $r=1, \ldots, n-1$, una condición necesaria para que $\operatorname{Hess}(v)(\mathbf{0})$ sea definido positivo es que $\operatorname{Hess}(\mathfrak{f})(\mathbf{0})$ lo sea. El siguiente lema da condiciones necesarias y suficientes para que $\operatorname{Hess}(v)(\mathbf{0})$ sea definido positivo.

Lema 3.1. Dadas funciones $\gamma_{b}$ tales que $A^{n}(\mathbf{0}) \neq 0$ y una solución local y positiva $\delta$ de (3.20), existe $\mathfrak{f}$ tal que $\operatorname{Hess}(v)(\mathbf{0})$ es definido positivo si y sólo si

$$
A^{i}(\mathbf{0}) \frac{\partial^{2} h}{\partial q^{1} \partial q^{i}}(\mathbf{0})>0 .
$$

Demostración. Supongamos primero que existe una función $\mathfrak{f}$ tal que $\operatorname{Hess}(v)(\mathbf{0})$ es definido positivo. Luego, para todo vector $\vec{x} \in \mathbb{R}^{n}$ no nulo vale que

$$
x^{i} \operatorname{Hess}(v)_{i j}(\mathbf{0}) x^{j}>0 .
$$


En particular, considerando $\vec{x}=(\vec{y}, 1)$, donde $\vec{y} \in \mathbb{R}^{n-1}$ está dado por

$$
\vec{y}=\left(\frac{A^{1}(\mathbf{0})}{A^{n}(\mathbf{0})}, \ldots, \frac{A^{n-1}(\mathbf{0})}{A^{n}(\mathbf{0})}\right),
$$

y usando (3.24) y (3.25) encontramos que

$$
\begin{aligned}
0 & <y^{a} \operatorname{Hess}(\mathfrak{f})_{a b}(\mathbf{0}) y^{b}+2 y^{a} \frac{\partial^{2} v}{\partial q^{a} \partial q^{n}}(\mathbf{0})+\frac{\partial^{2} v}{\partial\left(q^{n}\right)^{2}}(\mathbf{0}) \\
& =\frac{A^{a}(\mathbf{0}) \operatorname{Hess}(\mathfrak{f})_{a b}(\mathbf{0}) A^{b}(\mathbf{0})}{\left[A^{n}(\mathbf{0})\right]^{2}}+2 \frac{A^{a}(\mathbf{0})}{\left[A^{n}(\mathbf{0})\right]^{2}}\left[\delta(\mathbf{0}) \frac{\partial^{2} h}{\partial q^{a} \partial q^{1}}(\mathbf{0})-A^{b}(\mathbf{0}) \operatorname{Hess}(\mathfrak{f})_{a b}(\mathbf{0})\right] \\
& +\frac{1}{\left[A^{n}(\mathbf{0})\right]^{2}}\left[A^{n} \delta \frac{\partial^{2} h}{\partial q^{n} \partial q^{1}}-A^{a} \delta \frac{\partial^{2} h}{\partial q^{1} \partial q^{a}}+A^{a} \operatorname{Hess}(\mathfrak{f})_{a b} A^{b}\right](\mathbf{0}) \\
& =\frac{\delta(\mathbf{0})}{\left[A^{n}(\mathbf{0})\right]^{2}}\left[A^{i}(\mathbf{0}) \frac{\partial^{2} h}{\partial q^{1} \partial q^{i}}(\mathbf{0})\right]
\end{aligned}
$$

pero como $\delta(\mathbf{0})>0 \mathrm{y}\left[A^{n}(\mathbf{0})\right]^{2}>0$, esto puede ocurrir únicamente si

$$
A^{i}(\mathbf{0}) \frac{\partial^{2} h}{\partial q^{1} \partial q^{i}}(\mathbf{0})>0
$$

Recíprocamente, supongamos que vale (3.26), fijemos $\mathbb{F} \in M_{n-1}(\mathbb{R})$ una matriz simétrica y definida positiva y consideremos

$$
\mathfrak{f}\left(q^{1}, \ldots, q^{n-1}\right)=\frac{1}{2} q^{a} \mathbb{F}_{a b} q^{b}
$$

de modo que

$$
\operatorname{Hess}(\mathfrak{f})(\mathbf{0})=\mathbb{F}
$$

Denotemos $\vec{w} \in \mathbb{R}^{n-1}$ al vector de componentes

$$
w_{a}=\frac{\partial^{2} v}{\partial q^{n} \partial q^{a}}(\mathbf{0})
$$

Si existe $a$ para el cual $A^{a}(\mathbf{0}) \neq 0$, entonces es fácil ver [recordar (3.24)] que $\mathbb{F}$ puede tomarse de modo que $\vec{w} \neq \mathbf{0}$. Si por el contrario tenemos que $A^{a}(\mathbf{0})=0$ para todo $a$ y $\vec{w}=\mathbf{0}$, tenemos que

$$
\frac{\partial^{2} v}{\partial\left(q^{n}\right)^{2}}(\mathbf{0})=\frac{\delta(\mathbf{0})}{A^{n}(\mathbf{0})} \frac{\partial^{2} h}{\partial q^{n} \partial q^{1}}(\mathbf{0})=\frac{\delta(\mathbf{0})}{\left[A^{n}(\mathbf{0})\right]^{2}} A^{n}(\mathbf{0}) \frac{\partial^{2} h}{\partial q^{n} \partial q^{1}}(\mathbf{0})=\frac{\delta(\mathbf{0})}{\left[A^{n}(\mathbf{0})\right]^{2}} A^{i}(\mathbf{0}) \frac{\partial^{2} h}{\partial q^{i} \partial q^{1}}(\mathbf{0})>0,
$$

y usando el Criterio de Sylvester es claro que $\operatorname{Hess}(v)(\mathbf{0})$ es definido positivo.

Supongamos ahora que $\vec{w} \neq \mathbf{0}$ y denotemos

$$
m_{\mathbb{F}}=\min _{\vec{y} \in \mathbb{R}^{n-1}} y^{a} \mathbb{F}_{a b}(\mathbf{0}) y^{b} .
$$

Es claro que $m_{\mathbb{F}}>0$ puesto que $\mathbb{F}$ es definida positiva (de hecho, es fácil ver que $m_{\mathbb{F}}$ es el mínimo autovalor de $\mathbb{F}$ ). Con esta elección para $\mathfrak{f}$, y tomando un vector $\vec{x}=(\vec{y}, z)$ con $\vec{y} \in \mathbb{R}^{n-1}$ y denotando 
por $\|\cdot\|_{E}$ la norma euclídea, tenemos

$$
\begin{aligned}
x^{i} \operatorname{Hess}(v)_{i j}(\mathbf{0}) x^{j} & =y^{a} \mathbb{F}_{a b}(\mathbf{0}) y^{b}+2 z \vec{y} \cdot \vec{w}+z^{2} \frac{\partial^{2} v}{\partial\left(q^{n}\right)^{2}}(\mathbf{0}) \\
& \geq m_{\mathbb{F}}\|\vec{y}\|_{E}^{2}+2 z \vec{y} \cdot \vec{w}+z^{2} \frac{\partial^{2} v}{\partial\left(q^{n}\right)^{2}}(\mathbf{0}) \\
& \geq m_{\mathbb{F}}\|\vec{y}\|_{E}^{2}-2|z|\|\vec{y}\|_{E}\|\vec{w}\|_{E}+z^{2} \frac{\partial^{2} v}{\partial\left(q^{n}\right)^{2}}(\mathbf{0}) \\
& =m_{\mathbb{F}}\left(\|\vec{y}\|_{E}^{2}-2 \frac{|z|}{\sqrt{m_{\mathbb{F}}}} \frac{\|\vec{w}\|_{E}}{\sqrt{m_{\mathbb{F}}}}\|\vec{y}\|_{E}+\frac{z^{2}}{m_{\mathbb{F}}} \frac{\partial^{2} v}{\partial\left(q^{n}\right)^{2}}(\mathbf{0})\right),
\end{aligned}
$$

y definiendo $\beta=\frac{\|\vec{w}\|_{E}}{\sqrt{m_{\mathbb{F}}}}$

$$
\begin{aligned}
x^{i} \operatorname{Hess}(v)_{i j}(\mathbf{0}) x^{j} & =m_{\mathbb{F}} \beta^{2}\left(\frac{\|\vec{y}\|_{E}^{2}}{\beta^{2}}-2 \frac{|z|}{\sqrt{m_{\mathbb{F}}}} \frac{\|\vec{y}\|_{E}}{\beta}+\frac{z^{2}}{m_{\mathbb{F}} \beta^{2}} \frac{\partial^{2} v}{\partial\left(q^{n}\right)^{2}}(\mathbf{0})\right) \\
& =m_{\mathbb{F}} \beta^{2}\left[\left(\frac{1}{\beta^{2}} \frac{\partial^{2} v}{\partial\left(q^{n}\right)^{2}}(\mathbf{0})-1\right) \frac{z^{2}}{m_{\mathbb{F}}}+\left(\frac{|z|}{\sqrt{m_{\mathbb{F}}}}-\frac{\|\vec{y}\|_{E}}{\beta}\right)^{2}\right] .
\end{aligned}
$$

Por lo tanto, es suficiente que $\frac{\partial^{2} v}{\partial\left(q^{n}\right)^{2}}(\mathbf{0})>\beta^{2}$ para que $\operatorname{Hess}(v)(\mathbf{0})$ sea definido positivo. Consideremos ahora $\mathbb{F}=m_{\mathbb{F}} \mathbb{I}$, donde $\mathbb{I}$ es la matriz identidad de dimensión $n-1$ y veamos que podemos escoger $m_{\mathbb{F}}$ para satisfacer esta desigualdad. Utilizando (3.25) y la definición de $\beta$ esta desigualdad es equivalente a (omitimos el símbolo $\mathbf{0}$ para no recargar la notación)

$$
\begin{aligned}
\frac{1}{\left(A^{n}\right)^{2}}\left[\delta A^{n} \frac{\partial^{2} h}{\partial q^{1} \partial q^{n}}-\delta A^{a} \frac{\partial^{2} h}{\partial q^{1} \partial q^{a}}+m_{\mathbb{F}}\|\vec{A}\|_{E}^{2}\right] & >\frac{1}{\left(A^{n}\right)^{2} m_{\mathbb{F}}}\left[\sum_{a=1}^{m} \delta^{2}\left(\frac{\partial^{2} h}{\partial q^{1} \partial q^{a}}\right)^{2}\right. \\
& \left.-2 m_{\mathbb{F}} \delta A^{a} \frac{\partial^{2} h}{\partial q^{1} \partial q^{a}}+m_{\mathbb{F}}^{2}\|\vec{A}\|_{E}^{2}\right]
\end{aligned}
$$

o bien, simplificando,

$$
\begin{gathered}
\delta A^{n} \frac{\partial^{2} h}{\partial q^{1} \partial q^{n}}+\delta A^{a} \frac{\partial^{2} h}{\partial q^{1} \partial q^{a}}>\frac{1}{m_{\mathbb{F}}} \sum_{a=1}^{m} \delta^{2}\left(\frac{\partial^{2} h}{\partial q^{1} \partial q^{a}}\right)^{2} \Leftrightarrow \\
\delta A^{i} \frac{\partial^{2} h}{\partial q^{1} \partial q^{i}}>\frac{1}{m_{\mathbb{F}}} \sum_{a=1}^{m} \delta^{2}\left(\frac{\partial^{2} h}{\partial q^{1} \partial q^{a}}\right)^{2} \Leftrightarrow \\
m_{\mathbb{F}}>\delta \frac{\sum_{a=1}^{m}\left(\frac{\partial^{2} h}{\partial q^{1} \partial q^{a}}\right)^{2}}{A^{i} \frac{\partial^{2} h}{\partial q^{1} \partial q^{i}}} .
\end{gathered}
$$

Por lo tanto, basta escoger $m_{\mathbb{F}}$ de modo tal que

$$
m_{\mathbb{F}}>\delta \frac{\sum_{a=1}^{m}\left(\frac{\partial^{2} h}{\partial q^{1} \partial q^{a}}\right)^{2}}{A^{i} \frac{\partial^{2} h}{\partial q^{1} \partial q^{i}}},
$$

para lograr que Hess $(v)(\mathbf{0})$ sea definido positivo (notar que esto siempre es posible). 
El lema anterior da condiciones necesarias y suficientes para lograr que la solución $v$ encontrada mediante el Método de Características presente un mínimo local aislado en $\mathbf{0}$. La condición 3.26 establece una relación entre los valores de las funciones $\mathbb{H}^{i j}$, $\operatorname{Hess}(h)_{1 i}$ y $\gamma_{b}$ en $\mathbf{0}$. Por esta razón, es razonable preguntarnos si es posible escoger las funciones $\gamma_{b}$ de modo que (3.26) se cumpla. El siguiente lema dice que esto no siempre es posible, sino que depende del sistema no actuado.

Lema 3.2. Existen constantes $\gamma_{a}(\mathbf{0})$ para las cuales $A^{i}(\mathbf{0}) \frac{\partial^{2} h}{\partial q^{1} \partial q^{i}}(\mathbf{0})>0$ si y sólo si

$$
\left\{\begin{array}{l}
\frac{\partial^{2} h}{\partial q^{1} \partial q^{i}}(\mathbf{0}) \mathbb{H}^{i, 1+a}(\mathbf{0}) \neq 0, \quad \text { paraalgún } \quad 1 \leq a \leq n-1, \quad \text { o bien } \\
\frac{\partial^{2} h}{\left(\partial q^{1}\right)^{2}}(\mathbf{0})>0
\end{array}\right.
$$

Másaun,

1. si $\frac{\partial^{2} h}{\partial q^{1} \partial q^{i}}(\mathbf{0}) \mathbb{H}^{i, 1+a}(\mathbf{0}) \neq 0$ basta tomar

$$
\left|\gamma_{a}(\mathbf{0})\right|>\left|\frac{\frac{\partial^{2} h}{\partial q^{1} \partial q^{i}}(\mathbf{0}) \mathbb{H}^{1 i}(\mathbf{0})-\sum_{\substack{x=1 \\ x \neq a}}^{n-1} \frac{\partial^{2} h}{\partial q^{1} \partial q^{i}}(\mathbf{0}) \mathbb{H}^{i, 1+x}(\mathbf{0}) \gamma_{x}(\mathbf{0})}{\frac{\partial^{2} h}{\partial q^{1} \partial q^{i}}(\mathbf{0}) \mathbb{H}^{i, 1+a}(\mathbf{0})}\right|
$$

$y$

$$
\frac{\partial^{2} h}{\partial q^{1} \partial q^{i}}(\mathbf{0}) \mathbb{H}^{i, 1+a}(\mathbf{0}) \gamma_{a}(\mathbf{0})<0,
$$

con $\gamma_{x}(\mathbf{0})$ arbitrarios para todo $x \neq a$.

2. Si por el contrario $\frac{\partial^{2} h}{\partial q^{1} \partial q^{i}}(\mathbf{0}) \mathbb{H}^{i, 1+a}(\mathbf{0})=0$ para todo a pero $\frac{\partial^{2} h}{\left(\partial q^{1}\right)^{2}}(\mathbf{0})>0$, cualquier elección para las constantes $\gamma_{a}(\mathbf{0})$ es buena.

Demostración. Veamos primero la condición necesaria. Probemos el contrarrecíproco, es decir, supongamos que

$$
\mathbb{H}^{1+a, i}(\mathbf{0}) \frac{\partial^{2} h}{\partial q^{1} \partial q^{i}}(\mathbf{0})=0, \quad \forall a=1, \ldots, n-1 ; \quad \mathrm{y} \quad \frac{\partial^{2} h}{\left(\partial q^{1}\right)^{2}}(\mathbf{0}) \leq 0,
$$

y mostremos entonces que $A^{i}(\mathbf{0}) \frac{\partial^{2} h}{\partial q^{1} \partial q^{i}}(\mathbf{0}) \leq 0$. En efecto

$$
\begin{aligned}
A^{i}(\mathbf{0}) \frac{\partial^{2} h}{\partial q^{1} \partial q^{i}}(\mathbf{0}) & =\left(\mathbb{H}^{1 i}-\mathbb{H}^{i, 1+a} \gamma_{a}\right)(\mathbf{0}) \frac{\partial^{2} h}{\partial q^{1} \partial q^{i}}(\mathbf{0})=\mathbb{H}^{1 i}(\mathbf{0}) \frac{\partial^{2} h}{\partial q^{1} \partial q^{i}}(\mathbf{0}) \\
& =\mathbb{H}^{11}(\mathbf{0}) \frac{\partial^{2} h}{\partial\left(q^{1}\right)^{2}}(\mathbf{0})+\mathbb{H}^{1,1+a}(\mathbf{0}) \frac{\partial^{2} h}{\partial q^{1} \partial q^{1+a}}(\mathbf{0}) .
\end{aligned}
$$

Ahora, dado que $\mathbb{H}(\mathbf{0})$ es definida positiva, sabemos que $\mathbb{H}_{\underline{m}}(\mathbf{0})$ es invertible y por lo tanto la identidad

$$
\mathbb{H}^{1+a, i}(\mathbf{0}) \frac{\partial^{2} h}{\partial q^{1} \partial q^{i}}(\mathbf{0})=0
$$

o equivalentemente

$$
\mathbb{H}_{\underline{m}}^{a b}(\mathbf{0}) \frac{\partial^{2} h}{\partial q^{1} \partial q^{1+b}}(\mathbf{0})=-\mathbb{H}^{1+a, 1}(\mathbf{0}) \frac{\partial^{2} h}{\partial\left(q^{1}\right)^{2}}(\mathbf{0}),
$$


implica que

$$
\frac{\partial^{2} h}{\partial q^{1} \partial q^{1+a}}(\mathbf{0})=-\left(\mathbb{H}_{\underline{m}}\right)_{a b}(\mathbf{0}) \mathbb{H}^{1+b, 1}(\mathbf{0}) \frac{\partial^{2} h}{\partial\left(q^{1}\right)^{2}}(\mathbf{0}),
$$

de modo que insertando esta última expresión en (3.29), encontramos

$$
\begin{aligned}
A^{i}(\mathbf{0}) \frac{\partial^{2} h}{\partial q^{1} \partial q^{i}}(\mathbf{0}) & =\left(\mathbb{H}^{11}(\mathbf{0})-\mathbb{H}^{1,1+a}(\mathbf{0})\left(\mathbb{H}_{\underline{m}}\right)_{a b}(\mathbf{0}) \mathbb{H}^{1+b, 1}(\mathbf{0})\right) \frac{\partial^{2} h}{\partial\left(q^{1}\right)^{2}}(\mathbf{0}) \\
& =\operatorname{det}(\delta(\mathbb{H}(\mathbf{0}), 1)) \frac{\partial^{2} h}{\partial\left(q^{1}\right)^{2}}(\mathbf{0})=\frac{\operatorname{det}\left(\mathbb{H}_{(}(\mathbf{0})\right)}{\operatorname{det}\left[\mathbb{H}_{\underline{m}}(\mathbf{0})\right]} \frac{\partial^{2} h}{\partial\left(q^{1}\right)^{2}}(\mathbf{0}) \leq 0,
\end{aligned}
$$

donde hemos usado la notación del Apéndice y el Lema A.2.

Probemos ahora la suficiencia. Dado que

$$
\begin{aligned}
A^{i}(\mathbf{0}) \frac{\partial^{2} h}{\partial q^{1} \partial q^{i}}(\mathbf{0}) & =\left(\mathbb{H}^{1 i}-\mathbb{H}^{i, 1+a} \gamma_{a}\right)(\mathbf{0}) \frac{\partial^{2} h}{\partial q^{1} \partial q^{i}}(\mathbf{0}) \\
& =\mathbb{H}^{1 i}(\mathbf{0}) \frac{\partial^{2} h}{\partial q^{1} \partial q^{i}}(\mathbf{0})-\mathbb{H}^{i, 1+a}(\mathbf{0}) \gamma_{a}(\mathbf{0}) \frac{\partial^{2} h}{\partial q^{1} \partial q^{i}}(\mathbf{0})
\end{aligned}
$$

lo que debemos probar es que existen constantes $\gamma_{a}(\mathbf{0})$ tales que

$$
\frac{\partial^{2} h}{\partial q^{1} \partial q^{i}}(\mathbf{0}) \mathbb{H}^{i, 1+a}(\mathbf{0}) \gamma_{a}(\mathbf{0})<\frac{\partial^{2} h}{\partial q^{1} \partial q^{i}}(\mathbf{0}) \mathbb{H}^{1 i}(\mathbf{0}) .
$$

Debido a que el término de la izquierda es lineal en $\gamma_{a}(\mathbf{0})$, basta ver que existe al menos un $a$ para el que

$$
\frac{\partial^{2} h}{\partial q^{1} \partial q^{i}}(\mathbf{0}) \mathbb{H}^{i, 1+a}(\mathbf{0}) \neq 0 .
$$

Pero esta es exactamente la primera de las hipótesis del Lema. En este caso, podemos tomar

$$
\left|\gamma_{a}(\mathbf{0})\right|>\left|\frac{\frac{\partial^{2} h}{\partial q^{1} \partial q^{i}}(\mathbf{0}) \mathbb{H}^{1 i}(\mathbf{0})-\sum_{\substack{x=1 \\ x \neq a}}^{n-1} \frac{\partial^{2} h}{\partial q^{1} \partial q^{i}}(\mathbf{0}) \mathbb{H}^{i, 1+x}(\mathbf{0}) \gamma_{x}(\mathbf{0})}{\frac{\partial^{2} h}{\partial q^{1} \partial q^{i}}(\mathbf{0}) \mathbb{H}^{i, 1+a}(\mathbf{0})}\right|
$$

y tal que

Supongamos ahora que

$$
\frac{\partial^{2} h}{\partial q^{1} \partial q^{i}}(\mathbf{0}) \mathbb{H}^{i, 1+a}(\mathbf{0}) \gamma_{a}(\mathbf{0})<0
$$

$$
\frac{\partial^{2} h}{\partial\left(q^{1}\right)^{2}}(\mathbf{0})>0 \quad \text { y } \quad \frac{\partial^{2} h}{\partial q^{1} \partial q^{i}}(\mathbf{0}) \mathbb{H}^{i, 1+a}(\mathbf{0})=0 \quad \forall a=1, \ldots, n-1 .
$$

En este caso, el término izquierdo de (3.30) es 0 . Usando la positividad de $\mathbb{H}^{i j}(\mathbf{0})$, tenemos

$$
\begin{aligned}
0 & <\frac{\partial^{2} h}{\partial q^{1} \partial q^{i}}(\mathbf{0}) \mathbb{H}^{i j}(\mathbf{0}) \frac{\partial^{2} h}{\partial q^{1} \partial q^{j}}(\mathbf{0}) \\
& =\frac{\partial^{2} h}{\partial q^{1} \partial q^{i}}(\mathbf{0})\left(\mathbb{H}^{i 1}(\mathbf{0}) \frac{\partial^{2} h}{\partial\left(q^{1}\right)^{2}}(\mathbf{0})+\mathbb{H}^{i, 1+a}(\mathbf{0}) \frac{\partial^{2} h}{\partial q^{1} \partial q^{1+a}}(\mathbf{0})\right) \\
& =\frac{\partial^{2} h}{\partial q^{1} \partial q^{i}}(\mathbf{0}) \mathbb{H}^{i 1}(\mathbf{0}) \frac{\partial^{2} h}{\partial\left(q^{1}\right)^{2}}(\mathbf{0})
\end{aligned}
$$


y como $\frac{\partial^{2} h}{\partial\left(q^{1}\right)^{2}}(\mathbf{0})>0$, deducimos que

$$
\frac{\partial^{2} h}{\partial q^{1} \partial q^{i}}(\mathbf{0}) \mathbb{H}^{i 1}(\mathbf{0})>0
$$

que es lo que queríamos probar [ver (3.30)].

Resumiendo, si la condición (3.27) se cumple, para encontrar una solución local $\left(\delta, \gamma_{a}, v\right)$ de (3.20), (3.21) y (3.22), es suficiente escoger las constantes $\gamma_{a}(\mathbf{0})$ cumpliendo (3.23) y siguiendo los puntos 1 y 2 del Lema 3.2, y las funciones $\mathfrak{g}$ y $\mathfrak{f}$ (que definen las condiciones de borde de las ecuaciones cinéticas y potenciales) de manera tal que

$$
\mathfrak{g}(\mathbf{0})>0, \quad \mathfrak{f}(\mathbf{0})=0, \quad \nabla \mathfrak{f}(\mathbf{0})=\mathbf{0},
$$

y

$$
m_{\mathbb{F}}=\min _{\vec{y} \in \mathbb{R}^{n-1}} y^{a} \operatorname{Hess}(\mathfrak{f})_{a b}(\mathbf{0}) y^{b}>\left[\delta \frac{\sum_{a=1}^{m} \delta^{2}\left(\frac{\partial^{2} h}{\partial q^{1} \partial q^{a}}\right)^{2}}{A^{i} \frac{\partial^{2} h}{\partial q^{1} \partial q^{i}}}\right](\mathbf{0}) .
$$

En consecuencia, hemos probado lo siguiente.

Teorema 3.1. Consideremos un sistema subactuado $(H, \mathcal{W})$ sobre una n-variedad $Q$, con $H$ simple y $\mathcal{W}$ dado por un subfibrado $W$ de rango $1, y$ consideremos un punto crítico $\bar{\alpha}=0_{\bar{q}} \in T_{\bar{q}}^{*} Q$ de $X_{H}$. Fijemos una carta coordenada adaptada a $W$ y centrada en $\bar{q}$, y consideremos la correspondiente expresión local de $H$ dada por (3.3). Si la Ec. (3.27) se cumple, entonces $(H, \mathcal{W})$ es estabilizable en $\bar{\alpha}$, i.e. existe un campo vectorial local $Y$, definido en un entorno de $\bar{\alpha}$, tal que el sistema a lazo cerrado $(H, Y)_{\mathcal{W}}$ resulta estable en $\bar{\alpha}$. Más aun, dicha estabilidad puede asegurarse mediante la existencia de una función de Lyapunov simple.

Un resultado análogo al del teorema anterior fue encontrado en [13], pero en el contexto del energy shaping.

Observación 3.7. En la referencia [13], se probó que una condición suficiente (intrínseca) para alcanzar la estabilidad de puntos de equilibrio inestables en sistemas 1-subactuados es que

1. la linealización del sistema en $\bar{\alpha}$ es controlable o bien,

2. la linealización del sistema en $\bar{\alpha}$ no es controlable pero sus modos no controlables forman un par imaginario puro.

De hecho, usando la condición de controlabilidad para sistemas lineales [9], no es difícil ver que en una carta coordenada adaptada a $W$ centrada en $\bar{q}$, la primera de estas condiciones es equivalente a

$$
\frac{\partial^{2} h}{\partial q^{1} \partial q^{i}}(\mathbf{0}) \mathbb{H}^{i, 1+a}(\mathbf{0}) \neq 0, \quad \text { para algún } \quad 1 \leq a \leq n-1,
$$


mientras que la segunda es equivalente a

$$
\frac{\partial^{2} h}{\partial q^{1} \partial q^{i}}(\mathbf{0}) \mathbb{H}^{i, 1+a}(\mathbf{0})=0, \quad \text { para todo } \quad 1 \leq a \leq n-1, \quad \text { y } \quad \frac{\partial^{2} h}{\left(\partial q^{1}\right)^{2}}(\mathbf{0})>0 .
$$

Definición 3.2. Dado un sistema subactuado $(H, \mathcal{W})$ con un punto de equilibrio inestable $\bar{\alpha}$, diremos que el sistema es tipo 1 si la linealización del sistema en $\bar{\alpha}$ es controlable y que es tipo 2 si dicha linealización es no controlable con modos no controlables dados por un par imaginario puro.

\subsubsection{Una condición necesaria para la existencia de una función de Lyapunov simple}

Fijemos nuevamente un sistema 1 -subactuado sobre una $n$-variedad suave $Q$ y consideremos un punto de equilibrio $\bar{\alpha}=0_{\bar{q}} \in T_{\bar{q}}^{*} Q$ de $X_{H}$. Supongamos que $(H, \mathcal{W})$ puede ser estabilizado en $\bar{\alpha}$, y que la estabilidad del sistema a lazo cerrado correspondiente puede ser asegurada por la existencia de una función de Lyapunov simple. Más precisamente, supongamos que existe un campo vectorial $Y$ con imagen en $\mathcal{W}$ y una función de Lyapunov simple $V$ para $X_{H}+Y$ y $\bar{\alpha}$, definida al menos en un entorno de $\bar{\alpha}$. Entonces, el Teorema 2.3 nos dice que $Y$ y $V$ deben estar dadas por el método LCB simple. En particular, $V$ está dada localmente, en una carta $(U, \varphi)$ adaptada a $W$ y centrada en $\bar{q}$, por una solución $\left(\delta, \gamma_{a}, v\right)$ de las Ecuaciones (3.20), (3.21) y (3.22), y por (3.4), (3.16) y (3.17). Queremos mostrar entonces que se cumple (3.27). Para ello, supongamos primero que $A^{n}(\mathbf{0}) \neq 0$ [i.e. $\gamma_{m}(\mathbf{0})$ satisface (3.23)] y definamos $\mathfrak{f}\left(q^{1}, \ldots, q^{n-1}\right):=v\left(q^{1}, \ldots, q^{n-1}, 0\right)$, para todo $\left(q^{1}, \ldots, q^{n-1}\right) \in \mathbb{R}^{n-1}$ tal que $\left(q^{1}, \ldots, q^{n-1}, 0\right) \in \varphi(U)$. Como hicimos en la subsección anterior, derivando (3.21) y evaluando el resultado en $\mathbf{0}$ (y usando además que $\mathbf{0}$ es crítico para $h$ ), llegamos nuevamente a las Ecuaciones (3.24) y (3.25). Luego, usando la positividad de $v$, vemos que (3.26) es una condición necesaria (ver detalles en la Demostración del Lema 3.1). Pero de acuerdo al Lema 3.2, esta condición es equivalente a (3.27). Si $A^{n}(\mathbf{0})=0$ pero existe $a$ tal que $A^{1+a}(\mathbf{0}) \neq 0$, podemos efectuar una permutación de coordenadas $q^{1+a} \leftrightarrow q^{n}$ y proceder como explicamos anteriormente (notemos en este caso que las nuevas coordenadas son también adaptadas a $W$ ).

Supongamos ahora que $A^{1+a}(\mathbf{0})=0$ para todo $a$. Luego, usando (3.19), tenemos que

$$
\mathbb{H}^{1,1+a}(\mathbf{0})-\mathbb{H}^{1+a, 1+b}(\mathbf{0}) \gamma_{b}(\mathbf{0})=0,
$$

y sumando $\mathbb{H}^{1+a, 1+b}(\mathbf{0}) \gamma_{b}(\mathbf{0})$ en ambos miembros,

$$
\mathbb{H}_{\underline{m}}^{a b}(\mathbf{0}) \gamma_{b}(\mathbf{0})=\mathbb{H}^{1,1+a}(\mathbf{0})
$$

Por último, como $\mathbb{H}^{1+a, 1+b}(\mathbf{0})$ es invertible,

$$
\gamma_{b}(\mathbf{0})=\left(\mathbb{H}_{\underline{m}}\right)_{b a}(\mathbf{0}) \mathbb{H}^{1,1+a}(\mathbf{0})
$$


Luego

$$
\begin{aligned}
A^{1}(\mathbf{0}) & =\mathbb{H}^{11}(\mathbf{0})-\mathbb{H}^{1,1+b}(\mathbf{0}) \gamma_{b}(\mathbf{0})=\mathbb{H}^{11}(\mathbf{0})-\mathbb{H}^{1,1+b}(\mathbf{0})\left(\mathbb{H}_{\underline{m}}\right)_{b a}(\mathbf{0}) \mathbb{H}^{1,1+a}(\mathbf{0}) \\
& =\operatorname{det}[\delta(\mathbb{H}(\mathbf{0}), 1)]=\frac{\operatorname{det} \mathbb{H}(\mathbf{0})}{\operatorname{det}\left(\mathbb{H}_{\underline{m}}\right)}>0 .
\end{aligned}
$$

Derivando (3.21) respecto de $q^{1}$ y evaluando en $\mathbf{0}$ (recordar que $\mathbf{0}$ es crítico para $h$ y $v$ ), se sigue que

$$
A^{1}(\mathbf{0}) \frac{\partial^{2} v}{\partial\left(q^{1}\right)^{2}}(\mathbf{0})=\delta(\mathbf{0}) \frac{\partial^{2} h}{\partial\left(q^{1}\right)^{2}}(\mathbf{0}),
$$

y dado que $\operatorname{Hess}(v)(\mathbf{0})$ es definido positivo y $\delta(\mathbf{0})$ y $A^{1}(\mathbf{0})$ son positivos, deducimos que

$$
\frac{\partial^{2} h}{\partial\left(q^{1}\right)^{2}}(\mathbf{0})>0
$$

En otras palabras, nuevamente la condición (3.27) debe cumplirse. Combinando estos resultados junto con el Teorema 3.1, obtenemos la siguiente equivalencia.

Teorema 3.2. Consideremos un sistema 1-subactuado $(H, \mathcal{W})$ sobre una $n$-variedad y consideremos un punto crítico $\bar{\alpha}=0_{\bar{q}} \in T_{\bar{q}}^{*} Q$ de $X_{H}$. Entonces, $(H, \mathcal{W})$ es estabilizable en $\bar{\alpha}$, de manera tal que dicha estabilidad puede demostrarse mediante la existencia de una función de Lyapunov simple, ${ }^{3}$ si y sólo si en una carta coordenada adaptada a $W$ y centrada en $\bar{q}$, la función $H$ cumple

$$
\left\{\begin{array}{l}
\frac{\partial^{2} h}{\partial q^{1} \partial q^{i}}(\mathbf{0}) \mathbb{H}^{i, 1+a}(\mathbf{0}) \neq 0, \quad \text { para algún } \quad 1 \leq a \leq n-1, \quad \text { o bien } \\
\frac{\partial^{2} h}{\left(\partial q^{1}\right)^{2}}(\mathbf{0})>0,
\end{array}\right.
$$

donde estamos usando la expresión local de $H$ dada por (3.3).

A continuación reproduciremos todos estos resultados en el caso particular de sistemas con dos grados de libertad, lo cual será de suma utilidad en la Sección \$3.3.

\subsubsection{Sistemas con dos grados de libertad}

Supongamos que $Q$ es una 2 -variedad suave y sea $(H, \mathcal{W})$ un sistema subactuado con $H$ simple y $\mathcal{W}$ dado por un subfibrado $W$ de $T^{*} Q$. Por razones dimensionales el sistema es regularmente actuado. $\mathrm{Si}(U, \varphi)$ es una carta adaptada a $W$, denotaremos las coordenada de la siguiente manera

$$
x:=q^{1}, \quad y:=q^{2}, \quad p_{x}:=p_{1}, \quad p_{y}:=p_{2},
$$

y las funciones simples $H$ y $V$ siguiendo (3.3) y (3.4) y adoptando la escritura

$$
\mathbb{H}(x, y)=:\left[\begin{array}{ll}
a(x, y) & b(x, y) \\
b(x, y) & c(x, y)
\end{array}\right], \quad \mathbb{V}(x, y)=:\left[\begin{array}{ll}
f(x, y) & g(x, y) \\
g(x, y) & l(x, y)
\end{array}\right],
$$

para las representaciones matriciales de los términos cinéticos. A continuación reproduciremos las expresiones de la sección anterior usando esta nueva notación:

\footnotetext{
${ }^{3}$ Vale la pena recordar que la existencia de una función de Lyapunov $V$ para un campo $X$ y un punto crítico $\bar{\alpha}$ es una condición suficiente para que $\bar{\alpha}$ sea estable pero no necesaria. En otras palabras, existen campos $X$ con puntos críticos estables para los cuales no es posible exhibir una función de Lyapunov.
} 
- dado que $\mathbb{H}$ es definida positiva, tenemos que $a(x, y), c(x, y)>0$ y $\Delta(x, y):=a(x, y) c(x, y)$ $b^{2}(x, y)>0$;

- dado que $\mathbb{V}$ es definida positiva, tenemos que

$$
f(x, y), l(x, y)>0 \quad \text { y } \quad f(x, y) l(x, y)-g^{2}(x, y)>0
$$

- siguiendo (3.16) y (3.17), definimos

$$
\delta:=\frac{f l-g^{2}}{l}, \quad \gamma:=\gamma_{1}=\frac{g}{l} ;
$$

- de (3.19) y (3.18), tenemos que

$$
A=\left(A^{1}, A^{2}\right)=(a-b \gamma, b-c \gamma), \quad B=a_{x}-2 \gamma b_{x}+\gamma^{2} c_{x} .
$$

- La ecuación cinética (3.20) junto con su condición de positividad (3.34) se escriben (de ahora en adelante, los subíndices $x$ e $y$ denotan derivación parcial)

$$
(a-b \gamma) \delta_{x}+(b-c \gamma) \delta_{y}=B \delta ; \quad \delta>0
$$

- la ecuación potencial (3.21) se reduce a

$$
(a-b \gamma) v_{x}+(b-c \gamma) v_{y}=h_{x} \delta
$$

- y las condiciones de positividad para $v$ se escriben

$$
v(0,0)=0, \quad v(x, y)>0 \quad \text { para todo } \quad(x, y) \neq(0,0) .
$$

- Las Ecuaciones (3.24) y (3.25) toman la forma

$$
\begin{aligned}
v_{x y}(\mathbf{0}) & =\frac{h_{x x}(\mathbf{0}) \mathfrak{g}(0)-(a-b \gamma)(\mathbf{0}) \mathfrak{f}^{\prime \prime}(0)}{(b-c \gamma)(\mathbf{0})} \\
v_{y y}(\mathbf{0}) & =\frac{h_{x y}(\mathbf{0}) \mathfrak{g}(0)(b-c \gamma)(\mathbf{0})-(a-b \gamma)(\mathbf{0}) h_{x x}(\mathbf{0}) \mathfrak{g}(0)+(a-b \gamma)^{2}(\mathbf{0}) \mathfrak{f}^{\prime \prime}(0)}{(b-c \gamma)^{2}(\mathbf{0})} .
\end{aligned}
$$

- La subvariedad característica $\Gamma$ es el eje $x$ y la condición de que $A$ sea no característico en un entorno a $\mathbf{0}$ es

$$
\gamma(\mathbf{0}) \neq \frac{b(\mathbf{0})}{c(\mathbf{0})}
$$

- La condición (3.26) se escribe

$$
\left[(a-b \gamma) h_{x x}+(b-c \gamma) h_{x y}\right](\mathbf{0})>0
$$


- y la condición (3.31) es

$$
\left[b h_{x x}+c h_{x y}\right](\mathbf{0}) \neq 0 \text { obien } \quad h_{x x}(\mathbf{0})>0 .
$$

- Las condiciones de borde son funciones de una única variable y deben cumplir

$$
\mathfrak{g}(0)>0, \quad \mathfrak{f}(0)=0, \quad \mathfrak{f}^{\prime}(0)=0,
$$

y

$$
\mathfrak{f}^{\prime \prime}(0)>\frac{h_{x x}^{2}(\mathbf{0}) \mathfrak{g}(0)}{\left[(a-b \gamma) h_{x x}+(b-c \gamma) h_{x y}\right](\mathbf{0})},
$$

donde $\mathfrak{f}^{\prime}$ y $\mathfrak{f}^{\prime \prime}$ son la primera y segunda derivadas de $\mathfrak{f}$.

En la Referencia [13], se prueba que la condición ${ }^{4}\left[b h_{x x}+c h_{x y}\right](\mathbf{0}) \neq 0$ también implica estabilizabilidad asintótica local (como se afirma sin demostración en [25]). En cualquier otro caso, la misma referencia presenta una condición adicional que es suficiente para asegurar la estabilizabilidad asintótica local. En la sección siguiente demostraremos que no es necesaria ninguna condición adicional, más que (3.42), para asegurar este tipo de estabilización.

\subsection{Estabilizabilidad asintótica local}

\subsubsection{Sistemas con dos grados de libertad}

Sea $(\delta, \gamma, v)$ una solución de (3.37), (3.38) y (3.39), con $\gamma$ cumpliendo (3.41) y con condiciones de borde dadas por $\mathfrak{g}$ y $\mathfrak{f}$, siguiendo la notación de la sección anterior. Es decir, $\delta$ y $v$ deben cumplir

$$
\delta(x, 0)=\mathfrak{g}(x) \quad \text { y } \quad v(x, 0)=\mathfrak{f}(x),
$$

siendo $\mathfrak{g}$ y $\mathfrak{f}$ funciones que satisfacen (3.43) y (3.44). Sea $V$ dada por (3.4), (3.33) y (3.35), i.e.

$$
V\left(x, y, p_{x}, p_{y}\right)=\left(p_{x}^{2}\left(\frac{\delta(x, y)}{l(x, y)}-\gamma^{2}(x, y)\right)+2 \gamma(x, y) p_{x} p_{y}+p_{y}^{2}\right) l(x, y)+v(x, y),
$$

para alguna función positiva $l$. Fijemos $\mu$ como en la Ec. (3.14). Usando (3.32) y (3.35), esto significa que

$$
\mu\left(x, y, p_{x}, p_{y}\right)=\varkappa\left(\gamma(x, y) p_{x}+p_{y}\right)^{2} l^{2}(x, y) .
$$

En este contexto y siguiendo la Observación 3.5, la señal de control que provee el método LCB simple se escribe

$$
Y(\alpha)=\left.\lambda(\alpha) \frac{\partial}{\partial p_{y}}\right|_{\alpha}, \forall \alpha \in T^{*} U,
$$

donde la expresión en coordenadas adaptadas para $\lambda$ está dada por

$$
\lambda\left(x, y, p_{x}, p_{y}\right)=-\varkappa\left(\gamma(x, y) p_{x}+p_{y}\right) l(x, y)-\frac{\{V, H\}\left(x, y, p_{x}, p_{y}\right)}{\left(\gamma(x, y) p_{x}+p_{y}\right) l(x, y)} .
$$

\footnotetext{
${ }^{4}$ De hecho, en esa misma referencia se considera una condición aun más débil (ver Teorema III.3).
} 
De acuerdo con los Teoremas 2.2, 2.3 y el Lema 2.2, la función $V$ definida más arriba es una función de Lyapunov para $X:=\left.X_{H}\right|_{T^{*} U}+Y$ y $\bar{\alpha}=0_{\bar{q}}$, con subvariedad de LaSalle [ver (1.28)]

$$
\mu^{-1}(0)=\varphi^{*}\left(\left\{\left(x, y, p_{x}, p_{y}\right): p_{x} \gamma(x, y)+p_{y}=0\right\}\right) \subset T^{*} U
$$

En lo que sigue probaremos que, sin necesidad de condiciones adicionales [más que la Ec. (3.42)], es posible escoger las condiciones de borde $\mathfrak{g}$ y $\mathfrak{f}$ [ver (3.45)], junto con un conjunto abierto $T \subset T^{*} U$ que contiene a $\bar{\alpha}$, de manera tal que la mayor subvariedad de $\mathcal{S}_{0}:=\mu^{-1}(0) \cap T$ que es $X$-invariante (ver Definición 1.5) es el conjunto unitario $\{\bar{\alpha}\}$. Esto implicaría, via el principio de invariancia de LaSalle, que $\bar{\alpha}$ es (localmente) asintóticamente estable para el sistema a lazo cerrado correspondiente. La demostración sigue el algoritmo presentado en el Lema 1.1.

Más concretamente, probaremos que:

Lema 3.3. Existen condiciones de borde $\mathfrak{g} y \mathfrak{f}$, una función $\gamma y$ un subconjunto abierto $T \ni \bar{\alpha} \operatorname{de} T^{*} U$, tal que [ver (1.29)]:

- el subconjunto $\mathcal{S}_{1}$ correspondiente a $\mathcal{S}_{0}=\mu^{-1}(0) \cap T$ es una subvariedad de $\mathcal{S}_{0}$;

- $\mathcal{S}_{2}$ es una subvariedad de $\mathcal{S}_{1}$

- $\mathcal{S}_{3}=\{\bar{\alpha}\}$.

Es suficiente tomar $\mathfrak{g} y \mathfrak{f}$ cumpliendo (3.43) y (3.44) y tales que

$$
\frac{\mathfrak{g}^{\prime}(0)}{\mathfrak{g}(0)} \neq-\left[\frac{2(b-c \gamma)}{\Delta}\left(b_{x}-\gamma c_{x}-\frac{B c}{2(b-c \gamma)}\right)\right](\mathbf{0}),
$$

escoger $\gamma(\mathbf{0})$ de acuerdo con (3.41), siguiendo los puntos 1 y 2 del Lema 3.2 y cumpliendo la condición adicional

$$
\gamma(\mathbf{0}) \neq \frac{(a(\mathbf{0}), b(\mathbf{0})) \mathbb{M}(\mathbf{0})\left(\begin{array}{l}
b(\mathbf{0}) \\
c(\mathbf{0})
\end{array}\right)}{(b(\mathbf{0}), c(\mathbf{0})) \mathbb{M}(\mathbf{0})\left(\begin{array}{l}
b(\mathbf{0}) \\
c(\mathbf{0})
\end{array}\right)}
$$

donde

$$
\mathbb{M}=\left[\begin{array}{cc}
\mathfrak{f}^{\prime \prime}(0) & \frac{h_{x x} \mathfrak{g}(0)-(a-b \gamma) \mathfrak{f}^{\prime \prime}(0)}{(b-c \gamma)} \\
\frac{h_{x x} \mathfrak{g}(0)-(a-b \gamma) \mathfrak{f}^{\prime \prime}(0)}{(b-c \gamma)} & \frac{h_{x y} \mathfrak{g}(0)(b-c \gamma)-(a-b \gamma) h_{x x} \mathfrak{g}(0)+(a-b \gamma)^{2} \mathfrak{f}^{\prime \prime}(0)}{(b-c \gamma)^{2}}
\end{array}\right] .
$$

Demostración. De acuerdo con (3.49), $\varphi^{*-1}\left(\mu^{-1}(0)\right)$ puede describirse como el conjunto de ceros de la submersión

$$
\mathfrak{F}\left(x, y, p_{x}, p_{y}\right)=\gamma(x, y) p_{x}+p_{y} .
$$

De ahora en más y para no recargar la notación, omitiremos la carta coordenada $\varphi$, i.e. identificaremos $U \operatorname{con} \varphi(U)$. Esto significa que $\bar{q}=\mathbf{0}$ y $\bar{\alpha}=(\mathbf{0}, \mathbf{0})$. Procederemos en tres pasos. 
1. Consideremos el conjunto $Z_{1} \subset \mu^{-1}(0)$ tal que $\mathfrak{F}_{*}(X)\left(x, y, p_{x}, p_{y}\right)=0$, donde [ver (3.47)]

$$
X=X_{H}+Y=\frac{\partial H}{\partial p_{x}} \frac{\partial}{\partial x}+\frac{\partial H}{\partial p_{y}} \frac{\partial}{\partial y}-\frac{\partial H}{\partial x} \frac{\partial}{\partial p_{x}}-\left(\frac{\partial H}{\partial y}-\lambda\right) \frac{\partial}{\partial p_{y}} .
$$

Por lo tanto, $Z_{1}$ está dado por

$$
\frac{\partial H}{\partial p_{x}} \frac{\partial \mathfrak{F}}{\partial x}+\frac{\partial H}{\partial p_{y}} \frac{\partial \mathfrak{F}}{\partial y}-\frac{\partial H}{\partial x} \frac{\partial \mathfrak{F}}{\partial p_{x}}-\left(\frac{\partial H}{\partial y}-\lambda\right) \frac{\partial \mathfrak{F}}{\partial p_{y}}=0
$$

o equivalentemente por

$$
\frac{\partial H}{\partial p_{x}} \gamma_{x} p_{x}+\frac{\partial H}{\partial p_{y}} \gamma_{y} p_{x}-\frac{\partial H}{\partial x} \gamma-\frac{\partial H}{\partial y}+\lambda=0
$$

у $p_{y}=-\gamma(x, y) p_{x}$. Usando (3.3) y (3.33), es sencillo ver que, sobre $\mu^{-1}(0)$,

$$
\frac{\partial H}{\partial x}=\frac{1}{2} B p_{x}^{2}+h_{x}, \quad \frac{\partial H}{\partial y}=\frac{1}{2} C p_{x}^{2}+h_{y}, \quad \frac{\partial H}{\partial p_{x}}=(a-b \gamma) p_{x} \quad \text { y } \quad \frac{\partial H}{\partial p_{y}}=(b-c \gamma) p_{x},
$$

donde

$$
B=a_{x}-2 \gamma b_{x}+\gamma^{2} c_{x} \quad \text { y } \quad C=a_{y}-2 \gamma b_{y}+\gamma^{2} c_{y} .
$$

Por otra parte los valores de la función $\lambda$ sobre el conjunto $\mu^{-1}(0)$, de acuerdo con (3.48), están dados por el límite

$$
\begin{aligned}
\lambda\left(x, y, p_{x},-\gamma(x, y) p_{x}\right) & =-\lim _{p_{y} \rightarrow-\gamma p_{x}} \frac{\{V, H\}\left(x, y, p_{x}, p_{y}\right)}{\left(\gamma(x, y) p_{x}+p_{y}\right) l(x, y)} \\
& =-\frac{1}{l(x, y)} \frac{\partial\{V, H\}}{\partial p_{y}}\left(x, y, p_{x},-\gamma(x, y) p_{x}\right) .
\end{aligned}
$$

Usando (3.46) y (3.55), no es difícil (aunque extenso) ver que

$$
\begin{aligned}
\lambda\left(x, y, p_{x},-\gamma(x, y) p_{x}\right)= & {\left[\frac{1}{2}(B \gamma+C)+\frac{2\left(b_{x}-\gamma c_{x}\right) \delta-b \delta_{x}-c \delta_{y}}{2 l}-\gamma_{x}(a-b \gamma)\right.} \\
& \left.-\gamma_{y}(b-c \gamma)\right] p_{x}^{2}+\gamma h_{x}+h_{y}-\frac{b v_{x}+c v_{y}}{l} .
\end{aligned}
$$

Por simplicidad, estamos omitiendo la dependencia en $(x, y)$ de las funciones del miembro derecho. Finalmente, combinando (3.54), (3.55) y (3.56), tenemos sobre $\mu^{-1}(0)$,

$$
\mathfrak{F}_{*}(X)=\frac{1}{l}\left[\left(b_{x}-\gamma c_{x}\right) \delta-\frac{b \delta_{x}+c \delta_{y}}{2}\right] p_{x}^{2}-\frac{b v_{x}+c v_{y}}{l} .
$$

Por lo tanto, $Z_{1}$ está dado por las ecuaciones

$$
\gamma(x, y) p_{x}+p_{y}=0 \quad \text { y } \quad K(x, y) p_{x}^{2}-L(x, y)=0,
$$

donde

$$
K=\left(b_{x}-\gamma c_{x}\right) \delta-\frac{b \delta_{x}+c \delta_{y}}{2} \quad \text { y } \quad L=b v_{x}+c v_{y}
$$


En consecuencia, $Z_{1}$ puede describirse como la preimagen de $(0,0)$ de la función

$$
\mathfrak{G}\left(x, y, p_{x}, p_{y}\right)=\left(\gamma(x, y) p_{x}+p_{y}, K(x, y) p_{x}^{2}-L(x, y)\right) .
$$

Queremos ver ahora que el push-forward de esta función [omitiendo nuevamente la dependencia en $(x, y)$ ]

$$
\mathfrak{G}_{*}=\left(\begin{array}{cccc}
\gamma_{x} p_{x} & \gamma_{y} p_{x} & \gamma & 1 \\
K_{x} p_{x}^{2}-L_{x} & K_{y} p_{x}^{2}-L_{y} & 2 K p_{x} & 0
\end{array}\right)
$$

tiene rango máximo alrededor de $(\mathbf{0}, \mathbf{0})$, i.e. $\mathfrak{G}$ es una submersión cuando se restringe a algún entorno abierto de $(\mathbf{0}, \mathbf{0})$. En $(\mathbf{0}, \mathbf{0})$, dicho push-forward está dado por

$$
\mathfrak{G}_{*,(\mathbf{0}, \mathbf{0})}=\left(\begin{array}{cccc}
0 & 0 & \gamma(\mathbf{0}) & 1 \\
-L_{x}(\mathbf{0}) & -L_{y}(\mathbf{0}) & 0 & 0
\end{array}\right) .
$$

Notemos que el gradiente de la función $L$ puede escribirse con notación matricial como

$$
\left(\begin{array}{l}
L_{x} \\
L_{y}
\end{array}\right)=\left(\begin{array}{ll}
v_{x x} & v_{x y} \\
v_{x y} & v_{y y}
\end{array}\right)\left(\begin{array}{l}
b \\
c
\end{array}\right)+\left(\begin{array}{ll}
b_{x} & c_{x} \\
b_{y} & c_{y}
\end{array}\right)\left(\begin{array}{l}
v_{x} \\
v_{y}
\end{array}\right),
$$

y dado que $\mathbf{0}$ es un punto crítico de $v$, tenemos que en $\mathbf{0}$,

$$
\left(\begin{array}{l}
L_{x} \\
L_{y}
\end{array}\right)=\left(\begin{array}{ll}
v_{x x} & v_{x y} \\
v_{x y} & v_{y y}
\end{array}\right)\left(\begin{array}{l}
b \\
c
\end{array}\right) .
$$

Pero sabemos que el la matriz Hessiana de $v$ es invertible (puesto que es definida positiva) y que $c$ es siempre positivo. Luego, el gradiente de $L$ no puede anularse en $\mathbf{0}$, o en otras palabras, $\mathbf{0}$ no es un punto crítico de $L$. Esto implica que (3.59) tiene rango máximo en $(\mathbf{0}, \mathbf{0})$. En consecuencia, existe un subconjunto abierto $T_{1} \subset T^{*} U$ que contiene a $(\mathbf{0}, \mathbf{0})$ y tal que $Z_{1} \cap T_{1}$ es una subvariedad de $\mu^{-1}(0) \cap T_{1}$.

2. Consideremos ahora el subconjunto $Z_{2} \subset Z_{1} \cap T_{1}$ definido por $\mathfrak{G}_{*}(X)\left(x, y, p_{x}, p_{y}\right)=0$. Un cálculo sencillo muestra que $Z_{2}$ está dado por los puntos de $Z_{1} \cap T_{1}$ tales que

$$
\left[(a-b \gamma)\left(K_{x} p_{x}^{2}-L_{x}\right)+(b-c \gamma)\left(K_{y} p_{x}^{2}-L_{y}\right)-2 K\left(\frac{1}{2} B p_{x}^{2}+h_{x}\right)\right] p_{x}=0 .
$$

A partir de ahora, supongamos que

$$
K(\mathbf{0}) \neq 0 .
$$

Observemos que, como $\delta(x, 0)=\mathfrak{g}(x)$, tenemos $\delta_{x}(\mathbf{0})=\mathfrak{g}^{\prime}(0)$, y usando la ecuación cinética en el origen, obtenemos

$$
\delta_{y}(\mathbf{0})=\left[\frac{B \mathfrak{g}-(a-b \gamma) \mathfrak{g}^{\prime}(0)}{b-c \gamma}\right](\mathbf{0})
$$

Luego [ver (3.58)]

$$
K(\mathbf{0})=\left[\left(b_{x}-\gamma c_{x}-\frac{B c}{2(b-c \gamma)}\right) \mathfrak{g}(0)+\frac{1}{2}\left(\frac{\Delta}{b-c \gamma}\right) \mathfrak{g}^{\prime}(0)\right](\mathbf{0}),
$$


y consecuentemente, la condición $K(\mathbf{0}) \neq 0$ es precisamente la Ec. (3.50). Bajo esta suposición, podemos reemplazar $p_{x}^{2}$ por $\frac{L}{K}$ en (3.61) [ver (3.57)], y entonces hallamos

$$
\left[(a-b \gamma)\left(K_{x} \frac{L}{K}-L_{x}\right)+(b-c \gamma)\left(K_{y} \frac{L}{K}-L_{y}\right)-2 K\left(\frac{1}{2} B \frac{L}{K}+h_{x}\right)\right] p_{x}=0,
$$

o equivalentemente,

$$
\left[(a-b \gamma)\left(\frac{L}{K}\right)_{x}+(b-c \gamma)\left(\frac{L}{K}\right)_{y}+B \frac{L}{K}+2 h_{x}\right] K p_{x}=0
$$

en algún subconjunto abierto $U^{\prime} \subset U$ que contiene a $\mathbf{0}$ (donde $K$ sea no nulo). Más aun, dado que $L(\mathbf{0})=0$ [ver (3.58)] y $h_{x}(\mathbf{0})=0$, tenemos que en $\mathbf{0}$

$$
\left(\frac{L}{K}\right)_{x}=\frac{L_{x} K-L K_{x}}{K^{2}}=\frac{L_{x}}{K},\left(\frac{L}{K}\right)_{y}=\frac{L_{y} K-L K_{y}}{K^{2}}=\frac{L_{y}}{K},
$$

y así, la expresión entre corchetes en (3.62) toma la siguiente forma en $\mathbf{0 :}$

$$
(a-b \gamma) L_{x}+(b-c \gamma) L_{y}
$$

A su vez, usando (3.60), esta última expresión puede reescribirse como

$$
(a, b)\left[\begin{array}{ll}
v_{x x} & v_{x y} \\
v_{x y} & v_{y y}
\end{array}\right]\left(\begin{array}{l}
b \\
c
\end{array}\right)-\gamma(b, c)\left[\begin{array}{ll}
v_{x x} & v_{x y} \\
v_{x y} & v_{y y}
\end{array}\right]\left(\begin{array}{l}
b \\
c
\end{array}\right) .
$$

Por lo tanto, si suponemos que en $\mathbf{0}$,

$$
\gamma \neq \frac{(a, b)\left[\begin{array}{ll}
v_{x x} & v_{x y} \\
v_{x y} & v_{y y}
\end{array}\right]\left(\begin{array}{l}
b \\
c
\end{array}\right)}{(b, c)\left[\begin{array}{ll}
v_{x x} & v_{x y} \\
v_{x y} & v_{y y}
\end{array}\right]\left(\begin{array}{l}
b \\
c
\end{array}\right)},
$$

se sigue que (3.62) se cumple sólo si $p_{x}=0$ alrededor de $(x, y)=(0,0)$. Vale la pena señalar que esta condición es compatible con (3.41) y con los puntos 1 y 2 del Lema 3.2. Notemos que, usando la Ec. (3.40), la condición de arriba se traduce precisamente en (3.51) y (3.52). En conclusión, existe un entorno abierto $T_{2}^{\prime}:=\pi^{-1}\left(U^{\prime}\right) \subset T^{*} U$ (que contiene a $\mathbf{0}$ ) tal que el subconjunto $Z_{2} \cap T_{2}^{\prime}$ está dado por

$$
\gamma p_{x}+p_{y}=0, \quad K p_{x}^{2}-L=0, \quad p_{x}=0,
$$

o equivalentemente

$$
p_{x}=p_{y}=L=0 .
$$

Esto significa que $Z_{2} \cap T_{2}^{\prime}$ puede ser descripto como la preimagen de $(0,0,0)$ de la función

$$
\mathfrak{H}\left(x, y, p_{x}, p_{y}\right)=\left(p_{y}, p_{x}, L(x, y)\right) .
$$


El push-forward de $\mathfrak{H}$ en $(\mathbf{0}, \mathbf{0})$ está dado por

$$
\mathfrak{H}_{*,(\mathbf{0}, \mathbf{0})}=\left(\begin{array}{cccc}
0 & 0 & 0 & 1 \\
0 & 0 & 1 & 0 \\
L_{x}(\mathbf{0}) & L_{y}(\mathbf{0}) & 0 & 0
\end{array}\right) .
$$

Nuevamente, como $L_{x}(\mathbf{0})$ y $L_{y}(\mathbf{0})$ no pueden anularse simultáneamente, concluimos que la aplicación $\mathfrak{H}_{*,(\mathbf{0}, 0)}$ tiene rango máximo. Entonces, existe dentro de $T_{2}^{\prime}$ un entorno abierto $T_{2}$ de $(\mathbf{0}, \mathbf{0})$ tal que $Z_{2} \cap T_{2}$ es una subvariedad de $Z_{1} \cap T_{1} \cap T_{2}$.

3. Consideremos ahora el subconjunto $Z_{3} \subset Z_{2} \cap T_{2}$ definido por $\mathfrak{H}_{*}(X)\left(x, y, p_{x}, p_{y}\right)=0$. Usando (3.53), (3.55) y (3.56), se sigue que, sobre $Z_{2} \cap T_{2}$ [ver (3.63)]

$$
X=-h_{x} \frac{\partial}{\partial p_{x}}+\gamma h_{x} \frac{\partial}{\partial p_{y}},
$$

de manera que, si $\mathfrak{H}_{*}(X)=0$, es necesario que $h_{x}=0$. Pero en tal caso, usando la ecuación potencial

$$
(a-b \gamma) v_{x}+(b-c \gamma) v_{y}=\delta h_{x}
$$

o equivalentemente, $a v_{x}+b v_{y}-\gamma L=\delta h_{x}$, tenemos que, sobre $\pi\left(Z_{3}\right)$,

$$
L=b v_{x}+c v_{y}=0 \quad \text { y } \quad a v_{x}+b v_{y}=0,
$$

i.e.

$$
\mathbb{H}\left(\begin{array}{c}
v_{x} \\
v_{y}
\end{array}\right)=0 .
$$

Esto es posible si y sólo si todos los puntos de $\pi\left(Z_{3}\right)$ son críticos para $v$. Por el Lema de Morse, puesto que $\mathbf{0}$ es un punto crítico no degenerado de $v$, existe un entorno $U^{\prime \prime} \subset U$ de $\mathbf{0}$ tal que $\pi\left(Z_{3}\right) \cap U^{\prime \prime}=\{\mathbf{0}\}$. Pero $p_{x}=p_{y}=0$ sobre $Z_{3}$, lo cual implica que $Z_{3} \cap T_{3}=\{(\mathbf{0}, \mathbf{0})\}=\{\bar{\alpha}\}$, $\operatorname{con} T_{3}:=\pi^{-1}\left(U^{\prime \prime}\right)$.

Resumiendo, si definimos $T:=T_{1} \cap T_{2} \cap T_{3}$ y $\mathcal{S}_{0}:=\mu^{-1}(0) \cap T$, de (1.29) obtenemos $\mathcal{S}_{1}=Z_{1} \cap T$, que es una subvariedad de $\mathcal{S}_{0}, \mathcal{S}_{2}=Z_{2} \cap T$, que es una subvariedad de $\mathcal{S}_{1}$, y $\mathcal{S}_{3}=\{\bar{\alpha}\}$. Por lo tanto, los tres puntos del lema son inmediatos.

En conclusión, si la Ec. (3.42) se cumple, podemos asegurar que el sistema es localmente asintóticamente estabilizable. Recíprocamente, si podemos asegurar que el sistema es asintóticamente estabilizable mediante la existencia de una función de Lyapunov simple, entonces en particular el sistema es estabilizable, y el Teorema 3.2 implica que (3.42) se cumple. En otras palabras, el Teorema 3.2 también es válido reemplazando el término "estabilizable” por "asintóticamente estabilizable”. Más precisamente: 
Teorema 3.3. Consideremos un sistema subactuado $(H, \mathcal{W})$ sobre una 2-variedad suave $Q$, y consideremos un punto crítico $\bar{\alpha}=0_{\bar{q}} \in T_{\bar{q}}^{*} Q$ de $X_{H}$. Fijemos una carta local adaptada a $W$ y centrada en $\bar{q}$, y consideremos las correspondientes expresiones locales de $H$ dadas por (3.3) y (3.33). Si la Ec. (3.42) se cumple, entonces $(H, \mathcal{W})$ es localmente asintóticamente estabilizable en $\bar{\alpha}$, i.e. existe un campo vectorial $Y$, definido al menos en un entorno de $\bar{\alpha}$, tal que el sistema a lazo cerrado $(H, Y)_{\mathcal{W}}$ es asintóticamente estable en $\bar{\alpha}$. Recíprocamente, si $(H, \mathcal{W})$ es localmente asintóticamente estabilizable en $\bar{\alpha}, y$ dicha estabilidad puede asegurarse mediante la existencia de una función de Lyapunov simple, entonces (3.42) se cumple.

\subsubsection{Ejemplo: péndulo con disco invertido}

Para terminar el capítulo presentamos un ejemplo de un sistema subactuado con dos grados de libertad para ilustrar los resultados de la sección anterior, el péndulo con disco invertido:

- el espacio de configuraciones es $Q=S^{1} \times S^{1}$, cuyas coordenadas quasi-globales naturales denotaremos por $(\theta, \psi)$;

- el Hamiltoniano está dado por

$$
H\left(\theta, \psi, p_{\theta}, p_{\psi}\right)=\frac{1}{2}\left(p_{\theta}, p_{\psi}\right)\left[\begin{array}{ll}
a & b \\
b & c
\end{array}\right]\left(\begin{array}{l}
p_{\theta} \\
p_{\psi}
\end{array}\right)+M(1+\cos \theta),
$$

donde $a, b, c, M$ son constantes y $a, b, M, a c-b^{2}$ son estrictamente positivos;

- y el espacio de actuación está dado por el subfibrado $W=\operatorname{span}\{\mathrm{d} \psi\}$.

Usando el método LCB simple, vamos a encontrar una señal de control $Y$ para el sistema y una función de Lyapunov simple $V$ relacionada que harán al sistema a lazo cerrado $(H, Y)_{\mathcal{W}}$ asintóticamente estable en $\left(\theta, \psi, p_{\theta}, p_{\psi}\right)=(0,0,0,0)=(\mathbf{0}, \mathbf{0})$.

Volvamos por un momento a la Sección 3.2.1 y reemplacemos $x$ por $\theta$ e $y$ por $\psi$. La Ec. (3.42) en este caso dice que (puesto que $h_{\theta \psi}(\mathbf{0})=0$ )

$$
b h_{\theta \theta}(\mathbf{0}) \neq 0 \quad \text { o bien } \quad h_{\theta \theta}(\mathbf{0})>0,
$$

que es equivalente a $h_{\theta \theta}(\mathbf{0}) \neq 0$, pues $b \neq 0$. Es claro que dicha condición se cumple, puesto que $h_{\theta \theta}(\mathbf{0})=-M \neq 0$. Por lo tanto, el péndulo con disco invertido es asintóticamente estabilizable, alrededor de $(\mathbf{0}, \mathbf{0})$, como es bien sabido. Por otra parte, de acuerdo con (3.36), tenemos que $B=0$. Luego, las ecuaciones cinéticas y potenciales para este sistema se escriben [recordar (3.37) y (3.38)]

$$
(a-b \gamma) \delta_{\theta}+(b-c \gamma) \delta_{\psi}=0
$$

y

$$
(a-b \gamma) v_{\theta}+(b-c \gamma) v_{\psi}=-M \delta \sin \theta,
$$


respectivamente. Construyamos ahora una solución $(\delta, \gamma, v)$ de estas ecuaciones, con $\delta>0$ y $v$ definidas positivas alrededor de $\mathbf{0}$. Consideraremos $\gamma$ constante. Siguiendo los pasos de la Sección 3.2.2, es suficiente con tomar $\gamma$ tal que $\gamma \neq b / c$ [ver (3.41)] y, usando la Ec. (3.28) del Lema 3.2 (dado que $\left.h_{\theta \theta}(\mathbf{0})=-M<0\right)$, pedir que

$$
|\gamma|>\frac{a}{b} \quad \text { y } \quad-\gamma b M<0
$$

La segunda desigualdad dice que $\gamma$ es positivo, de manera que estas ecuaciones imponen únicamente la condición $\gamma>a / b$. Notemos también que, como $a, b, c, a c-b^{2}>0$, tenemos que $a / b>b / c$. Luego, la totalidad de las condiciones sobre la constante $\gamma$ se reduce a

$$
\gamma>\frac{a}{b}
$$

Observación 3.8. Vale la pena notar que, para este sistema, no es posible escoger $\gamma=b / c$. De hecho, en tal caso, de acuerdo con los cálculos llevados a cabo en la Sección 3.2.3, la positividad de $\delta$ y $v$ hubiesen implicado que $h_{\theta \theta}(\mathbf{0})>0$, que no es válido para el sistema del ejemplo.

Con respecto a las condiciones de borde que dan origen a las soluciones $\delta \mathrm{y} v$, es decir las funciones $\mathfrak{g}$ y $\mathfrak{f}$ respectivamente, debemos imponer [ver (3.43) y (3.44)]

$$
\mathfrak{g}(0)>0, \quad \mathfrak{f}(0)=\mathfrak{f}^{\prime}(0)=0 \quad \text { y } \quad \mathfrak{f}^{\prime \prime}(0)>\frac{h_{\theta \theta}^{2}(\mathbf{0}) \mathfrak{g}(0)}{(a-b \gamma) h_{\theta \theta}(\mathbf{0})}=-\frac{M \mathfrak{g}(0)}{a-b \gamma} .
$$

Y para asegurar la estabilizabilidad asintótica, de acuerdo con (3.50), (3.51) y (3.52) del Lema 3.3,

$$
\frac{\mathfrak{g}^{\prime}(0)}{\mathfrak{g}(0)} \neq 0, \quad \text { i.e. } \quad \mathfrak{g}^{\prime}(0) \neq 0,
$$

y

$$
\gamma \neq \frac{\xi^{2} a b \mathfrak{f}^{\prime \prime}(0)-\xi\left[M \mathfrak{g}(0)+\zeta \mathfrak{f}^{\prime \prime}(0)\right]\left(a c+b^{2}\right)+\zeta M \mathfrak{g}(0)+\zeta^{2} \mathfrak{f}^{\prime \prime}(0) b c}{\xi^{2} b^{2} \mathfrak{f}^{\prime \prime}(0)-2 \xi\left[M \mathfrak{g}(0)+\zeta \mathfrak{f}^{\prime \prime}(0)\right] b c+\zeta M \mathfrak{g}(0)+\zeta^{2} \mathfrak{f}^{\prime \prime}(0) c^{2}},
$$

donde $\zeta:=a-b \gamma \mathrm{y} \xi:=b-c \gamma$.

Tomemos entonces cualquier constante $\gamma>a / b$, una función $\mathfrak{g}$ tal que $\mathfrak{g}(0)>0$ y $\mathfrak{g}^{\prime}(0) \neq 0$, y una función ${ }^{5} \mathfrak{f}$ tal que $\mathfrak{f}(0)=\mathfrak{f}^{\prime}(0)=0$ y $\mathfrak{f}^{\prime \prime}(0)$ cumpliendo (3.66) y (3.67), y apliquemos el Método de Características a las Ecuaciones (3.64) y (3.65), con condiciones de borde sobre $\psi=0$ dadas por $\mathfrak{g}$ y $\mathfrak{f}$. Las ecuaciones características para (3.64) son

$$
\begin{array}{ll}
\dot{\theta}=a-b \gamma, & \theta(0)=\theta_{0}, \\
\dot{\psi}=b-c \gamma, & \psi(0)=0, \\
\dot{\delta}=0, & \delta(0)=\mathfrak{g}\left(\theta_{0}\right) .
\end{array}
$$

Luego

$$
\theta(t)=(a-b \gamma) t+\theta_{0}, \quad \psi(t)=(b-c \gamma) t,
$$

\footnotetext{
${ }^{5}$ Adicionalmente, las funciones $\mathfrak{g}$ y $\mathfrak{f}$ pueden escogerse con período $2 \pi$ para buscar una solución quasi-global.
} 
y definiendo $\Upsilon:=(a-b \gamma) /(b-c \gamma)$ hallamos

$$
\delta(\theta, \psi)=\mathfrak{g}(\theta-\Upsilon \psi) .
$$

La ecuación característica para (3.65) (y para la solución $\delta$ recién descripta) es

$$
\dot{v}=-M \mathfrak{g}\left(\theta_{0}\right) \sin \left((a-b \gamma) t+\theta_{0}\right),
$$

e integrando obtenemos

$$
v(\theta, \psi)=\frac{M \mathfrak{g}(\theta-\Upsilon \psi)}{a-b \gamma}(\cos \theta-\cos (\theta-\Upsilon \psi))+\mathfrak{f}(\theta-\Upsilon \psi) .
$$

Finalmente, con $\delta$ y $v$ dadas por (3.68) y (3.69), y considerando cualquier función positiva $l$, obtenemos de las Ecs. (3.46),(3.47) y (3.48) la señal de control $Y$ y la función de Lyapunov $V$ que estábamos buscando. 


\section{SOLUCIONES POR CUADRATURAS DE LAS ECUACIONES CINÉTICAS Y POTENCIALES} PARA SISTEMAS 1-SUBACTUADOS

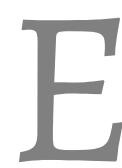

$\mathrm{N}$ el capítulo anterior probamos un resultado de existencia de soluciones para las ecuaciones cinéticas y potenciales del método LCB simple. Tales soluciones pueden utilizarse para construir la señal de control $Y$ que estabilice a un sistema subactuado $(H, \mathcal{W})$ (con $H$ simple) alrededor de un punto de equilibrio $\bar{\alpha}$ del sistema no actuado, así como también una función de Lyapunov simple $V$ para $X_{H}+Y$ y $\bar{\alpha}$. Un resultado análogo había sido desarrollado anteriormente (y de manera independiente) en el contexto del energy shaping para las condiciones de matching cinéticas y potenciales. Sin embargo, en ninguno de los dos casos la demostración es constructiva, sino que se utiliza el Método de Características para asegurar la existencia de soluciones. Si bien en la literatura tal construcción se lleva a cabo para ejemplos concretos, no existe a nuestro entender un procedimiento sistemático que permita construir una solución explícita de las ecuaciones cinéticas y potenciales (o equivalentemente de las condiciones de matching del mismo nombre).

Este punto no es menor, puesto que el objetivo último de un método de estabilización es construir la señal de control que estabilice al sistema. Al probar resultados sobre la existencia de soluciones de las ecuaciones cinéticas y potenciales, sabemos que dicha señal existe pero no tenemos una guía que nos indique cómo contruirla.

En este capítulo, presentaremos dos procedimientos para encontrar explícitamente soluciones locales. En primer lugar, en la Sección \$4.1, encontraremos nuevas expresiones para las ecuaciones cinéticas y potenciales. El desarrollo de esta sección es, en cierto sentido, una generalización del cambio de variables dependientes a las variables $(\delta, \gamma, l)$ en el término cinético que utilizamos para encontrar las ecuaciones (3.12) y (3.15) en el capítulo anterior.

Seguidamente, en la Sección $\$ 4.2$, presentamos el primer procedimiento para construir soluciones 
4. Soluciones por cuadraturas de las ecuaciones cinéticas y potenciales para sistemas 1-subactuados

explícitas de las ecuaciones potenciales. Este primer resultado da una prescripción para construir dichas soluciones suponiendo que contamos con una solución de las ecuaciones cinéticas que además cumplen con una condición de integrabilidad. Esta idea es también la que se utliza en [30] para mostrar condiciones de integrabilidad que aseguran la existencia de soluciones locales de las condiciones de matching potenciales del energy shaping a partir de una solución de las condiciones de matching cinéticas. ${ }^{1}$ En dicho artículo, el autor utiliza la teoría de integrabilidad de Goldschmidt [17], suponiendo que el subfibrado de actuación $W$ es integrable, para probar un resultado de existencia (no constructivo) en la categoría analítica. Nuestro procedimiento, por el contrario, es válido en la categoría $C^{\infty}$ y no supone la integrabilidad de $W$. Más aun, la solución puede computarse por cuadraturas y, adicionalmente, mostramos una condición necesaria y suficiente para que la solución construida sea definida positiva alrededor de $\bar{q}$.

Por último, en la Sección $\$ 4.3$, aplicamos el procedimiento desarrollado en $\$ 4.2$ para hallar una solución de las ecuaciones cinéticas para sistemas 1-subactuados que automáticamente cumplen la condición de integrabilidad arriba mencionada. Al igual que antes, esta solución también es computable por cuadraturas. Además, mostramos que la condición de positividad de las soluciones para los sistemas 1-subactuados se reduce a que el sistema sea de tipo 2 (ver Definición 3.2). Esto nos permite dar un conjunto completo de instrucciones para construir soluciones locales de las ecuaciones cinéticas y potenciales.

\subsection{Nueva expresión de las ecuaciones cinéticas y potenciales a partir de un cambio de variables dependientes}

Sea $V$ un subfibrado lineal de $T^{*} Q$ de rango $s=n-m$ y supongamos que $T^{*} Q=V \oplus W$. Asociadas a esta descomposición, tenemos las proyecciones canónicas $p_{V}: T^{*} Q \rightarrow V$ y $p_{W}: T^{*} Q \rightarrow W$, tales que para todo $\alpha=\alpha_{V}+\alpha_{W}$ tal que $\alpha_{V} \in V$ y $\alpha_{W} \in W$

$$
p_{V}(\alpha)=\alpha_{V} \quad \text { y } \quad p_{W}(\alpha)=\alpha_{W} .
$$

Tenemos también las inclusiones $i_{V}: V \rightarrow T^{*} Q$ e $i_{W}: W \rightarrow T^{*} Q$. En estas condiciones es claro que el fibrado tangente $T Q$ es isomorfo a $V^{*} \oplus W^{*}$ via el isomorfismo

$$
\chi(v)=\left(i_{V}^{*}(v), i_{W}^{*}(v)\right) \in V^{*} \oplus W^{*} \quad \forall v \in T Q
$$

con inversa

$$
\chi^{-1}(u, w)=p_{V}^{*}(u)+p_{W}^{*}(w) \in T Q \quad \forall(u, w) \in V^{*} \oplus W^{*} .
$$

Asociada a la descomposición $V \oplus W$ tenemos la siguiente definición

\footnotetext{
${ }^{1}$ Vale la pena decir que en [30], se trabaja con el energy shaping en su formulación Lagrangiana, a diferencia de esta tesis, en la cual trabajamos con la formulación Hamiltoniana
} 
Definición 4.1. Sea $\phi \in \Gamma\left(S_{+}^{2}(T Q)\right)$ una forma bilineal simétrica y definida positiva (ver Sección \$1.1). Definimos entonces $\delta_{\phi} \in \Gamma\left(S_{+}^{2}\left(V^{*}\right)\right), l_{\phi} \in \Gamma\left(S_{+}^{2}\left(W^{*}\right)\right)$ y un morfismo de fibrados vectoriales $\gamma_{\phi}$ : $V \rightarrow W$ mediante las fórmulas

$$
\begin{aligned}
l_{\phi}\left(\sigma_{1}, \sigma_{2}\right) & =\phi\left(i_{W}\left(\sigma_{1}\right), i_{W}\left(\sigma_{2}\right)=\phi\left(\sigma_{1}, \sigma_{2}\right), \quad \forall \sigma_{1}, \sigma_{1} \in W,\right. \\
\gamma_{\phi} & =l_{\phi}^{b} \circ i_{W}^{*} \circ \phi^{\sharp} \circ i_{V}, \\
\delta_{\phi}\left(\lambda_{1}, \lambda_{2}\right) & =\phi\left(i_{V}\left(\lambda_{1}\right), i_{V}\left(\lambda_{2}\right)\right)-l_{\phi}\left(\gamma_{\phi}\left(\lambda_{1}\right), \gamma_{\phi}\left(\lambda_{2}\right)\right), \quad \forall \lambda_{1}, \lambda_{2} \in V .
\end{aligned}
$$

De ahora en más, usaremos índices griegos $\mu, \nu, \sigma, \tau=1, \ldots, s$, índices latinos $a, b, c, d=1, \ldots m$ e índices latinos $i, j, k, l=1, \ldots, n$.

Supongamos que $\left\{E^{1}, \ldots, E^{s}\right\}$ es un marco en $V$ y que $\left\{E^{s+1}, \ldots, E^{n}\right\}$ es un marco de $W$. Se sigue entonces que $\left\{E^{1}, \ldots, E^{n}\right\}$ es un marco en $T^{*} Q$. A su vez, tenemos los marcos duales $\left\{E_{1}, \ldots, E_{s}\right\}$ en $V^{*}$ y $\left\{E_{s+1}, \ldots, E_{n}\right\}$ en $W^{*}$. Asociados a estos marcos y al isomorfismo $\chi$, tenemos un marco en $T Q$ dado por $\left\{\chi^{-1}\left(E_{1}\right), \ldots, \chi^{-1}\left(E_{n}\right)\right\}$. Notemos que

$$
\begin{array}{ll}
\left\langle E^{\mu}, \chi^{-1}\left(E_{s+a}\right)\right\rangle=0, & \left\langle E^{s+a}, \chi^{-1}\left(E_{\nu}\right)\right\rangle=0, \\
\left\langle E^{\mu}, \chi^{-1}\left(E_{\nu}\right)\right\rangle=\delta_{\nu}^{\mu}, & \left\langle E^{s+b}, \chi^{-1}\left(E_{s+a}\right)\right\rangle=\delta_{a}^{b} .
\end{array}
$$

Luego, el marco $\left\{\chi^{-1}\left(E_{i}\right)\right\}_{i=1}^{n}$ es el marco dual a $\left\{E^{j}\right\}_{j=1}^{n}$ y por lo tanto lo denotaremos también $E_{i}$. Diremos que el conjunto de marcos definidos arriba para los fibrados involucrados son marcos adaptados a la descomposición de $T^{*} Q$.

Veamos cómo se escriben las formas bilineales $l_{\phi}$ y $\delta_{\phi}$ junto con el morfismo $\gamma_{\phi}$ usando los marcos adaptados introducidos en el párrafo anterior. En primer lugar, notemos que

$$
\begin{gathered}
p_{V}\left(E^{\mu}\right)=E^{\mu}, \quad p_{V}\left(E^{s+a}\right)=0, \quad p_{W}\left(E^{\mu}\right)=0, \quad p_{W}\left(E^{s+a}\right)=E^{s+a}, \\
i_{V}\left(E^{\mu}\right)=E^{\mu}, \quad i_{W}\left(E^{s+a}\right)=E^{s+a}, \\
i_{V}^{*}\left(E_{s+a}\right)=0, \quad i_{W}^{*}\left(E_{\mu}\right)=0, \\
i_{V}^{*}\left(E_{\mu}\right)=i_{V}^{*} \circ p_{V}^{*}\left(E_{\mu}\right)=\left(p_{V} \circ i_{V}\right)^{*}\left(E_{\mu}\right)=\left(i d_{V}\right)^{*}\left(E_{\mu}\right)=E_{\mu}, \\
i_{W}^{*}\left(E_{s+a}\right)=i_{W}^{*} \circ p_{W}^{*}\left(E_{s+a}\right)=\left(p_{W} \circ i_{W}\right)^{*}\left(E_{s+a}\right)=\left(i d_{W}\right)^{*}\left(E_{s+a}\right)=E_{s+a} .
\end{gathered}
$$

Luego, definiendo $\check{\mathbb{V}}^{i j}:=\phi\left(E^{i}, E^{j}\right)=\check{\mathbb{V}}^{j i}$, tenemos que

$$
l_{\phi}^{a b}:=l_{\phi}\left(E^{s+a}, E^{s+b}\right)=\check{\mathbb{V}}^{s+a, s+b} .
$$

Es claro entonces que

$$
\phi^{\sharp}\left(E^{i}\right)=\check{\mathbb{V}}^{i j} E_{j},
$$

\footnotetext{
${ }^{2}$ Para evitar confusiones, usaremos la notación $\breve{V}$ y $\check{\mathbb{H}}$ para denotar las entradas de la representación matricial de $\phi$ y $\rho$ en marcos distintos a los coordenados.
} 
4. Soluciones por cuadraturas de las ecuaciones cinéticas y potenciales para sistemas 1-subactuados

y que

$$
\phi^{b}\left(E_{i}\right)=\check{\mathbb{V}}_{i j} E^{j},
$$

con $\check{\mathbb{V}}_{i k} \check{\mathbb{V}}^{k j}=\delta_{i}^{j}$. Por otra parte, definamos [recordar (4.1)]

$$
\left(\gamma_{\phi}\right)_{a}^{\mu}:=\left\langle E_{s+a}, \gamma_{\phi}\left(E^{\mu}\right)\right\rangle=\left\langle E_{s+a},\left(l_{\phi}^{b} \circ i_{W}^{*} \circ \phi^{\sharp} \circ i_{V}\right)\left(E^{\mu}\right)\right\rangle=\left(l_{\phi}\right)_{a b} \check{\mathbb{V}}^{s+b, \mu},
$$

y usando (4.2), definamos

$$
\delta^{\mu \nu}:=\delta_{\phi}\left(E^{\mu}, E^{\nu}\right)=\check{\mathbb{V}}^{\mu \nu}-\check{\mathbb{V}}^{\mu, s+a}\left(l_{\phi}\right)_{a b} \check{\mathbb{V}}^{s+b, \nu},
$$

Observación 4.1.

1. Siguiendo la notación del Apéndice,

$$
l_{\phi}^{a b}=\check{\mathbb{V}}_{\underline{m}}^{a b}, \quad\left(l_{\phi}\right)_{a b}=\left(\check{\mathbb{V}}_{\underline{m}}\right)_{a b},
$$

de modo que (4.3) se escribe también

$$
\delta^{\mu \nu}:=\delta_{\phi}\left(E^{\mu}, E^{\nu}\right)=\check{\mathbb{V}}^{\mu \nu}-\check{\mathbb{V}}^{\mu, s+a}\left(\check{\mathbb{V}}_{\underline{m}}\right)_{a b} \check{\mathbb{V}}^{s+b, \nu}
$$

2. No deben confundirse los símbolos $\left(l_{\phi}\right)_{a b}$ con los símbolos $\check{\mathbb{V}}_{s+a, s+b}$. El primero representa el elemento $a b$ de la inversa de la matriz $\breve{\mathbb{V}}_{\underline{m}}$, mientras que el segundo es el elemento $s+a, s+b$ de la inversa de $\check{\mathbb{V}}$.

3. En el caso en que los fibrados $V$ y $W$ sean integrables, pueden considerarse los marcos adaptados asociados al (co)marco coordenado. En este caso, los elementos $\check{\mathbb{V}}^{i j}=\mathbb{V}^{i j}$ son los coeficientes de la matriz del isomorfismo $\phi^{\sharp}$ en las coordenadas inducidas en el cotangente por las coordenadas en la base.

Utilizando (4.3) [o (4.4)] y el Corolario A.1, el lema siguiente es de demostración inmediata

Lema 4.1. Si $\phi \in \Gamma\left(S_{+}^{2}(T Q)\right)$ es una forma bilineal simétrica y definida positiva, entonces $\delta_{\phi} \in \Gamma\left(S_{+}^{2}\left(V^{*}\right)\right)$ $y l_{\phi} \in \Gamma\left(S_{+}^{2}\left(W^{*}\right)\right)$ también lo son.

Sea $L(V, W)$ el conjunto de morfismos de fibrados vectoriales entre $V$ y $W$ y sea $\gamma \in L(V, W)$. Denotemos $\gamma_{a}^{\mu}$ a las entradas de la representación matricial de $\gamma$ respecto de los marcos adaptados. Asociado a este morfismo podemos definir dos morfismos de fibrados vectoriales $\gamma_{-} \in L\left(V, T^{*} Q\right)$ y $\gamma_{+} \in L\left(T^{*} Q, W\right)$, dados por

$$
\gamma_{-}(\lambda)=\lambda-\gamma(\lambda), \quad \gamma_{+}(\alpha)=p_{W}(\alpha)+\gamma\left(p_{V}(\alpha)\right)
$$


Observación 4.2. Notemos que

$$
\gamma_{+} \circ \gamma_{-}(\lambda)=\gamma_{+}(\lambda-\gamma(\lambda))=-\gamma(\lambda)+\gamma(\lambda)=0
$$

Dado que $T^{*} Q=V \oplus W$, esto dice que la secuencia

$$
0 \rightarrow V \stackrel{\gamma_{-}}{\longrightarrow} T^{*} Q \stackrel{\gamma_{+}}{\longrightarrow} W \rightarrow 0
$$

es una secuencia exacta corta de fibrados vectoriales, i.e. $\operatorname{ker}\left(\gamma_{+}\right)=\operatorname{Im}\left(\gamma_{-}\right)$.

Definición 4.2. Sea $\gamma \in L(V, W)$ un morfismo de fibrados vectoriales y sean $l \in \Gamma\left(S_{+}^{2}\left(W^{*}\right)\right)$ y $\delta \in$ $\Gamma\left(S_{+}^{2}\left(V^{*}\right)\right)$ formas bilineales simétricas definidas positivas. Definimos entonces una forma bilineal simétrica $\phi_{\delta, \gamma, l} \in \Gamma\left(S^{2}(T Q)\right)$ de la siguiente manera [recordar (4.5)]:

$$
\phi_{\delta, \gamma, l}(\alpha, \beta)=\delta\left(p_{V}(\alpha), p_{V}(\beta)\right)+l\left(\gamma_{+}(\alpha), \gamma_{+}(\beta)\right)
$$

Observación 4.3. Utilizando los marcos adaptados, es fácil ver que

$$
\alpha_{i} \check{\bigvee}^{i j} \beta_{j}=\alpha_{\mu} \delta^{\mu \nu} \beta_{\nu}+\left(\alpha_{s+a}+\gamma_{a}^{\mu} \alpha_{\mu}\right) l^{a b}\left(\beta_{s+b}+\gamma_{b}^{\nu} \beta_{\nu}\right)
$$

donde $\check{\mathbb{V}}^{i j}=\phi_{\delta, \gamma, l}\left(E^{i}, E^{j}\right), \alpha=\alpha_{i} E^{i}$ y $\beta=\beta_{j} E^{j}$. De esta expresión, es inmediato que $\phi_{\delta, \gamma, l} \in$ $\Gamma\left(S_{+}^{2}(T Q)\right)$.

La siguiente proposición nos servirá para efectuar un cambio de variables dependientes, similar al construido en el capítulo anterior para el caso de sistemas regularmente actuados, pero que esta vez servirá para cualquier sistema subactuado. Al igual que en el caso regular, este cambio simplificará considerablemente el aspecto de las ecuaciones cinéticas y potenciales.

Proposición 4.1. La asignación $\Gamma\left(S_{+}^{2}\left(V^{*}\right)\right) \times L(V, W) \times \Gamma\left(S_{+}^{2}\left(W^{*}\right)\right) \rightarrow \Gamma\left(S_{+}^{2}(T Q)\right)$ tal que (ver Definición 4.2)

$$
(\delta, \gamma, l) \mapsto \phi_{\delta, \gamma, l}
$$

es una biyección con inversa (ver Definición 4.1)

$$
\phi \mapsto\left(\delta_{\phi}, \gamma_{\phi}, l_{\phi}\right)
$$

Demostración. Dada $\phi \in \Gamma\left(S_{+}^{2}(T Q)\right)$, computemos $\phi_{\delta_{\phi}, \gamma_{\phi}, l_{\phi}}$. Dados $\alpha, \beta \in T^{*} Q$,

$$
\begin{aligned}
\phi_{\delta_{\phi}, \gamma_{\phi}, l_{\phi}}(\alpha, \beta) & =\delta_{\phi}\left(p_{V}(\alpha), p_{V}(\beta)\right)+l_{\phi}\left(\gamma_{\phi+}(\alpha), \gamma_{\phi+}(\beta)\right) \\
& =\phi\left(p_{V}(\alpha), p_{V}(\beta)\right)-l_{\phi}\left(\gamma_{\phi}\left(p_{V}(\alpha)\right), \gamma_{\phi}\left(p_{V}(\beta)\right)\right)+l_{\phi}\left(\gamma_{\phi+}(\alpha), \gamma_{\phi+}(\beta)\right) \\
& =\phi\left(p_{V}(\alpha), p_{V}(\beta)\right)+l_{\phi}\left(\gamma_{\phi}\left(p_{V}(\alpha)\right), p_{W}(\beta)\right)+l_{\phi}\left(p_{W}(\alpha), \gamma_{\phi}\left(p_{V}(\beta)\right)\right) \\
& +l_{\phi}\left(p_{W}(\alpha), p_{W}(\beta)\right) .
\end{aligned}
$$


Pero

$$
\begin{aligned}
l_{\phi}\left(\gamma_{\phi}\left(p_{V}(\alpha)\right), p_{W}(\beta)\right) & =\left\langle l_{\phi}^{\sharp}\left(\gamma_{\phi}\left(p_{V}(\alpha)\right)\right), p_{W}(\beta)\right\rangle=\left\langle\left(l_{\phi}^{\sharp} \circ l_{\phi}^{b} \circ i_{W}^{*} \circ \phi^{\sharp} \circ i_{V}\right)\left(p_{V}(\alpha)\right), p_{W}(\beta)\right\rangle \\
& =\left\langle\phi^{\sharp}\left(p_{V}(\alpha)\right), p_{W}(\beta)\right\rangle=\phi\left(p_{V}(\alpha), p_{W}(\beta)\right)
\end{aligned}
$$

y de la misma forma

$$
l_{\phi}\left(p_{W}(\alpha), \gamma_{\phi}\left(p_{V}(\beta)\right)\right)=\phi\left(p_{W}(\alpha), p_{V}(\beta)\right)
$$

por lo que

$$
\begin{aligned}
\phi_{\delta_{\phi}, \gamma_{\phi}, l_{\phi}}(\alpha, \beta) & =\phi\left(p_{V}(\alpha), p_{V}(\beta)\right)+\phi\left(p_{V}(\alpha), p_{W}(\beta)\right)+\phi\left(p_{W}(\alpha), p_{V}(\beta)\right)+l_{\phi}\left(p_{W}(\alpha), p_{W}(\beta)\right) \\
& =\phi\left(p_{V}(\alpha), p_{V}(\beta)\right)+\phi\left(p_{V}(\alpha), p_{W}(\beta)\right)+\phi\left(p_{W}(\alpha), p_{V}(\beta)\right)+\phi\left(p_{W}(\alpha), p_{W}(\beta)\right) \\
& =\phi\left(p_{V}(\alpha)+p_{W}(\alpha), p_{V}(\beta)+p_{W}(\beta)\right) \\
& =\phi(\alpha, \beta)
\end{aligned}
$$

para todo $\alpha, \beta \in T^{*} Q$, de modo que $\phi_{\delta_{\phi}, \gamma_{\phi}, l_{\phi}}=\phi$.

Por otro lado, dados $\delta, \gamma$ y $l$,

$$
l_{\phi_{\delta, \gamma, l}}\left(\sigma_{1}, \sigma_{2}\right)=\phi_{\delta, \gamma, l}\left(\sigma_{1}, \sigma_{2}\right)=l\left(\sigma_{1}, \sigma_{2}\right),
$$

para todo $\sigma_{1}, \sigma_{2} \in W$. Además

$$
\begin{aligned}
\left\langle\gamma_{\phi_{\delta, \gamma, l}}(\lambda), w\right\rangle & =\left\langle\left(l_{\phi_{\delta, \gamma, l}^{b}}^{b} \circ i_{W}^{*} \circ \phi_{\delta, \gamma, l}^{\sharp} \circ i_{V}\right)(\lambda), w\right\rangle=\left\langle\left(l^{b} \circ i_{W}^{*} \circ \phi_{\delta, \gamma, l}^{\sharp} \circ i_{V}\right)(\lambda), w\right\rangle \\
& =\phi_{\delta, \gamma, l}\left(\lambda, l^{b}(w)\right)=l\left(\gamma_{+}(\lambda), \gamma_{+}\left(l^{b}(w)\right)\right)=l\left(\gamma(\lambda), l^{b}(w)\right)=\langle\gamma(\lambda), w\rangle,
\end{aligned}
$$

para todo $\lambda \in V$ y $w \in W^{*}$. Finalmente

$$
\begin{aligned}
\delta_{\phi_{\delta, \gamma, l}}\left(\lambda_{1}, \lambda_{2}\right) & =\phi_{\delta, \gamma, l}\left(i_{V}\left(\lambda_{1}\right), i_{V}\left(\lambda_{2}\right)\right)-l_{\phi_{\delta, \gamma, l}}\left(\gamma_{\phi_{\delta, \gamma, l}}\left(\lambda_{1}\right), \gamma_{\phi_{\delta, \gamma, l}}\left(\lambda_{2}\right)\right) \\
& =\phi_{\delta, \gamma, l}\left(i_{V}\left(\lambda_{1}\right), i_{V}\left(\lambda_{2}\right)\right)-l\left(\gamma\left(\lambda_{1}\right), \gamma\left(\lambda_{2}\right)\right) \\
& =\delta\left(\lambda_{1}, \lambda_{2}\right)+l\left(\gamma_{+}\left(\lambda_{1}\right), \gamma_{+}\left(\lambda_{2}\right)\right)-l\left(\gamma\left(\lambda_{1}\right), \gamma\left(\lambda_{2}\right)\right) \\
& =\delta\left(\lambda_{1}, \lambda_{2}\right)+l\left(\gamma\left(\lambda_{1}\right), \gamma\left(\lambda_{2}\right)\right)-l\left(\gamma\left(\lambda_{1}\right), \gamma\left(\lambda_{2}\right)\right) \\
& =\delta\left(\lambda_{1}, \lambda_{2}\right),
\end{aligned}
$$

para todo $\lambda_{1}, \lambda_{2} \in V$.

Por último, la positividad de $\delta_{\phi}$ se establece en el Lema 4.1, la de $\phi_{\delta, \gamma, l}$ en la Observación 4.1 y la de $l_{\phi}$ es inmediata.

Observación 4.4. La construcción anterior asegura que es posible obtener cualquier forma bilineal definida positiva $\phi$ a partir de formas bilineales simétricas y definidas positivas $\delta$ y $l$ y un morfismo de fibrados $\gamma$. Esto permite pensar a la incógnita $\phi$ de las ecuaciones cinéticas y potenciales en términos de tres nuevas incógnitas $\delta, \gamma$ y $l$. En la sección siguiente encontraremos nuevas expresiones de estas ecuaciones en términos de estas nuevas variables. 


\subsubsection{Ecuaciones cinéticas y potenciales en las variables $(\delta, \gamma, l)$}

Siguiendo (2.47) y (2.48), para encontrar expresiones de las ecuaciones cinéticas y potenciales en términos de las variables $\delta, \gamma$ y $l$, es preciso describir el fibrado $\hat{W}=\mathbb{F}^{-1}\left(W^{\circ}\right)=W^{\perp_{\phi}}$ en términos de dichas variables y del complemento $V$. Dicha descripción se establece en el siguiente Lema:

Lema 4.2. Dado $\phi=\phi_{\delta, \gamma, l}$ se tiene que

$$
\hat{W}=\gamma_{-}(V) .
$$

Demostración. Notemos que $\alpha \in \hat{W}$ si y sólo si para todo $\sigma \in W$ se tiene que

$$
\phi_{\delta, \gamma, l}(\alpha, \sigma)=0 \Leftrightarrow \delta\left(p_{V}(\alpha), p_{V}(\sigma)\right)+l\left(\gamma_{+}(\alpha), \gamma_{+}(\sigma)\right)=0 \Leftrightarrow l\left(\gamma_{+}(\alpha), \sigma\right)=0,
$$

y como $l$ es no degenerada esto ocurre si y sólo si (ver Observación 4.2)

$$
\gamma_{+}(\alpha)=0 \Leftrightarrow \alpha \in \operatorname{ker} \gamma_{+}=\gamma_{-}(V) \text {. }
$$

Procedamos ahora a reescribir las ecuaciones. En primer lugar, denotemos $\mathfrak{d}(\lambda)=1 / 2 \delta(\lambda, \lambda) \mathrm{y}$ $\mathfrak{l}(\sigma)=1 / 2 l(\sigma, \sigma)$, las formas cuadráticas asociadas con las formas bilineales $\delta$ y $l$. Con esta notación, si $\mathfrak{v}$ es la forma cuadrática asociada a una forma bilineal $\phi=\phi_{\delta, \gamma, l}$, entonces

$$
\mathfrak{v}=\mathfrak{d} \circ p_{V}+\mathfrak{l} \circ \gamma_{+} .
$$

Proposición 4.2. La función $v$ satisface las ecuaciones potenciales (2.48) para una forma cuadrática $\mathfrak{v}$ asociada a una forma bilineal definida positiva $\phi=\phi_{\delta, \gamma, l}$ si y sólo si $v$ satisface

$$
\left\langle\mathrm{d} v(q), \mathbb{F} \mathfrak{h}\left(\gamma_{-}(\lambda)\right)\right\rangle=\left\langle p_{V}(\mathrm{~d} h(q)), \mathbb{F} \mathfrak{d}(\lambda)\right\rangle, \quad \forall \lambda \in V_{q}, \forall q \in Q .
$$

Demostración. Tomando $\alpha, \beta \in T_{q}^{*} Q$, se sigue de (4.7) que

$$
\begin{aligned}
\langle\beta, \mathbb{F} \mathfrak{v}(\alpha)\rangle & =\left.\frac{\mathrm{d}}{\mathrm{d} s}\right|_{s=0} \mathfrak{v}(\alpha+s \beta)=\left.\frac{\mathrm{d}}{\mathrm{d} s}\right|_{s=0} \frac{1}{2} \delta\left(p_{V}(\alpha+s \beta), p_{V}(\alpha+s \beta)\right) \\
& +\left.\frac{\mathrm{d}}{\mathrm{d} s}\right|_{s=0} \frac{1}{2} l\left(\gamma_{+}(\alpha+s \beta), \gamma_{+}(\alpha+s \beta)\right) \\
& =\left.\frac{\mathrm{d}}{\mathrm{d} s}\right|_{s=0} \mathfrak{d} \circ p_{V}(\alpha+s \beta)+\left.\frac{\mathrm{d}}{\mathrm{d} s}\right|_{s=0} \mathfrak{l} \circ \gamma_{+}(\alpha+s \beta) .
\end{aligned}
$$

En particular, si $\alpha=\gamma_{-}(\lambda) \operatorname{con} \lambda \in V$

$$
\begin{aligned}
\left\langle\beta, \mathbb{F} \mathfrak{v}\left(\gamma_{-}(\lambda)\right)\right\rangle & =\left.\frac{\mathrm{d}}{\mathrm{d} s}\right|_{s=0} \mathfrak{d}\left(\lambda+s p_{V}(\beta)\right) \\
& +\left.\frac{\mathrm{d}}{\mathrm{d} s}\right|_{s=0} \mathfrak{l}\left(-\gamma(\lambda)+\gamma(\lambda)+s \gamma_{+}(\beta)\right) \\
& =\left\langle p_{V}(\beta), \mathbb{F} \mathfrak{d}(\lambda)\right\rangle+\left.\frac{\mathrm{d}}{\mathrm{d} s}\right|_{s=0} s^{2} \mathfrak{l}\left(\gamma_{+}(\beta)\right) \\
& =\left\langle p_{V}(\beta), \mathbb{F} \mathfrak{d}(\lambda)\right\rangle .
\end{aligned}
$$


4. Soluciones por cuadraturas de las ecuaciones cinéticas y potenciales para sistemas 1-subactuados

Evaluando la ecuación (4.9) para $\beta=\mathrm{d} h(q)$,

$$
\left\langle\mathrm{d} h(q), \mathbb{F} \mathfrak{v}\left(\gamma_{-}(\lambda)\right)\right\rangle=\left\langle p_{V}(\mathrm{~d} h(q)), \mathbb{F} \mathfrak{d}(\lambda)\right\rangle, \quad \lambda \in V
$$

Observación 4.5. Vale la pena recordar que $\mathbb{F} \mathfrak{d}: V \rightarrow V^{*}$ es un isomorfismo de fibrados vectoriales. De hecho, usando los marcos adaptados,

$$
\left\langle\mathbb{F} \mathfrak{d}\left(E^{\mu}\right), E^{\nu}\right\rangle=\left.\frac{\mathrm{d}}{\mathrm{d} s}\right|_{s=0} \mathfrak{d}\left(E^{\mu}+s E^{\nu}\right)=\delta^{\mu \nu}=\left\langle\delta^{\sharp}\left(E^{\mu}\right), E^{\nu}\right\rangle,
$$

de donde concluimos que $\mathbb{F} \mathfrak{d}=\delta^{\sharp}$.

Luego, teniendo en cuenta (4.6) y (4.10); $v$ satisface (2.48) si y sólo si cumple (4.8).

Con respecto a las ecuaciones cinéticas, recordemos que si $\nabla$ es una conexión afin (sin torsión) sobre $T^{*} Q$ y $f, g \in C^{\infty}\left(T^{*} Q\right)$, entonces $\mathbb{B}(f+g)=\mathbb{B} f+\mathbb{B} g$. De este modo, siguiendo (4.7), $\mathbb{B} \mathfrak{v}=$ $\mathbb{B}\left(\mathfrak{d} \circ p_{V}\right)+\mathbb{B}\left(\mathfrak{l} \circ \gamma_{+}\right)$. El siguiente lema será de utilidad en la próxima proposición.

Lema 4.3. Sea $\gamma \in L(V, W)$ y sean l una forma bilineal simétrica de $W$ y l su forma cuadrática asociada. Entonces [recordar (4.5)]

$$
\mathbb{B}\left(\mathfrak{l} \circ \gamma_{+}\right)(\alpha)=0, \quad \forall \alpha \in \gamma_{-}(V)
$$

Demostración. Tomemos $X \in T_{\pi(\alpha)} Q$ y consideremos una curva $c:(-\varepsilon, \varepsilon) \rightarrow T^{*} Q$ tal que

$$
q(s):=\pi(c(s)), \quad c(0)=\alpha=\alpha_{i} E^{i}, \quad q^{\prime}(0)=X=X^{i} E_{i}, \quad \frac{D c}{D s}=0,
$$

para un sistema de marcos adaptados. Luego,

$$
\begin{aligned}
\left\langle\mathbb{B}\left(\mathfrak{l} \circ \gamma_{+}\right)(\alpha), X\right\rangle & =\left.\frac{\mathrm{d}}{\mathrm{d} s}\right|_{s=0} \mathfrak{l}\left(\gamma_{+}(c(s))\right)=\left.\frac{1}{2} \frac{\mathrm{d}}{\mathrm{d} s}\right|_{s=0} \gamma_{+}(c(s))_{a} l^{a b}(q(s)) \gamma_{+}(c(s))_{b} \\
& =\left.\frac{\mathrm{d}}{\mathrm{d} s}\right|_{s=0}\left[\gamma_{+}(c(s))_{a}\right] l^{a b}(q(0)) \gamma_{+}(c(0))_{b} \\
& +\left.\frac{1}{2} \gamma_{+}(c(0))_{a} \frac{\mathrm{d}}{\mathrm{d} s}\right|_{s=0}\left[l^{a b}(q(s))\right] \gamma_{+}(c(0))_{b} \\
& =\left.\frac{\mathrm{d}}{\mathrm{d} s}\right|_{s=0}\left[\gamma_{+}(c(s))_{a}\right] l^{a b}(q) \gamma_{+}(\alpha)_{b} \\
& +\left.\frac{1}{2} \gamma_{+}(\alpha)_{a} \frac{\mathrm{d}}{\mathrm{d} s}\right|_{s=0}\left[l^{a b}(q(s))\right] \gamma_{+}(\alpha)_{b} .
\end{aligned}
$$

Luego, si $\alpha \in \gamma_{-}(V)$, es claro que $\gamma_{+}(\alpha)=0$, puesto que $\gamma_{+} \circ \gamma_{-}=0$ (ver Observación 4.2).

Proposición 4.3. La forma cuadrática $\mathfrak{v}$ satisface las ecuaciones cinéticas (2.47) si y sólo si d satisface la ecuación

$$
\left\langle\mathbb{B}\left(\mathfrak{d} \circ p_{V}\right)\left(\gamma_{-}(\lambda)\right), \mathbb{F} \mathfrak{h}\left(\gamma_{-}(\lambda)\right)\right\rangle=\left\langle p_{V}\left(\mathbb{B h}\left(\gamma_{-}(\lambda)\right)\right), \mathbb{F} \mathfrak{d}(\lambda)\right\rangle, \quad \forall \lambda \in V
$$


Demostración. La demostración es inmediata utilizando (4.9) para $\beta=\mathbb{B h}\left(\gamma_{-}(\lambda)\right)$, el lema anterior y la Ecuación (2.47).

Observación 4.6. Es importante observar que la forma bilineal $l$ no aparece en las ecuaciones (4.8) y (4.11). Esto dice que las expresiones de las ecuaciones cinéticas y potenciales en términos de las variables $\delta, \gamma$ y $l$ se desacoplan de manera tal que $l$ puede escogerse libremente mientras que $\delta$ y $\gamma$ deben satisfacer (4.8) y (4.11).

\subsubsection{Expresiones en coordenadas}

En esta sección buscaremos expresiones en coordenadas para las ecuaciones (4.8) y (4.11). Tomemos entonces una carta coordenada $(U, \varphi)$. Asociado a estas coordenadas tenemos los marcos coordenados $\left\{\partial / \partial q^{i}\right\}$ en $T Q$ y $\left\{\mathrm{d} q^{i}\right\}$ en $T^{*} Q$. Denotaremos por $p$ y $\bar{p}$ la matriz de cambio de marcos y su inversa, respectivamente, es decir

$$
\frac{\partial}{\partial q^{k}}=p_{k}^{l} E_{l}, \quad E_{k}=\bar{p}_{k}^{l} \frac{\partial}{\partial q^{l}}
$$

y sus transpuestas asociadas

$$
\mathrm{d} q^{l}=\bar{p}_{k}^{l} E^{k}, \quad E^{l}=p_{k}^{l} \mathrm{~d} q^{k} .
$$

Tomemos entonces una curva $c:(-\varepsilon, \varepsilon) \rightarrow T^{*} Q$ tal que

$$
c(s)=c_{i}(s) E^{i}, \quad c(0)=\alpha=\alpha_{i} E^{i}, \quad q^{\prime}(0)=X=X^{i} E_{i}, \quad \frac{D c}{D s}=0,
$$

donde $q(s):=(\pi \circ c)(s)$ y $q(0)=q:=\pi(\alpha)$. La última ecuación dice que la curva $c$ es horizontal respecto de $\nabla$ y usando los símbolos de Christoffel respecto de los marcos adaptados [ver (1.2)], esto implica que

$$
c_{i}^{\prime}(s)=-\Gamma_{j i}^{k}(q(s)) c_{k}(s) \hat{X}^{j}(q(s)),
$$

donde $\hat{X}$ es un campo vectorial que extiende a $X$ en un entorno de $q=\pi(\alpha)$. Por lo tanto

$$
\begin{aligned}
\left\langle\mathbb{B}\left(\mathfrak{d} \circ p_{V}\right)(\alpha), X\right\rangle & =\left.\frac{\mathrm{d}}{\mathrm{d} s}\right|_{s=0}\left(\mathfrak{d} \circ p_{V}\right)(c(s))=\left.\frac{1}{2} \frac{\mathrm{d}}{\mathrm{d} s}\right|_{s=0}\left[c_{\mu}(s) \delta^{\mu \nu}(q(s)) c_{\nu}(s)\right] \\
& =c_{\mu}^{\prime}(0) \delta^{\mu \nu}(q) \sigma_{\nu}+\frac{1}{2} \alpha_{\mu} \frac{\partial \delta^{\mu \nu}}{\partial q^{k}}(q) \bar{p}_{j}^{k}(q) X^{j} \alpha_{\nu} \\
& =-\Gamma_{j \mu}^{k}(q) \alpha_{k} X^{j}(q) \delta^{\mu \nu}(q) \alpha_{\nu}+\frac{1}{2} \alpha_{\mu} \frac{\partial \delta^{\mu \nu}}{\partial q^{k}}(q) \bar{p}_{j}^{k}(q) X^{j} \alpha_{\nu}
\end{aligned}
$$

luego, tomando $X=\mathbb{F h}(\alpha)=\rho^{\sharp}(\alpha)$, tenemos

$$
\left\langle\mathbb{B}\left(\mathfrak{d} \circ p_{V}\right)(\alpha), \mathbb{F} \mathfrak{h}(\alpha)\right\rangle=\frac{1}{2} \check{\mathbb{H}}^{j l}(q) \bar{p}_{j}^{k}(q) \frac{\partial \delta^{\mu \nu}}{\partial q^{k}}(q) \alpha_{l} \alpha_{\mu} \alpha_{\nu}-\Gamma_{j \mu}^{k}(q) \check{\mathbb{H}}^{j l}(q) \delta^{\mu \nu}(q) \alpha_{\nu} \alpha_{l} \alpha_{k},
$$

donde

$$
\check{\mathbb{H}}^{i j}=\rho\left(E^{i}, E^{j}\right) .
$$


Por otra parte

$$
\begin{aligned}
\langle\mathbb{B} \mathfrak{h}(\alpha), X\rangle & =\left.\frac{\mathrm{d}}{\mathrm{d} s}\right|_{s=0} \mathfrak{h}(c(s))=\left.\frac{1}{2} \frac{\mathrm{d}}{\mathrm{d} s}\right|_{s=0}\left(c_{i}(s) \check{\mathbb{H}}^{i j}(q(s)) c_{j}(s)\right) \\
& =\frac{1}{2} \alpha_{i} \frac{\partial \check{\mathbb{H}}^{i j}}{\partial q^{k}}(q) \alpha_{j} \bar{p}_{l}^{k} X^{l}-\Gamma_{l j}^{k}(q) \alpha_{k} X^{l} \check{\mathbb{H}}^{j i}(q) \alpha_{i},
\end{aligned}
$$

luego

$$
\mathbb{B h}(\alpha)=\left(\frac{1}{2} \frac{\partial \check{\mathbb{H}}^{i j}}{\partial q^{k}}(q) \bar{p}_{l}^{k} \alpha_{i} \alpha_{j}-\Gamma_{l j}^{k}(q) \check{\mathbb{H}}^{j i}(q) \alpha_{k} \alpha_{i}\right) E^{l}
$$

por lo que

$$
p_{V}(\mathbb{B h}(\alpha))=\left(\frac{1}{2} \frac{\partial \check{\Pi}^{i j}}{\partial q^{k}}(q) \bar{p}_{\mu}^{k} \alpha_{i} \alpha_{j}-\Gamma_{\mu j}^{k}(q) \check{H}^{j i}(q) \alpha_{k} \alpha_{i}\right) E^{\mu}
$$

Por lo tanto

$$
\left\langle p_{V}(\mathbb{B} \mathfrak{h}(\alpha)), \mathbb{F} \mathfrak{d}\left(p_{V}(\alpha)\right)\right\rangle=\left(\frac{1}{2} \frac{\partial \check{\mathbb{H}}^{i j}}{\partial q^{k}}(q) \bar{p}_{\mu}^{k} \alpha_{i} \alpha_{j}-\Gamma_{\mu j}^{k}(q) \check{\mathbb{H}}^{j i}(q) \alpha_{k} \alpha_{i}\right) \delta^{\mu \nu}(q) \alpha_{\nu}
$$

Juntando las expresiones (4.13) y (4.14), la versión en coordenadas de las ecuaciones cinéticas resulta

$$
\check{\mathbb{H}}^{j i}(q) \bar{p}_{j}^{k}(q) \frac{\partial \delta^{\mu \nu}}{\partial q^{k}}(q) \sigma_{i} \sigma_{\mu} \sigma_{\nu}=\left[\bar{p}_{\mu}^{k} \frac{\partial \check{\mathbb{H}}^{i j}}{\partial q^{k}}(q)+2\left(\Gamma_{k \mu}^{j}(q)-\Gamma_{\mu k}^{j}(q)\right) \check{\mathbb{H}}^{k i}(q)\right] \delta^{\mu \nu}(q) \sigma_{\nu} \sigma_{i} \sigma_{j} .
$$

Dado que la conexión que estamos considerando es sin torsión,

$$
\Gamma_{i j}^{k}-\Gamma_{j i}^{k}=\frac{\partial p_{l}^{k}}{\partial q^{m}}\left[\bar{p}_{i}^{l} \bar{p}_{j}^{m}-\bar{p}_{j}^{l} \bar{p}_{i}^{m}\right]
$$

lo cual refleja que la ecuación es independiente de la conexión.

Para simplificar aun más la expresión (4.15), recordemos que $\alpha=\gamma_{-}(\lambda)$, por lo que $\alpha_{\tau}=\lambda_{\tau} \mathrm{y}$ $\alpha_{s+a}=-\gamma_{a}^{\tau} \lambda_{\tau}$. Luego, definiendo

$$
\begin{gathered}
\tilde{B}_{\theta}^{i j}=\bar{p}_{\theta}^{k} \frac{\partial \check{\mathbb{H}}^{i j}}{\partial q^{k}}+2\left(\Gamma_{k \theta}^{j}-\Gamma_{\theta k}^{j}\right) \check{\mathbb{H}}^{k i} \\
B_{\theta}^{\mu \nu}=\tilde{B}_{\theta}^{\mu \nu}-\gamma_{a}^{\mu} \tilde{B}_{\theta}^{s+a, \nu}-\tilde{B}_{\theta}^{\mu, s+b} \gamma_{b}^{\nu}+\gamma_{a}^{\mu} B_{\theta}^{s+a, s+b} \gamma_{b}^{\nu}
\end{gathered}
$$

y

$$
A^{\tau k}:=\left(\check{\mathbb{H}}^{j \tau}-\check{\mathbb{H}}^{j, s+a} \gamma_{a}^{\tau}\right) \bar{p}_{j}^{k}
$$

podemos escribir

$$
\left(A^{\tau k}(q) \frac{\partial \delta^{\mu \nu}}{\partial q^{k}}(q)-B_{\theta}^{\mu \nu}(q) \delta^{\theta \tau}(q)\right) \lambda_{\tau} \lambda_{\mu} \lambda_{\nu}=0,
$$

para todo $\left(\lambda_{1}, \ldots, \lambda_{s}\right) \in \mathbb{R}^{s}$. Por lo tanto, las ecuaciones cinéticas se escriben (omitimos el punto de evaluación $q$ para simplificar)

$$
\sum_{(\mu, \nu, \tau)}\left(A^{\tau k} \frac{\partial \delta^{\mu \nu}}{\partial q^{k}}-B_{\theta}^{\mu \nu} \delta^{\theta \tau}\right)=0, \quad \mu \nu \tau=1, \ldots, s,
$$

donde $\sum_{(\mu, \nu, \tau)}$ denota suma sobre todas las posibles permutaciones de los índices $\mu, \nu, \tau$. 
En el caso de las ecuaciones potenciales, haciendo un cálculo similar al anterior, encontramos

$$
\left[\left(\check{\mathbb{H}}^{j \tau}-\check{\mathbb{H}}^{j, s+a} \gamma_{a}^{\tau}\right) \bar{p}_{j}^{k} \frac{\partial v}{\partial q^{k}}(q)-\frac{\partial h}{\partial q^{k}}(q) \bar{p}_{\mu}^{k} \delta^{\mu \tau}\right] \lambda_{\tau}=0
$$

para todo $\left(\lambda_{1}, \ldots, \lambda_{s}\right) \in \mathbb{R}^{s}$. Por lo tanto, siguiendo (4.19), las ecuaciones potenciales se escriben

$$
A^{\tau k} \frac{\partial v}{\partial q^{k}}-\frac{\partial h}{\partial q^{k}} \bar{p}_{\mu}^{k} \delta^{\mu \tau}=0, \quad \tau=1, \ldots, s .
$$

Observación 4.7.

1. Las ecuaciones (4.20) y (4.21) constituyen una generalización de las ecuaciones (3.12) y (3.15) y son válidas para sistemas con $W$ no necesariamente integrable.

2. A diferencia del caso regularmente actuado, los números $B_{\theta}^{\mu \nu}$ en (4.18) no son simétricos en los índices $\mu, \nu$ debido a la presencia del término (4.16). Es fácil ver que si los marcos $E_{i}$ conmutan dos a dos (por ejemplo en el caso del marco coordenado), entonces dicho término se anula y $B_{\theta}^{\mu \nu}$ es efectivamente simétrico en esos índices. En tal caso la suma $\sum_{(\mu, \nu, \tau)}$ en (4.20) puede hacerse sobre permutaciones cíclicas únicamente.

3. Si $W$ es integrable, es posible escoger coordenadas adaptadas a $W$, i.e. coordenadas en las cuales $W_{q}=\operatorname{span}\left\{\mathrm{d} q^{s+a}\right\}_{a=1}^{m}$. Para este tipo de espacios de actuación, podemos tomar como complemento local

$$
V_{q}=\operatorname{span}\left\{\mathrm{d} q^{\mu}\right\}_{\mu=1}^{s} .
$$

Si además consideramos como marcos adaptados a los marcos coordenados asociados a estas coordenadas, la matriz de cambio de marcos $p$ y su inversa $\bar{p}$ son ambas la identidad. Luego, los números (4.18) y (4.19) coinciden con (3.10) y (3.9), respectivamente [ver (4.17)]. De este modo reobtenemos el caso regularmente actuado.

La ventaja de esta generalización de las ecuaciones cinéticas y potenciales para sistemas regularmente actuados, es que (4.20) y (4.21) son válidas en coordenadas arbitrarias y para un subfibrado $W$ también arbitrario, una vez fijado el complemento $V$ y el sistema de marcos adaptados $E^{i}$, que a su vez pueden elegirse libremente. Por el contrario, en el caso regularmente actuado, las ecuaciones (3.12) y (3.15) son válidas únicamente en coordenadas que rectifican al subfibrado $W$, que por lo tanto debe ser integrable.

En la sección siguiente explotaremos este formalismo para encontrar soluciones locales de las ecuaciones cinéticas y potenciales. En primer lugar, encontraremos soluciones de las ecuaciones potenciales para sistemas con grado actuación genérico suponiendo que tenemos una solución de las Ecuaciones (4.20). Seguidamente, para sistemas 1-subactuados, construiremos también soluciones de (4.20). Si bien ya vimos en el capítulo anterior que para estos últimos sistemas es posible dar condiciones necesarias y suficientes para la existencia de soluciones locales definidas positivas (ver Teoremas 3.1 y 3.2), el 
4. Soluciones por cuadraturas de las ecuaciones cinéticas y potenciales para sistemas 1-subactuados

objetivo de la próxima sección es otro, a saber, dar un procedimiento sistemático para construir soluciones explícitas de las ecuaciones. En particular, mostraremos que en algunos casos es posible encontrar soluciones por cuadraturas, i.e. soluciones que pueden calcularse computando integrales ordinarias de funciones conocidas.

\subsection{Integración por cuadraturas de las ecuaciones potenciales: actuación arbitraria}

Sea $(H, \mathcal{W})$ un sistema Hamltoniano subactuado con $H$ simple $\mathcal{W}$ dado por un subfibrado vectorial $W \subset T^{*} Q$. Siguiendo el formalismo de la sección anterior, supongamos que $V \subset T^{*} Q$ es un subfibrado vectorial tal que $T^{*} Q=V \oplus W$. Fijemos marcos adaptados a la descomposición y consideremos las Ecuaciones (4.8) y (4.11).

Dado que los términos que aparecen en (4.8) son lineales, basta ver que dicha ecuación se cumple para cada sección $E^{\mu}$, es decir

$$
\left\langle\mathrm{d} v(q), \mathbb{F} \mathfrak{h}\left(\gamma_{-}\left(E^{\mu}\right)\right)\right\rangle=\left\langle p_{V}(\mathrm{~d} h(q)), \mathbb{F} \mathfrak{d}\left(E^{\mu}\right)\right\rangle, \quad \mu=1, \ldots, s .
$$

que a su vez se puede escribir

$$
X^{\mu}(v)=f^{\mu}, \quad \mu=1, \ldots, s .
$$

donde

$$
X^{\mu}=\mathbb{F} \mathfrak{h}\left(\gamma_{-}\left(E^{\mu}\right)\right)=\rho^{\sharp}\left(\gamma_{-}\left(E^{\mu}\right)\right)
$$

y

$$
f^{\mu}=\left\langle p_{V}(\mathrm{~d} h(q)), \mathbb{F} \mathfrak{d}\left(E^{\mu}\right)\right\rangle .
$$

Es sencillo ver que los campos $X^{\mu}$ son linealmente independientes. Luego, si $\left[X^{\mu}, X^{\nu}\right]=0$ para todo $\mu, \nu=1, \ldots, s$, sabemos que es posible encontrar coordenadas $(U, \varphi)$ que rectifiquen dichos campos y en tal caso las ecuaciones potenciales se escribirían

$$
\frac{\partial v}{\partial q^{\mu}}=f^{\mu}
$$

cuya solución puede ser encontrada calculando integrales ordinarias de funciones relacionadas con la ecuación.

Observación 4.8. Tomando una carta coordenada $(U, \varphi)$, la expresión local de los campos $X^{\mu}$ es

$$
X^{\mu}=\left(\check{\mathbb{H}}^{j \mu}-\check{\mathbb{H}}^{j, s+a} \gamma_{a}^{\mu}\right) \bar{p}_{j}^{k} \frac{\partial}{\partial q^{k}},
$$

donde $\check{H}^{i j}$ y $\gamma_{a}^{\mu}$ son los coeficientes de las representaciones matriciales de $\rho^{\sharp} \mathrm{y} \gamma$ respecto de los marcos adaptados. En el caso en el cual las coordenadas rectifiquen a los campos $X^{\mu}$, tenemos que

$$
\left(\check{\mathbb{H}}^{j \mu}-\check{\mathbb{H}}^{j, s+a} \gamma_{a}^{\mu}\right) \bar{p}_{j}^{k}=\delta^{\mu k},
$$

donde $\delta^{\mu k}$ es símbolo de Kronecker. 


\subsubsection{Elección del morfismo $\gamma$}

Basándonos en el razonamiento anterior, lo que mostraremos a continuación es que, dada una carta coordenada $(U, \varphi)$ es posible construir algebraicamente un complemento local $V$ de $W$, marcos $E^{i}$ adaptados a la descomposición y un morfismo $\gamma \in L(V, W)$ de manera tal que los campos (4.22) coinciden con los primeros $s$ campos coordenados. Primero tenemos el siguiente resultado que es de fácil demostración.

Lema 4.4. Sea $W \subset T^{*} Q$ un subfibrado vectorial de rango m y sea $\left(\tilde{U},\left(\tilde{q}^{1}, \ldots, \tilde{q}^{n}\right)\right)$ una carta coordenada de $Q$ que contiene a $\bar{q}$. Entonces existe una matriz invertible constante $C$ y un abierto $\bar{q} \in U \subseteq \tilde{U}$ tal que, definiendo $q^{j}:=C_{i}^{j} \tilde{q}^{i}$, la carta coordenada $\left(U,\left(q^{1}, \ldots, q^{n}\right)\right)$ cumple

$$
W_{\bar{q}}=\left\{\left.\mathrm{d} q^{s+a}\right|_{\bar{q}}\right\}_{a=1}^{m} .
$$

Observación 4.9. La construcción de las coordenadas $\left(U,\left(q^{1}, \ldots, q^{n}\right)\right)$ del lema anterior se realiza de manera puramente algebraica a partir de coordenadas locales arbitrarias y utilizando herramientas de álgebra lineal.

Teorema 4.1. Sea $W \subset T^{*} Q$ un subfibrado vectorial de rango my sea $\left(U,\left(q^{1}, \ldots, q^{n}\right)\right)$ una carta coordenada de $Q$ como la del Lema 4.4. Entonces existen un subfibrado vectorial $V \subset T^{*} Q$ tal que $T_{q}^{*} Q=V_{q} \oplus W_{q}$ para todo $q \in U$, marcos locales sobre $U$ adaptados a esta descomposición y un morfismo de fibrados vectoriales $\gamma \in L(V, W)$ tales que [ver (4.22)]

$$
X^{\mu}=\frac{\partial}{\partial q^{\mu}}
$$

Demostración. En primer lugar, denotemos $W^{\sharp}=\rho^{\sharp}(W) \subset T Q$ y tomemos un complemento $V^{\sharp} \subset$ $T Q$ de $W^{\sharp}$ sobre $U$, i.e. un subfibrado $V^{\sharp}$ tal que $T Q=V^{\sharp} \oplus W^{\sharp}$ sobre $U$. Dado que $\rho^{b}$ es un isomorfismo de fibrados vectoriales, podemos definir $V:=\rho^{b}\left(V^{\sharp}\right)$ y es claro entonces que $T^{*} Q=V \oplus W$ sobre $U$.

Por otro lado, gracias a las características de las coordenadas, es claro que el espacio $W^{\sharp}$ está generado en $\bar{q}$ por los campos

$$
\rho^{\sharp}\left(\left.\mathrm{d} q^{s+a}\right|_{\bar{q}}\right), \quad a=1, \ldots, m .
$$

Es sencillo ver entonces que el espacio

$$
A_{q}:=\operatorname{span}\left\{\left.\frac{\partial}{\partial q^{\mu}}\right|_{q}\right\}_{\mu=1}^{s}
$$

satisface $A_{\bar{q}} \cap W_{\bar{q}}^{\sharp}=\{0\}$ y por continuidad podemos suponer que

$$
A_{q} \cap W_{q}^{\sharp}=\{0\}, \quad \forall q \in U .
$$

En base a la descomposición $T Q=V^{\sharp} \oplus W^{\sharp}$, cada campo coordenado se escribe

$$
\frac{\partial}{\partial q^{\mu}}=X_{\mu}^{V}+X_{\mu}^{W}
$$


4. Soluciones por cuadraturas de las ecuaciones cinéticas y potenciales para sistemas 1-subactuados

donde $X_{\mu}^{V} \in V^{\sharp}$ y $X_{\mu}^{W} \in W^{\sharp}$. Si denotamos $p_{V^{\sharp}}: T Q \rightarrow V^{\sharp}$ a la proyección canónica, dado que los campos $\partial / \partial q^{\mu}$ son linealmente independientes basta ver que $\left.p_{V^{\sharp}}\right|_{A}$ es inyectivo para concluir que los campos $X_{\mu}^{V}$ son también linealmente independientes. Pero [ver (4.27)]

$$
\operatorname{ker}\left(\left.p_{V^{\sharp}}\right|_{A}\right)=\operatorname{ker} p_{V^{\sharp}} \cap A=W^{\sharp} \cap A=\{0\},
$$

de donde concluimos que $\left.p_{V^{\sharp}}\right|_{A}$ es inyectivo y que los campos $X_{\mu}^{V}$ son linealmente independientes. Esto dice que $V^{\sharp}=\operatorname{span}\left\{X_{1}^{V}, \ldots, X_{s}^{V}\right\}$, y podemos tomar

$$
E^{\mu}=\rho^{b}\left(X_{\mu}^{V}\right)=\rho^{b}\left(p_{V^{\sharp}}\left(\frac{\partial}{\partial q^{\mu}}\right)\right)=p_{V}\left(\rho^{b}\left(\frac{\partial}{\partial q^{\mu}}\right)\right),
$$

como marco local en $V$. A su vez, podemos extender este marco con un marco local $E^{s+1}, \ldots, E^{n}$ de $W$ a un marco de $T^{*} Q$.

En estas condiciones, podemos definir localmente un morfismo de fibrados vectoriales $\tilde{\gamma}: V^{\sharp} \rightarrow$ $W^{\sharp}$ por sus valores en un marco de $V^{\sharp}$ de la siguiente manera

$$
\tilde{\gamma}\left(X_{\mu}^{V}\right)=\frac{\partial}{\partial q^{\mu}}-X_{\mu}^{V}
$$

A partir de este morfismo, definimos

$$
\gamma=-\rho^{b} \circ \tilde{\gamma} \circ \rho^{\sharp}
$$

Con esta definición,

$$
\begin{aligned}
\rho^{\sharp}\left(\gamma_{-}\left(E^{\mu}\right)\right) & =\rho^{\sharp}\left(E^{\mu}-\gamma\left(E^{\mu}\right)\right)=\rho^{\sharp}\left[\rho^{b}\left(X_{\mu}^{V}\right)+\left(\rho^{b} \circ \tilde{\gamma} \circ \rho^{\sharp}\right)\left(\rho^{b}\left(X_{\mu}^{V}\right)\right)\right] \\
& =X_{\mu}^{V}+\tilde{\gamma}\left(X_{\mu}^{V}\right)=X_{\mu}^{V}+\frac{\partial}{\partial q^{\mu}}-X_{\mu}^{V}=\frac{\partial}{\partial q^{\mu}} .
\end{aligned}
$$

Observación 4.10. En particular, tomando $V^{\sharp}=A$ es claro que

$$
p_{V^{\sharp}}\left(\frac{\partial}{\partial q^{\mu}}\right)=\frac{\partial}{\partial q^{\mu}},
$$

de modo que $\tilde{\gamma}=\gamma=0$.

\subsubsection{Condiciones de integrabilidad de las ecuaciones potenciales}

Siguiendo la construcción del Teorema 4.1, fijemos un complemento arbitrario $V^{\sharp}$ de $W^{\sharp}=\rho^{\sharp}(W)$ y definamos $E^{\mu}$ por (4.28) y $\gamma$ por (4.29) y (4.30). Fijemos también coordenadas $(U, \varphi)$ sobre $Q$. En estas condiciones, según (4.24) y (4.25), las ecuaciones (4.20) se escriben

$$
\sum_{(\mu, \nu, \sigma)}\left(\frac{\partial \delta^{\mu \nu}}{\partial q^{\tau}}-B_{\theta}^{\mu \nu} \delta^{\theta \sigma}\right)=0, \quad \mu, \nu, \sigma=1, \ldots, s
$$


mientras que (4.21) resulta

$$
\frac{\partial v}{\partial q^{\mu}}=f^{\mu}, \quad \mu=1, \ldots, s
$$

donde [recordar (4.23)]

$$
f^{\mu}=\delta^{\mu \tau} \bar{p}_{\tau}^{k} \frac{\partial h}{\partial q^{k}}
$$

Más aun, si estas condiciones se cumplen, es posible hallar $v$ a menos de cuadraturas.

Supongamos ahora que $\delta$ es una solución de las ecuaciones (4.31). Es bien sabido que una condición necesaria y suficiente para la existencia de soluciones de (4.32) es

$$
\frac{\partial f^{\mu}}{\partial q^{\nu}}=\frac{\partial f^{\nu}}{\partial q^{\mu}}, \quad \forall \mu, \nu=1, \ldots, s .
$$

Observación 4.11. Notemos que (4.34) es un nuevo conjunto de PDEs para el tensor $\delta$ [ver (4.33)]

Por otro lado, puede mostrarse que si fijamos una condición de borde sobre el conjunto

$$
S:=\varphi(U) \cap\left(\{(\underbrace{0, \ldots, 0}_{s})\} \times \mathbb{R}^{m}\right),
$$

entonces existe una única solución de (4.32) cumpliendo tal condición.

Observación 4.12. Vale la pena notar que, en el caso de subactuación 1 (o sea, el caso $s=1$ ), las ecuaciones (4.34) se reducen a una única ecuación para $\mu=\nu=1$, que se cumple trivialmente. Por lo tanto, si tenemos una solución $\delta$ para (4.31), el argumento anterior nos asegura que, al menos para el caso de sistemas 1-subactuados, existe una solución de la (única) ecuación potencial (como ya habíamos visto en el Capítulo 3). Más aun, tal solución puede encontrarase por cuadraturas, i.e. calculando integrales ordinarias. Estudiaremos esta estrategia en la Sección $\$ 4.3$.

\subsubsection{Condiciones de positividad}

Supongamos ahora que (4.34) se cumple y sea $v$ una solución de (4.32). En estas condiciones, la función $v$ será definida positiva alrededor de $\mathbf{0}$ si y sólo si $v$ tiene un mínimo local no degenerado en $\mathbf{0}$ $\mathrm{y}$, como $v$ es suave, tenemos que:

1. 0 debe ser un punto crítico de $v$,

2. la matriz Hessiana de $v$ en $\mathbf{0}$ debe ser definida positiva.

Veamos cómo se traducen estas condiciones en términos de $h, \mathfrak{h}$ y $\delta$. Supongamos que $\mathbf{0}$ es crítico para $h$, i.e. $\operatorname{grad} h(\mathbf{0})=0$. Si escogemos $v$ de modo que, sobre el conjunto $S$ [ver Ec. (4.35)], se cumpla

$$
v\left(0, \ldots, 0, s^{1}, \ldots, s^{m}\right)=\kappa_{a}\left(s^{a}\right)^{2},
$$


4. Soluciones por cuadraturas de las ecuaciones cinéticas y potenciales para sistemas 1-subactuados

donde $\kappa_{1}, \ldots, \kappa_{m}$ son constantes, se sigue de (4.32) y de las condiciones de borde que $\operatorname{grad} v(\mathbf{0})=0$. Por lo tanto, como exige el punto 1 , el término potencial $v$ es crítico en $\mathbf{0}$. Estudiemos ahora la matriz Hessiana de $v$ en el origen. Notemos que, usando la Ec. (4.32) y le hecho de que $h$ es crítico en $\mathbf{0}$,

$$
\operatorname{Hess}(v)_{\mu \nu}(\mathbf{0})=\frac{\partial f^{\mu}}{\partial q^{\nu}}(\mathbf{0})=\left[\delta^{\mu \tau} \bar{p}_{\tau}^{k} \frac{\partial^{2} h}{\partial q^{k} \partial q^{\nu}}\right](\mathbf{0})=\left[\delta^{\mu \tau} \bar{p}_{\tau}^{k} \operatorname{Hess}(h)_{k \nu}\right](\mathbf{0}), \quad \forall 1 \leq \mu, \nu \leq s .
$$

Impongamos ahora la siguiente condición de borde sobre $S$ :

$$
v\left(0, \ldots, 0, s^{1}, \ldots, s^{m}\right)=\frac{\kappa}{2} \sum_{a=1}^{m}\left(s^{a}\right)^{2},
$$

con $\kappa>0$. Usando esto, tenemos que

$$
\operatorname{Hess}(v)(\mathbf{0})=\left[\begin{array}{cc}
\mathbb{M} & \mathbb{A} \\
\mathbb{A}^{t} & \kappa \mathbb{I}_{m}
\end{array}\right],
$$

donde $\mathbb{I}_{m}$ es la matriz identidad de $m \times m$, la matriz $\mathbb{A}$ es de $s \times m$ con entradas

$$
\mathbb{A}_{\mu a}:=\frac{\partial f^{\mu}}{\partial q^{s+a}}(\mathbf{0})=\left[\delta^{\mu \tau} \bar{p}_{\tau}^{k} \operatorname{Hess}(h)_{k, s+a}\right](\mathbf{0}), \quad \forall 1 \leq \mu \leq s, 1 \leq a \leq m,
$$

y la matriz $\mathbb{M}$ es de $s \times s$ y sus entradas están dadas por [ver Ec. (4.36)]

$$
\mathbb{M}_{\mu \nu}:=\left[\delta^{\mu \tau} \bar{p}_{\tau}^{k} \operatorname{Hess}(h)_{k \nu}\right](\mathbf{0})
$$

En esta situación es claro que, si $\operatorname{Hess}(v)(\mathbf{0})$ es definida positiva, entonces también lo es $\mathbb{M}$ (esto es consecuencia del Teorema A.1). Veamos ahora que también vale la afirmación recíproca.

Observación 4.13. Notemos que tanto $\mathbb{A}$ como $\mathbb{M}$ están completamente determinadas por $\delta, h$ y las coordenadas escogidas; de manera que $\kappa$ puede escogerse idependientemente de ellas.

Proposición 4.4. Sea $v$ una solución local de (4.32) cumpliendo la condición de borde (4.37) y supongamos que la matriz M, dada por (4.39), es definida positiva. Entonces, existe una constante $\kappa$ tal que Hess $(v)(\mathbf{0})$ es definida positiva.

Demostración. Supongamos que $\mathbb{M}$ es definida positiva y tomemos $(\vec{u}, \vec{w}) \neq \mathbf{0}$ con $\vec{u} \in \mathbb{R}^{s}$ y $\vec{w} \in \mathbb{R}^{m}$. Entonces

$$
\begin{aligned}
(\vec{u}, \vec{w})\left[\begin{array}{cc}
\mathbb{M} & \mathbb{A} \\
\mathbb{A}^{t} & \kappa \mathbb{I}
\end{array}\right]\left[\begin{array}{l}
\vec{u}^{t} \\
\vec{w}^{t}
\end{array}\right] & =\vec{u} \mathbb{M} \vec{u}^{t}+2 \vec{u} \mathbb{A} \vec{w}^{t}+\kappa\|\vec{w}\|^{2} \\
& =\|\vec{u}\|_{\mathbb{M}}^{2}+2 \vec{u} \mathbb{A} \vec{w}^{t}+\kappa\|\vec{w}\|^{2} \\
& \geq\|\vec{u}\|_{\mathbb{M}}^{2}-2\|\vec{u}\|\|\mathbb{A}\|\|\vec{w}\|+\kappa\|\vec{w}\|^{2}
\end{aligned}
$$

donde $\|\cdot\|$ denota la norma euclídea en espacio euclídeo que corresponda, y $\|\cdot\|_{\mathbb{M}}$ es la norma asociada con $\mathbb{M}$. Si $\mathbb{A}$ es la matriz nula, entonces $\operatorname{Hess}(v)(\mathbf{0})$ es claramente definida positiva. Supongamos ahora 
que $\mathbb{A}$ es no nula. Dado que cualquier norma en un espacio euclídeo es equivalente a la norma euclídea, existe una constante positiva $\alpha$ tal que

$$
\|\vec{u}\|_{\mathbb{M}} \geq \alpha\|\vec{u}\|, \quad \forall \vec{u} \in \mathbb{R}^{s} .
$$

La constante $\alpha$ puede calcularse del siguiente modo:

$$
\alpha=\min _{\|\vec{u}\|=1} \sqrt{\vec{u} \mathbb{M} \vec{u}^{t}}=\sqrt{\lambda_{\text {mín }}^{\mathbb{M}}}
$$

donde $\lambda_{\text {mín }}^{\mathbb{M}}$ es el mínimo autovalor de $\mathbb{M}$. Luego,

$$
\begin{aligned}
(\vec{u}, \vec{w})\left[\begin{array}{cc}
\mathbb{M} & \mathbb{A} \\
\mathbb{A}^{t} & \kappa \mathbb{I}
\end{array}\right]\left[\begin{array}{c}
\vec{u}^{t} \\
\vec{w}^{t}
\end{array}\right] & \geq \alpha^{2}\|\vec{u}\|^{2}-2\|\vec{u}\|\|\mathbb{A}\|\|\vec{w}\|+\kappa\|\vec{w}\|^{2} \\
& =\alpha^{2}\left(\|\vec{u}\|^{2}-2\|\vec{u}\|\|\mathbb{A}\| \frac{\|\vec{w}\|}{\alpha^{2}}+\kappa \frac{\|\vec{w}\|^{2}}{\alpha^{2}}\right) .
\end{aligned}
$$

Definiendo $\beta=\frac{\|\mathbb{A}\|}{\alpha}$, tenemos que

$$
\begin{aligned}
(\vec{u}, \vec{w})\left[\begin{array}{cc}
\mathbb{M} & \mathbb{A} \\
\mathbb{A}^{t} & \kappa \mathbb{I}
\end{array}\right]\left[\begin{array}{c}
\vec{u}^{t} \\
\vec{w}^{t}
\end{array}\right] & \geq \alpha^{2} \beta^{2}\left(\frac{\|\vec{u}\|^{2}}{\beta^{2}}-2 \frac{\|\vec{w}\|}{\alpha} \frac{\|\vec{u}\|}{\beta}+\frac{\kappa}{\beta^{2}} \frac{\|\vec{w}\|^{2}}{\alpha^{2}}\right) \\
& =\alpha^{2} \beta^{2}\left[\left(\frac{\kappa}{\beta^{2}}-1\right)\left(\frac{\|\vec{w}\|}{\alpha}\right)^{2}+\left(\frac{\|\vec{w}\|}{\alpha}-\frac{\|\vec{u}\|}{\beta}\right)^{2}\right] .
\end{aligned}
$$

Por lo tanto, si escogemos $\kappa>\beta^{2}$ (ver la última Observación), i.e.

$$
\kappa>\left(\frac{\|\mathbb{A}\|}{\alpha}\right)^{2},
$$

se sigue que $\operatorname{Hess}(v)(\mathbf{0})$ es definida positiva.

En otras palabras, la proposición anterior dice que, si $\mathbb{M}$ es una matriz definida positiva, también lo es $\operatorname{Hess}(v)(\mathbf{0})$, siempre que $\kappa$ se tome suficientemente grande.

En conclusión, si $h$ es crítico en $\mathbf{0}, \mathbb{M}$ es definida positiva, y además se cumple (4.34), con lo cual existe una solución local $v$ de (4.32) alrededor de $\mathbf{0}$, entonces es posible escoger condiciones de borde tales que $v$ resulta definida positiva alrededor de $\mathbf{0}$.

\subsubsection{Construcción por cuadraturas de soluciones locales}

Los pasos desarrollados en las últimas secciones dan origen a un procedimiento concreto para construir una solución local de las ecuaciones potenciales que es definida positiva y además es computable por cuadraturas. Más precisamente, tenemos el siguiente procedimiento de construcción:

1. tomar coordenadas $(U, \varphi)$ como las del Lema 4.4; 
4. Soluciones por cuadraturas de las ecuaciones cinéticas y potenciales para sistemas 1-subactuados

2. escoger un complemento $V$ de $W$ (o bien $V^{\sharp}$ de $W^{\sharp}$ ), marcos adaptados $E^{1}, \ldots, E^{n}$ y un morfismo $\gamma$ como los del Teorema 4.1;

3. tomar una solución $\delta$ de (4.31);

4. chequear que alrededor de $\mathbf{0}$ valen las ecuaciones [ver (4.34)]

$$
\frac{\partial f^{\mu}}{\partial q^{\nu}}=\frac{\partial f^{\nu}}{\partial q^{\mu}}, \quad \forall \mu, \nu=1, \ldots, s
$$

donde $f^{\mu}$ está dada por (4.33).

5. definir $v \operatorname{como}^{3}$

$$
v\left(q^{1}, \ldots, q^{n}\right):=\sum_{\mu=1}^{s} \int_{0}^{q^{\mu}} f^{\mu}\left(0, \ldots, 0, t, q^{\mu+1}, \ldots, q^{n}\right) \mathrm{d} t+\frac{\kappa}{2} \sum_{a=1}^{m}\left(q^{s+a}\right)^{2}
$$

para alguna constante $\kappa$ [es fácil ver que esta expresión integra la Ecuación (4.32)];

6. verificar que la matriz $\mathbb{M}$ dada por (4.39) es definida positiva;

7. escoger $\kappa$ de manera que [recordar Ecs. (4.38), (4.40) and (4.41)]

$$
\kappa>\frac{\sum_{b=1}^{m} \sum_{\mu=1}^{s}\left(\partial f^{\mu} / \partial q^{s+b}(\mathbf{0})\right)^{2}}{\lambda_{\text {mín }}^{\mathbb{M}}} .
$$

Dado que la elección de las coordenadas es arbitraria (a menos de una construcción algebraica) y que la construcción de los objetos mencionados en el punto 2 es también algebraica, concluimos que el procedimiento anterior permite construir la solución del punto 5 por cuadraturas. En consecuencia, hemos probado el siguiente teorema.

Teorema 4.2 (Construcción por cuadraturas de soluciones de las ecuaciones potenciales). Sea $(U, \varphi)$ una carta coordenada como la del Lema 4.4 y escojamos un complemento $V$, marcos adaptados $E^{1}, \ldots, E^{n}$ y un morfismo $\gamma$ como los del Teorema 4.1. Fijemos también una solución $\delta$ de las ecuaciones (4.31). Luego, si

$$
\frac{\partial f^{\tau}}{\partial q^{\sigma}}=\frac{\partial f^{\sigma}}{\partial q^{\tau}}, \quad \forall \tau, \sigma=1, \ldots, s ;
$$

entonces existe una solución local $v$ de (4.32) [y por lo tanto de (4.8)] que puede determinarse explícitamente a menos cuadraturas (i.e. a menos de primitivas de funciones asociadas a las ecuaciones involucradas). Si además la matriz [ver Ec. (4.39)] M es definida positiva, dicha solución puede escogerse definida positiva alrededor de $\mathbf{0}$. Más aun, la solución puede construirse siguiendo los pasos del 1 al 7 en el procedimiento de arriba.

\footnotetext{
${ }^{3}$ Aquí estamos suponiendo, sin pérdida de generalidad, que el entorno $U$ es de la forma $\left(-\varepsilon_{1}, \varepsilon_{1}\right) \times \cdots \times\left(-\varepsilon_{n}, \varepsilon_{n}\right)$ para ciertos números positivos $\varepsilon_{1}, \ldots, \varepsilon_{n}$.
} 
Observación 4.14. Un caso que nos interesa particularmente es el de subactuación 1. En tal caso, el paso 4 se cumple inmediatamente puesto que $\mu=\nu=1$. Además, como $\mathbb{M}=\lambda_{\text {mín }}^{\mathbb{M}}=\partial f^{1} / \partial q^{1}(\mathbf{0})$, el paso 6 consiste en verificar que $\partial f^{1} / \partial q^{1}(\mathbf{0})>0$ y el paso 7 , a su vez, se reduce a escoger $\kappa$ cumpliendo

$$
\kappa>\frac{\sum_{b=1}^{n-1}\left(\partial f^{1} / \partial q^{1+b}(\mathbf{0})\right)^{2}}{\partial f^{1} / \partial q^{1}(\mathbf{0})} .
$$

El procedimiento anterior permite encontrar soluciones de las ecuaciones potenciales siempre que seamos capaces de hallar una solución $\delta$ de las ecuaciones (4.31) que además cumpla (4.34). En la sección siguiente, utilizando el contexto provisto por el Lema 4.4 y el Teorema 4.1, veremos que para los sistemas 1-subactuados esto siempre puede hacerse. Más aun, veremos que $\delta$ también puede construirse por cuadraturas $y$, por lo tanto, daremos un conjunto de instrucciones completo para hallar soluciones locales de las ecuaciones cinéticas y potenciales, ambas computables por cuadraturas.

\subsection{Soluciones por cuadraturas de las ecuaciones cinéticas para sistemas 1- subactuados}

Supongamos ahora que el sistema $(H, \mathcal{W})$ tiene grado de subactuación 1, i.e. $\mathcal{W}$ está dado por un subfibrado $W \subset T^{*} Q$ de rango $m=n-1$. Para simplificar notación, será conveniente utilizar la siguiente escritura para las coordenadas del Lema 4.4:

$$
q^{1}=x, \quad q^{1+a}=y^{a}, \quad a=1, \ldots, n-1 .
$$

Escribiremos también

$$
\gamma_{a}^{1}=\gamma_{a}, \quad \delta^{11}=\delta, \quad \bar{p}_{1}^{k}=\bar{p}^{k}, \quad A^{1 k}=A^{k}, \quad \text { y } \quad B_{1}^{11}=B .
$$

Supongamos ahora que ya efectuamos los pasos 1 y 2 del procedimiento para construir una solución de las ecuaciones potenciales descripto en la sección anterior y continuemos con el paso 3 . En el caso 1-subactuado, (4.31) se reduce a una única ecuación,

$$
\frac{\partial \delta}{\partial x}=B \delta
$$

que podemos integrar de la siguiente manera:

$$
\delta(x, \vec{y})=P(\vec{y}) e^{\int_{0}^{x} B(t, \vec{y}) \mathrm{d} t},
$$

donde $P$ es alguna función positiva. Por otro lado, como anticipamos en la Observación 4.14, el paso 4 se cumple automáticamente. 
4. Soluciones por cuadraturas de las ecuaciones cinéticas y potenciales para sistemas 1-subactuados

Luego, siguiendo el paso 5 y dado que $f^{\mu}=f^{1}=\delta\left(\bar{p}^{1} \partial h / \partial x+\bar{p}^{1+a} \partial h / \partial y^{a}\right)$, podemos tomar

$$
v=P(\vec{y}) \int_{0}^{x} e^{\int_{0}^{t} B(r, \vec{y}) \mathrm{d} r}\left(\bar{p}^{1} \frac{\partial h}{\partial x}+\bar{p}^{1+a} \frac{\partial h}{\partial y^{a}}\right)(t, \vec{y}) \mathrm{d} t+\frac{\kappa}{2} \sum_{a=1}^{n-1}\left(y^{a}\right)^{2} .
$$

donde $\kappa>0$. Ahora, siguiendo el paso 6 (ver Observación 4.14), tenemos que verificar que

$$
\frac{\partial^{2} v}{\partial x^{2}}(\mathbf{0})=\frac{\partial f^{1}}{\partial x}(\mathbf{0})>0
$$

que en este caso resulta

$$
P_{0}\left(\bar{p}^{1} \frac{\partial^{2} h}{\partial x^{2}}+\bar{p}^{1+a} \frac{\partial^{2} h}{\partial x \partial y^{a}}\right)(\mathbf{0})>0
$$

donde $0<P_{0}=P(0, \ldots, 0)$.

Por último, según el paso 7 , tenemos que escoger la constante $\kappa$ tal que

$$
\kappa>P_{0}\left[\frac{\sum_{b=1}^{m}\left(\bar{p}^{1} \frac{\partial^{2} h}{\partial x \partial y^{b}}+\bar{p}^{1+a} \frac{\partial^{2} h}{\partial y^{a} \partial y^{b}}\right)^{2}}{\left(\bar{p}^{1} \frac{\partial^{2} h}{\partial x^{2}}+\bar{p}^{1+a} \frac{\partial^{2} h}{\partial x \partial y^{a}}\right)}\right](\mathbf{0}) .
$$

Antes de enunciar el teorema de construcción de soluciones por cuadraturas para sistemas con grado de subactuación 1, estudiaremos la condición (4.45).

\subsubsection{Positividad de las soluciones por cuadraturas para sistemas 1-subactuados}

Para analizar con más cuidado la condición impuesta sobre el sistema por (4.45), debemos calcular los números $\bar{p}^{k}(\mathbf{0})$. Para ello, tenemos que encontrar la matriz de cambio de marcos $\bar{p}(\mathbf{0})$ [ver (4.12)]. Los números $\bar{p}^{k}(\mathbf{0})$ están dados en tal caso por la primera columna de dicha matriz.

Para comenzar, usando el Lema 4.4 tenemos que las coordenadas $(x, \vec{y})$ fijadas cumplen

$$
W_{\bar{q}}=\operatorname{span}\left\{\mathrm{d} y^{a}\right\}_{a=1}^{n-1} \text {. }
$$

Luego, es claro que

$$
W_{\bar{q}}^{\sharp}=\operatorname{span}\left\{\rho^{\sharp}\left(\mathrm{d} y^{a}\right)\right\}_{a=1}^{n-1}
$$

o bien usando las entradas de la representación matricial de $\rho$

$$
W_{\bar{q}}^{\sharp}=\operatorname{span}\left\{\mathbb{H}(\bar{q})^{1,1+a} \frac{\partial}{\partial x}+\mathbb{H}(\bar{q})^{1+a, 1+b} \frac{\partial}{\partial y^{a}}\right\}_{a=1}^{n-1} .
$$

Por lo tanto, el espacio (4.26) está dado en este caso por

$$
A=\operatorname{span}\left\{\frac{\partial}{\partial x}\right\} .
$$

Como complemento $V^{\sharp}$ tomaremos

$$
V^{\sharp}=\operatorname{span}\left\{X \frac{\partial}{\partial x}+Y^{a} \frac{\partial}{\partial y^{a}}\right\},
$$


donde $(X, \vec{Y})$ es un vector de funciones. Definiendo una matriz $\mathbb{J}^{4}$ como

$$
\mathbb{J}^{11}=X, \quad \mathbb{J}^{1+a, 1}=Y^{a}, \quad \mathrm{y} \quad \mathbb{J}^{j, 1+b}=\mathbb{H}^{j, 1+b},
$$

la condición $V^{\sharp} \cap W^{\sharp}=\{0\}$ se cumple si y sólo si $\operatorname{det} \mathbb{J}(\bar{q}) \neq 0$. Para continuar necesitamos calcular el generador $E^{1}$ de $V$ en $\bar{q}$ siguiendo la Ecuación (4.28). Un cálculo sencillo muestra que

$$
p_{V^{\sharp}}\left(\left.\frac{\partial}{\partial x}\right|_{\bar{q}}\right)=\mathbb{J}_{11}(\bar{q})\left(\left.X(\bar{q}) \frac{\partial}{\partial x}\right|_{\bar{q}}+\left.Y^{a}(\bar{q}) \frac{\partial}{\partial y^{a}}\right|_{\bar{q}}\right),
$$

con

$$
\mathbb{J}_{11}(\bar{q})=\frac{M_{11}(\mathbb{J}(\bar{q}))}{\operatorname{det} \mathbb{J}(\bar{q})}=\frac{M_{11}(\mathbb{H}(\bar{q}))}{\operatorname{det} \mathbb{J}(\bar{q})},
$$

donde $M_{11}(\mathbb{H}(\bar{q}))$ es el menor del elemento 11 de la matriz $\mathbb{H}(\bar{q})$. Luego,

$$
\begin{aligned}
E^{1}(\bar{q})= & \frac{M_{11}(\mathbb{H}(\bar{q}))}{\operatorname{det} \mathbb{J}(\bar{q})} \rho^{b}\left(X(\bar{q}) \frac{\partial}{\partial x}+Y^{a}(\bar{q}) \frac{\partial}{\partial y^{a}}\right) \\
= & \frac{M_{11}(\mathbb{H}(\bar{q}))}{\operatorname{det} \mathbb{J}(\bar{q})}\left[X(\bar{q})\left(\mathbb{H}_{11}(\bar{q}) \mathrm{d} x+\mathbb{H}_{1,1+b}(\bar{q}) \mathrm{d} y^{b}\right)\right. \\
& \left.+Y^{a}(\bar{q})\left(\mathbb{H}_{1,1+a}(\bar{q}) \mathrm{d} x+\mathbb{H}_{1+a, 1+b}(\bar{q}) \mathrm{d} y^{b}\right)\right] \\
= & \frac{M_{11}(\mathbb{H}(\bar{q}))}{\operatorname{det} \mathbb{J}(\bar{q})}\left[\left(X \mathbb{H}_{11}+\mathbb{H}_{1,1+a} Y^{a}\right)(\bar{q}) \mathrm{d} x\right. \\
& \left.+\left(X \mathbb{H}_{1,1+b}+Y^{a} \mathbb{H}_{1+a, 1+b}\right)(\bar{q}) \mathrm{d} y^{b}\right] .
\end{aligned}
$$

Dado que

$$
\mathbb{H}_{i j}=\frac{(-1)^{i+j} M_{i j}(\mathbb{H})}{\operatorname{det} \mathbb{H}}
$$

y como $M_{i 1}(\mathbb{H})=M_{i 1}(\mathbb{J})$, se tiene que

$$
X \mathbb{H}_{11}+\mathbb{H}_{1,1+a} Y^{a}=\operatorname{det} \mathbb{J},
$$

y por lo tanto

$$
E^{1}(\bar{q})=\frac{M_{11}(\mathbb{H}(\bar{q}))}{\operatorname{det} \mathbb{H}(\bar{q})} \mathrm{d} x+\eta_{a} \mathrm{~d} y^{a}
$$

para algunos números $\eta_{a}$. Dado que $E^{a}(\bar{q}) \in W_{\bar{q}}$ para todo $a$, es claro que podemos escribir la relación entre los marcos adaptados y los marcos coordenados en $\bar{q}$ del siguiente modo:

$$
\left(\begin{array}{c}
E^{1}(\bar{q}) \\
E^{2}(\bar{q}) \\
\vdots \\
E^{n}(\bar{q})
\end{array}\right)=\underbrace{\left(\begin{array}{cccc}
\frac{M_{11}(\mathbb{H}(\bar{q}))}{\operatorname{det} \mathbb{H}(\bar{q})} & \eta_{1} & \ldots & \eta_{n-1} \\
0 & & & \\
\vdots & & T_{n-1} & \\
0 & & &
\end{array}\right)}_{p(\bar{q})}\left(\begin{array}{c}
\mathrm{d} x \\
\mathrm{~d} y^{1} \\
\vdots \\
\mathrm{d} y^{n-1}
\end{array}\right),
$$

\footnotetext{
${ }^{4}$ Esta matriz consiste en la matriz $\mathbb{H}$ donde se reemplaza la primera columna por el vector columna $(X, \vec{Y})^{t}$.
} 
4. Soluciones por cuadraturas de las ecuaciones cinéticas y potenciales para sistemas 1-subactuados

donde $T_{n-1}$ es una matriz invertible de dimensión $n-1$. A partir de $p(\bar{q})$, no es difícil encontrar que la primera columna de $\bar{p}(\bar{q})$ está dada por el vector

$$
\left(\begin{array}{c}
\operatorname{det} \mathbb{H}(\bar{q}) \\
M_{11}(\mathbb{H}(\bar{q})) \\
0 \\
\vdots \\
0
\end{array}\right)
$$

Luego, es claro que $\bar{p}^{1+a}(\mathbf{0})=0$ para todo $a \mathrm{y}$

$$
\bar{p}^{1}(\mathbf{0})=\frac{\operatorname{det} \mathbb{H}(\mathbf{0})}{M_{11}(\mathbb{H}(\mathbf{0}))} .
$$

Finalmente, la condición (4.45) puede escribirse

$$
P_{0}\left(\frac{\operatorname{det} \mathbb{H}}{M_{11}(\mathbb{H})} \frac{\partial^{2} h}{\partial x^{2}}\right)(\mathbf{0})>0 .
$$

Dado que $\mathbb{H}$ es definida positiva, $M_{11}(\mathbb{H})=\operatorname{det} \mathbb{H}_{\underline{n-1}}>0$. Luego, como $P_{0}, \operatorname{det} \mathbb{H}>0$, la condición de positividad se escribe

$$
\frac{\partial^{2} h}{\partial x^{2}}(\mathbf{0})>0
$$

Por otro lado, la desigualdad (4.46) se reduce a

$$
\kappa>P_{0}\left[\frac{\operatorname{det} \mathbb{H}}{M_{11}(\mathbb{H})} \frac{\sum_{b=1}^{m}\left(\frac{\partial^{2} h}{\partial x \partial y^{b}}\right)^{2}}{\frac{\partial^{2} h}{\partial x^{2}}}\right](\mathbf{0}) .
$$

La condición (4.48) es válida en cualquier carta coordenada como las del Lema 4.4. En particular, las cartas adaptadas a $W$ para sistemas regularmente actuados que introdujimos en el Capítulo 3 son de este tipo. En dicho capítulo vimos que un sistema 1-subactuado cuya linealización en $\bar{\alpha}$ es no controlable cumpliendo (4.48) es tipo 2 (recordar Definición 3.2) y que esto es una condición suficiente para que las soluciones sean definidas positivas (ver Teorema 3.1 junto con la Observación que le sigue). Tenemos entonces el siguiente procedimiento para construir una solución local definida positiva del conjunto de ecuaciones cinéticas y potenciales

Teorema 4.3 (Construcción de soluciones por cuadraturas). Sea $(H, \mathcal{W})$ un sistema Hamiltoniano con grado de subactuación 1 . Sea $\bar{\alpha} \in T_{\bar{q}}^{*} Q$ un punto de equilibrio inestable de $X_{H}$ y supongamos que el sistema es tipo 2. Luego, si

1. fijamos coordenadas $(U,(x, \vec{y}))$ centradas en $\bar{q}$ tales que

$$
W_{\bar{q}}=\operatorname{span}\left\{\left.\mathrm{d} y^{a}\right|_{\bar{q}}\right\}_{a=1}^{n-1},
$$

2. fijamos un complemento local $V$ de $W$ (o bien un complemento $V^{\sharp}$ de $W^{\sharp}$ ), 
3. definimos $E^{1}=\rho^{b}\left(p_{V^{\sharp}}(\partial / \partial x)\right)$ [ver (4.28)], un marco $E^{1+a}$ de $W$, $\bar{p}$ como en (4.12), A y $B$ como en (4.18), (4.19) y (4.42) [ver (4.17)],

4. fijamos una función positiva $P$,

5. tomamos $\gamma$ definida como en (4.29) y (4.30) y $\delta$ definida como en (4.43),

6. fijamos una constante $\kappa$ cumpliendo (4.49) y

7. tomamos $v$ definida como en (4.44);

las funciones, $\delta, \gamma y v$ resuelven las ecuaciones (4.20) y (4.21).

Vale la pena destacar que el teorema anterior construye las funciones $\delta, \gamma$ y $v$ a menos de cuadraturas en coordenadas arbitrarias como las del Lema 4.4. En el capítulo siguiente, a diferencia, hallaremos soluciones en coordenadas específicas que no son computables (necesariamente) por cuadraturas.

\subsubsection{Coordenadas que rectifican $W$}

Supongamos ahora que

$$
W_{q}=\operatorname{span}\left\{\left.\mathrm{d} y^{a}\right|_{q}\right\}_{a=1}^{n-1}, \quad \forall q \in U,
$$

y busquemos el vector $(X, \vec{Y})$ para el cual $V=\operatorname{span}\{\mathrm{d} x\}$. Podemos repetir los cómputos de la sección anterior (esta vez válidos para todo $q \in U$ ) para encontrar [recordar (4.47)]

$$
E^{1}=\frac{M_{11}(\mathbb{H})}{\operatorname{det} \mathbb{J}}\left[\left(X \mathbb{H}_{11}+\mathbb{H}_{1,1+a} Y^{a}\right) \mathrm{d} x+\left(X \mathbb{H}_{1,1+b}+Y^{a} \mathbb{H}_{1+a, 1+b}\right) \mathrm{d} y^{b}\right],
$$

de modo que escogiendo

$$
Y^{a}:=-X\left(\mathbb{H}_{\underline{n-1}}^{-1}\right)^{1+a, 1+b} \mathbb{H}_{1,1+b}
$$

queda

$$
E^{1}=\frac{M_{11}(\mathbb{H})}{\operatorname{det} \mathbb{H}} \mathrm{d} x
$$

con lo cual $V=\operatorname{span}\{\mathrm{d} x\}$. Veamos ahora qué expresión adopta el morfismo $\gamma$ definido por (4.29) y (4.30). Usando los marcos coordenados

$$
\begin{aligned}
\gamma\left(E^{1}\right) & =-\left(\rho^{b} \circ \tilde{\gamma} \circ \rho^{\sharp}\right)\left(\rho^{b} \circ p_{V^{\sharp}}\left(\frac{\partial}{\partial x}\right)\right)=-\rho^{b}\left(\frac{\partial}{\partial x}\right)+E^{1} \\
& =-\mathbb{H}_{11} \mathrm{~d} x-\mathbb{H}_{1,1+a} \mathrm{~d} y^{a}+\frac{M_{11}(\mathbb{H})}{\operatorname{det} \mathbb{H}} \mathrm{d} x,
\end{aligned}
$$

y como

$$
\mathbb{H}_{11}=\frac{M_{11}(\mathbb{H})}{\operatorname{det} \mathbb{H}}
$$

obtenemos

$$
\gamma\left(E^{1}\right)=-\mathbb{H}_{1,1+a} E^{1+a} .
$$


4. Soluciones por cuadraturas de las ecuaciones cinéticas y potenciales para sistemas 1-subactuados

En otras palabras, las componentes $\gamma_{a}$ que dan la representación matricial del morfismo $\gamma$ respecto de los marcos adpatados son $\gamma_{a}=-\mathbb{H}_{1,1+a}$. Sin embargo, para poder comparar con las expresiones para sistemas regularmente actuados, necesitamos las constantes $\gamma_{a}$ relacionadas con la expresión de $\gamma$ respecto de los marcos coordenados. Computando

$$
\begin{aligned}
\gamma(\mathrm{d} x) & =\frac{\operatorname{det} \mathbb{H}}{M_{11}(\mathbb{H})} \gamma\left(\frac{M_{11}(\mathbb{H})}{\operatorname{det} \mathbb{H}} \mathrm{d} x\right)=\frac{\operatorname{det} \mathbb{H}}{M_{11}(\mathbb{H})} \gamma\left(E^{1}\right) \\
& =-\frac{\operatorname{det} \mathbb{H}}{M_{11}(\mathbb{H})} \mathbb{H}_{1,1+a} E^{1+a}=-\frac{\operatorname{det} \mathbb{H}}{M_{11}(\mathbb{H})} \mathbb{H}_{1,1+a} \mathrm{~d} y^{a},
\end{aligned}
$$

por lo cual, respecto de los marcos coordenados,

$$
\gamma_{a}=-\frac{\operatorname{det} \mathbb{H}}{M_{11}(\mathbb{H})} \mathbb{H}_{1,1+a} .
$$

Esta última expresión refleja, en el caso de sistemas regularmente actuados, por qué la construcción de la sección anterior es útil. En efecto, recordando la expresión del vector $A^{k}$ para estos sistemas [ver (3.19)] y usando (4.50), vemos que

$$
A^{k}=\mathbb{H}^{1 k}+\mathbb{H}^{k, 1+a} \frac{\operatorname{det} \mathbb{H}}{M_{11}(\mathbb{H})} \mathbb{H}_{1,1+a}=\mathbb{H}^{1 k}+\left(\delta_{1}^{k}-\mathbb{H}^{k, 1+a} \mathbb{H}_{11}\right) \frac{\operatorname{det} \mathbb{H}}{M_{11}(\mathbb{H})}=\delta_{1}^{k} \frac{\operatorname{det} \mathbb{H}}{M_{11}(\mathbb{H})},
$$

de modo que la ecuación cinética (3.20) se escribe

$$
\frac{\operatorname{det} \mathbb{H}}{M_{11}(\mathbb{H})} \frac{\partial \delta}{\partial q^{1}}-B \delta=0,
$$

o bien

$$
\frac{\partial \delta}{\partial q^{1}}=\frac{M_{11}(\mathbb{H})}{\operatorname{det} \mathbb{H}} B \delta
$$

mientras que las ecuaciones potenciales (3.21)

$$
\frac{\operatorname{det} \mathbb{H}}{M_{11}(\mathbb{H})} \frac{\partial v}{\partial q^{1}}-\frac{\partial h}{\partial q^{1}} \delta=0
$$

o bien

$$
\frac{\partial v}{\partial q^{1}}=\frac{M_{11}(\mathbb{H})}{\operatorname{det} \mathbb{H}} \frac{\partial h}{\partial q^{1}} \delta
$$

Vale la pena enfatizar que el formalismo desarrollado en este capítulo difiere sustancialmente del caso regularmente actuado. Las soluciones que se construyen con el Teorema 4.3 son computables a partir de coordenadas locales arbitrarias alrededor de $\bar{q}$, realizando manipulaciones estrictamente algebraicas con la excepción de las integrales primeras de (4.43) y (4.44). En el caso regularmente actuado, si bien podrían haberse escogido las constantes $\gamma$ como en (4.50) y simplificar las ecuaciones (3.20) y (3.21) como acabamos de mostrar, aun faltaría dar una prescripción de cómo encontrar las coordenadas adaptadas a $W$. Dicho problema constituye un sistema de ecuaciones diferenciales ordinarias y si bien es sabido que dicho sistema tiene solución, podría no ser computable por cuadraturas.

Por último, el análisis que hemos presentado muestra ser satisfactorio para sistemas subactuados tipo 2, dejando fuera los de tipo 1. En el capítulo que viene desarrollaremos otro formalismo para hallar 
soluciones explícitas de sistemas regularmente 1-subactuados que presentan una simetría ante la acción de un grupo de Lie. Dicho formalismo se podrá aplicar también a sistemas tipo 1, como el ejemplo el péndulo doble invertido tratado al final del Capítulo siguiente. 



\section{SOLUCIONES EXPLÍ́CITAS PARA SISTEMAS 1-SUBACTUADOS CON SIMETRÍA}

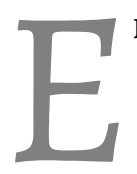

L objetivo de este capítulo es presentar un procedimiento alternativo de construcción explícita de soluciones para las ecuaciones cinéticas y potenciales. En el capítulo anterior presentamos dos procedimientos para construir soluciones explícitas de dichas ecuaciones, que además son computables por cuadraturas. El primero construye una solución de las ecuaciones potenciales para sistemas con grado de subactuación arbitrario a partir de una solución de las ecuaciones cinéticas que satisfacen una condición de integrabilidad. El segundo completa dicho procedimiento, construyendo una solución de las ecuaciones cinéticas para el caso 1-subactuado. También probamos, en este último caso, que la condición necesaria y suficiente para que tales soluciones sean definidas positivas, es que el sistema sea tipo 2.

En este capítulo desarrollaremos un procedimiento que es también aplicable al caso de sistemas tipo 1. Para ello, supondremos que los sistemas considerados presentan una simetría frente a la acción de un grupo de Lie $G$ que precisaremos más adelante.

El capítulo está organizado de la siguiente manera. En primer lugar, suponiendo que contamos con una solución de las ecuaciones cinéticas, trabajaremos con las ecuaciones potenciales para un sistema subactuado arbitrario. Suponiendo que cierto subfibrado vectorial de $T^{*} Q$ es integrable, encontraremos condiciones de integrabilidad que aseguran la existencia de soluciones locales de estas ecuaciones en las coordenadas que rectifican dicho subfibrado. Más aun, estas soluciones podrán ser calculadas computando integrales ordinarias en esas coordenadas. Además, podremos imitar el análisis de la positividad de las soluciones que realizamos en la Sección \$4.2.3. Al final condensamos estos resultados en un procedimiento para construir soluciones.

En segundo lugar, trabajamos con sistemas 1-subactuados que presentan una simetría frente a la acción de un grupo de Lie. Para estos sistemas, demostramos que existen coordenadas en las cuales el término cinético del Hamiltoniano depende de una única coordenada, lo que nos permite hallar solu- 
ciones de las ecuaciones cinéticas con las mismas características, i.e. que dependen únicamente de esa coordenada. Seguidamente, mostramos que es posible combinar estos resultados con el procedimiento mencionado en el párrafo anterior para dar un conjunto completo de instrucciones para construir soluciones explícitas de las ecuaciones cinéticas y potenciales. En particular, mostramos un cambio de coordenadas específico que vincula ambas construcciones y hace posible esta combinación.

Por último, ilustramos estos resultados con un ejemplo, donde puede verse que no es necesario que el sistema sea tipo 2 para que la construcción tenga éxito. De hecho, probaremos que basta que se cumplan las condiciones de positividad enunciadas en los Lemas 3.1 y 3.2 para que las soluciones sean definidas positivas. En ese sentido, estos resultados son aplicables a una familia de sistemas no incluída en los sistemas trabajados en el Capítulo 4.

\subsection{Soluciones explícitas de las ecuaciones potenciales para una clase de sis- temas $m$-subactuados}

A lo largo de esta sección, consideraremos que $(H, \mathcal{W})$ es un sistema subactuado con $H$ simple y $\mathcal{W}$ dado por un subfibrado $W$ de $T^{*} Q$ de rango $m$. Recordemos que, como vimos en el Capítulo 2, las ecuaciones potenciales están dadas intrínsecamente por la ecuación (2.48), i.e.

$$
\langle\mathrm{d} v(\pi(\sigma)), \mathbb{F} \mathfrak{h}(\sigma)\rangle-\langle\mathrm{d} h(\pi(\sigma)), \mathbb{F} \mathfrak{v}(\sigma)\rangle=0, \quad \forall \sigma \in \hat{W}
$$

donde $H=\mathfrak{h}+h \circ \pi, V=\mathfrak{v}+v \circ \pi$ y $\hat{W}=\mathbb{F}^{-1}\left(W^{\circ}\right)$. Recordemos también que los términos cinéticos de $H$ y $V$ están dados por dos métricas Riemannianas, respectivamente $\rho$ y $\phi$, i.e.

$$
\mathfrak{h}(\alpha)=\frac{1}{2}\left\langle\alpha, \rho^{\sharp}(\alpha)\right\rangle \quad \text { y } \quad \mathfrak{v}(\alpha)=\frac{1}{2}\left\langle\alpha, \phi^{\sharp}(\alpha)\right\rangle .
$$

En esta sección supondremos conocida una solución $\mathfrak{v}$ de las ecuaciones cinéticas (2.47), de modo que nuestra única incógnita es $v$.

\subsubsection{Una expresión alternativa de las ecuaciones potenciales}

Dado que los tensores $\rho$ y $\phi$ son simétricos, las ecuaciones potenciales (2.48) pueden escribirse

$$
\langle\alpha, \mathbb{F} \mathfrak{h}[\mathrm{d} v(q)]-\mathbb{F} \mathfrak{v}[\mathrm{d} h(q)]\rangle=0, \quad \forall \alpha \in \hat{W},
$$

i.e.

$$
\mathbb{F} \mathfrak{h}[\mathrm{d} v(q)]-\mathbb{F} \mathfrak{v}[\mathrm{d} h(q)] \in \hat{W}^{\circ},
$$

donde $q=\pi(\alpha)$. Luego, definiendo

$$
\Psi:=\mathbb{F} \mathfrak{h}^{-1} \circ \mathbb{F} \mathfrak{v}
$$


y teniendo en cuenta que $\hat{W}^{\circ}=\mathbb{F} \mathfrak{v}(W)$, se sigue que (5.1) puede reescribirse como

$$
\mathrm{d} v-\Psi \circ \mathrm{d} h \in \Psi(W),
$$

o equivalentemente

$$
\left.(\mathrm{d} v-\Psi \circ \mathrm{d} h)\right|_{\Psi(W)^{\circ}}=0 .
$$

\subsubsection{Condiciones de integrabilidad local}

Continuando con nuestro análisis, supongamos que el subfibrado $\Psi(W)$ es integrable alrededor de $\bar{q} \in Q$. En tal caso, podemos escoger una carta coordenada local $(U, \varphi)$ de $Q$ centrada en $\bar{q}$ tal que $\Psi(W)=\operatorname{span}\left\{\mathrm{d} q^{s+1}, \ldots, \mathrm{d} q^{n}\right\}$ (recordemos que $s=n-m$ ). Al igual que en los capítulos anteriores, identificaremos las funciones $v$ y $h$ con sus representantes en coordenadas locales. Con esta convención y usando las coordendas que introdujimos recién, podemos escribir la Ec. (5.2) como

$$
\frac{\partial v}{\partial q^{\mu}}=\mathbb{H}_{\mu l} \mathbb{V}^{l k} \frac{\partial h}{\partial q^{k}}, \quad \mu \leq s .
$$

De manera similar a lo que presentamos en la Sección \$4.2.2, las condiciones necesarias y suficientes para integrar esta ecuación son

$$
\frac{\partial}{\partial q^{\mu}}\left(\mathbb{H}_{\nu l} \mathbb{V}^{l k} \frac{\partial h}{\partial q^{k}}\right)=\frac{\partial}{\partial q^{\nu}}\left(\mathbb{H}_{\mu l} \mathbb{V}^{l k} \frac{\partial h}{\partial q^{k}}\right),
$$

para todo $\mu, \nu \leq s$, y al igual que antes, en tal caso la solución puede computarse por cuadraturas. Por supuesto, esta condición es un nuevo conjunto de PDEs que debe satisfacer nuestra solución $\mathbb{V}$.

Observación 5.1. Si bien es cierto que las soluciones de (5.3) pueden calcularse por medio de cuadraturas si y sólo si se cumplen las Ecuaciones (5.4), esto no nos permite asegurar que podemos encontrar soluciones de las ecuaciones potenciales por medio de cuadraturas, puesto que primero deberíamos hallar las coordenadas $(U, \varphi)$ mencionadas al comienzo de la sección, que a su vez implica resolver un sistema de ODE. Sin embargo, veremos que para sistemas 1-subactuados con simetría esto siempre puede hacerse.

Vale la pena notar que (5.4) puede expresarse independientemente de coordenadas por la siguiente condición

$$
\left.\mathrm{d}(\Psi \circ \mathrm{d} h)\right|_{\Psi(W)^{\circ}}=0 .
$$

Por otra parte, si fijamos una condición de borde sobre el conjunto

$$
S:=\varphi(U) \cap\left(\{(\underbrace{0, \ldots, 0}_{s})\} \times \mathbb{R}^{m}\right),
$$

sabemos que solo existe un única solución cumpliendo (5.3).

Si $s=1$, i.e. para grado de subactuación 1, el subfibrado $\Psi(W)$ es siempre integrable por razones dimensionales. Además, la condición (5.4) se reduce a una única condición para $\mu=\nu=1$, 
que se cumple inmediatamente. Por lo tanto, si hallamos una solución $\mathfrak{v}$ de las ecuaciones cinéticas, el argumento anterior nos asegura que, para sistemas 1-subactuados, existe una solución para la única ecuación potencial, como es bien sabido (ver Capítulo 3). Más aun, en una carta coordenada apropiada, las soluciones pueden ser calculadas resolviendo integrales primeras.

En cualquier caso, podemos concluir que si $\Psi(W)$ es integrable y se cumple (5.5), entonces (5.2), o equivalentemente las ecuaciones potenciales (2.48), tienen una solución local.

\subsubsection{Condiciones de positividad para las soluciones de las ecuaciones potenciales}

Supongamos ahora que se cumple la Ecuación (5.4) y sea $v$ una solución de (5.3). Entonces, es claro que $v$ es definida positiva alrededor de $\mathbf{0}$ si y sólo si $v$ tiene un mínimo local no degenerado en $\mathbf{0}$ y, como $v$ es una función suave, esto se cumple si y sólo si:

1. 0 es un punto crítico de $v \mathrm{y}$

2. la matriz Hessiana de $v$ en $\mathbf{0}$, es definida positiva.

Fijemos el valor de $v$ sobre el conjunto $S$ [ver Ec. (5.6)] de modo que

$$
v\left(0, \ldots, 0, s^{1}, \ldots, s^{m}\right)=\frac{\kappa}{2} \sum_{a=1}^{m}\left(s^{a}\right)^{2},
$$

con $\kappa>0$ constante, se sigue de (5.3) y de esta condición de borde que $\partial v / \partial v^{i}(\mathbf{0})=0$ para todo $i=1, \ldots, n$. Luego, $\mathbf{0}$ resulta crítico para $v$. Estudiemos ahora la matriz Hessiana de $v$ en $\mathbf{0}$. Notemos que, usando la Ec. (5.3) y el hecho de que $h$ es crítico en $\mathbf{0}$,

$$
\operatorname{Hess}(v)_{\mu \nu}(\mathbf{0})=\left(\mathbb{H}_{\mu l} \mathbb{V}^{l k} \frac{\partial^{2} h}{\partial q^{k} \partial q^{\nu}}\right)(\mathbf{0})=\left(\mathbb{H}_{\mu l} \mathbb{V}^{l k} \operatorname{Hess}(h)_{k \nu}\right)(\mathbf{0}), \quad \forall \mu, \nu \leq s .
$$

Luego, es claro que

$$
\operatorname{Hess}(v)(\mathbf{0})=\left[\begin{array}{cc}
\mathbb{N} & \mathbb{B} \\
\mathbb{B}^{t} & \kappa \mathbb{I}
\end{array}\right],
$$

donde $\mathbb{I}$ es la matriz identidad de $m \times m$, $\mathbb{B}$ es la matriz de $s \times m$ con entradas

$$
\mathbb{B}_{\mu a}:=\left(\mathbb{H}_{\mu l} \mathbb{V}^{l k} \operatorname{Hess}(h)_{k, s+a}\right)(\mathbf{0}), \quad \forall \mu \leq s, a \leq m,
$$

y $\mathbb{N}$ es de $s \times s$ con entradas [recordar (5.8)]

$$
\mathbb{N}_{\mu \nu}:=\left(\mathbb{H}_{\mu l} \mathbb{V}^{l k} \operatorname{Hess}(h)_{k \nu}\right)(\mathbf{0}) .
$$

En esta situación, tenemos la siguiente proposición

Proposición 5.1. Dada una solución local $v$ de (5.3) cumpliendo la condición de borde (5.7), supongamos que la matriz $\mathbb{N}$, dada por (5.8), es definida positiva. Entonces, existe una constante $\kappa$ tal que Hess $(v)(\mathbf{0})$ es definida positiva. 
La demostración es la misma que escribimos para la Proposición 4.4 cambiando $\mathbb{M}$ por $\mathbb{N}$ y $\mathbb{A}$ por $\mathbb{B}$, y puede verse que en este caso basta tomar $\kappa$ tal que

$$
\kappa>\left(\frac{\|\mathbb{B}\|}{\lambda_{\text {mín }}^{\mathbb{N}}}\right)^{2}
$$

donde $\lambda_{\text {mín }}^{\mathbb{N}}$ es el mínimo autovalor de la matriz $\mathbb{B}$.

Dado que $\bar{q}$ es un punto crítico del término potencial $h$, entonces podemos encontrar una expresión intrínseca para la matriz $\mathbb{N}$. Esto se debe a que, en estas condiciones, el tensor Hessiano covariante de $h$, dado por

$$
\operatorname{Hess}(h)=\nabla \nabla h,
$$

siendo $\nabla$ la conexion de Levi-Civita asociada a la métrica Riemanniana $\rho$, tiene por representación matricial en $\bar{q}$ a la matriz Hessiana de $h$. Más específicamente, $\operatorname{Hess}(h) \in \Gamma\left(T^{*} Q \otimes T^{*} Q\right)$ y

$$
\operatorname{Hess}(h)(X, Y)(q)=X(Y h)(q)-\left\langle\mathrm{d} h,\left(\nabla_{X} Y\right)\right\rangle(q),
$$

para todo $X, Y \in \mathfrak{X}(Q)$. Luego, dado que $\bar{q}$ es crítico para $h$, el segundo término se anula y

$$
\operatorname{Hess}(h)(X, Y)(\bar{q})=X^{i}(\bar{q}) \frac{\partial^{2} h}{\partial x^{i} \partial x^{j}}(\bar{q}) Y^{j}(\bar{q}),
$$

que es la expresión conocida de la matriz Hessiana de una función. De todo esto, es fácil ver que $\mathbb{N}$ es la representación local en $\bar{q}$ de la forma bilineal

$$
\mathfrak{N}=\left.\operatorname{Hess}(h) \circ\left(\mathbb{F} \mathfrak{v} \circ \mathbb{F} \mathfrak{h}^{-1} \times \operatorname{id}_{T Q}\right)\right|_{\Psi(W)^{\circ}} .
$$

Para terminar este sección notemos que, si $\mathfrak{N}$ es definida positiva en $\bar{q}$, y si existe una solución local $v$ de (5.2) alrededor de $\bar{q}$, entonces puede escogerse una condición de borde tal que $v$ es definida positiva alrededor de $\bar{q}$.

\subsubsection{Construcción de soluciones explícitas de las ecuaciones potenciales}

Teniendo en cuenta el argumento de las secciones anteriores, tenemos el siguiente teorema de construcción de soluciones para las ecuaciones potenciales.

Teorema 5.1. Sean $\mathfrak{v}$ una solución de las ecuaciones cinéticas y sea $\Psi=\mathbb{F}^{-1} \circ \mathbb{F} \mathfrak{v}$. Si en un entorno de $\bar{q}$

a. el subfibrado $\Psi(W)$ es integrable $y$

b. $\left.\mathrm{d}(\Psi \circ \mathrm{d} h)\right|_{\Psi(W)^{\circ}}=0$,

entonces existe una solución local $v$ de (2.48). Si además

c. la forma bilineal [ver Ec. (5.11)] $\mathfrak{N}$ es definida positiva en $\bar{q}$, 
entonces existe una solución local $v$ (2.48) que es definida positiva alrededor de $\bar{q}$.

Más aun, el desarrollo de las últimas secciones da origen a un procedimiento concreto para construir soluciones explícitas definidas positivas alrededor de $\bar{q}$ de las ecuaciones potenciales, una vez halladas coordenadas que rectifiquen el subfibrado $\Psi(W)$. Más precisamente, debemos seguir las siguientes instrucciones:

1. comprobar que $\Psi(W)$ es integrable alrededor de $\bar{q}$;

2. hallar coordenadas $\left(q^{1}, \ldots, q^{n}\right)$ centradas en $\bar{q}$ tales que $\Psi(W)=\operatorname{span}\left\{\mathrm{d} q^{s+1}, \ldots, \mathrm{d} q^{n}\right\}$;

3. en dichas coordenadas, definir funciones $u_{\mu}:=\mathbb{H}_{\mu l} \mathbb{V}^{l k} \frac{\partial h}{\partial q^{k}}$, para $\mu=1, \ldots, s$;

4. verificar que alrededor del origen $\frac{\partial u_{\nu}}{\partial q^{\mu}}=\frac{\partial u_{\mu}}{\partial q^{\nu}}$ para todo $\mu, \nu \leq s$ [ver Ec. (5.4)];

5. definir $v$ como

$$
v\left(q^{1}, \ldots, q^{n}\right):=\sum_{\mu=1}^{s} \int_{0}^{q^{\mu}} u_{\mu}\left(0, \ldots, 0, t, q^{\mu+1}, \ldots, q^{n}\right) \mathrm{d} t+\frac{\kappa}{2} \sum_{a=1}^{m}\left(q^{s+a}\right)^{2}
$$

para alguna constante $\kappa$;

6. chequear que la matriz $\mathbb{N}$ [recordar Ec. (5.8)] es definida positiva;

7. escoger $\kappa$ de manera tal que [recordar Eqs. (5.9) y (5.10)]

$$
\kappa>\frac{\sum_{b=1}^{m} \sum_{\mu=1}^{s}\left(\partial u_{\mu} / \partial q^{s+b}(\mathbf{0})\right)^{2}}{\lambda_{\text {mín }}^{\mathbb{N}}} .
$$

Observación 5.2. En el caso de subactuación 1, los pasos 1 y 4 no son necesarios puesto que las condiciones de integrabilidad a las que refieren se cumplen automáticamente. Además, dado que $\mathbb{N}=\lambda_{\text {mín }}^{\mathbb{N}}=$ $\partial u_{1} / \partial q^{1}(\mathbf{0})$, el paso 6 consiste en chequear que $\partial u_{1} / \partial q^{1}(\mathbf{0})>0$ y el paso 7 se reduce a escoger $\kappa$ tal que

$$
\kappa>\frac{\sum_{b=1}^{n-1}\left(\partial u_{1} / \partial q^{1+b}(\mathbf{0})\right)^{2}}{\partial u_{1} / \partial q^{1}(\mathbf{0})} .
$$

Por lo tanto, existe solución definida positiva alrededor de $\mathbf{0}$ si $\partial u_{1} / \partial q^{1}(\mathbf{0})>0$.

Tal y como comentamos al comienzo del Capítulo 4, la idea de estudiar las ecuaciones potenciales suponiendo que tenemos una solución de las ecuaciones cinéticas aparece también en la Referencia [30], donde dicha idea se lleva a cabo para las condiciones de matching del energy shaping. En la misma, el autor encuentra condiciones de integrabilidad para las condiciones de matching potenciales usando la teoría de integrabilidad de Goldschmidt (ver [17]). Luego, si estas condiciones se cumplen y si los objetos involucrados son de clase $C^{\omega}$, se concluye que existe solución de las ecuaciones. Sin embargo, no se estudia la positividad de las mismas. En nuestro caso, en cambio, si bien las condiciones de integrabilidad son muy similares a las de [30], el análisis es válido en la categoría $C^{\infty}$. Más aun, damos condiciones necesarias y suficientes para que la solución sea definida positiva y, además, mostramos como construir la solución calculando integrales ordinarias en un sistema de coordenadas apropiado. 


\subsection{Sistemas 1-subactuados con simetría}

En esta sección nos concetraremos en sistemas con grado de subactuación 1 que son "invariantes" frente a la acción de un grupo de Lie $G$ de dimensión $n$ - 1 (en un sentido que será precisado más adelante). Vamos a probar que, bajo ciertas hipótesis, es posible encontrar coordenadas para $Q$ tales que la expresión coordenada del término cinético de la función Hamiltonianana $H$ depende únicamente de una de las coordenadas. Esto nos permitirá construir soluciones explícitas de las ecuaciones cinéticas $y$, junto con el procedimiento para construir soluciones de las ecuaciones potenciales presentado en la sección anterior, tendremos un conjunto de instrucciones completo para construir soluciones explícitas de ambas ecuaciones.

Dado que la simetría cumplirá un rol fundamental en lo que resta del capítulo, haremos ahora una breve introducción de algunas herramientas y estableceremos la notación que usaremos en las siguientes secciones.

\subsubsection{Acciones de Grupos de Lie e invariancia}

Si $G$ es un grupo de Lie actuando sobre una variedad suave $Q$, denotaremos por $\Theta: G \times Q \rightarrow Q$ a la aplicación acción y escribiremos $\Theta(g, q)=g \cdot q$ para simplificar la notación. Si $g \in G$ y $q \in Q$, definamos además las siguientes aplicaciones asociadas a $\Theta$ :

$$
\begin{aligned}
& \Theta^{q}: G \rightarrow Q / \Theta^{q}(g)=g \cdot q, \\
& \Theta_{g}: Q \rightarrow Q / \Theta_{g}(q)=g \cdot q, \\
& \tilde{\Theta}: G \times Q \rightarrow Q \times Q / \tilde{\Theta}(g, q)=(q, g \cdot q) .
\end{aligned}
$$

Diremos que $\Theta$ es libre si $\Theta^{q}$ es inyectiva para todo $q \in Q$ y que es propia si $\tilde{\Theta}$ es una aplicación propia (en el sentido de que preimágenes de conjuntos compactos son compactos). Si $q \in Q$, la órbita de $q$ es el conjunto $G \cdot q:=\{g \cdot q \in Q: g \in G\}$. Es posible mostrar que, si $\Theta$ es libre y propia, entonces $G \cdot q$ es una subvariedad regular de $Q$ (con $\Theta^{q}$ un embedding) para todo $q \in Q$.

Denotaremos por $L_{g}: G \rightarrow G$ a la traslación a izquierda por un elemento $g \in G$, i.e. $L_{g}(h)=g \cdot h$. Diremos que un campo vectorial $X \in \mathfrak{X}(G)$ es invariante a izquierda si $L_{g *}(X(h))=X(g \cdot h)$, para todo $g, h \in G$. El conjunto de todos los campos ivariantes a izquierda será denotado $\operatorname{Lie}(G) \subset \mathfrak{X}(G)$. Consideremos ahora $\eta \in \operatorname{Lie}(G)$ no nulo. El generador infinitesimal (o campo vectorial fundamental) de $\eta$ se define como

$$
\eta_{Q}(q):=\Theta_{* e}^{q}(\eta(e))
$$

donde $e \in G$ es el elemento neutro del grupo. Observemos que, si $\left\{\eta_{i}(e)\right\}$ es una base de $T_{e} G$, entonces $\left\{\eta_{i} Q(q)\right\}$ es una base de $T_{q}(G \cdot q)$.

Dados $X \in \mathfrak{X}(Q)$ y $\omega \in \Omega^{\bullet}(Q)$, diremos que son $G$-invariantes si

$$
\Theta_{g *}(X(q))=X(g \cdot q) \quad \text { у } \quad \Theta_{g^{-1}}^{*}(\omega(q))=\omega(g \cdot q),
$$


para todo $g \in G$ y $q \in Q$. Dada una 2-forma $\rho$, diremos que su forma cuadrática asociada es $G$ invariante si sólo si $\rho$ lo es.

Una distribución $\Delta$ (resp. codistribution $\Sigma$ ) es $G$-invariante si para cada campo vectorial $X \subset \Delta$ (resp. cada forma diferencial $\omega \subset \Sigma$ ) tenemos $\Theta_{g *} X \subset \Delta$ (resp. $\Theta_{g^{-1}}^{*} \omega \subset \Sigma$ ) para todo $g \in G$.

Supongamos ahora que $G$ es un grupo abeliano y consideremos una curva integral $\gamma^{\eta}$ de $\eta$ pasando por $e$ en $t=0$. Entonces,

$$
\gamma_{q}^{\eta_{Q}}=\Theta^{q} \circ \gamma^{\eta}
$$

es una curva integral de $\eta_{Q}$. En efecto, tenemos

$$
\gamma_{q}^{\eta_{Q}}(0)=\Theta^{q}\left(\gamma^{\eta}(0)\right)=\Theta^{q}(e)=e \cdot q=q
$$

y usando la invariancia a izquierda de $\eta$

$$
\dot{\gamma}_{q}^{\eta_{Q}}(t)=\Theta_{* \gamma^{\eta}(t)}^{q}\left(\eta\left(\gamma^{\eta}(t)\right)\right)=\Theta_{* \gamma^{\eta}(t)}^{q}\left(L_{\gamma^{\eta}(t) * e}(\eta(e))\right)=\left(\Theta^{q} \circ L_{\gamma^{\eta}(t)}\right)_{* e}(\eta(e)) .
$$

Pero, como $G$ es abeliano, para todo $g, h \in G$ y $q \in Q$

$$
\Theta^{q} \circ L_{g}(h)=\Theta^{q}(g h)=g h \cdot q=h g \cdot q=h \cdot(g \cdot q)=\Theta^{g \cdot q}(h),
$$

de manera que

$$
\Theta^{q} \circ L_{\gamma^{\eta}(t)}=\Theta^{\gamma^{\eta}(t) \cdot q} .
$$

Insertando esto en (5.14) obtenemos

$$
\dot{\gamma}_{q}^{\eta_{Q}}(t)=\Theta_{* e}^{\gamma^{\eta}(t) \cdot q}(\eta(e))=\eta_{Q}\left(\gamma_{q}^{\eta_{Q}}(t)\right)
$$

lo que confirma que $\gamma_{q}^{\eta_{Q}}=\Theta^{q} \circ \gamma^{\eta}$ es una curva integral de $\eta_{Q}$ padando por $q$.

\subsubsection{Cartas coordenadas $G$-adaptadas}

El objetivo de esta sección es encontrar un sistema de coordenadas conveniente para construir una solución de las ecuaciones cinéticas (2.47). Para ello, usaremos el hecho de que, si la acción es libre y propia, entonces podemos escoger una subvariedad $S \subset Q$, transversal a cada órbita, tal que el espacio de configuraciones es localmente difeomorfo, via $\Theta$, a una variedad producto. Enunciamos con precisión esta afirmación en el siguiente teorema.

Teorema 5.2 (Estructura local de producto). Sean $Q$ una variedad de dimensión $(m+k)$ y $G$ un grupo de Lie de dimensión $k$ que actúa de manera libre y propia sobre $Q$. Entonces, para cada $p \in Q$ existe una subvariedad $S \subset Q$ de deimensión $m$ que contiene a p tal que la restricción de la acción $\chi=\left.\Theta\right|_{G \times S}$ es un difeomorfismo local alrededor de $(e, p)$.

Para una demostración de este resultado puede consultarse, por ejemplo, la Referencia [29]. Este teorema nos provee una poderosa herramienta para construir campos vecotriales y formas diferenciales $G$-invariantes. El siguiente lema muestra cómo esta idea puede usarse para describir codistribuciones $G$-invariantes. 
Lema 5.1. Sean $Q$ y $G$ como en el Teorema 5.2 y sea $W$ una codistribución regular de rango $k$ sobre $Q$ que es $G$-invariante. Entonces, para cada $p \in Q$ existe un entorno abierto $U$ de $p$ y formas diferenciales $G$-invariantes $\xi_{i} \in \Omega^{1}(U), i=1, \ldots, k$, tales que $W_{q}=\operatorname{span}\left\{\xi_{i}(q)\right\}_{i=1}^{k}$ para todo $q \in U$.

Demostración. Dado que $W$ es regular, existe un entorno abierto $U$ de $p$ tal que $W_{q}=\operatorname{span}\left\{\omega_{i}(q)\right\}_{i=1}^{k}$ para alguna familia $\left\{\omega_{i}\right\}_{i=1}^{k}$ de formas diferenciales y para todo $q \in U$. Achicando $U$ (de ser necesario), el Teorema 5.2 dice que existe una subvariedad $S \subseteq U$ de dimensión $m$ tal que $\chi=\left.\Theta\right|_{G \times S}$ es un difeomorfismo sobre $U$. Definamos ahora, para todo $q \in U$,

$$
\xi_{i}(q):=\Theta_{g^{-1}}^{*}\left(\omega_{i}\left(q^{\prime}\right)\right), \quad \forall i=1, \ldots, k,
$$

donde $\left(g, q^{\prime}\right)=\chi^{-1}(q) \in G \times S$. Es sencillo ver que estas aplicaciones definen formas diferenciales suaves no nulas sobre $U$ y que son $G$-invariantes por construcción. Notemos que si $q \in S$, entonces $g=e, q^{\prime}=q$ y $\xi_{i}(q)=\omega_{i}(q)$, por lo que $\left\{\xi_{i}(q)\right\}_{i=1}^{k}$ es un conjunto generador de $W_{q}$ para cada $q \in S$. Más aun, dado que $\Theta_{g^{-1}}^{*}$ es un isomorfismo de espacios vectoriales, aplica conjuntos linealmente independientes en conjuntos linealmente independientes, de manera que el conjunto $\left\{\xi_{i}(q)\right\}_{i=1}^{k}$ genera un subespacio vectorial de $T_{q}^{*} Q$ de dimensión $k$ para todo $q \in U$. Pero, dado que $W$ es $G$-invariante y de dimensión $k$, resulta de hecho que $\operatorname{span}\left\{\xi_{i}(q)\right\}_{i=1}^{k}=W_{q}$ para todo $q \in U$, que era lo que se quería probar.

De ahora en adelante supondremos que el sistema $(H, \mathcal{W})$ tiene función Hamiltoniana simple con término cinético hy y que $\mathcal{W}$ está dado por un subfibrado lineal $W$ de $T^{*} Q$. También supondremos que $G$ es un grupo de Lie abeliano que actúa de manera libre y propia sobre $Q$ vía una acción $\Theta$.

En lo que sigue vamos a necesitar una noción particular de $G$-invariancia para este tipo de sistemas.

Definición 5.1. Diremos que $(H, \mathcal{W})$ es cinético $G$-invariante si la función $\mathfrak{h}$ y la codistribución $W$ son $G$-invariantes.

Notemos que, si la forma cuadrática $\mathfrak{h}$ es $G$-invariante, entonces su métrica Riemanniana asociada $\rho$ es también $G$-invariante, es decir, para todo $q \in Q$ y $g \in G$,

$$
\rho\left(\Theta_{g *} v, \Theta_{g *} w\right)=\rho(v, w), \quad \forall v, w \in T_{q} Q .
$$

Supongamos además que $G$ tiene dimensión $(n-1)$ y que $W$ tiene rango también $n-1$. Este es el tipo de sistemas subactuados que consideraremos de aquí en adelante. Queremos encontrar coordenadas en las cuales $\rho$ dependa únicamente de una coordenada. La siguiente proposición muestra que esto es posible bajo una hipótesis adicional.

Proposición 5.2 (Coordenadas $G$-adaptadas). Sea $G$ un grupo de Lie de dimensión $(n-1)$ actuando de manera libre y propia sobre una variedad suave $Q$ de dimensión $n$, y sea $(H, \mathcal{W})$ un sistema 1-subactuado que es cinético $G$-invariante. Supongamos que la distribución

$$
\Delta_{q}:=W_{q}^{\circ}+T_{q}(G \cdot q), \quad q \in Q,
$$


tiene rango máximo en $\bar{q} \in Q$. Entonces, existe una carta coordenada $\left(U, \varphi=\left(x, y^{1}, \ldots, y^{n-1}\right)\right)$ centrada en $\bar{q}$ tal que, para todo $q \in U, W_{q}=\operatorname{span}\left\{\left.\mathrm{d} y^{a}\right|_{q}\right\}_{a=1}^{n-1} y$ tal que las funciones coordenadas de la métrica $\mathbb{H}_{i j}$, dependen únicamente de la coordenada $x$.

Demostración. Dado que $\Delta$ tiene rango máximo en $\bar{q}$, es localmente un subfibrado vectorial de $T Q$. Más aun, como $\operatorname{dim} W_{q}^{\circ}=1$ para todo $q$, debe suceder que

$$
W_{q}^{\circ} \cap T_{q}(G \cdot q)=\{0\}
$$

en un entorno $V$ de $\bar{q}$. Notemos que la $G$-invariancia de $W$ implica que $W^{\circ}$ es $G$-invariante también. Entonces, imitando la construcción del Lema 5.1, es fácil ver que existe un campo vectorial no nulo y $G$-invariante $\xi^{\prime}$ definido en un abierto $N$ que contiene a $\bar{q}$ tal que $W_{q}^{\circ}=\operatorname{span}\left\{\xi^{\prime}(q)\right\}$ para todo $q \in N$.

Sea $\left\{\eta_{Q a}\right\}_{a=1}^{n-1}$ un sistema de $n-1$ generadores infinitesimales linealmente independientes. Observemos que, dado que $G$ es abeliano, todo generador infinitesimal es $G$-invariante. Como es bien sabido que los campos $G$-invariantes conmutan con todos los generadores infinitesimales, es claro que $\left[\eta_{Q a}, \eta_{Q b}\right]=0$ para todo $a, b=1, \ldots, n-1$. Gracias a la Ec. (5.17), es claro que el conjunto $\left\{\xi^{\prime}, \eta_{Q 1}, \ldots, \eta_{Q n-1}\right\}$ también es linealmente independiente sobre $N \cap V$ y, como $\xi^{\prime}$ es $G$-invariante, tenemos que $\left[\xi^{\prime}, \eta_{Q a}\right]=0$ sobre $N \cap V$ para cada $a=1, \ldots, n-1$. En particular, tenemos un conjunto de $n$ campos vectoriales linealmente independientes que conmutan entre sí. En consecuencia, existe una carta coordenada $(U, \varphi)$ centrada en $\bar{q}$ con $U \subseteq N \cap V$ y tal que

$$
\xi^{\prime}=\frac{\partial}{\partial x} \quad \text { and } \quad \eta_{Q a}=\frac{\partial}{\partial y^{a}}, \quad \forall a=1, \ldots, n-1 .
$$

Finalmente, para todo $q \in U$,

$$
W_{q}=\left(W_{q}^{\circ}\right)^{\circ}=\operatorname{span}\left\{\xi^{\prime}(q)\right\}^{\circ}=\operatorname{span}\left\{\left.\frac{\partial}{\partial x}\right|_{q}\right\}^{\circ}=\operatorname{span}\left\{\left.\mathrm{d} y^{a}\right|_{q}\right\}_{a=1}^{n-1},
$$

como queríamos.

Para completar la demostración, vamos a mostrar que la métrica $\rho$ depende únicamente de la coordenada $x$. Sean $\mathbb{H}_{i j} \in C^{\infty}(U)$ las funciones dadas por

$$
\mathbb{H}_{i j}(q)=\rho\left(\left.\frac{\partial}{\partial q^{i}}\right|_{q},\left.\frac{\partial}{\partial q^{j}}\right|_{q}\right)
$$

donde

$$
\frac{\partial}{\partial q^{1}}=\frac{\partial}{\partial x} \quad \text { y } \quad \frac{\partial}{\partial q^{1+a}}=\frac{\partial}{\partial y^{a}}
$$

Notemos que, gracias a la $G$-invariancia de $\rho$ [ver (5.15)] y la invariancia de los campos vectoriales $\frac{\partial}{\partial x} \mathrm{y} \frac{\partial}{\partial y^{a}}$

$$
\begin{aligned}
\mathbb{H}_{i j}(g \cdot q) & =\rho\left(\left.\frac{\partial}{\partial q^{i}}\right|_{g \cdot q},\left.\frac{\partial}{\partial q^{j}}\right|_{g \cdot q}\right)=\rho\left(\left.\Theta_{g *} \frac{\partial}{\partial q^{i}}\right|_{q},\left.\Theta_{g *} \frac{\partial}{\partial q^{j}}\right|_{q}\right) \\
& =\rho\left(\left.\frac{\partial}{\partial q^{i}}\right|_{q},\left.\frac{\partial}{\partial q^{j}}\right|_{q}\right)=\mathbb{H}_{i j}(q) .
\end{aligned}
$$


Calculemos ahora

$$
\eta_{Q}\left(\mathbb{H}_{i j}\right)(q)=\left.\frac{\mathrm{d}}{\mathrm{d} t}\right|_{t=0} \mathbb{H}_{i j}\left(\gamma_{q}^{\eta_{Q}}(t)\right),
$$

donde $\eta_{Q}$ es un generador infinitesimal y $\gamma_{q}^{\eta_{Q}}(t)$ es una curva integral de $\eta_{Q}$ tal que $\gamma_{q}^{\eta_{Q}}(0)=q$. En el caso en que $\eta_{Q}=\eta_{Q a}$, esta función es la derivada de $\mathbb{H}_{i j}$ respecto de $y^{a}$. Usando la Ec. (5.13) en (5.19), hallamos

$$
\eta_{Q}\left(\mathbb{H}_{i j}\right)(q)=\left.\frac{\mathrm{d}}{\mathrm{d} t}\right|_{t=0} \mathbb{H}_{i j}\left(\Theta^{q}\left(\gamma^{\eta}(t)\right)\right)=\left.\frac{\mathrm{d}}{\mathrm{d} t}\right|_{t=0} \mathbb{H}_{i j}\left(\gamma^{\eta}(t) \cdot q\right)=\left.\frac{\mathrm{d}}{\mathrm{d} t}\right|_{t=0} \mathbb{H}_{i j}(q)=0,
$$

donde hemos usado (5.18).

Dado un sistema 1-subactuado cinético $G$-invariante $(H, \mathcal{W})$ cumpliendo las hipótesis de la Proposición 5.2, diremos que las coordenadas construidas en la demostración anterior son coordenadas $G$-adaptadas. Usando estas coordenadas, el hecho de que las entradas de $\mathbb{H}$ dependen únicamente de la coordenada $x$ será extremadamente útil en la próxima sección, donde encontraremos soluciones explícitas del conjunto de ecuaciones cinéticas y potenciales.

Observación 5.3. Notemos que las coordenadas $G$-adaptadas son también coordenadas adaptadas a $W$ (recordar Definición 3.1). Esto nos permite usar el cambio de variables dependientes mostrado en el Capítulo 3 a las nuevas variables $\delta, \gamma$ y $l$ y por lo tanto podemos optar por resolver las ecuaciones (3.20) en lugar de (2.47) para hallar soluciones de las ecuaciones cinéticas.

\subsection{Soluciones explícitas para sistemas con simetría}

En esta sección, en el contexto de sistemas 1 -subactuados cinético $G$-invariantes, vamos a combinar los resultados de la sección anterior para construir soluciones explícitas de las ecuaciones cinéticas, y el procedimiento descripto en la Sección \$5.1.4 para hallar soluciones explícitas de las ecuaciones potenciales. De aquí en adelante, supondremos que tenemos un sistema subactuado $(H, \mathcal{W})$ cumpliendo las hipótesis de la Proposición 5.2.

\subsubsection{Soluciones de las ecuaciones cinéticas}

Consideremos la única ecuación cinética (3.20) en una carta coordenada $G$-adaptada (ver la Proposición 5.2 y la Observación 5.3) $\left(U, \varphi=\left(x, y^{1}, \ldots, y^{n-1}\right)\right)$. Recordemos que, en estas coordenadas, la codistribución $W$ está generada por las formas diferenciales coordenadas $\mathrm{d} y^{a}$.

Dado que las entradas de la métrica $\mathbb{H}$ no dependen de las coordenadas $y^{a}$ [ver Ec. (5.20)], podemos buscar soluciones $(\delta, \gamma)$ de (3.20) que sólo dependan de la coordenada $x$. Con esto en mente, podemos escribir nuestra ecuación como

$$
\left(\mathbb{H}^{11}-\mathbb{H}^{1,1+b} \gamma_{b}\right) \delta_{x}=B \delta
$$


donde $B=\mathbb{H}_{x}^{11}-2 \mathbb{H}_{x}^{1,1+b} \gamma_{b}+\gamma_{a} \mathbb{H}_{x}^{1+a, 1+b} \gamma_{b}$ [recordar la Ec. (3.18)]. Podemos integrar esta última ecuación en un entorno de $x=0$ para obtener

$$
\delta(x)=P \exp \left(\int_{0}^{x} \frac{B(t)}{\mathbb{H}^{11}(t)-\mathbb{H}^{1,1+b}(t) \gamma_{b}(t)} \mathrm{d} t\right)
$$

donde $P$ es una constante positiva. Claramente, es necesario pedir que $\gamma(0)$ cumpla la condición

$$
\mathbb{H}^{11}(0)-\mathbb{H}^{1,1+b}(0) \gamma_{b}(0) \neq 0
$$

Observación 5.4. Vale la pena decir que siempre es posible fijar el valor de $\gamma$ en $x=0$ para que esta última ecuación se cumpla. En efecto, si $\mathbb{H}^{1,1+b}(0)=0$ para todo $b=1, \ldots, n-1$, la condición se reduce a

$$
\mathbb{H}^{11}(0) \neq 0
$$

que siempre se cumple puesto que $\mathbb{H}$ es definida positiva. Por otro lado, si existe $b_{0}$ tal que $\mathbb{H}^{1,1+b_{0}}(0) \neq$ 0

$$
\gamma_{b_{0}}(0) \neq \frac{\mathbb{H}^{11}(0)-\sum_{\substack{b=1 \\ b \neq b_{0}}}^{n-1} \mathbb{H}^{1,1+b}(0) \gamma_{b}(0)}{\mathbb{H}^{1,1+b_{0}}(0)}
$$

En conclusión, dado un sistema 1-subactuado cinético $G$-invariante $(H, \mathcal{W})$ (cuya distribución $\Delta$ ver Ec. (5.16)- tenga rango máximo en $\bar{q}$ ), para encontrar una solución $\mathbb{V}=\mathbb{V}(x)$ de la ecuación cinética (3.20), podemos proceder de la siguiente manera:

i. encontrar coordenadas $G$-adaptadas $\left(x, y^{1}, \ldots, y^{n-1}\right)$;

ii. fijar $n-1$ funciones $\gamma_{b}(x)$ tales que

$$
\mathbb{H}^{11}(0)-\mathbb{H}^{1,1+b}(0) \gamma_{b}(0) \neq 0
$$

y definir [ver (3.13)]

$$
\begin{gathered}
\mathbb{V}^{11}(x):=\delta(x)+\gamma_{a}(x) \delta^{a b} \gamma_{b}(x), \quad \mathbb{V}^{1,1+a}(x)=\mathbb{V}^{1+a, 1}(x):=\delta^{a b} \gamma_{b}(x) \\
\text { y } \quad \mathbb{V}^{1+a, 1+b}(x):=\delta^{a b},
\end{gathered}
$$

donde $\delta^{a b}$ es el símbolo de Kronecker y $\delta$ está dada por (5.21) para alguna constante positiva $P$. 


\subsubsection{Soluciones de las ecuaciones potenciales}

Ahora, partiendo de las soluciones construidas en la sección anterior, vamos a aplicar el procedimiento que desarrollamos en la Sección \$5.1 para encontrar una solución local $v$ de la ecuación potencial.

Consideremos entonces las coordenadas $G$-adaptadas $\left(x, y^{1}, \ldots, y^{n-1}\right)$ de la Proposición 5.2. Recordemos que, en virtud de la Observación 5.2, no es necesario efectuar los pasos 1 y 4 de nuestro procedimiento. Llevemos a cabo el paso 2. Para comenzar, observemos que

$$
\Psi(W)=\operatorname{span}\left\{\Psi\left(\mathrm{d} y^{a}\right)\right\}_{a=1}^{n-1} .
$$

Escribamos ahora las formas diferenciales $\Psi\left(\mathrm{d} y^{a}\right)$ en términos de las formas diferenciales coordenadas $\mathrm{d} x, \mathrm{~d} y^{b}$, i.e. encontremos funciones $\alpha^{a} \mathrm{y} \beta_{b}^{a}$ tales que

$$
\Psi\left(\mathrm{d} y^{a}\right)=\alpha^{a} \mathrm{~d} x+\beta_{b}^{a} \mathrm{~d} y^{b} .
$$

Calculando,

$$
\begin{aligned}
\alpha^{a} & =\left\langle\Psi\left(\mathrm{d} y^{a}\right), \frac{\partial}{\partial x}\right\rangle=\mathbb{H}_{1 j} \mathbb{V}^{j, 1+a}=\mathbb{H}_{11} \mathbb{V}^{1,1+a}+\mathbb{H}_{1,1+b} \mathbb{V}^{1+b, 1+a} \\
& =\mathbb{H}_{11} \delta^{a b} \gamma_{b}+\mathbb{H}_{1,1+b} \delta^{a b}=\left(\mathbb{H}_{11} \gamma_{b}+\mathbb{H}_{1,1+b}\right) \delta^{a b}
\end{aligned}
$$

y

$$
\begin{aligned}
\beta_{b}^{a} & =\left\langle\Psi\left(\mathrm{d} y^{a}\right), \frac{\partial}{\partial y^{b}}\right\rangle=\mathbb{H}_{1+b, j} \mathbb{V}^{j, 1+a}=\mathbb{H}_{1+b, 1} \mathbb{V}^{1,1+a}+\mathbb{H}_{1+b, 1+c} \mathbb{V}^{1+c, 1+a} \\
& =\mathbb{H}_{11} \delta^{a c} \gamma_{c}+\mathbb{H}_{1,1+b} \delta^{a c}=\left(\mathbb{H}_{1,1+b} \gamma_{c}+\mathbb{H}_{1+c, 1+b}\right) \delta^{a c}
\end{aligned}
$$

Luego, definiendo

$$
\mathbb{Q}_{c b}=\gamma_{c} \mathbb{H}_{1,1+b}+\mathbb{H}_{1+c, 1+b},
$$

tenemos

$$
\Psi\left(\mathrm{d} y^{c}\right)=\delta^{c b}\left(\mathbb{H}_{11} \gamma_{b}+\mathbb{H}_{1,1+b}\right) \mathrm{d} x+\delta^{c b} \mathbb{Q}_{b d} \mathrm{~d} y^{d}
$$

Observación 5.5. Vale la pena notar que podemos escoger los valores de las constantes $\gamma_{b}(\mathbf{0})$ de manera tal que $\mathbb{Q}$ sea una matriz invertible en $\mathbf{0}$. Para mostrar esto, consideremos el determinante de $\mathbb{Q}(\mathbf{0})$ como un polinomio en las variables $\gamma_{b}(\mathbf{0})$. Si $\operatorname{det} \mathbb{Q}(\mathbf{0})$ fuese el polinomio constante, entonces sería no nulo pues, tomando $\gamma_{b}(\mathbf{0})=0$ para todo $b$, obtenemos $\mathbb{Q}(\mathbf{0})_{c b}=\mathbb{H}_{1+c, 1+b}(\mathbf{0})$, que es invertible. Si, por otra parte, $\operatorname{det} \mathbb{Q}(\mathbf{0})$ no es un polinomio constante, entonces existe algún término no nulo

$$
\lambda \gamma_{a_{1}}(\mathbf{0}) \ldots \gamma_{a_{l}}(\mathbf{0})
$$

y podemos tomar $\gamma_{a_{1}}(\mathbf{0}) \ldots \gamma_{a_{l-1}}(\mathbf{0}) \neq 0$ y fijar los números restantes $\gamma_{a}(\mathbf{0})$ con $a \neq a_{l}$ de manera arbitraria. Para esta elección, el determinante resultante $\operatorname{det} \mathbb{Q}(\mathbf{0})$ es un polinomio no constante de 
grado a lo sumo $n-1$ en la variable $\gamma_{a_{l}}(\mathbf{0})$, y por lo tanto existen a lo sumo $n-1$ valores distintos $z_{1}, \ldots, z_{n-1}$ de $\gamma_{a_{l}}(\mathbf{0})$ para los cuales $\operatorname{det} \mathbb{Q}(\mathbf{0})=0$ y es suficiente tomar $\left|\gamma_{a_{l}}(\mathbf{0})\right|>$ máx $\left|z_{a}\right|$. De esta forma, podemos considerar que $\mathbb{Q}$ es invertible alrededor de $\mathbf{0}$.

Supongamos entonces de ahora en adelante que $\mathbb{Q}$ es invertible alrededor de 0 [ver (5.23) y la Observación 5.5]. Bajo esta hipótesis, podemos considerar las formas diferenciales $\xi^{a}$ dadas por

$$
\xi^{a}=\mathbb{Q}^{a b} \delta_{b c} \Psi\left(\mathrm{d} y^{a}\right)=\mathbb{Q}^{a b}\left(\mathbb{H}_{11} \gamma_{b}+\mathbb{H}_{1,1+b}\right) \mathrm{d} x+\mathrm{d} y^{a}
$$

que generan la codistribución $\Psi(W)$. Más aun, son formas diferenciales cerradas y por lo tanto son localmente exactas. De hecho, si definimos las funciones

$$
\varepsilon^{a}(x)=\int_{0}^{x} \mathbb{Q}^{a b}\left(\mathbb{H}_{11} \gamma_{b}+\mathbb{H}_{1,1+b}\right)(t) \mathrm{d} t
$$

y

$$
s^{a}=y^{a}+\varepsilon^{a}(x),
$$

es claro que

$$
\mathrm{d} s^{a}=\xi^{a} .
$$

Dado que el campo vectorial

$$
\frac{\partial}{\partial x}-\mathbb{Q}^{a b}\left(\mathbb{H}_{11} \gamma_{b}+\mathbb{H}_{1,1+b}\right) \frac{\partial}{\partial y^{a}}
$$

genera el anulador $\left(\operatorname{span}\left\{\mathrm{d} s^{a}\right\}_{a=1}^{n-1}\right)^{\circ}$, para completar el paso 2 podemos buscar una forma diferencial exacta $\mathrm{d} r$ tal que

$$
\left\langle\mathrm{d} r, \frac{\partial}{\partial x}-\mathbb{Q}^{a b}\left(\mathbb{H}_{11} \gamma_{b}+\mathbb{H}_{1,1+b}\right) \frac{\partial}{\partial y^{a}}\right\rangle=1
$$

Resolviendo para $r$ obtenemos

$$
r\left(x, y^{1}, \ldots, y^{n-1}\right)=x+\frac{1}{2}\left(y^{a}+\varepsilon^{a}(x)\right) \delta_{a b}\left(y^{b}+\varepsilon^{b}(x)\right) .
$$

Es claro entonces que la asignación $\left(x, y^{1}, \ldots, y^{n-1}\right) \mapsto\left(r, s^{1}, \ldots, s^{n-1}\right)$ es un difeomorfismo local que deja fijo al origen $\mathbf{0}$ y cuya inversa está dada por

$$
\left\{\begin{array}{l}
x=r-\frac{1}{2} s^{a} \delta_{a b} s^{b}, \\
y^{a}=s^{a}-\varepsilon^{a}\left(r-\frac{1}{2} s^{c} \delta_{c d} s^{d}\right) .
\end{array}\right.
$$

Así, terminamos el paso 2.

Observación 5.6. Usando las coordenadas $G$-adaptadas, la ecuación cinética se escribe

$$
\left(\mathbb{H}^{11}-\mathbb{H}^{1,1+a} \gamma_{a}\right) v_{x}+\left(\mathbb{H}^{1,1+b}-\mathbb{H}^{1+b, 1+a} \gamma_{a}\right) v_{y^{b}}=\delta h_{x}
$$

Escribiendo $v(r, \vec{s})=v(x(r, \vec{s}), \vec{y}(r, \vec{s}))$, puede verse que esta ecuación se traduce en

$$
\left(\mathbb{H}^{11}-\mathbb{H}^{1,1+a} \gamma_{a}\right) v_{r}=\delta h_{x}
$$


o equivalentemente [recordar (5.22)]

$$
v_{r}=\frac{\delta h_{x}}{\mathbb{H}^{11}-\mathbb{H}^{1,1+a} \gamma_{a}} .
$$

Esto explica por qué este cambio de coordenadas es útil.

La Ec. (5.27) en la observación anterior dice que la función $u_{1}$ del paso 3 está dada por

$$
u(r, \vec{s}):=u_{1}(r, \vec{s})=\left(\frac{\delta h_{x}}{\mathbb{H}^{11}-\mathbb{H}^{1,1+a} \gamma_{a}}\right)(x(r, \vec{s}), \vec{y}(r, \vec{s})) .
$$

Luego, de acuerdo con el paso 5, una solución de (3.21) está dada por

$$
v(r, \vec{s})=\int_{0}^{r} u(t, \vec{s}) \mathrm{d} t+\kappa \frac{\|\vec{s}\|^{2}}{2}
$$

o bien

$$
v(x, \vec{y})=\int_{0}^{\frac{1}{2}\|\vec{y}-\vec{\varepsilon}(x)\|^{2}+x} u(t, \vec{y}-\vec{\varepsilon}(x)) \mathrm{d} t+\kappa \frac{\|\vec{y}-\vec{\varepsilon}(x)\|^{2}}{2},
$$

utilizando nuestras coordenadas $G$-adaptadas originales. Sólo resta llevar a cabo los pasos 6 y 7 para escoger (de ser posible) la constante $P$, la función $\gamma$ y la constante $\kappa$ de manera tal que (5.28) sea definida positiva. Un cálculo sencillo muestra que

$$
\frac{\partial u}{\partial r}(\mathbf{0})=\frac{\delta(0)}{\left(\mathbb{H}^{11}-\mathbb{H}^{1,1+a} \gamma_{a}\right)^{2}(0)}\left[\left(\mathbb{H}^{11}-\mathbb{H}^{1,1+a} \gamma_{a}\right) h_{x x}+\left(\mathbb{H}^{1,1+b}-\mathbb{H}^{1+b, 1+a} \gamma_{a}\right) h_{x y^{1+b}}\right](\mathbf{0})
$$

Aquí, hemos usado el hecho de que 0 (i.e. $\bar{q}$ ) es un punto crítico para $h$. Luego, el paso 6 se reduce a chequear que

$$
\left[\left(\mathbb{H}^{11}-\mathbb{H}^{1,1+a} \gamma_{a}\right) h_{x x}+\left(\mathbb{H}^{1,1+b}-\mathbb{H}^{1+b, 1+a} \gamma_{a}\right) h_{x y^{1+b}}\right](\mathbf{0})>0
$$

que es una condición en las constantes $\gamma_{a}(\mathbf{0})$.

Observación 5.7. Esta condición fue encontrada ya en el Capítulo 3, donde probamos (ver Lema 3.2)

que existen constantes $\gamma_{a}(\mathbf{0})$ cumpliendo dicha condición si y sólo si la función Hamiltoniana $H$ satisface $h_{x x}(\mathbf{0})>0$ o bien $\left[h_{x x} \mathbb{H}^{1,1+a}+h_{x y^{1+b}} \mathbb{H}^{1+b, 1+a}\right](\mathbf{0}) \neq 0$ para algún $a=1, \ldots, n-1$.

Finalmente, siguiendo el paso 7, dado que (recordar nuevamente que $\mathbf{0}$ es crítico para $h$ )

$$
\frac{\partial u}{\partial s^{b}}(\mathbf{0})=\left[\frac{\delta}{(a-\gamma b)} h_{x y^{1+b}}\right](\mathbf{0})
$$

y que $\delta(\mathbf{0})=P$ [recordar (5.21)], basta tomar [ver Ec. (5.12), (5.29) y (5.31)]

$$
\kappa>P\left[\frac{\sum_{b=1}^{n-1} h_{x y^{1+b}}^{2}}{\left(\mathbb{H}^{11}-\mathbb{H}^{1,1+a} \gamma_{a}\right) h_{x x}+\left(\mathbb{H}^{1,1+b}-\mathbb{H}^{1+b, 1+a} \gamma_{a}\right) h_{x y^{1+b}}}\right](\mathbf{0})
$$

para lograr que la función $v$ construída en (5.28) sea definida positiva en el origen.

Para concluir, enunciamos el siguiente teorema de construcción de soluciones explícitas 
Teorema 5.3 (Construcción de soluciones explícitas). Sea $G$ un grupo de Lie de dimensión $n-1$ que actúa de manera libre y propia sobre una variedad suave $Q$ de dimensión $n$. Sea $(H, \mathcal{W})$ un sistema Hamiltoniano 1-subactuado cinético $G$-invariante sobre $Q$ y consideremos un punto de equilibrio inestable $\bar{\alpha} \in T_{\bar{q}}^{*} Q$ de $X_{H}$. Luego, si $(x, \vec{y})$ son coordenadas $G$-adaptadas para $(H, \mathcal{W})$, y verificamos que

$$
h_{x x}(\mathbf{0})>0 \quad \text { obien } \quad\left[h_{x x} \mathbb{H}^{1,1+a}+h_{x y^{1+b}} \mathbb{H}^{1+b, 1+a}\right](\mathbf{0}) \neq 0 \quad \text { para algún } \quad a=1, \ldots, n-1
$$

podemos construir soluciones locales y definidas positivas de las ecuaciones cinéticas y potenciales del siguiente modo:

1. fijamos $n-1$ funciones $\gamma_{1}(x), \ldots, \gamma_{n-1}(x)$ que cumplan (5.30) y tales que [recordar (5.23) y la Observación 5.5]

$$
\mathbb{H}^{11}(0)-\mathbb{H}^{1,1+b}(0) \gamma_{b}(0) \neq 0 \quad y \quad \operatorname{det} \mathbb{Q}(\mathbf{0}) \neq 0,
$$

$y$ definamos

$$
\begin{gathered}
\mathbb{V}^{11}(x):=\delta(x)+\gamma_{a}(x) \delta^{a b} \gamma_{b}(x), \quad \mathbb{V}^{1,1+a}(x)=\mathbb{V}^{1+a, 1}(x):=\delta^{a b} \gamma_{b}(x) \\
y \quad \mathbb{V}^{1+a, 1+b}(x):=\delta^{a b},
\end{gathered}
$$

donde $\delta^{a b}$ es el símbolo de Kronecker y $\delta$ está dada por (5.21) para alguna constante positiva P;

2. definamos coordenadas $(r, \vec{s})$ como en (5.24), (5.25) y (5.26);

3. definamos $u_{1}(r, \vec{s}):=u(r, \vec{s})=\left[\frac{\delta h_{x}}{\mathbb{H}^{11}-\mathbb{H}^{1,1+a} \gamma_{a}}\right](x(r, \vec{s}), \vec{y}(r, \vec{s}))$;

4. escojamos una constante $\kappa$ cumpliendo (5.32);

5. definamos $v$ como en (5.28).

Observación 5.8. Un punto delicado en el procedimiento descripto en el Teorema 5.3 es la construcción de las coordenadas $G$-adaptadas. Dicha construcción involucra resolver un sistema de ecuaciones diferenciales que puede no ser soluble por cuadraturas. Esa es la razón por la cual usamos el término "solución explícita" para diferenciarlo de las soluciones por cuadraturas presentadas en el capítulo anterior.

\subsection{Ejemplo: péndulo doble invertido}

Como ejemplo del procedimiento desarrollado en las secciones previas, vamos a estudiar el péndulo doble invertido.

Antes de analizar el ejemplo concreto y con el objetivo de facilitar la lectura, presentamos un breve resumen de los resultados desarrollado a lo largo del capítulo para sistemas con dos grados de libertad, con una notación más sencilla. 


\subsubsection{Sistemas con dos grados de libertad}

Supongamos que $\operatorname{dim} Q=2, \operatorname{dim} G=1$ y que $(H, \mathcal{W})$ es un sistema subactuado cinético $G$ invariante. Consideremos que $(x, y)$ son coordenadas $G$-adaptadas y adoptemos para $H$ y $V$ la notación (3.33) y (3.35), i.e.

$$
\mathbb{H}(x, y)=\left[\begin{array}{ll}
a(x, y) & b(x, y) \\
b(x, y) & c(x, y)
\end{array}\right], \quad \mathbb{V}(x, y)=\left[\begin{array}{ll}
f(x, y) & g(x, y) \\
g(x, y) & l(x, y)
\end{array}\right]
$$

Escribamos también

$$
\delta=\frac{f l-g^{2}}{l}, \quad \gamma=\gamma_{1}=\frac{g}{l} .
$$

Con esta notación, el Teorema 5.3 puede reescribirse de la siguiente manera para sistemas con dos grados de libertad

Teorema 5.4. Sea $G$ un grupo de Lie de dimensión 1 que actúa de manera libre y propia sobre una variedad suave $Q$ de dimensión 2. Sea $(H, \mathcal{W})$ un sistema Hamiltoniano subactuado cinético $G$-invariante sobre $Q$ y consideremos un punto de equilibrio inestable $\bar{\alpha} \in T_{\bar{q}}^{*} Q$ de $X_{H}$. Luego, si $(x, y)$ son coordenadas $G$-adaptadas para $(H, \mathcal{W}), y$ verificamos que

$$
h_{x x}(\mathbf{0})>0 \quad \text { obien } \quad\left[b h_{x x}+c h_{x y}\right](\mathbf{0}) \neq 0
$$

podemos construir soluciones locales y definidas positivas de las ecuaciones cinéticas y potenciales del siguiente modo:

1. fijamos una función $\gamma(x)$ que cumpla

$$
a(0)-b(0) \gamma(0) \neq 0, \quad y \quad\left[(a-b \gamma) h_{x x}+(b-c \gamma) h_{x y}\right](\mathbf{0})>0
$$

$y$ definimos

$$
\begin{gathered}
\mathbb{V}^{11}(x):=\delta(x)+\gamma^{2}(x), \quad \mathbb{V}^{12}(x)=\mathbb{V}^{21}(x):=\gamma(x) \\
y \quad \mathbb{V}^{22}(x):=1
\end{gathered}
$$

donde

$$
\delta(x):=P \exp \left(\int_{0}^{x} \frac{B(t)}{a(t)-b(t) \gamma(t)} \mathrm{d} t\right)
$$

para alguna constante positiva $P$;

2. definimos coordenadas $(r, s)$ como sigue

$$
\left\{\begin{array} { l } 
{ r = x + \frac { 1 } { 2 } ( y + \varepsilon ( x ) ) ^ { 2 } } \\
{ s = y + \varepsilon ( x ) }
\end{array} \quad \left\{\begin{array}{l}
x=r-\frac{1}{2} s^{2}, \\
y=s-\varepsilon\left(r-\frac{1}{2} s^{2}\right) .
\end{array} ;\right.\right.
$$

con

$$
\varepsilon(x)=-\int_{0}^{x} \frac{b(t)-\gamma(t) c(t)}{a(t)-b(t) \gamma(t)} \mathrm{d} t
$$


3. definimos

$$
u_{1}(r, s):=u(r, s)=\left[\frac{\delta h_{x}}{a-b \gamma}\right](x(r, s), \vec{y}(r, s))
$$

4. escogemos una constante $\kappa$ tal que

$$
\kappa>P\left[\frac{h_{x y}^{2}}{(a-b \gamma) h_{x x}+(b-c \gamma) h_{x y}}\right](\mathbf{0}) ;
$$

5. definimos $v$ como

$$
v(x, y)=\int_{0}^{\frac{1}{2}(y-\varepsilon(x))^{2}+x} u(t, y-\varepsilon(x)) \mathrm{d} t+\kappa \frac{(y-\varepsilon(x))^{2}}{2}
$$

\subsubsection{El ejemplo}

El espacio de configuraciones para el péndulo doble invertido es el producto $Q=S^{1} \times S^{1}$. Vamos a utilizar coordenadas angulares locales $\varphi_{1}$ y $\varphi_{2}$ medidas respecto de la posición vertical (ver figura). La función Lagrangiana para este sistema está dada por

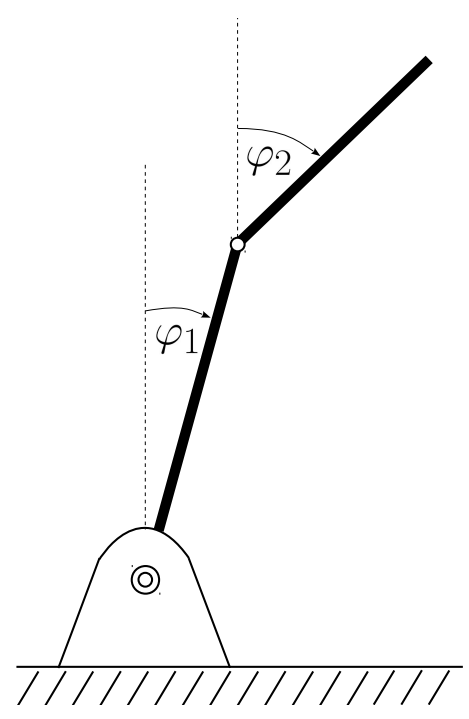

Péndulo doble invertido

$$
\begin{aligned}
L\left(\varphi_{1}, \varphi_{2}, \dot{\varphi}_{1}, \dot{\varphi}_{2}\right) & =\frac{1}{2}\left(\begin{array}{ll}
\dot{\varphi}_{1} & \dot{\varphi}_{2}
\end{array}\right)\left[\begin{array}{cc}
A & B \cos \left(\varphi_{1}-\varphi_{2}\right) \\
B \cos \left(\varphi_{1}-\varphi_{2}\right) & C
\end{array}\right]\left(\begin{array}{c}
\dot{\varphi}_{1} \\
\dot{\varphi}_{2}
\end{array}\right) \\
& -D_{1} \cos \left(\varphi_{1}\right)-D_{2} \cos \left(\varphi_{2}\right),
\end{aligned}
$$


donde $A, B, C, D_{1}$ y $D_{2}$ son constantes positivas relacionadas con las masas y las longitudes de las dos barras. El Hamiltoniano correspondiente, a su vez, está dado por

$$
\begin{aligned}
& H\left(\varphi_{1}, \varphi_{2}, p_{\varphi_{1}}, p_{\varphi_{2}}\right)=\frac{1}{2 \mathbf{M}}\left(\begin{array}{ll}
p_{\varphi_{1}} & p_{\varphi_{2}}
\end{array}\right)\left[\begin{array}{cc}
C & -B \cos \left(\varphi_{1}-\varphi_{2}\right) \\
-B \cos \left(\varphi_{1}-\varphi_{2}\right) & A
\end{array}\right]\left(\begin{array}{l}
p_{\varphi_{1}} \\
p_{\varphi_{2}}
\end{array}\right) \\
& +D_{1} \cos \left(\varphi_{1}\right)+D_{2} \cos \left(\varphi_{2}\right),
\end{aligned}
$$

donde $\mathbf{M}=\mathbf{M}\left(\varphi_{1}, \varphi_{2}\right)=A C-B^{2} \cos ^{2}\left(\varphi_{1}-\varphi_{2}\right)$. Supongamos ahora que queremos estabilizar (asintóticamente) la posición vertical $\left(\varphi_{1}, \varphi_{2}, p_{\varphi_{1}}, p_{\varphi_{2}}\right)=(0,0,0,0)$ con una señal de control con valores en el espacio de actuación $\mathcal{W}$ dado por $W=\operatorname{span}\left\{\mathrm{d} \varphi_{1}\right\}$, i.e. $\xi=\mathrm{d} \varphi_{1}$. Por otro lado, la energía cinética de $H$ y la codistribución $W$ son invariantes ante la acción diagonal inducida por la traslación a izquierda de $S^{1}$ sobre sí mismo, i.e.

$$
\left(\alpha,\left(\varphi_{1}, \varphi_{2}\right)\right) \mapsto\left(\varphi_{1}+\alpha, \varphi_{2}+\alpha\right)
$$

Esta acción resulta ser propia (puesto que $S^{1}$ es un grupo de Lie compacto) y libre y, por lo tanto, tratamos con un sistema cinético $S^{1}$-invariante. Observemos ahora que el generador infinitesimal para esta acción es el campo vectorial $\eta_{Q}=\frac{\partial}{\partial \varphi_{1}}+\frac{\partial}{\partial \varphi_{2}}$, y como $\operatorname{ker}\left(\mathrm{d} \varphi_{1}\right)=\operatorname{span}\left\{\frac{\partial}{\partial \varphi_{2}}\right\}$ tenemos que

$$
\left\langle\xi, \eta_{Q}\right\rangle=\left\langle\mathrm{d} \varphi_{1}, \frac{\partial}{\partial \varphi_{1}}+\frac{\partial}{\partial \varphi_{2}}\right\rangle=1 .
$$

En estas condiciones, podemos proceder como en el Teorema 5.4 para hallar una solución explícita de las ecuaciones cinéticas y potenciales. En primer lugar, tenemos que construir las coordenadas $S^{1}$ adaptadas de la Proposición 5.2 que rectifican los campos vectoriales $\frac{\partial}{\partial \varphi_{2}}$ (que es $S^{1}$-invariante) y $\frac{\partial}{\partial \varphi_{1}}+$ $\frac{\partial}{\partial \varphi_{2}}$. Un cálculo sencillo muestra que $\left\{\mathrm{d} \varphi_{2}-\mathrm{d} \varphi_{1}, \mathrm{~d} \varphi_{1}\right\}$ es la base dual de $\left\{\frac{\partial}{\partial \varphi_{2}}, \frac{\partial}{\partial \varphi_{1}}+\frac{\partial}{\partial \varphi_{2}}\right\}$, de manera que el cambio de coordenadas adecuado resulta ser

$$
\begin{aligned}
& x=\varphi_{2}-\varphi_{1}, \\
& y=\varphi_{1} .
\end{aligned}
$$

Reescribiendo, obtenemos

$$
\begin{aligned}
H\left(x, y, p_{x}, p_{y}\right) & =\frac{1}{2}\left(\begin{array}{ll}
p_{x} & p_{y}
\end{array}\right) \frac{1}{M(x)}\left[\begin{array}{cc}
A+C+2 B \cos (x) & -C-B \cos (x) \\
-C-B \cos (x) & C
\end{array}\right]\left(\begin{array}{l}
p_{x} \\
p_{y}
\end{array}\right) \\
& +D_{1} \cos (y)+D_{2} \cos (x+y),
\end{aligned}
$$

donde $M(x)=A C-B^{2} \cos ^{2}(x)$. Luego, siguiendo la notación de la sección anterior,

$$
a(x)=\frac{A+C+2 B \cos (x)}{M(x)}, \quad b(x)=\frac{-C-B \cos (x)}{M(x)}, \quad c(x)=\frac{C}{M(x)} .
$$


y

$$
h(x, y)=D_{1} \cos (y)+D_{2} \cos (x+y) .
$$

Notemos que, en estas nuevas coordenadas, el estado a estabilizar también se escribe $(0,0,0,0)$, i.e. el cambio de coordenadas deja fijo al origen. Por otra parte

$$
\nabla h(0,0)=\left.\left(-D_{2} \sin (x+y),-D_{1} \sin (y)-D_{2} \sin (x+y)\right)\right|_{(0,0)}=(0,0),
$$

por lo que el origen es un punto crítico de $h$. Por otra parte,

$$
h_{x x}(0,0)=h_{x y}(0,0)=-D_{2}<0,
$$

y

$$
b(0) h_{x x}(0,0)+c(0) h_{x y}(0,0)=\frac{-C-B}{M(0)}\left(-D_{2}\right)+\frac{C}{M(0)}\left(-D_{2}\right)=\frac{B D_{2}}{A C-B^{2}}>0
$$

por lo que estamos en condiciones de seguir los pasos del 1 al 5 del Teorema 5.4. En primer lugar, notemos que

$$
\begin{aligned}
{\left[(a-b \gamma) h_{x x}+(b-c \gamma) h_{x y}\right](\mathbf{0}) } & =\left[a h_{x x}+b h_{x y}-\gamma\left(b h_{x x}+c h_{x y}\right)\right](\mathbf{0}) \\
& =-\frac{A+B}{A C-B^{2}} D_{2}-\gamma(0) \frac{B}{A C-B^{2}} D_{2}
\end{aligned}
$$

Esta expresión es positiva si $\gamma(0)<1-A / B$. Por otro lado, necesitamos que $\gamma(0) \neq a(0) / b(0)=$ $-(A+C+2 B) /(C+B)$ Luego, escojamos una función suave $\gamma$ tal que

$$
\gamma(0)<1-\frac{A}{B} \quad \text { y } \quad \gamma(0) \neq-\frac{A+C+2 B}{C+B}
$$

y definamos

$$
f(x):=\delta(x)+\gamma^{2}(x), \quad g(x):=\gamma(x) \quad \text { y } \quad l(x):=1,
$$

con $\delta(x)$ dada por (5.33) usando las funciones

$$
\begin{aligned}
B(t) & =\frac{1}{\left(A C-B^{2} \cos ^{2}(t)\right)^{2}}\left\{-2 B \sin (t)\left(A C-B^{2} \cos ^{2}(t)\right)-(A+C+2 B \cos (t)) B^{2} \sin (2 t)\right. \\
& \left.-2\left[B \sin (t)\left(A C-B^{2} \cos ^{2}(t)\right)+(C+B \cos (t)) B^{2} \sin (2 t)\right] \gamma(t)-C B^{2} \sin (2 t) \gamma^{2}(t)\right\}
\end{aligned}
$$

y

$$
a(t)-b(t) \gamma(t)=\frac{A+C+2 B \cos (t)+(C+B \cos (t)) \gamma(t)}{A C-B^{2} \cos ^{2}(t)} .
$$

Esto termina el paso 1 del procedimiento.

Para efectuar los pasos restantes, basta escoger $\kappa$ tal que

$$
\kappa>\frac{P D_{2}\left(A C-B^{2}\right)}{B \gamma(0)-A-B}
$$

y definir $v(x, y)$ como en (5.34), donde

$$
\varepsilon(x)=\int_{0}^{x} \frac{A+B \cos (t)+C \gamma(t)}{A+C+2 B \cos (t)+(C+B \cos (t)) \gamma(t)} \mathrm{d} t .
$$


Para terminar el análisis, si queremos utilizar el método LCB simple para estabilizar el péndulo doble invertido en su posición vertical, sólo basta utilizar las funciones construidas arriba para definir la función $\lambda$ como en (1.38). Luego, la señal de control estará dada por

$$
Y\left(x, y, p_{x}, p_{y}\right)=\lambda\left(x, y, p_{x}, p_{y}\right) \frac{\partial}{\partial p_{y}} .
$$





\section{Apéndice}

Aclaración: Adoptaremos la convención de suma sobre índices repetidos. Además, si $\mathbb{A} \in M_{n}(\mathbb{R})$ es una matriz de elementos $\mathbb{A}^{i j}$, su inversa tiene elementos $\mathbb{A}_{i j}$.

Dada una matriz $\mathbb{A} \in M_{n}(\mathbb{R})$ y $1 \leq m<n$, definimos las matrices $\mathbb{A}_{\bar{m}} \in M_{m}(\mathbb{R})$ y $\mathbb{A}_{\underline{m}} \in M_{m}(\mathbb{R})$ cuyos elementos están dados por

$$
\mathbb{A}_{m}^{x y}:=\mathbb{A}^{x y} \quad \forall 1 \leq x, y \leq m,
$$

y

$$
\mathbb{A}_{\underline{m}}^{x y}:=\mathbb{A}^{n-m+x, n-m+y} \quad \forall 1 \leq x, y \leq m .
$$

A su vez, definimos $\mathbb{A}_{\underline{n}}=\mathbb{A}_{\bar{n}}=\mathbb{A}$.

Observación .9. Si $l \leq m$, tenemos que $\left(\mathbb{A}_{\bar{m}}\right)_{\bar{l}}=\mathbb{A}_{\bar{l}}$ y $\left(\mathbb{A}_{\underline{m}}\right)_{\underline{l}}=\mathbb{A}_{\underline{l}}$.

Por otro lado, dados $1 \leq i, j \leq n$ definimos la matriz $\mathbb{A}_{\hat{i j}} \in M_{n-1}(\mathbb{R})$ como

$$
\mathbb{A}_{i j}^{k l}:=\left\{\begin{array}{lll}
\mathbb{A}^{k l} & \text { si } & k<i, l<j \\
\mathbb{A}^{k-1, l} & \text { si } & k>i, l<j \\
\mathbb{A}^{k, l-1} & \text { si } & k<i, l>j \\
\mathbb{A}^{k-1, l-1} & \text { si } & k>i, l>j
\end{array}\right.
$$

Una matriz $\mathbb{A} \in M_{n}(\mathbb{R})$ es definida positiva si y sólo si, para todo $\left(p_{1}, \ldots, p_{n}\right) \in \mathbb{R}^{n}$

$$
\begin{gathered}
p_{i} \mathbb{A}^{i j} p_{j} \geq 0 \quad \text { y } \\
p_{i} \mathbb{A}^{i j} p_{j}=0 \quad \text { sólo si } \quad p_{1}=\cdots=p_{n}=0 .
\end{gathered}
$$

El siguiente criterio es de gran utilidad para verificar si una matriz simétrica es definida positiva

Teorema A.1 (Criterio de Sylvester). Dada $\mathbb{A} \in M_{n}(\mathbb{R})$ simétrica, vale que $\mathbb{A}$ es definida positiva si y sólo si $\operatorname{det}\left(\mathbb{A}_{\bar{m}}\right)>0$ para todo $m=1, \ldots, n$. 
A partir de este criterio podemos demostrar el siguiente criterio alternativo

Teorema A.2 (Criterio de Sylvester). Dada $\mathbb{A} \in M_{n}(\mathbb{R})$ simétrica, vale que $\mathbb{A}$ es definida positiva si y sólo si $\operatorname{det}\left(\mathbb{A}_{\underline{m}}\right)>0$ para todo $m=1, \ldots, n$.

Demostración. Para comenzar, notemos que si $\mathbb{B} \in M_{n}(\mathbb{R})$ es también simétrica y $\mathbb{P} \in O_{n}(\mathbb{R})$ es una matriz ortogonal tal que

$$
\mathbb{B}=\mathbb{P}^{T} \mathbb{A P}
$$

entonces $\mathbb{A}$ es definida positiva si y sólo si $\mathbb{B}$ lo es (la demostración es inmediata utilizando la definición de matriz definida positiva y la ortogonalidad de $\mathbb{P})$. Definamos ahora $\mathbb{P}_{k} \in M_{k}(\mathbb{R})$ como

$$
\mathbb{P}_{k}^{i j}=\left\{\begin{array}{lll}
1 & \text { si } & i+j=k+1 \\
0 & \text { si } & \text { no }
\end{array} .\right.
$$

Es sencillo probar que $\mathbb{P}_{k}$ es simétrica para todo $k$ y que $\mathbb{P}_{k} \in O_{k}(\mathbb{R})$. Luego, en vistas de la observación inicial de la demostración y usando el Teorema A.1, $\mathbb{A}$ es definida positiva si y sólo si $\hat{\mathbb{A}}:=\mathbb{P}_{n} \mathbb{A P}_{n}$ es definida positiva si y sólo si $\operatorname{det}\left(\widehat{\mathbb{A}}_{m}\right)>0$ para todo $m=1, \ldots, n$. Pero

$$
\left(\mathbb{P}_{m} \mathbb{A}_{\underline{m}} \mathbb{P}_{m}\right)^{i j}=\mathbb{P}_{m}^{i l} \mathbb{A}_{\underline{m}}^{l k} \mathbb{P}_{m}^{k j}=\mathbb{A}_{\underline{m}}^{m+1-i, m+1-j}=\mathbb{A}^{n+1-i, n+1-j},
$$

y de forma similar

$$
\hat{\mathbb{A}} \frac{i j}{m}=\left(\mathbb{P}_{n} \mathbb{A P}_{n}\right)^{i j}=\mathbb{P}_{n}^{i l} \mathbb{A}^{l k} \mathbb{P}_{n}^{k j}=\mathbb{A}^{n+1-i, n+1-j},
$$

para todo $1 \leq i, j \leq m$. Luego

$$
\hat{\mathbb{A}}_{\bar{m}}=\mathbb{P}_{m} \mathbb{A}_{\underline{m}} \mathbb{P}_{m},
$$

y por lo tanto

$$
\operatorname{det}\left(\hat{\mathbb{A}}_{m}\right)=\operatorname{det}\left(\mathbb{P}_{m} \mathbb{A}_{\underline{m}} \mathbb{P}_{m}\right)=\operatorname{det}\left(\mathbb{P}_{m}\right) \operatorname{det}\left(\mathbb{A}_{\underline{m}}\right) \operatorname{det}\left(\mathbb{P}_{m}\right)=\operatorname{det}\left(\mathbb{A}_{\underline{m}}\right) .
$$

En conclusión, $\mathbb{A}$ es definida positiva si y sólo si $\operatorname{det}\left(\mathbb{A}_{\underline{m}}\right)>0$ para todo $m=1, \ldots, n$.

Recordemos que el menor $M_{i j}(\mathbb{A})$ de una matriz $\mathbb{A}$ es el determinante de la submatriz que se obtiene de remover la fila $i$ y la columna $j$ de $\mathbb{A}$ y que para todo $1 \leq i, j \leq n$

$$
\sum_{l=1}^{n}(-1)^{i+l} M_{l i}(\mathbb{A}) \mathbb{A}^{l j}=\sum_{l=1}^{n}(-1)^{i+l} M_{i l}(\mathbb{A}) \mathbb{A}^{j l}=\delta_{i}^{j} \operatorname{det}(\mathbb{A}),
$$

donde $\delta_{i}^{j}$ es la delta de Kronecker. Si $\mathbb{A} \in M_{1}(\mathbb{R}) \simeq \mathbb{R}$, definimos $M_{11}(\mathbb{A})=1$.

Observación .10. 1. Notemos que, si $\mathbb{A} \in M_{n}(\mathbb{R})$, entonces $\operatorname{det}\left(\mathbb{A}_{n-1}\right)=M_{11}(\mathbb{A}) \mathrm{y} \operatorname{det}\left(\mathbb{A}_{\overline{n-1}}\right)=$ $M_{n n}(\mathbb{A})$.

2. Si $1 \leq i, j \leq n$, vale que $\operatorname{det}\left(\mathbb{A}_{\hat{i j}}\right)=M_{i j}(\mathbb{A})$. 
De ahora en adelante, dados $m, k \in \mathbb{N}$ y definiendo $n:=m+k$, adoptamos la siguiente convención para los índices utilizados:

$$
\left\{\begin{aligned}
i, j, k, l & =1, \ldots, n \\
\mu, \nu, \sigma, \tau & =1, \ldots, s \\
x, y, z & =1, \ldots, m .
\end{aligned}\right.
$$

Dada $\mathbb{A} \in M_{m+s}(\mathbb{R})$ tal que $\mathbb{A}_{\underline{m}}$ es invertible, definimos $\delta(\mathbb{A}, s) \in M_{s}(\mathbb{R})$

$$
\delta(\mathbb{A}, s)^{\mu \nu}:=\mathbb{A}^{\mu \nu}-\mathbb{A}^{\mu, s+x}\left(\mathbb{A}_{\underline{m}}\right)_{x y} \mathbb{A}^{s+y, \nu} .
$$

El Lema siguiente es de demostración sencilla.

Lema A.1. Dados $m, s \in \mathbb{N} y \mathbb{A} \in M_{m+s}(\mathbb{R})$ tal que $\mathbb{A}_{\underline{m}}$ es invertible, entonces si $r \leq t \leq s$ vale que

$$
\delta(\mathbb{A}, s)_{\underline{r}}=\delta\left(\underline{\mathbb{A}_{m+t}}, t\right)_{\underline{r}} .
$$

Lema A.2. Dados $m, s \in \mathbb{N} y \mathbb{A} \in M_{m+s}(\mathbb{R})$ tal que $\mathbb{A}_{\underline{m}}$ es invertible, entonces para todo $r=1, \ldots, k$ (para simplificar notación escribimos $\left.\delta(\mathbb{A}, s)_{\underline{r}}=\delta_{\underline{r}}\right)$

$$
M_{\mu \nu}\left(\delta_{\underline{r}}\right)=\frac{M_{\mu \nu}\left(\mathbb{A}_{\underline{m+r}}\right)}{\operatorname{det} \mathbb{A}_{\underline{m}}} \quad y \quad \operatorname{det}\left(\delta_{\underline{r}}\right)=\frac{\operatorname{det}\left(\mathbb{A}_{\underline{m+r}}\right)}{\operatorname{det} \mathbb{A}_{\underline{m}}},
$$

para todo $1 \leq \mu, \nu \leq s$

Demostración. Procedamos por inducción en $s$.

Caso $s=1$ : En este caso $r=1$. Denotando $\delta_{\underline{1}}=\delta$ y como [ver Observación .10]

$$
M_{11}(\delta)=1=\frac{\operatorname{det} \mathbb{A}_{\underline{m}}}{\operatorname{det} \mathbb{A}_{\underline{m}}}=\frac{M_{11}\left(\mathbb{A}_{\underline{m+1}}\right)}{\operatorname{det} \mathbb{A}_{\underline{m}}} .
$$

solo debemos probar que

$$
\operatorname{det}(\delta)=\frac{\operatorname{det}(\mathbb{A})}{\operatorname{det} \mathbb{A}_{\underline{m}}}=\frac{\operatorname{det}\left(\mathbb{A}_{\underline{m+1}}\right)}{\operatorname{det} \underline{\mathbb{A}_{\underline{m}}}} .
$$

En primer lugar, notemos que [ver (A.1)]

$$
M_{1,1+x}(\mathbb{A})=\sum_{y=1}^{m}(-1)^{1+y} \mathbb{A}^{1+y, 1} M_{y x}\left(\mathbb{A}_{\underline{m}}\right)
$$

y también

$$
\left(\mathbb{A}_{\underline{m}}\right)_{x y}=\frac{(-1)^{x+y} M_{y x}\left(\mathbb{A}_{\underline{m}}\right)}{\operatorname{det}\left(\mathbb{A}_{\underline{m}}\right)} .
$$


Siguiendo la definición de $\delta$,

$$
\begin{aligned}
\operatorname{det}(\delta) & =\delta=\mathbb{A}^{11}-(-1)^{x+y} \mathbb{A}^{1,1+x} \frac{M_{y x}\left(\mathbb{A}_{\underline{m}}\right)}{\operatorname{det}\left(\mathbb{A}_{\underline{m}}\right)} \mathbb{A}^{1+y, 1} \\
& =\mathbb{A}^{11}-(-1)^{1+x} \frac{\mathbb{A}^{1,1+x}}{\operatorname{det}\left(\mathbb{A}_{\underline{m}}\right)}(-1)^{1+y} \mathbb{A}^{1+y, 1} M_{y x}\left(\mathbb{A}_{\underline{m}}\right) \\
& =\mathbb{A}^{11}-(-1)^{1+x} \frac{\mathbb{A}^{1+x, 1}}{\operatorname{det}\left(\mathbb{A}_{\underline{m}}\right)} M_{1,1+x}(\mathbb{A}) \\
& =\frac{1}{\operatorname{det}\left(\mathbb{A}_{\underline{m}}\right)}\left[\mathbb{A}^{11} \operatorname{det}\left(\mathbb{A}_{\underline{m}}\right)+(-1)^{x} \mathbb{A}^{1+x, 1} M_{1,1+x}(\mathbb{A})\right]
\end{aligned}
$$

Teniendo el cuenta, la Observación .10, obtenemos

$$
\operatorname{det}(\delta)=\frac{1}{\operatorname{det}\left(\mathbb{A}_{\underline{m}}\right)}\left[\mathbb{A}^{11} M_{11}\left(\mathbb{A}_{\underline{m}}\right)+(-1)^{x} \mathbb{A}^{1+x, 1} M_{1,1+x}(\mathbb{A})\right]=\frac{(-1)^{1+i} \mathbb{A}^{1 i} M_{1 i}(\mathbb{A})}{\operatorname{det}\left(\mathbb{A}_{\underline{m}}\right)}=\frac{\operatorname{det}(\mathbb{A})}{\operatorname{det}\left(\mathbb{A}_{\underline{m}}\right)} .
$$

Paso inductivo: Supongamos que la tesis es cierta para $s-1$ y consideremos $\mathbb{A} \in M_{m+s}(\mathbb{R})$. Si $1 \leq r \leq s-1$, usando el Lema A.I

$$
\delta(\mathbb{A}, s)_{\underline{r}}=\delta\left(\mathbb{A}_{\underline{m+s-1}}, s-1\right)_{\underline{r}} .
$$

Luego, utilizando la hipótesis inductiva, para todo $1 \leq \mu, \nu \leq r$

$$
M_{\mu \nu}\left(\delta(\mathbb{A}, s)_{\underline{r}}\right)=M_{\mu \nu}\left(\delta\left(\mathbb{A}_{\underline{m+s-1}}, s-1\right)_{\underline{r}}\right)=\frac{M_{\mu \nu}\left[\left(\mathbb{A}_{\underline{m+s-1}}\right)_{\underline{m+r}}\right]}{\operatorname{det}\left[\left(\mathbb{A}_{\underline{m+s-1}}\right)_{\underline{m}}\right]}=\frac{M_{\mu \nu}\left(\mathbb{A}_{\underline{m+r}}\right)}{\operatorname{det}\left(\mathbb{A}_{\underline{m}}\right)},
$$

y de manera similar

$$
\operatorname{det}\left(\delta(\mathbb{A}, s)_{\underline{r}}\right)=\operatorname{det}\left(\delta\left(\mathbb{A}_{\underline{m+s-1}}, s-1\right)_{\underline{r}}\right)=\frac{\operatorname{det}\left[\left(\mathbb{A}_{\underline{m+s-1}}\right)_{\underline{m+r}}\right]}{\operatorname{det}\left[\left(\mathbb{A}_{\underline{m+s-1}}\right)_{\underline{m}}\right]}=\frac{\operatorname{det}\left(\mathbb{A}_{\underline{m+r}}\right)}{\operatorname{det}\left(\mathbb{A}_{\underline{m}}\right)} .
$$

Sólo resta probar el caso $r=s$, de modo que $\delta(\mathbb{A}, s)_{\underline{r}}=\delta(\mathbb{A}, s)_{\underline{s}}=\delta(\mathbb{A}, s)$ y $\mathbb{A}_{\underline{m+r}}=\mathbb{A}_{\underline{m+s}}=$ $\mathbb{A}$. Notemos primero que $\delta(\mathbb{A}, s)_{\hat{\mu \nu}}=\delta\left(\mathbb{A}_{\hat{\mu \nu}}, s-1\right)$. En efecto, de la Ecuación (A.2) es claro que $\delta^{\mu \nu}$ depende exclusivamente de $\mathbb{A}_{\underline{m}}$ y de la fila $\mu$ y columna $\nu$ de $\mathbb{A}$. Luego, como $\left(\mathbb{A}_{\mu \nu}\right)_{\underline{m}}=\mathbb{A}_{\underline{m}}$, usando la hipótesis inductiva para $\mathbb{A}_{\hat{\mu \nu}}$,

$$
M_{\mu \nu}(\delta(\mathbb{A}, s))=\operatorname{det}\left(\delta(\mathbb{A}, s)_{\hat{\mu \nu}}\right)=\operatorname{det}\left(\delta\left(\mathbb{A}_{\hat{\mu} \nu}, s-1\right)\right)=\frac{\operatorname{det} \mathbb{A}_{\hat{\mu \nu}}}{\operatorname{det}\left[\left(\mathbb{A}_{\hat{\mu \nu}}\right)_{\underline{m}}\right]}=\frac{M_{\mu \nu}(\mathbb{A})}{\operatorname{det} \mathbb{A}_{\underline{m}}},
$$

para todo $1 \leq \mu, \nu \leq s$.

Por último, usando (A.1) para $i=1$ y $j=s+y$

$$
\begin{aligned}
(-1)^{1+i} M_{1 i}(A) A^{s+y, i} & =0 \\
(-1)^{1+\mu} M_{1 \mu}(A) A^{s+y, \mu}+(-1)^{1+s+z} M_{1, s+z}(A) A^{s+y, s+z} & =0 \\
(-1)^{1+\mu} M_{1 \mu}(A) A^{s+y, \mu} & =(-1)^{s+z} M_{1, s+z}(A)\left(A_{\underline{m}}\right)^{y z} .
\end{aligned}
$$


Multiplicando por $\left(\mathbb{A}_{\underline{m}}\right)_{x y}$ y sumando sobre $y=1, \ldots, m$, obtenemos

$$
\begin{aligned}
& (-1)^{1+\mu} M_{1 \mu}(\mathbb{A})\left(\mathbb{A}_{\underline{m}}\right)_{x y} \mathbb{A}^{s+y, \mu}=(-1)^{s+z} M_{1, s+z}(\mathbb{A}) \delta_{x}^{z} \\
& (-1)^{1+\mu} M_{1 \mu}(\mathbb{A})\left(\mathbb{A}_{\underline{m}}\right)_{x y} \mathbb{A}^{s+y, \mu}=(-1)^{s+x} M_{1, s+x}(\mathbb{A}) .
\end{aligned}
$$

Luego, usando nuevamente (A.1)

$$
\begin{aligned}
\operatorname{det}(\delta(\mathbb{A}, s)) & =(-1)^{1+\mu} M_{1 \mu}(\delta(\mathbb{A}, s)) \delta^{1 \mu}=(-1)^{1+\mu} \frac{M_{1 \mu}(\mathbb{A})}{\operatorname{det} \mathbb{A}_{\underline{m}}} \delta^{1 \mu} \\
& =\frac{1}{\operatorname{det} \mathbb{A}_{\underline{m}}}\left[(-1)^{1+\mu} M_{1 \mu}(\mathbb{A}) \mathbb{A}^{1 \mu}-(-1)^{1+\mu} M_{1 \mu}(\mathbb{A}) \mathbb{A}^{1, s+x}\left(\mathbb{A}_{\underline{m}}\right)_{x y} \mathbb{A}^{s+y, \mu}\right],
\end{aligned}
$$

y usando (A.3)

$$
\begin{aligned}
\operatorname{det}(\delta(\mathbb{A}, s)) & =\frac{1}{\operatorname{det} \mathbb{A}_{\underline{m}}}\left[(-1)^{1+\mu} M_{1 \mu}(\mathbb{A}) \mathbb{A}^{1 \mu}-(-1)^{s+x} M_{1, s+x}(\mathbb{A}) \mathbb{A}^{1, s+x}\right] \\
& =\frac{1}{\operatorname{det} \mathbb{A}_{\underline{m}}}\left[(-1)^{1+\mu} M_{1 \mu}(\mathbb{A}) \mathbb{A}^{1 \mu}+(-1)^{1+s+x} M_{1, s+x}(\mathbb{A}) \mathbb{A}^{1, s+x}\right] \\
& =\frac{1}{\operatorname{det} \mathbb{A}_{\underline{m}}}\left[(-1)^{1+i} M_{1 i}(\mathbb{A}) \mathbb{A}^{1 i}\right]=\frac{\operatorname{det} \mathbb{A}}{\operatorname{det} \mathbb{A}_{\underline{m}}} .
\end{aligned}
$$

Corolario A.1. Sean $m, s \in \mathbb{N} y \mathbb{A} \in M_{m+s}(\mathbb{R})$ una matriz simétrica tal que $\operatorname{det} \mathbb{A}_{\underline{m}}$ es definida positiva, entonces $\mathbb{A}$ es definida positiva si y sólo si $\delta(\mathbb{A}, s)$ también lo es.

Demostración. En primer lugar, notemos que $\delta(\mathbb{A}, s)$ es simétrica. Dado que $\mathbb{A}_{\underline{m}}$ es definida positiva, resulta que $\mathbb{A}_{\underline{m}}$ es invertible y además $\operatorname{det} \mathbb{A}_{\underline{r}}=\operatorname{det}\left(\mathbb{A}_{\underline{m}}\right)_{\underline{r}}>0$ para todo $1 \leq r \leq m$ por el Teorema A.2. Por otro lado, según el Lema A.2, si $1 \leq r \leq s$,

$$
\operatorname{det}\left(\delta(\mathbb{A}, s)_{\underline{r}}\right)=\frac{\operatorname{det}\left(\mathbb{A}_{\underline{m+r}}\right)}{\operatorname{det} \mathbb{A}_{\underline{m}}}
$$

y usando el Criterio de Sylvester concluimos que $\mathbb{A}$ es definida positiva si y sólo si $\delta(\mathbb{A}, s)$ lo es. 



\section{Bibliografía}

[1] R. Abraham and J.E. Marsden, Foundation of Mechanics, Benjaming Cummings, New York, 1985.

[2] D. R. Auckly, L. V. Kapitanski and W. White, Control of nonlinear underactuated systems, Comm. Pure Appl. Math., 53 (3) (2000), 354-369.

[3] A. Bacciotti, L. Rosier, Regularity of Liapunov functions for stable systems, Systems \& Control Letters 41 (2000), 265-270.

[4] A. M. Bloch, P. S. Krishnaprasad, J.E. Marsden and G. Sánchez de Alvarez, Stabilization of Rigid Body Dynamics by Internal and External Torques, Automatica, 28, no. 4 (1992), 745-756.

[5] A. M. Bloch, N.E. Leonard and J.E. Marsden, Stabilization of Mechanical Systems Using Controlled Lagrangians, Proc. of the 36th IEEE Conf. on Decision and Control, 1997, 2356-2361.

[6] A.M. Bloch, N.E. Leonard and J.E. Marsden, Controlled Lagrangian and the stabilization of mechanical systems I: The first matching theorem, IEEE Trans. Automat. Control, 45 (2000), 2253-2270.

[7] A.M. Bloch, D.E. Chang, N.E. Leonard and J.E. Marsden, Controlled Lagrangian and the stabilization of mechanical systems II: Potential shaping, IEEE Trans. Automat. Control, 46 (2001), 1556-1571.

[8] W.M. Boothby, An Introduction to Differentiable Manifolds and Riemannian Geometry, $2^{\text {nd }}$ edition, Academic Press, New York, 2002.

[9] F. Bullo and A. Lewis, Geometric Control of Mechanical Systems, Springer-Verlag, New York, 2005.

[10] M. Chaalal and N. Achour, Stabilization of a Class of Mechanical Systems with Impulse Effects by Lyapunov Constraints, 20th International Conference on Methods and Models in Automation and Robotics (MMAR), 2015, 335 - 340.

[11] D. Chang, A. M. Bloch, N. E. Leonard, J. E. Marsden and C. Woolsey. The equivalence of controlled Lagrangian and controlled Hamiltonian systems, ESAIM: Control, Optimisation and Calculus of Variations, (2001). 
[12] D. E. Chang, The Method of Controlled Lagrangians: Energy Plus Force Shaping, SIAM J. Control and Optimization, 48 no. 8 (2010), 4821-4845.

[13] D.E. Chang, Stabilizability of Controlled Lagrangian Systems of Two Degrees of Freedom and One Degree of Under-Actuation, IEEE Trans. Automat. Contr., 55 no. 8 (2010), 1888-1893.

[14] D.E. Chang, Generalization of the IDA-PBC Method for Stabilization of Mechanical Systems, Proc. of the 18th Mediterranean Conf. on Control \& Automation, 2010, 226-230.

[15] D.E. Chang, On the Method of Interconnection and Damping Assignment Passivity-Based Control for the Stabilization of Mechanical Systems, Regular and Chaotic Dynamics, 19 no. 5 (2014), 556-575.

[16] N. Crasta, R. Ortega, H. Pillai, J. Velazquez, The matching equations of energy shaping controllers for mechanical systems are not simplified with generalized forces, Proceedings of the 4th IFAC Workshop on Lagrangian and Hamiltonian Methods for Non Linear Control, University of Bologna, Bertinoro, Italy (2012).

[17] H.L. Goldschmidt, Existence theorems for analytic linear partial differential equations, Annals of Mathematics, Second Series 86 (1967), 246-270.

[18] S. Grillo, Sistemas noholónomos generalizados, Ph.D. thesis, Instituto Balseiro (2007).

[19] S.D. Grillo, Higher order constrained Hamiltonian systems, Journal of Mathematical Physics, 50 (2009).

[20] S.D. Grillo, F. Maciel and D. Perez, Closed-loop and constrained mechanical systems, Int. Journal of Geom. Meth. in Mod. Physics, 7 (2010).

[21] S. Grillo, F. Maciel and D. Perez, Closed-loop and constrained mechanical systems, Int. Journal of Geom. Meth. in Mod. Physics, 7 (2010).

[22] S.D. Grillo, J. Marsden and S. Nair, Lyapunov constraints and global asymptotic stabilization, Journal of Geom. Mech., 3 (2011), 145-196.

[23] S. D. Grillo, L. M. Salomone and M. Zuccalli, On the relationship between the energy shaping and the Lyapunov constraint based methods, to be published.

[24] S. D. Grillo, L. M. Salomone and M. Zuccalli, Asymptotic stabilizability of underactuated systems with 2 degrees of freedom, to be published.

[25] J. Hamberg, Gerneral matching conditions in the theory of controlled Lagrangians, in Proc. CDC, Phoenix, AZ, 1999.

[26] C. Kellett, Classical converse theorems in Lyapunov's second method, arXiv:1502.04809v2 [math.OC]. 
[27] H.K. Khalil, Nonlinear systems, $3^{\text {rd }}$ edition, Prentice Hall, New Jersey, 2002.

[28] P. Krishnaprasad, Lie-Poisson structures, dual-spin spacecraft and asymptotic stability, Nonl. Anal. Th. Meth. and Appl., 9 (1985), 1011-1035.

[29] J.M. Lee, Introduction to Smooth Manifolds, $2^{\text {nd }}$ edition, Springer-Verlag, New York, 2013.

[30] A.D. Lewis, Potential energy shaping after kinetic energy shaping, in $45^{\text {th }}$ IEEE Conference on Decision and Control, (2006).

[31] J.L. Massera, Contributions to stability theory, Annals of Math 64 (1956), 182-206. Erratum in Annals of Math 68 (1958), 202.

[32] C.-M. Marle, Kinematic and geometric constraints, servomechanism and control of mechanical systems, Rend. Sem. Mat. Univ. Pol. Torino, 54 (1996), 353-364.

[33] C.-M. Marle, Various approaches to conservative and nonconservative non-holonomic systems, Rep. Math. Phys., 42 (1998), 211-229.

[34] J.E. Marsden and T.S. Ratiu, Introduction to Mechanics and Symmetry, $2^{\text {nd }}$ edition, Springer-Verlag, New York, 1999.

[35] R. Ortega, M. W. Spong, F. Gómez-Estern and G. Blankenstein. Stabilization of underactuated mechanical systems via interconnection and damping assignment. IEEE Trans. Aut. Control, 47 (2002), 1281-1233.

[36] J.G. Romero, A. Donaire, and R. Ortega, Robust Energy Shaping Control of Mechanical Systems, Syst. Control Lett., 62 no. 9 (2013), 770-780.

[37] A.S. Shiriaev, J.W. Perram and C.C. Canudas, Constructive tool for orbital stabilization of underactuated nonlinear systems: virtual constraints approach, IEEE Trans. Automat. Contr., 50 (2005), 1164-1176.

[38] A.J. van der Schaft, Stabilization of Hamiltonian systems, Nonlinear Analysis, Theory, Methods \& Applications, 10 (1986), 1021-1035.

[39] C. Woolsey, C. Reddy, A. Bloch, D. Chang, N. Leonard, J. Marsden, Controlled Lagrangian systems with gyroscopic forcing and dissipation, European Journal of Control, 10 (5) (2004), 1-27. 